DESY 02-004

hep-ph/0201075

\title{
Results and Techniques of Multi-Loop Calculations
}

\author{
Matthias Steinhauser \\ II. Institut für Theoretische Physik, \\ Universität Hamburg, D-22761 Hamburg, Germany
}

\begin{abstract}
In this review some recent multi-loop results obtained in the framework of perturbative Quantum Chromodynamics (QCD) and Quantum Electrodynamics (QED) are discussed. After reviewing the most advanced techniques used for the computation of renormalization group functions, we consider the decoupling of heavy quarks. In particular, an effective method for the evaluation of the decoupling constants is presented and explicit results are given. Furthermore the connection to observables involving a scalar Higgs boson is worked out in detail. An all-order low energy theorem is derived which establishes a relation between the coefficient functions in the hadronic Higgs decay and the decoupling constants. We review the radiative corrections of a Higgs boson into gluons and quarks and present explicit results up to order $\alpha_{s}^{4}$ and $\alpha_{s}^{3}$, respectively. In this review special emphasis is put on the applications of asymptotic expansions. A method is described which combines expansion terms of different kinematical regions with the help of conformal mapping and Padé approximation. This method allows us to proceed beyond the present scope of exact multi-loop calculations. As far as physical processes are concerned, we review the computation of three-loop current correlators in QCD taking into account the full mass-dependence. In particular, we concentrate on the evaluation of the total cross section for the production of hadrons in $e^{+} e^{-}$annihilation. The knowledge of the complete mass dependence at order $\alpha_{s}^{2}$ has triggered a bunch of theory-driven analyses of the hadronic contribution to the electromagnetic coupling evaluated at high energy scales. The status is summarized in this review. In a further application four-loop diagrams are considered which contribute to the order $\alpha^{2}$ QED corrections to the $\mu$ decay. Its relevance for the determination of the Fermi constant $G_{F}$ is discussed. Finally the calculation of the three-loop relation between the $\overline{\mathrm{MS}}$ and on-shell quark mass definitions is presented and physical applications are given. To complete the presentation, some technical details are presented in the Appendix, where also explicit analytical results are listed.
\end{abstract}

(To appear in Physics Reports) 


\section{Contents}

\begin{tabular}{llr}
\hline 1 & Introduction & 2
\end{tabular}

\begin{tabular}{|lll}
\hline 2 & Renormalization group functions in QCD & 6
\end{tabular}

2.1 Ultra-violet and infra-red counterterms . . . . . . . . . . . . . . . . 6

2.2 Global infra-red re-arrangement and the quark anomalous dimension . . . 11

2.3 Massive vacuum integrals and the $\beta$ function . . . . . . . . . . . . . . . . . 18

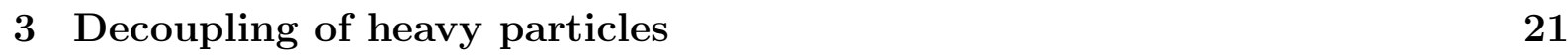

3.1 Construction of an effective Lagrangian in QCD . . . . . . . . . . . . . . . 23

3.2 Computation of the decoupling constants . . . . . . . . . . . . . . . . . 24

3.3 Results . . . . . . . . . . . . . . . . . . . . . . . . . . 29

3.4 Application . . . . . . . . . . . . . . . . . . . . . 31

4 Scalar dimension-four operators in QCD 34

4.1 Hadronic Higgs decay . . . . . . . . . . . . . . . . . . . . . . . . . . . . . . 38

4.1 .1 Direct calculation of the coefficient functions . . . . . . . . . . . . . 42

4.1 .2 Low-energy theorem . . . . . . . . . . . . . . . . . . . . 43

$4.1 .3 \quad H \rightarrow g g$ in the background field method . . . . . . . . . . . . . . . 47

4.1 .4 Hadronic decay rate of the SM Higgs boson . . . . . . . . . . . . . 50

4.2 Quartic mass corrections to $\sigma\left(e^{+} e^{-} \rightarrow\right.$ hadrons $)$. . . . . . . . . . . . . . . 56

5 Asymptotic expansion and Padé approximation 63

5.1 The Method . . . . . . . . . . . . . . . . . . . . . 65

5.1 .1 Explicit example at two loops . . . . . . . . . . . . . . . . . 70

5.2 Current correlators in QCD . . . . . . . . . . . . . . . . . . 71

5.2 .1 Diagonal current correlators . . . . . . . . . . . . . . . . . . . 73

5.2 .2 An application of $R(s): \Delta \alpha_{\text {had }}\left(M_{Z}\right)$. . . . . . . . . . . . . . . . . 81

5.2 .3 Heavy-light current correlators . . . . . . . . . . . . . . . . . . . . . 85

5.3 QED corrections to muon decay . . . . . . . . . . . . . . . . . . . . . . . . 89

5.4 The relation between the MS and on-shell quark mass . . . . . . . . . . . . 95

\begin{tabular}{|ll}
\hline A Technical remarks & 101
\end{tabular}

A.1 Asymptotic expansion . . . . . . . . . . . . . . . . . . . . . . . . . . . 101

A.2 Single-scale Feynman diagrams up to three loops . . . . . . . . . . . . . . . 104

A.3 Automation of Feynman diagram computation . . . . . . . . . . . . . . . . 108

B Decoupling constants and coefficient functions 109

\begin{tabular}{ll|}
\hline C Analytical results for $R(s)$ & 114
\end{tabular} 


\section{Introduction}

Nowadays the Standard Model (SM) of elementary particle physics is well established. Some parts of it (e.g. the properties of the $Z$ boson) have been tested to an accuracy far below the percent level - mostly at the CERN Large-Electron-Positron collider (LEP, Geneva), at the SLAC Linear Collider (SLC, Stanford) and at the Fermilab TEVATRON (Chicago). Up to now no significant deviation between theory and experiment has been found. For other parts of the SM, related to $\mathrm{CP}$ violation and quark mixing, the $B$ factories like BaBar at SLAC, Belle at KEK (Tsukuba) or HERA-B at DESY (Hamburg) will provide deeper insight, and significant improvements in the determination of the corresponding parameters will be obtained. Currently mainly the scalar sector of the SM eludes from direct experimental observation. This affects both the generation of the particle masses and the existence of the Higgs boson itself. Probably Run II of the TEVATRON and certainly the Large Hadron Collider (LHC) at CERN will provide more sureness - not only in connection to the Higgs sector but also to possible extensions of the SM. Once the Higgs boson would be discovered it immediately would become subject to precision measurements. In particular at a future $e^{+} e^{-}$linear collider such as DESY TESLA, a precise study of its properties would be possible.

In the recent years there has been an enormous development in the evaluation of radiative corrections. It is fair to say that the major part of it was initiated by the fundamental works of 't Hooft and Veltman in 1972 [1, 2, 3] where dimensional regularization (see also [4]) was established as a powerful tool in the evaluation of multi-loop diagrams $\$$. Since that time a whole industry has been formed to develop techniques for the computation of complicated Feynman integrals. At one-loop order the procedure of the computation has been systematically studied quite some time ago [6, 7, 8, 9]. Nevertheless also nowadays it is not completely straightforward to evaluate an arbitrary one-loop diagram - in particular if many legs and complicated momentum configurations are involved. One can easily imagine that at two and more loops one arrives quite soon at the limit where the occuring mathematical expressions can not be solved. At two-loop order certain classes of diagrams can still be treated by either using a combination of analytical simplifications and fast numerical routines, like in the case of two-point function with several non-zero masses [10], or applying purely analytical methods, like in the case of massless digrams with four external legs [11]. However, at three-loop order it is essentially only possible to solve one-scale integrals. A systematic study at four or more loops is still missing.

QCD, the field theoretical realization of the strong interaction, constitutes an important part of the SM and also of most of its extensions. At low energies the coupling constant of $\mathrm{QCD}, \alpha_{s}$, is large and perturbative calculations are not possible. However, due to the phenomenon of asymptotic freedom the value of $\alpha_{s}$ gets smaller with raising energy and perturbation theory is an appropriate tool to evaluate radiative corrections.

Up to now the vast majority of the multi-loop calculations have been performed in the

\footnotetext{
${ }^{1}$ Dimensional regularization applied to infra-red divergences and mass singularities has first been considered in $[5$.
} 
framework of QED and QCD. One reason is certainly that the calculations are simpler as compared to the full SM since there are less parameters. Furthermore, there is a strong hierarchy both in the quark and lepton masses which also simplifies the calculations. On the other hand, the higher-order corrections are indeed necessary. In QED there exist precise experiments which require high theoretical precision and although the coupling constant is quite small sometimes high loop orders are necessary. For example, in the case of the anomalous magnetic moment of the electron, four-loop corrections are needed to match the experimental precision. In QCD the coupling is roughly a factor of ten bigger. Nevertheless it is often still small enough to perform a perturbative expansion. However, the higher order terms are significant and can not be neglected in the cases where high precision is required.

In this work some recent developments in the calculation of multi-loop diagrams are reviewed. Thereby we will mention the most important methods which have been used in the computation of higher order quantum corrections and explain a few selected ones in greater detail. At the same time, we discuss the present theoretical status of important physical quantities. In particular, the renormalization group functions in the modified minimal subtraction scheme [12, 13] $(\overline{\mathrm{MS}})$ are provided up to the four-loop order. As is well-known, four loop running must be accompanied by three-loop matching at quark thresholds. The corresponding decoupling relations are presented in Section 2 .

The hadronic Higgs decay is closely connected to the decoupling relations as we will show is Section 1 . Parts of the quantum corrections can be computed in the framework of an effective Lagrangian where the coefficients can be determined from the decoupling relations. The origin of this miraculous connection lies in the use of the dimension-four operators, which constitute an important ingredient of the effective Lagrangian. Another application of the dimension-four operators are the quartic mass corrections to the cross section $\sigma\left(e^{+} e^{-} \rightarrow\right.$ hadrons $)$, which is also discussed in Section 1 .

The last issue is again picked up in Section 5, where also QCD corrections to the production of hadrons in $e^{+} e^{-}$annihilation are computed. Putting together all terms one arrives at a complete picture up to the quartic mass corrections of order $\alpha_{s}^{3}$. The main purposes of Section 5 are practical applications of asymptotic expansions? Besides the diagonal correlators also the non-diagonal ones are considered.

The Fermi constant, $G_{F}$, the mass of the $Z$ boson, $M_{Z}$, and the electromagnetic coupling constant, $\alpha$, are the best known parameters of the SM. $M_{Z}$ has been measured at LEP with an accuracy of a few per mille to be $M_{Z}=91.1876 \pm 0.0021 \mathrm{GeV}$. In this review we want to discuss quantum corrections to the other two parameters. An essential ingredient to the running of the electromagnetic coupling from $q^{2}=0$ to $q^{2}=M_{Z}^{2}$ is provided by the cross section $\sigma\left(e^{+} e^{-} \rightarrow\right.$ hadrons). The correction terms discussed earlier have been used to obtain so-called theory-driven results. The different approaches are discussed. As further applications we present the status of the QED corrections to the muon decay and the relation between the $\overline{\mathrm{MS}}$ and the on-shell quark mass. Let us in the following discuss the individual issues in more detail.

\footnotetext{
${ }^{2}$ See Appendix A.1 for details.
} 
As far as radiative corrections are concerned, a crucial role is played by the renormalization group functions. In particular the functions $\beta\left(\alpha_{s}\right)$ and $\gamma_{m}\left(\alpha_{s}\right)$ governing the running of the coupling and the quark masses comprise a significant part of the higher quantum corrections. They are in particular very important to re-sum large logarithms to all orders in perturbation theory. Only a few years ago the four-loop terms of order $\alpha_{s}^{4}$ have been evaluated for $\beta\left(\alpha_{s}\right)$ [14 and $\gamma_{m}\left(\alpha_{s}\right)$ [15, 16]. The latter has been computed by two groups using completely independent methods. Both methods are based on the fact that the pole part of a logarithmically divergent diagram is independent of the masses or momenta. In [15] this is exploited together with the technique of infra-red re-arrangement (IRR) in order to obtain a factorization of the four-loop integrals into massless three-loop and massive one-loop ones. In Refs. [14, 16] all lines were assigned to the same mass, and all external momenta were set to zero, which leads to a special class of bubble diagrams. From them only the pole parts have to be computed. In Section 2 we want to review both methods and explicitly demonstrate the way they work.

In this review special emphasis is put on the construction of effective theories in the framework of QCD. In Section 3 an effective QCD Lagrangian is constructed for the case where one of the quarks is much heavier than the others. The construction is made explicit by specifying the relations between the parameters in the full and effective theories. These relations provide at the same time the well-known decoupling constants which have to be applied in QCD every time a particle threshold is crossed. The most prominent example for their necessity is probably the computation of $\alpha_{s}\left(M_{\tau}\right)$ from $\alpha_{s}\left(M_{Z}\right)$ or vice versa. In the latter case five quarks are active whereas in the former one only three quarks are present in the effective QCD Lagrangian.

In Section 4 a slightly different point of view is adopted. Here the scalar operators of dimension four are considered in QCD. In a first step they are used to construct an effective Lagrangian describing the coupling of an intermediate-mass Higgs boson to quarks and gluons. The top quark is considered as heavy and manifests itself in the coefficient functions of the effective Lagrangian. Once the latter has been found, the imaginary part of the Higgs boson correlator in the effective theory leads to the total decay rate. As a central result of Section 4 we derive a low-energy theorem which considerably simplifies the computation of the coefficient functions as they are related to the decoupling constants of QCD evaluated in Section 3. In Section 4.1.3 the background field method is introduced as a convenient tool for the computation of higher-order corrections. As an example, the coefficient functions describing the decay of the Higgs boson into gluons is also computed in this framework.

In the second part of Section 1 another important application of the scalar dimension four operators is discussed, namely the quartic mass corrections to the cross section $\sigma\left(e^{+} e^{-} \rightarrow\right.$ hadrons $)$. Mass corrections of order $\left(\mathrm{m}^{2} / \mathrm{s}\right)^{0}$ and $\left(\mathrm{m}^{2} / \mathrm{s}\right)^{1}$ are obtained relatively easy as in QCD there are no non-trivial operators of dimension less than four. However, the quartic corrections require the inclusion of the dimension-four operators with all their renormalization and mixing properties. We will explain the techniques and present results obtained recently at order $\alpha_{s}^{3}$.

The last part of this review, Section 5, is devoted to the discussion of some results 
obtained with the help of asymptotic expansion accompanied with conformal mapping and Padé approximation. This method has been developed in the recent years and has been applied successfully to a number of important processes. The underlying idea is the following: only in rare cases it is possible to compute three-loop diagrams involving more than one scale. However, if a certain hierarchy exists between the scales it is promising to apply an asymptotic expansion. This effectively reduces the number of scales present in the integrals which are subsequently significantly simplified.

In particular we will discuss the corrections of order $\alpha_{s}^{2}$ to the photon polarization function. Its imaginary part is directly connected to the physical quantity $R(s) \equiv \sigma\left(e^{+} e^{-} \rightarrow\right.$ hadrons $) / \sigma\left(e^{+} e^{-} \rightarrow \mu^{+} \mu^{-}\right)$. The application of our method leads to the full mass dependence. Combining the results with the quartic corrections given in Section 4.2, one obtains a prediction for $R(s)$ up to and including $\mathcal{O}\left(\alpha_{s}^{3} \mathrm{~m}^{4} / \mathrm{s}^{2}\right)$. Only recently also the non-diagonal current correlator formed by a massive and a massless quark has been computed. In this application special emphasis lies on the extraction of information about the threshold behaviour which has some relevance in the framework of heavy-quark effective QCD.

As an application of the knowledge of $R(s)$ to high perturbative order, we discuss the evaluation of $\alpha\left(M_{Z}^{2}\right)$. The electromagnetic coupling is defined at vanishing momentum transfer. However, its evolution to high energies constitutes the dominant part of the radiative corrections to electroweak observables. The accurate determination of $\alpha\left(M_{Z}^{2}\right)$ is thus essential for any precise test of the theory. At the same time the indirect determination of the masses of heavy, hitherto unobserved particles, e.g. the Higgs boson or supersymmertic particles, depends critically on this parameter. Of particular importance in this context is the hadronic vacuum polarization. It is nearly as large as the leptonic contribution, but cannot yet be computed perturbatively. However, it may be related through dispersion relations to the cross section for hadron production in electronpositron annihilation. The integrand can thus be obtained from data, phenomenological models and/or perturbative QCD, whenever applicable. In Section 5.2.2 we will discuss the developments in the evaluation of $\alpha\left(M_{Z}^{2}\right)$ which took place in the recent two to three years due to the knowledge of the complete mass dependence of $R(s)$ at order $\alpha_{s}^{2}$ (cf. Section 5.2.1).

$G_{F}$ is defined through the muon lifetime, and the decay of the muon, as a purely leptonic process, is rather clean - both experimentally and theoretically. The one-loop corrections of order $\alpha$ were computed more than 40 years ago [17], whereas only recently the two-loop corrections of order $\alpha^{2}$ have been evaluated [18, 19]. The large gap in time shows that this calculation is highly non-trivial. The inclusion of the two-loop terms greatly reduced the relative theoretical error of $1.5 \times 10^{-5}$ which was an estimate of the size of the missing corrections. The remaining error on $G_{F}$ now reads $0.9 \times 10^{-5}$ and is of pure experimental nature. Upcoming experiments will further improve the accuracy of the muon lifetime measurement and therefore the $\mathcal{O}\left(\alpha^{2}\right)$ corrections to the muon decay are very important and constitute a crucial ingredient from the theoretical side. In Section 5.3 we discuss the results obtained with the help of asymptotic expansion.

In the SM the quark masses have still relatively big uncertainties. This is mainly due 
to the confinement property of QCD which prevents the production of free quarks. It is also important to have a convenient definition of the quark mass in order to perform a comparison between theory and experiment. Recently there has been quite some activity connected to the precise determination of the bottom- and top-quark masses. The bottomquark mass is determined with the help of QCD sum rules where a proper mass definition helps to reduce the error. In the case of the top quark, studies have been performed for an $e^{+} e^{-}$collider with a center-of-mass energy in the threshold region of top-quark-pair production. An energy scan which provides the measurement of the total production cross section would provide an error of about $100 \mathrm{MeV}$ in the top-quark mass. Also here a special mass definition has to be employed. In both cases the three-loop on-shell- $\overline{\mathrm{MS}}$ conversion formula is needed in order to obtain the corresponding $\overline{\mathrm{MS}}$ quark mass. The latter is important for processes not connected to the threshold. In Section 5.4 these issues are discussed in detail.

\section{Renormalization group functions in QCD}

In perturbative QCD the renormalization group functions play a very important role. In particular the $\beta$ and $\gamma_{m}$ functions governing the running of the strong coupling and the quark masses, respectively, are indispensable when evaluating physical observables. For this reason we decided to discuss the techniques used for the computation of the four-loop contributions to $\beta$ [14] and $\gamma_{m}$ [15, 16] in more detail.

In the $\overline{\mathrm{MS}}$ scheme the knowledge of the renormalization group functions is equivalent to the knowledge of the corresponding renormalization constant. Thus, in order to compute a renormalization constant at $n$-loop order it is sufficient to evaluate the ultra-violet (UV) poles of $n$-loop diagrams. Nevertheless it is often also quite useful to have also a handle on the infra-red (IR) poles. More details on the UV and IR structure of Feynman diagrams are given in Section 2.1. Afterwards, in Sections 2.2 and 2.3, two practical methods are described which have been applied at the four-loop level.

\subsection{Ultra-violet and infra-red counterterms}

Before we want to consider explicit examples in the next two sections the theoretical background needed for the higher-loop calculation of renormalization group functions is introduced in this Subsection. In particular we want to demonstrate the interplay between UV and IR divergences in dimensional regularization and show their connection to asymptotic expansions. We refrain from presenting the material in a mathematical rigorous framework, for which we refer to the original literature, but exemplify the important points at explicit diagrams.

As the properties discussed in this section are independent of the particle type we consider only scalar propagators of the form

$$
\frac{1}{M^{2}-p^{2}}
$$


where also $M=0$ is allowed. We furthermore assume that the integration momenta are denoted by $k_{1}, k_{2}, \ldots$ and introduce the abbreviation $\int_{i} \equiv \mu^{4-D} \int \mathrm{d}^{D} k_{i} /(2 \pi)^{D}$.

In order to remove the UV divergences the so-called $R$ operation has been introduced [20, 21]. It is a recursive subtraction scheme where the UV divergences are removed from the Feynman integrals in a way compatible to adding local counterterms to the Lagrangian. Formally, the $R$ operation can be written as a sum where each term is a product of operators acting on subsets of disjoint one-particle-irreducible subgraphs. In this way counterterms are generated. They have to be inserted into the vertices of the diagrams which remain after shrinking the corresponding subgraphs to a point. Non-trivial diagrammatic examples can, e.g., be found in the text books [22, 23]. The $R$ operation applied to a one-loop propagator-type integral with external momentum $q$ leads to the equation

$$
\begin{aligned}
R\left[\int_{1} \frac{1}{\left[M^{2}-k_{1}^{2}\right]\left[M^{2}-\left(k_{1}+q\right)^{2}\right]}\right] & =\int_{1} \frac{1}{\left[M^{2}-k_{1}^{2}\right]\left[M^{2}-\left(k_{1}+q\right)^{2}\right]}+Z^{(1)} \\
& =\text { finite, }
\end{aligned}
$$

from which the renormalization constant $Z^{(1)}$ is determined. In the $\overline{\mathrm{MS}}$ scheme it reads

$$
Z^{(1)}=\frac{1}{16 \pi^{2}}\left(-\frac{1}{\varepsilon}\right)
$$

Once $Z^{(1)}$ is known one can turn to the two-loop order. The application of the $R$ operation to the diagram shown in Fig. 2.1 leads to

$$
\begin{aligned}
R & {\left[\int_{1} \int_{2} \frac{1}{\left[M^{2}-\left(k_{1}-k_{2}\right)^{2}\right]\left[M^{2}-\left(k_{1}+q\right)^{2}\right]\left[m^{2}-k_{2}^{2}\right]^{2}}\right] } \\
& =\int_{1} \int_{2} \frac{1}{\left[M^{2}-\left(k_{1}-k_{2}\right)^{2}\right]\left[M^{2}-\left(k_{1}+q\right)^{2}\right]\left[m^{2}-k_{2}^{2}\right]^{2}}+Z^{(1)} \int_{2} \frac{1}{\left[m^{2}-k_{2}^{2}\right]^{2}}+Z^{(2)} \\
& =\text { finite, }
\end{aligned}
$$

which fixes $Z^{(2)}$ to

$$
Z^{(2)}=\left(\frac{1}{16 \pi^{2}}\right)^{2}\left[\frac{1}{2 \varepsilon^{2}}-\frac{1}{2 \varepsilon}\right]
$$

A formal definition of the $R$ operation can, e.g., be found in 24.

There are two special features of dimensional regularization which prove to be a powerful tool, especially in the evaluation of renormalization group functions. The first one is that dimensional regularization is able to regularize simultaneously both UV and IR divergences. Furthermore, as was realized in [25], in renormalization schemes based on minimal subtraction all UV counterterms are polynomial in the momenta[ and masses. This means that the divergence of logarithmically divergent Feynman integrals are polynomial in $1 / \varepsilon$ and there is no dependence on a dimensionful scale. As a consequence, in the computation

\footnotetext{
${ }^{3}$ This has to be the case for each meaningful renormalization prescription.
} 


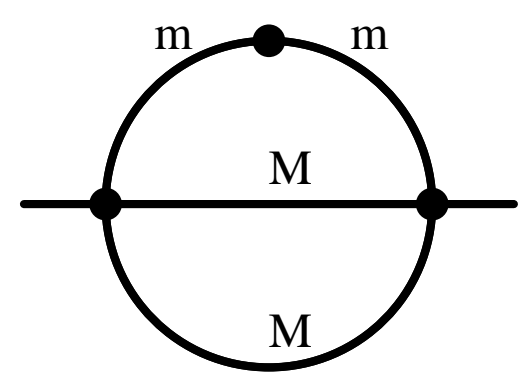

Figure 2.1: Scalar two-loop diagram.

of the corresponding coefficients one is free to make arbitrary re-arrangements of masses and external momenta - provided no IR divergences are introduced. This was for the first time observed in Ref. [26] (see also [27, 28, 24]).

In the example of Eqs. (2.2) and (2.4) this means that the masses $M$ could be set to zero without changing the results for $Z^{(1)}$ and $Z^{(2)}$.

This procedure is often referred to as infra-red re-arrangement (IRR) [27]. One has in mind a transformation of the IR structure of a diagram in such a way that its UV divergent part can be easily computed. Next to nullifying masses or momenta, also differentiations with respect to masses are allowed in order to achieve the simplifications.

The requirement that no IR divergences may be introduced is quite restrictive and makes the application of the IRR very tedious. Often one has to remain with Feynman integrals which are not as simple as one would like to have them. To overcome this disadvantage the $R$ operation has been generalized and the so-called $R^{\star}$ operations has been developed [29, 30] in order to deal not only with the UV but also with the IR divergences. Thus arbitrary re-arrangements of masses and momenta are allowed as the $R^{\star}$ operation takes care of all occuring IR divergences.

The $R^{\star}$ operation can be written as $R^{\star}=R \tilde{R}=\tilde{R} R$, where $R$ is responsible for the UV divergences and $\tilde{R}$ subtracts the IR ones. For $\tilde{R}$ there exists a recursive definition which is in close analogy to the one of the $R$ operation. The precise definition and the criterion for the subgraphs, which have to be considered while applying $\tilde{R}$, is quite involved and requires the introduction of a lot of mathematical terminology [30]. The way $\tilde{Z}-$ the renormalization constant generated by $\tilde{R}$ - is computed in practice can best be seen by looking at the examples discussed below. At this point we only want to mention that a renormalization constant $\tilde{Z}$ for the whole diagram only appears for scaleless integrals. As a consequence, the $\tilde{R}$ operation does not commute with the limit of masses or external momenta going to zero (cf. Eqs. (2.8) and (2.9) below).

The $R^{\star}$ operation is essential to prove the following theorem 30]:

Any $U V$ counterterm of a $(l+1)$-loop Feynman integral can be written in terms of the poles and finite parts of appropriately constructed l-loop massless propagator-type integrals.

This theorem is very powerful. It states that — at least in principle - all renormalization 
group functions at four-loop order can be computed from the knowledge of massless threeloop two-point functions. The latter are, e.g., provided by the package MINCER [31]. In practice there are problems connected to the (in general) large number of contributing diagrams as the prescriptions for the $R^{\star}$ operation given in [29, 30] have to be applied individually to each of them. Thus, for practical applications further improvements are necessary. We will come back to this point later.

Let us consider the one-loop integral of Eq. (2.2) with $M=0$ and $q=0$. By definition the resulting massless tadpole integral is set to zero in dimensional regularization. On the other hand, the application of the $R^{\star}$ operation gives

$$
\begin{aligned}
0 & =R^{\star}\left[\int_{1} \frac{1}{\left[-k_{1}^{2}\right]\left[-k_{1}^{2}\right]}\right] \\
& =\tilde{R}\left[\int_{1} \frac{1}{\left[-k_{1}^{2}\right]\left[-k_{1}^{2}\right]}+Z^{(1)}\right] \\
& =\int_{1} \frac{1}{\left[-k_{1}^{2}\right]\left[-k_{1}^{2}\right]}+\tilde{Z}^{(1)}+Z^{(1)} \\
& =\tilde{Z}^{(1)}+Z^{(1)} .
\end{aligned}
$$

In the first step the $R$ operation is applied resulting in the diagram itself and the counterterm $Z^{(1)}$ rendering the expression in the square brackets of the second line UV finite. The subsequent application of $\tilde{R}$ generates the counterterm $\tilde{Z}^{(1)}$ which corresponds to the IR divergence of one-loop integral. Once the application of $R^{\star}$ is resolved scaleless integrals are set to zero which leads to the last line of Eq. (2.6). Using Eq. (2.6) in combination with Eq. (2.3), one obtains for the IR renormalization constant of the one-loop two-point function

$$
\tilde{Z}^{(1)}=\frac{1}{16 \pi^{2}} \frac{1}{\varepsilon} .
$$

In analogy, applying the $R^{\star}$ operation to the diagram of Fig. 2.1 with all masses and external momenta set to zero leads to

$$
\begin{aligned}
0 & =R^{\star}\left[\int_{1} \int_{2} \frac{1}{\left[-k_{1}^{2}\right]\left[-k_{2}^{2}\right]^{2}\left[-\left(k_{1}-k_{2}\right)^{2}\right]}\right] \\
& =\tilde{R}\left[\int_{1} \int_{2} \frac{1}{\left[-k_{1}^{2}\right]\left[-k_{2}^{2}\right]^{2}\left[-\left(k_{1}-k_{2}\right)^{2}\right]}+Z^{(1)} \int_{2} \frac{1}{\left[-k_{2}^{2}\right]^{2}}+Z^{(2)}\right] \\
& =\tilde{Z}^{(2)}+Z^{(1)} \tilde{Z}^{(1)}+Z^{(2)} .
\end{aligned}
$$

This equation defines the IR renormalization constant $\tilde{Z}^{(2)}$.

Let us come back to the two-loop diagram of Fig. 2.1 with $m=0$. In this limit the diagram contains both an IR and UV divergent subdiagram. The application of $R^{\star}$ generates the following terms

$$
R^{\star}\left[\int_{1} \int_{2} \frac{1}{\left[M^{2}-\left(k_{1}-k_{2}\right)^{2}\right]\left[M^{2}-\left(k_{1}+q\right)^{2}\right]\left[-k_{2}^{2}\right]^{2}}\right]
$$




$$
\begin{aligned}
= & \tilde{R}\left[\int_{1} \int_{2} \frac{1}{\left[M^{2}-\left(k_{1}-k_{2}\right)^{2}\right]\left[M^{2}-\left(k_{1}+q\right)^{2}\right]\left[-k_{2}^{2}\right]^{2}}+Z^{(1)} \int_{2} \frac{1}{\left[-k_{2}^{2}\right]^{2}}+Z^{(2)}\right] \\
= & \int_{1} \int_{2} \frac{1}{\left[M^{2}-\left(k_{1}-k_{2}\right)^{2}\right]\left[M^{2}-\left(k_{1}+q\right)^{2}\right]\left[-k_{2}^{2}\right]^{2}}+\tilde{Z}^{(1)} \int_{1} \frac{1}{\left[M^{2}-k_{1}^{2}\right]\left[M^{2}-\left(k_{1}+q\right)^{2}\right]} \\
& +Z^{(1)} \tilde{Z}^{(1)}+Z^{(2)} \\
= & \left(\frac{1}{16 \pi^{2}}\right)^{2}\left\{\left[-\frac{1}{2 \varepsilon^{2}}-\frac{1}{2 \varepsilon}\left(-1+2 \ln \frac{\mu^{2}}{M^{2}}\right)\right]+\frac{1}{\varepsilon}\left(\frac{1}{\varepsilon}+\ln \frac{\mu^{2}}{M^{2}}\right)\right. \\
& \left.+\left(-\frac{1}{\varepsilon}\right) \frac{1}{\varepsilon}+\left[\frac{1}{2 \varepsilon^{2}}-\frac{1}{2 \varepsilon}\right]+\text { finite terms }\right\} \\
= & \text { finite, }
\end{aligned}
$$

where $\tilde{R}$ after the second equal sign acts on the IR divergent integral $\int_{2} 1 /\left[-k_{2}^{2}\right]^{2}$ and generates the factors $\tilde{Z}^{(1)}$. Note that no term $\tilde{Z}^{(2)}$ appears as the original integral involves the scales $M$ and $q$. After the third equal sign the results of Eqs. (2.3), (2.5), (2.7) and of the Appendix (A.4) have been used in order to explicitly show the finiteness. In general the logic is the other way around: one chooses the masses and external momenta in such a way that the very diagram, i.e. the first term in the second line of Eq. (2.9), can easily be evaluated and uses Eq. (2.9) in order to determine $Z^{(2)}$.

At the end of this Subsection we want to work out the connection of the $R^{\star}$ operation to the asymptotic expansion with respect to large masses. The hard-mass procedure provides a prescription on how to evaluate Feynman integrals where one of the internal masses is much larger than the others (cf. Appendix A.1). Thus one can adopt the point-of-view to introduce light masses which regularize all IR divergences and apply the hard-mass procedure. For illustration let us consider the diagram in Fig. 2.1 with one of the propagators carrying mass $M$ doubled in order to avoid UV divergences which keeps the formula more transparent. For $m \neq 0$ the diagram is also IR finite. Applying the hard-mass procedure in the limit $M^{2} \gg m^{2}, q^{2}$ and keeping only the leading terms in $m^{2} / M^{2}$ and $q^{2} / M^{2}$ leads to

$$
\begin{aligned}
& \int_{1} \int_{2} \frac{1}{\left[M^{2}-\left(k_{1}-k_{2}\right)^{2}\right]\left[M^{2}-\left(k_{1}+q\right)^{2}\right]^{2}\left[m^{2}-k_{2}^{2}\right]^{2}} \\
& \quad=\int_{1} \int_{2} \frac{1}{\left[M^{2}-\left(k_{1}-k_{2}\right)^{2}\right]\left[M^{2}-k_{1}^{2}\right]^{2}\left[-k_{2}^{2}\right]^{2}}+\int_{1} \frac{1}{\left[M^{2}-k_{1}^{2}\right]^{3}} \int_{2} \frac{1}{\left[m^{2}-k_{2}^{2}\right]^{2}}+\ldots,
\end{aligned}
$$

where the ellipses represent terms of order $q^{2} / M^{2}$ and $m^{2} / M^{2}$. The general rules and explicit examples for the hard-mass procedure are discussed in Appendix A.1. The application of Eq. (A.2) to the two-loop diagram at hand leads to the two contributions which are listed in the second line of Eq. (2.10). The first term corresponds to the whole diagram which according to the rules of the hard-mass procedure has to be expanded in the small quantities $q$ and $m$. To our approximation this means to simply nullify both $q$ and $m$. In the second contribution the hard subgraph consists of the one-loop subdiagram where all lines carry the heavy mass $M$. The expansion in the external momenta leads to 
$\int_{1} 1 /\left[M^{2}-k_{1}^{2}\right]^{3}$ which finally leads to the second term on the right-hand side of Eq. (2.10). Note that the first integral is IR divergent whereas the second one is UV divergent. Their connection to the $R$ and $\tilde{R}$ operation becomes clear after adding and subtracting the term $Z^{(1)} \int_{1} 1 /\left[M^{2}-k_{1}^{2}\right]^{3}$

$$
\begin{aligned}
\text { Eq. (2.10) }= & \int_{1} \int_{2} \frac{1}{\left[M^{2}-\left(k_{1}-k_{2}\right)^{2}\right]\left[M^{2}-k_{1}^{2}\right]^{2}\left[-k_{2}^{2}\right]^{2}}-\int_{1} \frac{1}{\left[M^{2}-k_{1}^{2}\right]^{3}} Z^{(1)} \\
& +\int_{1} \frac{1}{\left[M^{2}-k_{1}^{2}\right]^{3}}\left(\int_{2} \frac{1}{\left[m^{2}-k_{2}^{2}\right]^{2}}+Z^{(1)}\right)+\ldots \\
= & \tilde{R}\left[\int_{1} \int_{2} \frac{1}{\left[M^{2}-\left(k_{1}-k_{2}\right)^{2}\right]\left[M^{2}-k_{1}^{2}\right]^{2}\left[-k_{2}^{2}\right]^{2}}\right] \\
& +\int_{1} \frac{1}{\left[M^{2}-k_{1}^{2}\right]^{3}} R\left[\int_{2} \frac{1}{\left[m^{2}-k_{2}^{2}\right]^{2}}\right]+\ldots,
\end{aligned}
$$

where in the last step Eq. (2.6) has been used. In Eq. (2.11) the correspondence between the IR and UV divergences, which are introduced through the hard-mass procedure, can nicely be observed. Furthermore, it can be seen how they have to be combined in order to arrive at the final form which contains the application of the $R$ and $\tilde{R}$ operations.

\subsection{Global infra-red re-arrangement and the quark anomalous dimension}

In the previous Subsection it has been demonstrated that the IRR in connection with the $R^{\star}$ operation provides a very powerful tool for the computation of renormalization group functions at higher orders. In this Subsection we want to discuss its practical application in the case of the quark anomalous dimension $\gamma_{m}$.

In the $\overline{\mathrm{MS}}$ scheme the running of the quark masses is governed by the function $\gamma_{m}\left(\alpha_{s}\right)$

$$
\mu^{2} \frac{d}{d \mu^{2}} m^{\left(n_{f}\right)}(\mu)=m^{\left(n_{f}\right)}(\mu) \gamma_{m}^{\left(n_{f}\right)}\left(\alpha_{s}^{\left(n_{f}\right)}\right)=-m^{\left(n_{f}\right)}(\mu) \sum_{i \geq 0} \gamma_{m, i}^{\left(n_{f}\right)}\left(\frac{\alpha_{s}^{\left(n_{f}\right)}(\mu)}{\pi}\right)^{i+1},
$$

where the coefficients $\gamma_{m, i}$ are known up to the four-loop order [32, 33, 34, 15, 16]

$$
\begin{aligned}
\gamma_{m, 0}^{\left(n_{f}\right)}= & 1 \\
\gamma_{m, 1}^{\left(n_{f}\right)}= & \frac{1}{16}\left[\frac{202}{3}-\frac{20}{9} n_{f}\right] \\
\gamma_{m, 2}^{\left(n_{f}\right)}= & \frac{1}{64}\left[1249+\left(-\frac{2216}{27}-\frac{160}{3} \zeta_{3}\right) n_{f}-\frac{140}{81} n_{f}^{2}\right], \\
\gamma_{m, 3}^{\left(n_{f}\right)}= & \frac{1}{256}\left[\frac{4603055}{162}+\frac{135680}{27} \zeta_{3}-8800 \zeta_{5}+\left(-\frac{91723}{27}-\frac{34192}{9} \zeta_{3}+880 \zeta_{4}\right.\right. \\
& \left.+\frac{18400}{9} \zeta_{5}\right) n_{f}+\left(\frac{5242}{243}+\frac{800}{9} \zeta_{3}-\frac{160}{3} \zeta_{4}\right) n_{f}^{2}
\end{aligned}
$$




$$
\left.+\left(-\frac{332}{243}+\frac{64}{27} \zeta_{3}\right) n_{f}^{3}\right]
$$

with $\zeta_{3} \approx 1.202057, \zeta_{4}=\pi^{4} / 90$ and $\zeta_{5} \approx 1.036928$. The superscript $n_{f}$ indicates the dependence on the number of quarks.

For the computation of $\gamma_{m}$ one has to know the quark mass renormalization constant in the $\overline{\mathrm{MS}}$ scheme, $Z_{m}$, which relates the bare mass, $m^{0}$, to the renormalized one through

$$
m^{0}=Z_{m} m
$$

$Z_{m}$ can be obtained form the vector and scalar parts of the quark propagator. Its inverse reads in bare form

$$
\begin{aligned}
\left(S_{F}^{0}(q)\right)^{-1} & =i\left[m^{0}-\not 1-\Sigma^{0}(q)\right] \\
& =i\left[m^{0}\left(1-\Sigma_{S}^{0}\right)-\not q\left(1+\Sigma_{V}^{0}\right)\right],
\end{aligned}
$$

where the functions $\Sigma_{S}^{0}$ and $\Sigma_{V}^{0}$ depend on the external momentum $q$, the bare mass $m^{0}$ and on the bare strong coupling constant $\alpha_{s}^{0}$. From the requirement that the renormalized quark propagator is finite one gets in the $\overline{\mathrm{MS}}$ scheme the following two equations

$$
\begin{aligned}
Z_{2} & =1-K_{\varepsilon}\left[Z_{2} \Sigma_{V}^{0}\right], \\
Z_{2} Z_{m} & =1+K_{\varepsilon}\left[Z_{2} Z_{m} \Sigma_{S}^{0}\right],
\end{aligned}
$$

where $Z_{2}$ is the wave function renormalization of the quark. The operator $K_{\varepsilon}$ extracts the poles in $1 / \varepsilon$. The Eqs. (2.16) are solved recursively for $Z_{2}$ and $Z_{m}$, and $\gamma_{m}$ is computed from Eq. (2.12) using (2.14) and the fact that $\mu^{2} \mathrm{~d} m_{0} / \mathrm{d} \mu^{2}=0$. This leads to

$$
\begin{aligned}
\gamma_{m} & =-\mu^{2} \frac{\mathrm{d}}{\mathrm{d} \mu^{2}} \ln Z_{m} \\
& =-\beta^{\varepsilon}\left(\alpha_{s}\right) \pi \frac{\partial}{\partial \alpha_{s}} \ln Z_{m},
\end{aligned}
$$

where $\beta^{\varepsilon}\left(\alpha_{s}\right)=-\varepsilon+\beta\left(\alpha_{s}\right)$ is the $D$-dimensional $\beta$ function and $\beta\left(\alpha_{s}\right)$ is defined below in Eq. (2.35).

For the computation in the $\overline{\mathrm{MS}}$ scheme one has to evaluate the pole part of the fermion propagator. Some sample diagrams contributing at one, two, three and four loops are shown in Fig. 2.2. As the diagrams contributing to $\Sigma_{S}^{0}$ and $\Sigma_{V}^{0}$ are logarithmically divergent the computation can be performed by setting all internal masses to zero and keeping the external momentum finite. This leads to massless l-loop propagator-type integrals for the evaluation of $\gamma_{m, l-1}$. All occuring integrals are free from IR divergences. At one-, two- and three-loop order the package MINCER [31] can be used in order to perform the computation. However, this method fails to be practical for the computation of $\gamma_{m, 3}$ as currently massless four-loop integrals are not available. Alternatively one could set the external momentum to zero but keep the quark mass finite. Again the technology is 


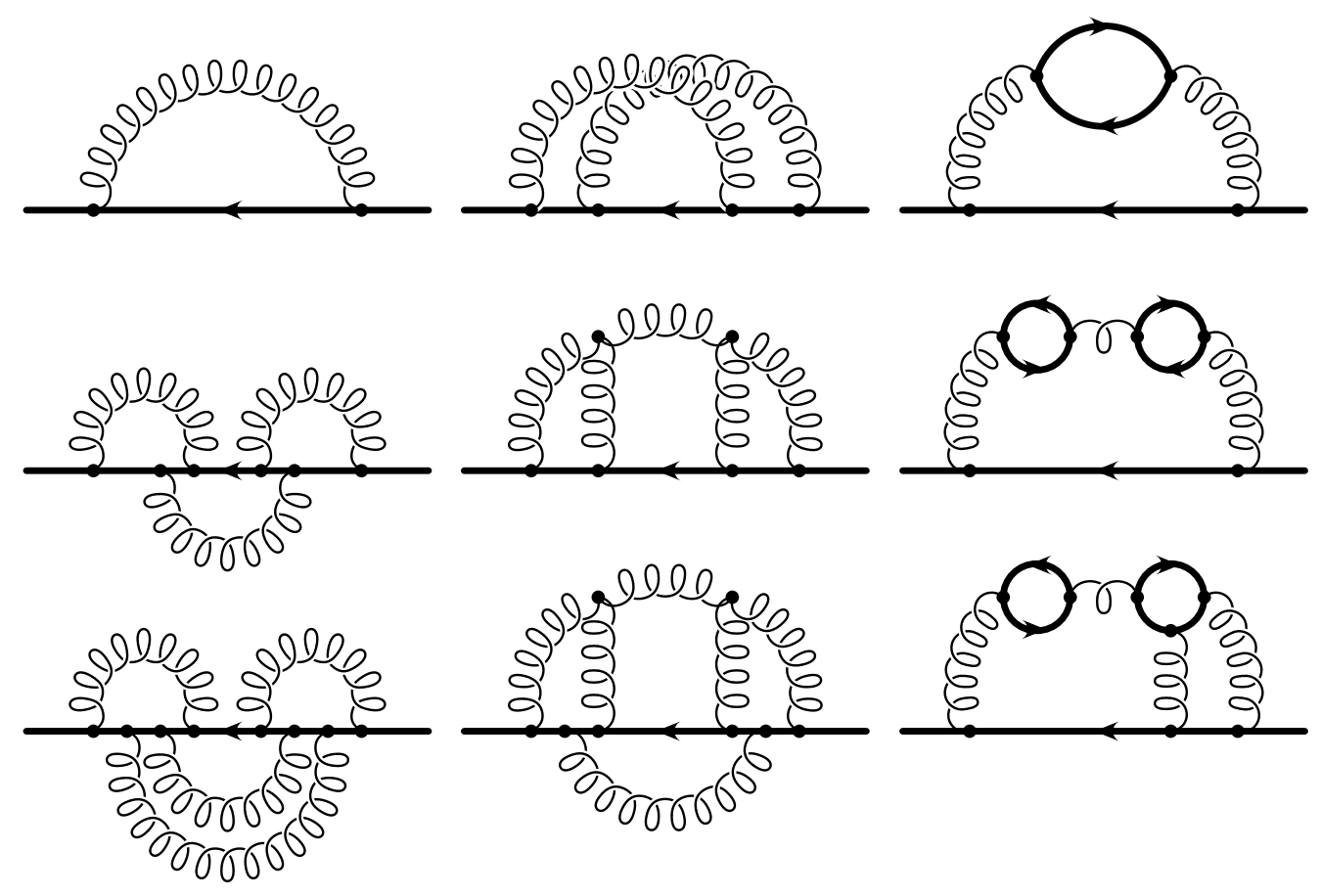

Figure 2.2: Sample diagrams contributing to the fermion propagator.

available to perform the three-loop calculation [35], however, the corresponding four-loop vacuum diagrams are currently again out of range.

A tempting approach for the computation of $\gamma_{m, 3}$, which actually was considered in [15] (see also [36]), is the following: due to the properties of the IRR [26] one can set to zero the external momentum and choose an arbitrary subset of the internal lines to have a non-zero mass. A clever choice is to allow only the quark propagator which is attached to the left vertex a non-zero mass $M$. This has the advantage that the $l$-loop integrals can be solved by iterating a massive one-loop vacuum and a $(l-1)$-loop massless propagatortype integral where the external momentum of the latter exactly corresponds to the loop momentum of the former. Thus, even at four-loop order at most massless three-loop two-point integrals have to be evaluated. It is sufficient to compute their finite parts, as only the $1 / \varepsilon$ poles of the l-loop integral are needed. However, there are two subtleties connected to this choice of IR structure. The first one is connected to the asymmetry one introduces due to the choice of the massive line. As a consequence the "left" vertex has to be renormalized differently from the "right" one. This we will explicitly see in the formulae we derive below.

The second disadvantage is the occurrence of IR divergences. At this point the idea is to use the $R^{\star}$ operation in order to subtract them. However, an effective evaluation of the roughly 6000 diagrams is only possible if the computation of the IR renormalization constant can be performed in a global way. This was achieved in Ref. 15 and will be 
described in the following.

As the explicit formulae for $Z_{2}$ and $Z_{m}$ are not yet available in the literature and as they will be published elsewhere [37] we present the derivation of a formula for the renormalization constant of the vector current correlator, $Z^{\mathrm{em}}$, which is quite similar to the one of $Z_{2}$ and $Z_{m}$. $Z^{\mathrm{em}}$ evaluated at four-loop order immediately leads to corrections of order $\alpha_{s}^{3}$ to the cross section $\sigma\left(e^{+} e^{-} \rightarrow\right.$ hadrons) [38].

Our starting point for the computation of $Z^{\mathrm{em}}$ is the renormalized vector current polarization functionf which can be cast in the form

$$
\Pi^{\mu \nu}(q)=\left(\frac{Z_{V}}{Z_{2}}\right)^{2} \Pi^{0, \mu \nu}(q)+Z^{\mathrm{em}}\left(-q^{2} g^{\mu \nu}+q^{\mu} q^{\nu}\right) .
$$

$\Pi^{0, \mu \nu}(q)$ is the bare correlator as indicated by the index "0". $Z_{2}$ corresponds to the wave function renormalization constant and $Z_{V}$ is the renormalization constant for the vector current $j^{v}=\bar{\psi} \gamma^{\mu} \psi$ defined through

$$
\left.\left(j^{v}\right)\right|_{\text {ren. }}=\left.\frac{Z_{V}}{Z_{2}}\left(j^{v}\right)\right|_{\text {bare }} .
$$

Note that $Z^{\mathrm{em}}$ as defined in Eq. (2.18) receives contributions starting from one-loop order.

In order to derive a formula for the computation of $Z^{\mathrm{em}}$ we use for $\Pi^{0, \mu \nu}(q)$ a DysonSchwinger-type representation containing the full fermion propagator, $G^{0}$, and the proper photon-quark vertex function, $\Gamma^{0, \mu}$, leaving out one integration over the final loop momentum which we call $p$. The resulting diagrammatic representation is visualized in Fig. 2.3(a). After contraction with $g_{\mu \nu}$ one obtains

$$
\begin{aligned}
\Pi_{\mu}^{\mu}(q)= & -\left(\frac{Z_{V}}{Z_{2}}\right)^{2} \operatorname{Tr}\left[\int \frac{\mathrm{d}^{D} p}{(2 \pi)^{D}} i \gamma_{\mu} G^{0}\left(p, \alpha_{s}^{0}\right) \Gamma^{0, \mu}\left(p, q, \alpha_{s}^{0}\right) G^{0}\left(p+q, \alpha_{s}^{0}\right)\right] \\
& +Z^{\mathrm{em}} q^{2}(1-D),
\end{aligned}
$$

where the minus sign in front of the first term on the right hand side accounts for the closed fermion loop corresponding to the $p$ integration. In Eq. (2.20) all colour indices have been suppressed and the dependence of $G^{0}$ and $\Gamma^{0, \mu}$ on the bare coupling $\alpha_{s}^{0}$ is made explicit.

Next we exploit the finiteness of $\Pi_{\mu}^{\mu}$ in Eq. (2.18) and the fact that $Z^{\mathrm{em}}$ only contains poles in $1 / \varepsilon$. This leads to an explicit formula for $Z^{\mathrm{em}}$ which reads

$$
\begin{aligned}
Z^{\mathrm{em}}(D-1)=-K_{\varepsilon}\left\{( \frac { Z _ { V } } { Z _ { 2 } } ) ^ { 2 } \frac { \square _ { q } } { 2 D } \operatorname { T r } \left[\int \frac{\mathrm{d}^{D} p}{(2 \pi)^{D}} i \gamma_{\mu}\right.\right. \\
\\
\left.\left.G^{0}\left(p, \alpha_{s}^{0}\right) \Gamma^{0, \mu}\left(p, q, \alpha_{s}^{0}\right) G^{0}\left(p+q, \alpha_{s}^{0}\right)\right]\left.\right|_{q \rightarrow 0}\right\} .
\end{aligned}
$$

In this equation we consider the limit $q \rightarrow 0$ on the right-hand side. This is possible as in the $\overline{\mathrm{MS}}$ scheme $Z^{\mathrm{em}}$ does not depend on any dimensional scale. In order to evaluate

\footnotetext{
${ }^{4}$ For a precise definition see Eq. (5.15).
} 


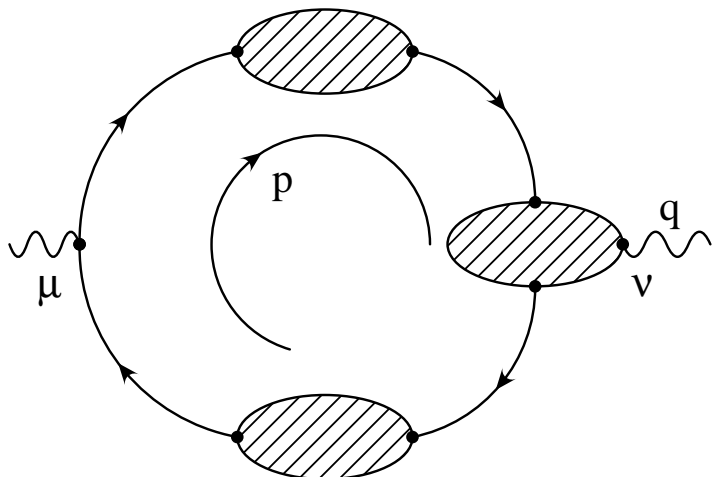

(a)

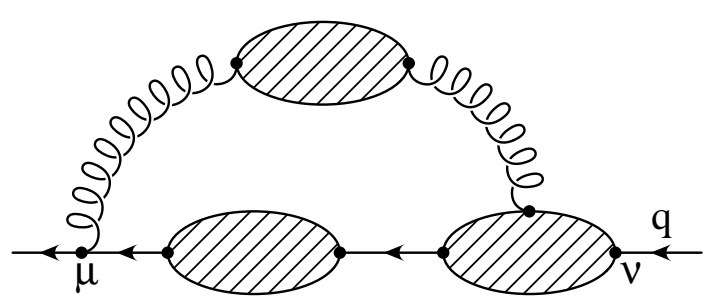

(b)

Figure 2.3: (a) Graphical representation of Eq. (2.20). In the case of the fermion propagator the analogous diagram looks like the one in (b). In both cases an artificial mass is introduced in the propagator of the fermion line attached to the left vertex.

the four-loop contribution to the polarization function $G^{0}$ and $\Gamma^{0, \mu}$ have to be inserted at tree-level and at one-, two- and three-loop order.

Let us next have a closer look to the renormalization constant $Z_{V}$. For the "right" vertex we have $Z_{V}=Z_{2}$. However, due to the artificial mass which we want to introduce in the fermion line connected to the "left" vertex the equation $Z_{V}=Z_{2}$ can not be applied immediately. Instead we write for the "left" vertex $Z_{V}=1+\delta Z_{V}$ and thus obtain from Eq. (2.21)

$$
\begin{aligned}
Z^{\mathrm{em}}(D-1)= & -K_{\varepsilon}\left\{\frac { 1 } { Z _ { 2 } } \frac { \square _ { q } } { 2 D } \operatorname { T r } \left[\int \frac{\mathrm{d}^{D} p}{(2 \pi)^{D}} i \gamma_{\mu} \frac{p^{2}}{p^{2}-M^{2}}\right.\right. \\
& \left.G^{0}\left(p, \alpha_{s}^{0}\right) \Gamma^{0, \mu}\left(p, q, \alpha_{s}^{0}\right) G^{0}\left(p+q, \alpha_{s}^{0}\right)\right]\left.\right|_{q \rightarrow 0} \\
& \left.+\left.\frac{\delta Z_{V}}{Z_{2}} \frac{\square_{q}}{2 D} \operatorname{Tr}\left[\int \frac{\mathrm{d}^{D} p}{(2 \pi)^{D}} i \gamma_{\mu} G^{0}\left(p, \alpha_{s}^{0}\right) \Gamma^{0, \mu}\left(p, q, \alpha_{s}^{0}\right) G^{0}\left(p+q, \alpha_{s}^{0}\right)\right]\right|_{q \rightarrow 0}\right\} .
\end{aligned}
$$

In the first line the presence of the artificial mass is made explicit in the factor $p^{2} /\left(p^{2}-M^{2}\right)$ which effectively replaces the massless propagator by a massive one. On the other hand, in the second equation $M$ can be set to zero, as $\delta Z_{V}$ already contains the effect of $M$. In a next step we want to perform the limit $q \rightarrow 0$. In particular, we use $M^{2} \gg q^{2}$ and apply the hard-mass procedure to the first term in Eq. (2.22) which transforms it to

$$
\begin{aligned}
Z^{\mathrm{em}}(D-1)=-K_{\varepsilon}\left\{\frac { 1 } { Z _ { 2 } } \frac { \square _ { q } } { 2 D } \operatorname { T r } \left[\int \frac{\mathrm{d}^{D} p}{(2 \pi)^{D}} i \gamma_{\mu} \frac{p^{2}}{p^{2}-M^{2}}\right.\right. \\
\left.G^{0}\left(p, \alpha_{s}^{0}\right) \Gamma^{0, \mu}\left(p, q, \alpha_{s}^{0}\right) G^{0}\left(p+q, \alpha_{s}^{0}\right)\right]\left.\right|_{q=0}
\end{aligned}
$$




$$
\begin{gathered}
+\frac{1}{Z_{2}} \tilde{R}\left[\Gamma _ { f \overline { f } \gamma } ( 0 , 0 ) \frac { \square _ { q } } { 2 D } \operatorname { T r } \left[\int \frac{\mathrm{d}^{D} p}{(2 \pi)^{D}} i \gamma_{\mu}\right.\right. \\
\left.\left.G^{0}\left(p, \alpha_{s}^{0}\right) \Gamma^{0, \mu}\left(p, q, \alpha_{s}^{0}\right) G^{0}\left(p+q, \alpha_{s}^{0}\right)\right]\left.\right|_{q=0}\right] \\
+\frac{\delta Z_{V}}{Z_{2}} \tilde{R}\left[\frac { \square _ { q } } { 2 D } \operatorname { T r } \left[\int \frac{\mathrm{d}^{D} p}{(2 \pi)^{D}} i \gamma_{\mu}\right.\right. \\
\left.\left.\left.G^{0}\left(p, \alpha_{s}^{0}\right) \Gamma^{0, \mu}\left(p, q, \alpha_{s}^{0}\right) G^{0}\left(p+q, \alpha_{s}^{0}\right)\right]\left.\right|_{q=0}\right]\right\} .
\end{gathered}
$$

where $\gamma^{\mu} \delta \Gamma_{f \bar{f} \gamma}(0,0)$ represents the sum of all one-particle-irreducible (1PI) hard subgraphs containing the mass $M$. It gets contributions starting from one-loop order. In Eq. (2.23) we introduced the $\tilde{R}$ operation in order to treat the IR divergences of those terms which lead to massless tadpoles for $q \rightarrow 0$. Note that in general also the first term in (2.23) contains IR divergences. However, they originate from the hard-mass procedure and are correlated to the UV poles of the second term in Eq. (2.23) (cf. the discussion around Eq. (2.11)).

The IR divergences which occur in the last two terms of Eq. (2.23) for $q=0$ are taken care by introducing appropriate renormalization constants. They can be determined in a global manner by setting $q=0$ in Eq. (2.21) and applying the $\tilde{R}$ operation. As the left-hand side is unaffected we have

$$
\begin{aligned}
Z^{\mathrm{em}}(D-1)=-K_{\varepsilon}\left\{\tilde { R } \left[\frac { \square _ { q } } { 2 D } \operatorname { T r } \left[\int \frac{\mathrm{d}^{D} p}{(2 \pi)^{D}} i \gamma_{\mu}\right.\right.\right. \\
\\
\left.\left.\left.\quad G^{0}\left(p, \alpha_{s}^{0}\right) \Gamma^{0, \mu}\left(p, q, \alpha_{s}^{0}\right) G^{0}\left(p+q, \alpha_{s}^{0}\right)\right]\left.\right|_{q=0}\right]\right\} .
\end{aligned}
$$

The application of $\tilde{R}$ generates only one term, namely the IR counterterm, as we set massless tadpoles to zero. This counterterm is a global one as it treats the IR divergences of the sum of all diagrams. We want to mention that this convenient aspect is new as compared to older calculations where the $\tilde{R}$ operator has been used. In Ref. [39], e.g., the $\tilde{R}$ operator has been applied to each diagram individually which in practice is quite tedious.

Inserting Eq. (2.24) into (2.23) finally leads to

$$
\begin{aligned}
& Z^{\mathrm{em}}(D-1)=-K_{\varepsilon}\left\{\frac { 1 } { Z _ { 2 } } \frac { \square _ { q } } { 2 D } \operatorname { T r } \left[\int \frac{\mathrm{d}^{D} p}{(2 \pi)^{D}} i \gamma_{\mu} \frac{p^{2}}{p^{2}-M^{2}}\right.\right. \\
&\left.G^{0}\left(p, \alpha_{s}^{0}\right) \Gamma^{0, \mu}\left(p, q, \alpha_{s}^{0}\right) G^{0}\left(p+q, \alpha_{s}^{0}\right)\right]\left.\right|_{q=0} \\
&\left.+\frac{(1-D) Z^{\mathrm{em}}}{Z_{2}}\left(\delta \Gamma_{f \bar{f} \gamma}(0,0)+\delta Z_{V}\right)\right\} .
\end{aligned}
$$


Note that at this point the relation $\delta Z_{V}=Z_{2}-1$ can be used.

At one-loop order only the first line of Eq. (2.25) contributes where all renormalization constants can be set to one. Furthermore the functions $G^{0}$ and $\Gamma^{0, \mu}$ take their tree-level values and only the one-loop diagram shown in Fig. 2.4 has to be evaluated for external momentum zero and finite quark mass in one of the fermion lines. In this case no IR divergences occur.
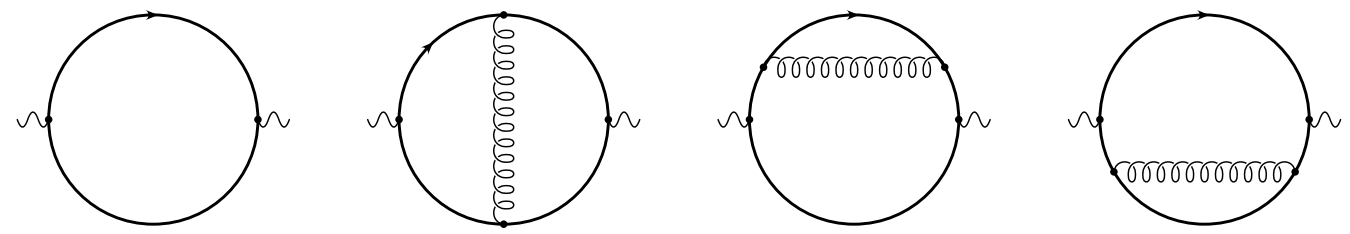

Figure 2.4: One- and two-loop diagrams contributing the vector current correlator.

A non-trivial contribution from the terms in the second line of Eq. (2.25) occurs for the first time at two-loop order. In particular, for $\delta \Gamma_{f \bar{f} \gamma}$ the one-loop photon-fermion vertex corrections have to be evaluated where one of the quarks carries mass $M$ and the other one is massless. All external momenta equal to zero. In the three two-loop diagrams (see Fig. 2.4) contributing to the first line we consequently assign a mass $M$ to the fermion (not the anti-fermion) line attached to the "left" vertex and set the masses in all other propagators to zero. For vanishing external momentum in each diagram a massless oneloop two-point function can be identified which can be solved with the help of Eq. (A.4) in Appendix A.2. The external momentum coincides with the loop momentum of the remaining one-loop vacuum integral which is also expressible in terms of $\Gamma$ functions (cf. Eq. (A.6)).

In general, for a $l$-loop calculation the renormalization constants occuring in Eq. (2.25) are only needed at loop order $(l-1)$. The loop integrals which are necessary to compute the first expression in Eq. (2.25) are massless $(l-1)$-loop two-point functions and one-loop vacuum integrals. For the evaluation of $\delta \Gamma_{f \bar{f} \gamma},(l-1)$-loop vacuum integral are needed. In particular, for $l=4$ the occuring integrals are very well studied (cf. Refs. [31, 35]).

In the above equations the bare coupling constant is used as a parameter. It has to be expressed through the renormalized version using the $(l-1)$-loop formula for the renormalization constant $Z_{g}$ (cf. Eq. (3.3)). We want to note that in our case the mass $M$ needs not to be renormalized as in the diagrams no subdivergence is present which could induce a corresponding counterterm.

Besides the application to the vector current correlator, which leads to corrections of order $\alpha_{s}^{3}$ to $R(s)$ [38, the method described in this Subsection has successfully been applied to the four-loop fermion propagator and the correlator of scalar currents in order to evaluate the the four-loop contribution to $\gamma_{m}$ [15] and corrections of order $\alpha_{s}^{3}$ to the decay of a scalar Higgs boson [40], respectively.

Due to the large number of genuine four-loop diagrams an automation of the compu- 
tation is mandatory. The four-loop calculations of [38, 40, 15] have been performed with the help of the package GEFICOM [41]. Within this framework the computation of $\delta \Gamma_{f \bar{f} \gamma}$ is straightforward as one- two- and three-loop vacuum integrals are directly accessible. For the four-loop contributions, like in the first line of Eq. (2.25), some tricks are necessary. In particular completely massless four-loop diagrams are generated. In a next step a Mathematica [42] program is used to identify the "left" vertex and to introduce the mass $M$. At this point the topology of the massless three-loop diagram is fixed and the mapping of the momenta according to the notation of MINCER [31] can be performed. One has to exploit that the whole diagram is logarithmically divergent and the mass dimension is given by the artificial mass $M$. Then the massless integration and at the end also the one-loop massive one can be performed.

\subsection{Massive vacuum integrals and the $\beta$ function}

A different approach has been employed in [14, 16] in order to compute the four-loop contribution to the $\beta$ and $\gamma_{m}$ functions. Also here the basic property of the IRR has been exploited and the integrals have been simplified by modifying the IR behaviour of the Feynman diagrams. This time, however, a different attitude has been adopted than in the previous section: in all denominators, also the ones of the gluons and ghosts, a common mass parameter, $M$, has been introduced (see also 43]). This avoids completely the IR divergences. On the other side, however, both gauge invariance and useful Ward identities are broken by this method. Moreover, multiplicative renormalization, which is very convenient in practical renormalization is lost. Nevertheless the $R$ operation applied to the individual diagrams still works and in principle it can be used to compute the overall renormalization constant. However, this is not at all practical, especially as in the case of the $\beta$ function roughly 50000 diagrams have to be considered [14] and an automatic treatment is absolutely mandatory. This is achieved by introducing effective vertices and propagators which incorporate all lower-order renormalization constants [14]. Furthermore counterterms have to be introduced which correspond to a renormalization of the gluon and ghost mass and an overall renormalization constant for the gluon propagator.

In the following we want to illustrate this method by considering two-loop QCD corrections to the photon propagator and evaluate the corrections to the wave function renormalization defined through

$$
A^{0, \mu}=\sqrt{Z_{3}^{\gamma}} A^{\mu}
$$

where $A^{\mu}$ is the photon field. For convenience we also introduce the renormalization constants $Z_{1}^{\gamma}$ and $Z_{2}^{\gamma}$ via

$$
\begin{aligned}
\psi^{0} & =\sqrt{Z_{2}^{\gamma}} \psi \\
e^{0} \bar{\psi}^{0} \gamma^{\mu} \psi^{0} A_{\mu}^{0} & =Z_{1}^{\gamma} e \bar{\psi} \gamma^{\mu} \psi A_{\mu},
\end{aligned}
$$

where $e$ is the electromagnetic charge and $\psi$ is the fermion field. The corrections up to order $\alpha \alpha_{s}$ to $Z_{3}^{\gamma}$ are obtained from the diagrams of Fig. 2.4 which contribute to the photon 
polarization function. The latter can be written in the form

$$
\Pi^{\mu \nu}(q)=\left(-g^{\mu \nu}+\frac{q^{\mu} q^{\nu}}{q^{2}}\right) \Sigma_{T}\left(q^{2}\right)+\frac{q^{\mu} q^{\nu}}{q^{2}} \Sigma_{L}\left(q^{2}\right) .
$$

The canonical way to compute $Z_{3}^{\gamma}$ would be to consider all particles as massless and evaluate the occuring massless two-point function in dimensional regularization. In this case $\Sigma_{T}\left(q^{2}\right)$ is proportional to $q^{2}$ and $\Sigma_{L}\left(q^{2}\right) \equiv 0$ which is due to gauge invariance. One observes that the sum of the bare two-loop diagrams only contains a simple pole in $\varepsilon$ and that it is independent of the QCD gauge parameter, $\xi$. Furthermore, due to the Ward identity connecting the photon-quark vertex to the quark self energy no renormalization is necessary and the bare diagrams of Fig. 2.4 already lead to the final answer where the formula

$$
Z_{3}^{\gamma}=1-K_{\varepsilon}\left(\frac{\Sigma_{T}\left(q^{2}\right)}{q^{2}} Z_{3}^{\gamma}\right)
$$

can be used.

In the method proposed in [14, 16, 43] a common mass $M$ is introduced in all lines for the computation of the renormalization group functions. As a consequence gauge invariance is broken and $\Sigma_{L}\left(q^{2}\right)$ is not zero any more. Furthermore, both $\Sigma_{T}\left(q^{2}\right)$ and $\Sigma_{L}\left(q^{2}\right)$ contain terms proportional to $M^{2}$. One also observes that the sum of the bare diagrams contains a pole of the form $\xi / \varepsilon$. Clearly the $R$ operation applied to the individual diagrams would still lead to the correct answer. However, we want to "immitate" multiplicative renormalization as close as possible and introduce effective vertices and propagators. In the case of the photon-quark vertex we write

$$
\gamma^{\mu} \longrightarrow \gamma^{\mu} Z_{1}^{\gamma}=\gamma^{\mu}\left(1+\delta Z_{1}^{\gamma}\right) .
$$

which actually corresponds to multiplicative renormalization. The quark propagator is modified to

$$
\frac{\not p}{p^{2}-M^{2}} \longrightarrow \frac{\not p}{p^{2}-M^{2}}\left(1-\not p \delta Z_{2}^{\gamma} \frac{\not p}{p^{2}-M^{2}}\right) .
$$

Note that for $M=0$ the terms proportional to $\delta Z_{1}^{\gamma}$ and $\delta Z_{2}^{\gamma}$ are proportional to the Born diagram and a cancellation takes place due to the Ward identity $Z_{1}^{\gamma}=Z_{2}^{\gamma}$. For $M \neq 0, Z_{1}^{\gamma}$ and $Z_{2}^{\gamma}$ have to be determined form the vertex correction and quark selfenergy, respectively. To our approximation they take the same values as for $M=0$, namely

$$
Z_{1}^{\gamma}=Z_{2}^{\gamma}=1+\frac{\alpha_{s}}{\pi} \frac{C_{F}}{\varepsilon}\left(\frac{1}{4} \xi-\frac{1}{4}\right),
$$

where $\xi$ is the gauge parameter defined in Eq. (3.2).

The use of Eqs. (2.30) and (2.31) for the computation of the one-loop diagram of Fig. 2.4 induces terms of order $\alpha \alpha_{s}$ which render the pole part independent of $\xi$. However, 
$\Pi^{\mu \nu}$ still contains terms proportional to $g^{\mu \nu} M^{2}$. They are removed by introducing a local mass counterterm for the photon of the form $M^{2} A_{\mu} A^{\mu}$. Also for the gluon field a similar counterterm has to be introduced. It is contained in the effective gluon propagator as it is needed for the cancellation of subdivergences.

Finally one arrives at

$$
Z_{3}^{\gamma}=1-\frac{\alpha}{\pi}\left(\frac{1}{3 \varepsilon}+\frac{\alpha_{s}}{\pi} C_{F} \frac{1}{8 \varepsilon}\right)
$$

In Ref. [14 this method has been applied to obtain the four-loop contribution of the QCD $\beta$ function. It has been applied to the ghost-gluon vertex, the gluon propagator and the ghost propagator in order to obtain the corresponding renormalization constants $\tilde{Z}_{1}$, $Z_{3}$ and $\tilde{Z}_{3}$ (cf. Eq. (3.3)) and finally the one for $\alpha_{s}$ via

$$
Z_{g}=\frac{\tilde{Z}_{1}}{\tilde{Z}_{3} \sqrt{Z_{3}}}
$$

The $\beta$ function is defined through

$$
\mu^{2} \frac{\mathrm{d}}{\mathrm{d} \mu^{2}} \frac{\alpha_{s}^{\left(n_{f}\right)}(\mu)}{\pi}=\beta^{\left(n_{f}\right)}\left(\alpha_{s}^{\left(n_{f}\right)}\right)=-\sum_{i \geq 0} \beta_{i}^{\left(n_{f}\right)}\left(\frac{\alpha_{s}^{\left(n_{f}\right)}(\mu)}{\pi}\right)^{i+2}
$$

where $n_{f}$ is the number of active flavours. For completeness we want to list the results for the coefficients which are given by [44, 45, 46, 14]

$$
\begin{aligned}
\beta_{0}^{\left(n_{f}\right)}= & \frac{1}{4}\left[11-\frac{2}{3} n_{f}\right] \\
\beta_{1}^{\left(n_{f}\right)}= & \frac{1}{16}\left[102-\frac{38}{3} n_{f}\right] \\
\beta_{2}^{\left(n_{f}\right)}= & \frac{1}{64}\left[\frac{2857}{2}-\frac{5033}{18} n_{f}+\frac{325}{54} n_{f}^{2}\right] \\
\beta_{3}^{\left(n_{f}\right)}= & \frac{1}{256}\left[\frac{149753}{6}+3564 \zeta_{3}+\left(-\frac{1078361}{162}-\frac{6508}{27} \zeta_{3}\right) n_{f}\right. \\
& \left.+\left(\frac{50065}{162}+\frac{6472}{81} \zeta_{3}\right) n_{f}^{2}+\frac{1093}{729} n_{f}^{3}\right] .
\end{aligned}
$$

$\zeta$ is Riemann's zeta function, with values $\zeta_{2}=\pi^{2} / 6$ and $\zeta_{3} \approx 1.202057$.

One of the main new achivements of Ref. [14] is the treatment of the four-loop vacuum diagrams. The task is simplified due to the fact that only the divergent parts in $\varepsilon$ are needed. The method of integration-by-parts 47 has been used to derive recurrence relations which reduce a general four-loop integral to a linear combination of simple integrals and two (difficult) master integrals. 


\section{Decoupling of heavy particles}

Quantum corrections to processes involving only light degrees of freedom contain in general the whole particle spectrum. In particular also heavy particles with masses much larger than the energy scale of the considered process contribute. It is highly desirable that in the limit where the heavy mass, $M$, goes to infinity its contribution to the lightparticle Green function must tend to zero like $\mu / M$ where $\mu$ is a typical scale of the process. This is exactly the content of the so-called decoupling theorem which is proven in 48 .

To be more precise let us consider an example, namely the production of heavy quarks in $e^{+} e^{-}$annihilation. Due to the hierarchy in the quark masses there is a clear separation into light and heavy. For center-of-mass energies, $\sqrt{s}$, of about $40 \mathrm{GeV}$ we are well below the production threshold of top quarks and thus we expect their influence to be suppressed by $\sqrt{s} / M_{t}$. Analogously the contribution of bottom quarks to the production of charm quarks close to the threshold must be proportional to $\sqrt{s} / M_{b}$. In our example the heavy quarks enter the first time at order $\alpha_{s}^{2}$ via the diagram pictured in Fig. 3.1. If the contribution of this diagram is computed in a momentum-subtraction scheme one indeed observes this behaviourf. However, in this example, and also in most other QCD processes it is much more convenient to use a mass-independent renormalization prescription, like the MS [12] or its popular modification, the $\overline{\mathrm{MS}}$ [13] scheme. These schemes are characterized through the fact that their renormalization group functions are massindependent which makes renormalization group improvements much more transparent. It also has the advantage that the computation of the renormalization group functions themselves is significantly simplified (cf. Section 2). On the other hand there is the big drawback that the decoupling theorem of Appelquist and Carazzone 48 does not hold in mass-independent schemes. This is due to the mass-independence of the renormalization group functions which implies that, e.g. in case of QCD, the top quark and the down quark have identical contributions.

Note that in the broad classes of momentum subtraction schemes the decoupling theorem is valid. However, the calculations are much more complicated and in general a coupled system of differential equations involving also the quark masses and the gauge parameter has to be solved in order to obtain the running of the couplings.

Coming back to our example this means that the cross section $\sigma\left(e^{+} e^{-} \rightarrow b \bar{b}+\right.$ gluons $)$ evaluated in the $\overline{\mathrm{MS}}$ scheme with $\sqrt{s}=40 \mathrm{GeV}$ does not behave like $\sqrt{s} / M_{t}$ but still contains logarithms of the form $\ln \left(s / M_{t}^{2}\right)$, which arise at order $\alpha_{s}^{2}$ from the diagram in Fig. 3.1.

Clearly, both from the theoretical and practical point of view this is not acceptable. The way out is the explicit construction of an effective Lagrangian where the heavy particles are integrated out. This means that the dynamical degree of freedom of the heavy quark is removed, which manifestly leads to power-suppressed contributions of the latter. This will be performed in Section 3.1 for the case of QCD. The effective Lagrangian

\footnotetext{
${ }^{5}$ Actually the proof of the decoupling theorem in 48 is performed for a momentum-subtraction scheme.
} 


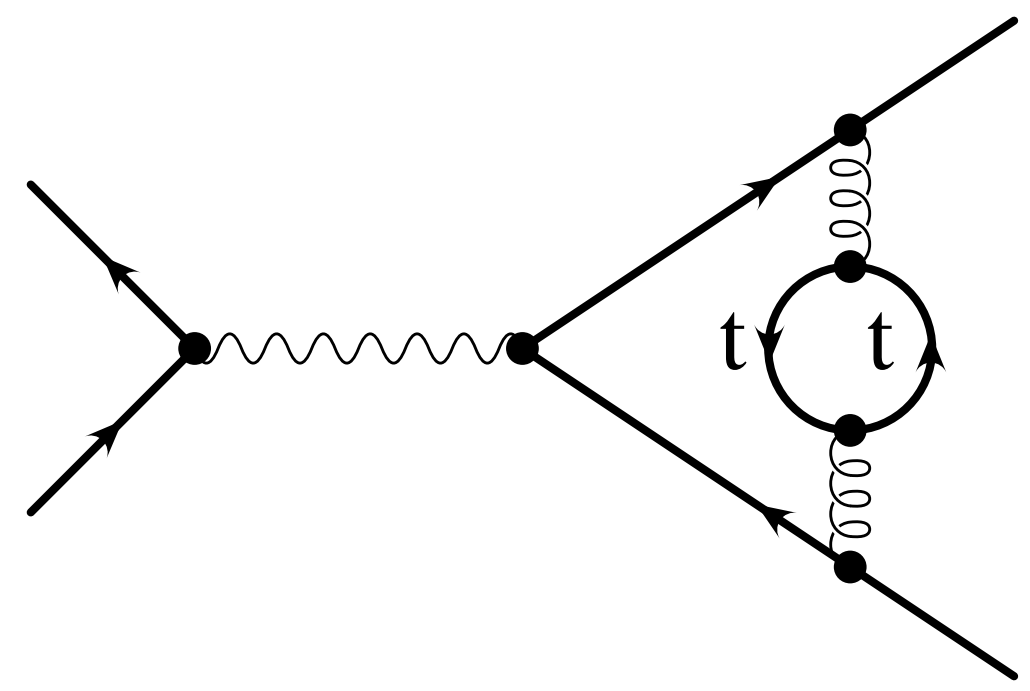

Figure 3.1: Feynman diagram contribution to the process $e^{+} e^{-} \rightarrow b \bar{b}$ which gives rise to top-quark-dependent terms.

only depends on light degrees of freedom where the couplings scaled by the so-called decoupling constants. These constants are universal and have been computed in the case of QCD up to order $\alpha_{s}^{3}$ 49.

Concerning the practical consequences we again want to consider the example of $e^{+} e^{-}$ annihilation. For a calculation at order $\alpha_{s}^{2}$ one computes all relevant diagrams including the one of Fig. 3.1. As mentioned above the result diverges proportional to the logarithm of the heavy quark mass. Now one has to remember that the coupling has to be changed according to the known rules which describe the transition to the effective Lagrangian. Thus one arrives at a physical observable expressed in terms of parameters of the effective Lagrangian and it can be explicitly checked that the dependence on the heavy quark mass, $M_{t}$, is power-suppressed - in the case at hand it goes like $s / M_{t}^{2}$.

Pioneering work in the computation of the decoupling constants has been done in [50]. In Ref. [51] the decoupling constant for $\alpha_{s}$ has been computed at the two-loop order. The crucial idea of the method is based on the fact that the decoupling theorem 48] works in momentum subtraction schemes. Thus after relating the corresponding coupling constant to $\alpha_{s}$ defined in the $\overline{\mathrm{MS}}$ scheme both in the full and the effective theory, it is possible to derive differential equations for the decoupling constant. They can easily be solved. For the corresponding integration constant a two-loop calculation is needed.

Thirteen years later the authors of [52] evaluated the corrections of order $\alpha_{s}^{3}$ to the total decay rate of the $Z$ boson induced by a heavy top quark. In order to make the decoupling explicit the two-loop result of [51] is needed. However, it turned out that after expressing the decay rate in terms of effective parameters the top quark did not decouple.

\footnotetext{
${ }^{6}$ Here we mean "normal" coupling constants, but also masses, gauge parameters, etc..

${ }^{7}$ Note, that, in the case where the light quark masses are neglected, only $\alpha_{s}$ remains as a parameter.
} 
Thus in [52] a second evaluation of the decoupling constant for $\alpha_{s}$ has been performed with a different result as in [51]. The result of [52] was confirmed in [49]. In the meantime, the authors of Ref. 51] have revised [53] their original analysis and have found agreement with Ref. [52].

The method of Ref. 52] for the computation of the decoupling relations is based on the evaluation of a top quark contribution to a physical quantity. In particular the authors of [52] considered corrections to the massless quark propagator with an additional zero momentum operator insertion. In order to obtain the decoupling relations to order $\alpha_{s}^{2}$ a three-loop calculation corresponding to the order $\alpha_{s}^{3}$ corrections is necessary. Correspondingly, the decoupling relation to order $\alpha_{s}^{3}$ would require a four-loop calculation. In our method, which is described below, only the computation of three-loop vacuum diagrams are necessary.

\subsection{Construction of an effective Lagrangian in QCD}

The main idea of effective theories is that the dynamics at low energies does not depend on the details of the dynamics of high energies. Thus the low-energy physics can be described using an effective Lagrangian which does not depend on the additional degrees of freedom present at high energies. The only effect of the high-energy parameters are modified couplings of the effective Lagrangian with respect to the full one.

In the following we want to describe the construction of the effective Lagrangian in QCD with one heavy quark of mass $m_{h}$ and $n_{l}$ light quarks.

Our starting point is the full QCD Lagrangian which reads

$$
\begin{aligned}
\mathcal{L}^{\mathrm{QCD}}= & -\frac{1}{4} G^{a, \mu \nu} G_{\mu \nu}^{a}+\sum_{f=1}^{n_{f}} \bar{\psi}_{f}\left(i \not D-m_{f}\right) \psi_{f} \\
& -\frac{1}{2(1-\xi)}\left(\partial^{\mu} G_{\mu}^{a}\right)^{2}+\partial^{\mu} \bar{c}^{a}\left(\partial_{\mu} c^{a}-g_{s} f^{a b c} c^{b} G_{\mu}^{c}\right),
\end{aligned}
$$

where the field strength tensor is defined through $G^{a, \mu \nu}=\partial^{\mu} G^{a, \nu}-\partial^{\nu} G^{a, \mu}+g_{s} f^{a b c} G^{b, \mu} G^{c, \nu}$. $f^{a b c}$ are the structure constants of the QCD gauge group, $g_{s}=\sqrt{4 \pi \alpha_{s}}$ is the QCD gauge coupling, $\psi_{f}$ is a quark field with mass $m_{f}, G^{a, \mu}$ is the gluon field, $c^{a}$ is the Faddeev-Popovghost field, and $n_{f}=n_{l}+1$ is the total number of quark flavours. $D_{\mu}=\partial_{\mu}-i g_{s}\left(\lambda^{a} / 2\right) G_{\mu}^{a}$ is the covariant derivative in the fundamental representation and $\lambda^{a}$ are the Gell-Mann matrices. For convenience we list the gluon propagator resulting from (3.1)

$$
D_{g}(q)=\frac{i}{q^{2}+i \epsilon}\left(-g^{\mu \nu}+\xi \frac{q^{\mu} q^{\nu}}{q^{2}}\right) .
$$

In this convention $\xi=0$ corresponds to Feynman gauge and $\xi=1$ to Landau gauge.

For later use we define the renormalization constants connecting the bare and renormalized quantities in Eq. (3.1):

$$
\begin{aligned}
& g_{s}^{0}=\mu^{\varepsilon} Z_{g} g_{s}, \quad m_{q}^{0}=Z_{m} m_{q}, \quad \xi^{0}-1=Z_{3}(\xi-1), \\
& \psi_{q}^{0}=\sqrt{Z_{2}} \psi_{q}, \quad G_{\mu}^{0, a}=\sqrt{Z_{3}} G_{\mu}^{a}, \quad c^{0, a}=\sqrt{\tilde{Z}_{3}} c^{a} .
\end{aligned}
$$


In addition one introduces the renormalization constants of the quark-gluon, three-gluon, four-gluon and gluon-ghost vertex which are denoted by $Z_{1 F}, Z_{1}, Z_{4}$ and $\tilde{Z}_{1}$. The SlavnovTaylor identities connecting the different renormalization constants can, e.g., be found in [23].

It is clear that in the effective Lagrangian $\mathcal{L}_{\text {eff }}^{\mathrm{QCD}}$ all explicit trace to the heavy quark must have disappeared. However, the mathematical structure must be identical to the one of $\mathcal{L}^{\mathrm{QCD}}$ in Eq. (3.1). This is because $\mathcal{L}^{\mathrm{QCD}}$ represents the most general Lagrangian describing the interaction of quarks and gluons and respecting the symmetry properties imposed by the Becchi-Rouet-Stora-Tyutin invariance [54]. Of course, this also has to be respected by a $n_{l}$-flavour theory described by $\mathcal{L}_{\text {eff }}^{\mathrm{QCD}}$. Nevertheless the parameters and fields of $\mathcal{L}_{\text {eff }}^{\mathrm{QCD}}$ are different from the ones of the full theory. It is convenient to define the corresponding relations in analogy to the renormalization constants of Eq. (3.3) and introduce multiplicative factors - the so-called decoupling constants $\zeta_{i}^{0}$. Thereby it is advantageous to consider the decoupling relations in the bare theory which is indicated by the superscript zero. The renormalization is performed afterwards. Thus we define

$$
\begin{array}{lll}
g_{s}^{0, \prime}=\zeta_{g}^{0} g_{s}^{0}, & m_{q}^{0, \prime}=\zeta_{m}^{0} m_{q}^{0}, & \xi^{0, \prime}-1=\zeta_{3}^{0}\left(\xi^{0}-1\right), \\
\psi_{q}^{0, \prime}=\sqrt{\zeta_{2}^{0}} \psi_{q}^{0}, & G_{\mu}^{0,,, a}=\sqrt{\zeta_{3}^{0}} G_{\mu}^{0, a}, & c^{0,, a}=\sqrt{\tilde{\zeta}_{3}^{0}} c^{0, a},
\end{array}
$$

where the primes mark the quantities of the effective $n_{l}$-flavour theory.

Taking into account these considerations we can write down a defining equation for the bare effective Lagrangian in terms of the full Lagrangian with re-scaled parameters:

$$
\mathcal{L}_{\text {eff }}^{\mathrm{QCD}}\left(g_{s}^{0}, m_{q}^{0}, \xi^{0} ; \psi_{q}^{0}, G_{\mu}^{0, a}, c^{0, a} ; \zeta_{i}^{0}\right)=\mathcal{L}^{\mathrm{QCD}}\left(g_{s}^{0, \prime}, m_{q}^{0, \prime}, \xi^{0, \prime} ; \psi_{q}^{0, \prime}, G_{\mu}^{0, l, a}, c^{0,,, a}\right),
$$

where $\mathcal{L}^{\mathrm{QCD}}$ is given in Eq. (3.1), $q$ represents the $n_{l}$ light-quark flavours and $\zeta_{i}^{0}$ collectively denotes all bare decoupling constants of Eq. (3.4). Once they are explicitly computed the effective Lagrangian is completely determined. Green functions of light fields obtained from $\mathcal{L}^{\mathrm{QCD}}$ agree with the ones of $\mathcal{L}_{\text {eff }}^{\mathrm{QCD}}$ up to terms suppressed by inverse powers of the heavy quark mass.

In the language of effective theories the computation of the decoupling constants is referred to as matching calculation. It can be performed in a more or less complicated way. As it is even nowadays highly non-trivial to apply the methods of [51] and [52] at order $\alpha_{s}^{3}$, we developed a procedure which relates the $n$-loop decoupling constants of Eq. (3.4) to $n$-loop massive one-scale integrals. It will be described in the next Subsection.

\subsection{Computation of the decoupling constants}

In order to compute the decoupling constants of Eq. (3.4) we have to find convenient Green functions which have to be considered both in the effective and full theory. For this reason we define the bare two-point functions for quarks, gluons and ghosts as follows:

$$
\frac{1}{m-\not p-\Sigma^{0}(p)}=i \int \mathrm{d}^{4} x e^{i p x}\left\langle T \psi_{q}^{0}(x) \bar{\psi}_{q}^{0}(0)\right\rangle
$$




$$
\begin{aligned}
\frac{\delta^{a b}\left(-g_{\mu \nu}+\frac{p^{\mu} p^{\nu}}{p^{2}}\right)}{-p^{2}\left(1+\Pi_{G}^{0}\left(p^{2}\right)\right)}+\ldots & =i \int \mathrm{d}^{4} x e^{i p x}\left\langle T G_{\mu}^{0, a}(x) G_{\nu}^{0, b}(0)\right\rangle \\
\frac{\delta^{a b}}{-p^{2}\left(1+\Pi_{c}^{0}\left(p^{2}\right)\right)} & =i \int \mathrm{d}^{4} x e^{i p x}\left\langle T c^{0, a}(x) \bar{c}^{0, b}(0)\right\rangle .
\end{aligned}
$$

The ellipses in the case of the gluon propagator indicate the longitudinal part which we are not interested in.

From the two-point functions of Eqs. (3.6) we will be able to obtain $\zeta_{2}^{0}, \zeta_{m}^{0}, \zeta_{3}^{0}$ and $\tilde{\zeta}_{3}^{0}$. In order to get a relation involving $\zeta_{g}^{0}$ one has at least to consider three-point functions where the coupling $g_{s}$ already appears at Born level. As the vertex between the gluon and ghost is the least complex one we will take it for the computation. In amputated form it is defined through

$$
p^{\mu} g_{s}^{0}\left\{-i f^{a b c}\left[1+\Gamma_{G \bar{c} c}^{0}(p, k)\right]\right\}+\ldots=i^{2} \int \mathrm{d} x \mathrm{~d} y \mathrm{e}^{i(p \cdot x+k \cdot y)}\left\langle T c^{0, a}(x) \bar{c}^{0, b}(0) G^{0, c, \mu}(y)\right\rangle^{1 \mathrm{PI}}
$$

where $p$ and $k$ are the outgoing four-momenta of $c$ and $G$, respectively. The ellipses indicate other colour structures we are not interested in. Note that we pull out a factor $g_{s}^{0}$ on the left-hand side of Eq. (3.7) as it is already present at Born level.

Let us start with the decoupling constant for the gluon field. It is obvious that the gluon propagator constitutes a good candidate to compute $\zeta_{3}^{0}$. Up to terms of order $1 / m_{h}$ we have the following chain of equations

$$
\begin{aligned}
\frac{\delta^{a b}\left(-g_{\mu \nu}+\frac{p^{\mu} p^{\nu}}{p^{2}}\right)}{-p^{2}\left(1+\Pi_{G}^{0}\left(p^{2}\right)\right)} & =i \int \mathrm{d}^{4} x e^{i p x}\left\langle T G_{\mu}^{0, a}(x) G_{\nu}^{0, b}(0)\right\rangle \\
& =\frac{1}{\zeta_{3}^{0}} i \int \mathrm{d}^{4} x e^{i p x}\left\langle T G_{\mu}^{0,,, a}(x) G_{\nu}^{0,,, b}(0)\right\rangle \\
& =\frac{1}{\zeta_{3}^{0}} \frac{\delta^{a b}\left(-g_{\mu \nu}+\frac{p^{\mu} p^{\nu}}{p^{2}}\right)}{-p^{2}\left(1+\Pi_{G}^{0 \prime}\left(p^{2}\right)\right)}
\end{aligned}
$$

where in the second step Eqs. (3.4) has been used. Note that $\Pi_{G}^{0 \prime}\left(p^{2}\right)$ only contains light degrees of freedom whereas $\Pi_{G}^{0}\left(p^{2}\right)$ also receives virtual contributions from the heavy quark $h$. Eq. (3.8) provides a formulae for $\zeta_{3}^{0}$

$$
\zeta_{3}^{0}=\frac{1+\Pi_{G}^{0}\left(p^{2}\right)}{1+\Pi_{G}^{0 \prime}\left(p^{2}\right)} .
$$

From the construction of the effective theory it is clear that the decoupling constants do not depend on the momentum transfer. Thus also the right-hand side of (3.9) has to be independent of the external momentum $p$. This means that we can choose any convenient momentum for the computation. In particular it is possible to choose $p=0$. This has the advantage that only vacuum diagrams have to be considered. Since we work in the framework of dimensional regularization all scaleless integrals can be set to zero. As a 
consequence we have $\Pi_{G}^{0 \prime}(0)=0$ and the contribution to $\Pi_{G}^{0}$ is given by the diagrams containing at least one heavy quark line. In the following we attach to the corresponding contributions an additional index "h" and refer to it as the "hard part". Finally we arrive at the compact formula

$$
\zeta_{3}^{0}=1+\Pi_{G}^{0, h}(0)
$$

The $n$-loop contribution to $\zeta_{3}^{0}$ is related to the $n$-loop vacuum diagrams where the scale is given by the mass of the heavy quark. At one-loop order only one diagram contributes to $\zeta_{3}^{0}$. At two loops there are already three diagrams and at three-loop order altogether 189 diagrams have to be taken into account. A typical example is pictured in Fig. 3.3. We want to mention that the bare decoupling constants may contain non-local terms like $\ln \left(\mu^{2} / m_{h}^{2}\right) / \varepsilon$, which is in contrast to the renormalization constants.

After the computation of the bare diagrams the parameters (in our case $\alpha_{s}, \xi$ and the heavy quark mass, $m_{h}$ ) have to be expressed in terms of their renormalized counterparts. The finite decoupling constant is obtained from Eq. (3.4) after expressing the bare fields in terms of the renormalized ones via Eq. (3.3)

$$
G_{\mu}^{\prime}=\sqrt{\frac{Z_{3} \zeta_{3}^{0}}{Z_{3}^{\prime}}} G_{\mu}=\sqrt{\zeta_{3}} G_{\mu} .
$$

Note that $Z_{3}^{\prime}$ depends on $\alpha_{s}^{\prime}$ and $\xi^{\prime}$. They have to be transformed to $\alpha_{s}$ and $\xi$ with the help of $\zeta_{g}$ and $\zeta_{3}$, respectively, which are needed up to $(l-1)$-loop accuracy if Eq. (3.11) is considered at $l$-loop order.

The ghost propagator can be treated in complete analogy and one arrives at the following formula for the bare decoupling constant for the ghost field

$$
\tilde{\zeta}_{3}^{0}=1+\Pi_{c}^{0, h}(0)
$$

There is no diagram which contributes at one-loop order and one at order $\alpha_{s}^{2}$. Also at three-loop order the number of diagrams is moderate and amounts to 25.

The renormalized version of Eq. (3.12) reads

$$
\tilde{\zeta}_{3}=\frac{\tilde{Z}_{3} \tilde{\zeta}_{3}^{0}}{\tilde{Z}_{3}^{\prime}} .
$$

In order to obtain expressions for $\zeta_{2}$ and $\zeta_{m}$ one considers the light-quark propagator which leads to the following chain of relations:

$$
\begin{aligned}
\frac{1}{m-\not p-\Sigma(p)} & =\frac{1}{m\left(1-\Sigma_{S}^{0}\left(p^{2}\right)\right)-\not p\left(1+\Sigma_{V}^{0}\left(p^{2}\right)\right)} \\
& =\frac{1}{\zeta_{2}^{0} \zeta_{m}^{0} m\left(1-\Sigma_{S}^{0 \prime}\left(p^{2}\right)\right)-\zeta_{2}^{0} \not p\left(1+\Sigma_{V}^{0 \prime}\left(p^{2}\right)\right)}
\end{aligned}
$$


Nullifying the external momentum $p$ in the self energies leads to

$$
\begin{aligned}
\zeta_{2}^{0} & =1+\Sigma_{V}^{0, h}(0), \\
\zeta_{m}^{0} & =\frac{1-\Sigma_{S}^{0, h}(0)}{1+\Sigma_{V}^{0, h}(0)},
\end{aligned}
$$

and the finite expressions are obtained from ${ }^{8}$

$$
\begin{aligned}
\zeta_{2} & =\frac{Z_{2} \zeta_{2}^{0}}{Z_{2}^{\prime}}, \\
\zeta_{m} & =\frac{Z_{m} \zeta_{m}^{0}}{Z_{m}^{\prime}} .
\end{aligned}
$$

Similarly to $\Pi_{c}^{0, h}(0)$ there are no one-loop diagrams contributing to $\Sigma_{V}^{0, h}(0)$ and $\Sigma_{S}^{0, h}(0)$ and at two- and three-loop order again one and 25 diagrams, respectively, have to be considered. Typical specimen are depicted in Fig. 3.2. Actually, through three loops, Eq. (3.18) simplifies to $\zeta_{m}^{0}=1-\Sigma_{V}^{0, h}(0)-\Sigma_{S}^{0, h}(0)$. It should be noted that the vector and scalar parts separately still depend on the QCD gauge parameter $\xi$, but $\xi$ drops out in their sum, which is a useful check for our calculation.
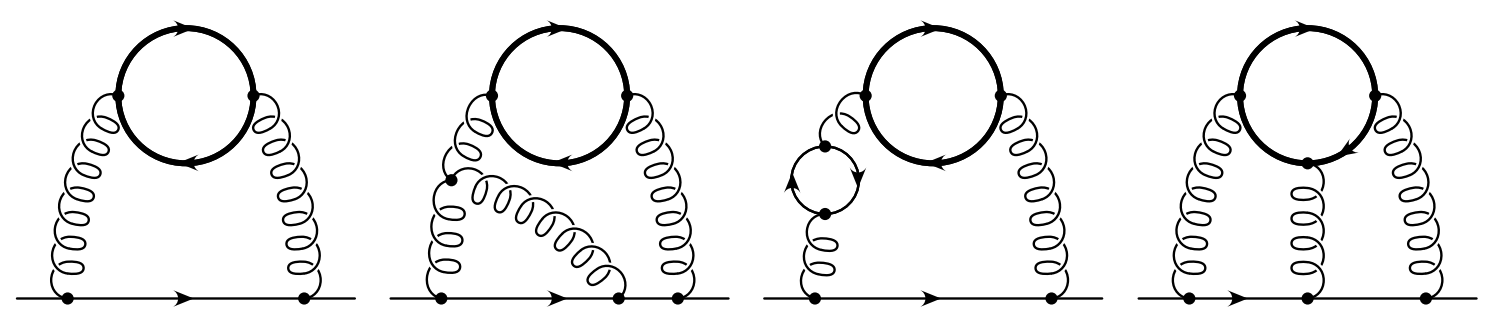

Figure 3.2: Typical three-loop diagrams pertinent to $\Sigma_{V}^{0, h}(0)$ and $\Sigma_{S}^{0, h}(0)$. Solid, boldfaced, and loopy lines represent massless quarks $q$, heavy quarks $h$, and gluons $G$, respectively.

The derivation of a formula for $\zeta_{g}^{0}$ is slightly more involved. As a starting point we choose the full, i.e. non-amputated gluon-ghost Green function and get (using Eqs. (3.4)) the following equations

$$
\begin{aligned}
g_{s}^{0} G_{G \bar{c} c}^{\mu, a b c}(p, k) & =i^{2} \int \mathrm{d} x \mathrm{~d} y \mathrm{e}^{i(p \cdot x+k \cdot y)}\left\langle T c^{0, a}(x) \bar{c}^{0, b}(0) G^{0, c, \mu}(y)\right\rangle \\
& =\frac{1}{\tilde{\zeta}_{3}^{0} \sqrt{\zeta_{3}^{0}}} i^{2} \int \mathrm{d} x \mathrm{~d} y \mathrm{e}^{i(p \cdot x+k \cdot y)}\left\langle T c^{0, a}(x) \bar{c}^{0, b}(0) G^{0, c, \mu}(y)\right\rangle^{\prime} \\
& =\frac{1}{\tilde{\zeta}_{3}^{0} \sqrt{\zeta_{3}^{0}}} g_{s}^{0, \prime} G_{G \bar{c} c c}^{\mu, a b c,}(p, k)
\end{aligned}
$$

\footnotetext{
${ }^{8}$ Note that the same symbol is also used for Riemann's zeta function $\zeta_{2}=\pi^{2} / 6$. However, as they appear in a completely different context confusion is not possible.
} 


$$
=\frac{\zeta_{g}^{0}}{\tilde{\zeta}_{3}^{0} \sqrt{\zeta_{3}^{0}}} g_{s}^{0} G_{G \bar{c} c}^{\mu, a b c, \prime}(p, k)
$$

where $p$ and $k$ are the outgoing four-momenta of $c$ and $G$, respectively. At this point we should amputate the Green functions by multiplying with the inverse propagators of the external gluon and ghost fields in the full theory. From the decoupling relations derived above we get an additional factor $\left(\tilde{\zeta}_{3}^{0}\right)^{2} \zeta_{3}^{0}$ on the right-hand side of Eq. (3.19) which leads to

$$
\left[1+\Gamma_{G \bar{c} c}^{0}(p, k)\right]=\zeta_{g}^{0} \tilde{\zeta}_{3}^{0} \sqrt{\zeta_{3}^{0}}\left[1+\Gamma_{G \bar{c} c}^{0 \prime}(p, k)\right] .
$$

Here, $\Gamma_{G \bar{c} c}^{0}(p, k)$ is defined through the 1PI part of the amputated gluon-ghost Green function as introduced in Eq. (3.7). Setting to zero the external momenta we obtain

$$
\zeta_{g}^{0}=\frac{\tilde{\zeta}_{1}^{0}}{\tilde{\zeta}_{3}^{0} \sqrt{\zeta_{3}^{0}}},
$$

where

$$
\tilde{\zeta}_{1}^{0}=1+\Gamma_{G \bar{c} c}^{0, h}(0,0)
$$

is the decoupling constant belonging to the gluon-ghost vertex. Again, the renormalized version of the decoupling constant is obtained with the help of the renormalization constants in the full and effective theory as

$$
\zeta_{g}=\frac{Z_{g} \zeta_{g}^{0}}{Z_{g}^{\prime}} .
$$

Thus, in order to compute $\zeta_{g}$ one has to evaluate the decoupling constant for the gluon propagator, the ghost propagator and the gluon-ghost vertex. We could have chosen also another vertex involving the strong coupling, e.g. the quark-gluon or the threegluon vertex, and would have arrived at a similar expression as in Eq. (3.21). This is in complete analogy to the renormalization constants where due to Slavnov-Taylor identities the various renormalization constants are related to each other (see, e.g., Ref. [23]).

We should mention that $\Gamma_{G \bar{c} c}^{0, h}(0,0)$ has no one-loop contribution and there are five diagrams at two loops, which, however, add up to zero. A non-zero contribution is obtained at order $\alpha_{s}^{3}$ where 228 diagrams contribute. A typical representative is shown in Fig. 3.3.

At order $\alpha_{s}^{2}$ the three contributions to $\zeta_{g}^{0}$ are still separately independent of the gauge parameter $\xi$, so that the $\xi$ independence of their combination does not provide a meaningful check for our calculation. The situation changes at $\mathcal{O}\left(\alpha_{s}^{3}\right)$, where all three parts separately depend on $\xi$ and only their proper combination is $\xi$ independent as is required for a physical quantity. In the calculation this has been used as a check.

In Eqs. (3.10), (3.12), (3.15), (3.16) and (3.21) the bare decoupling constants $\zeta_{3}^{0}, \tilde{\zeta}_{3}^{0}$, $\zeta_{2}^{0}, \zeta_{m}^{0}$ and $\zeta_{g}^{0}$ are expressed in terms of vacuum diagrams. Thus, if the former are to 

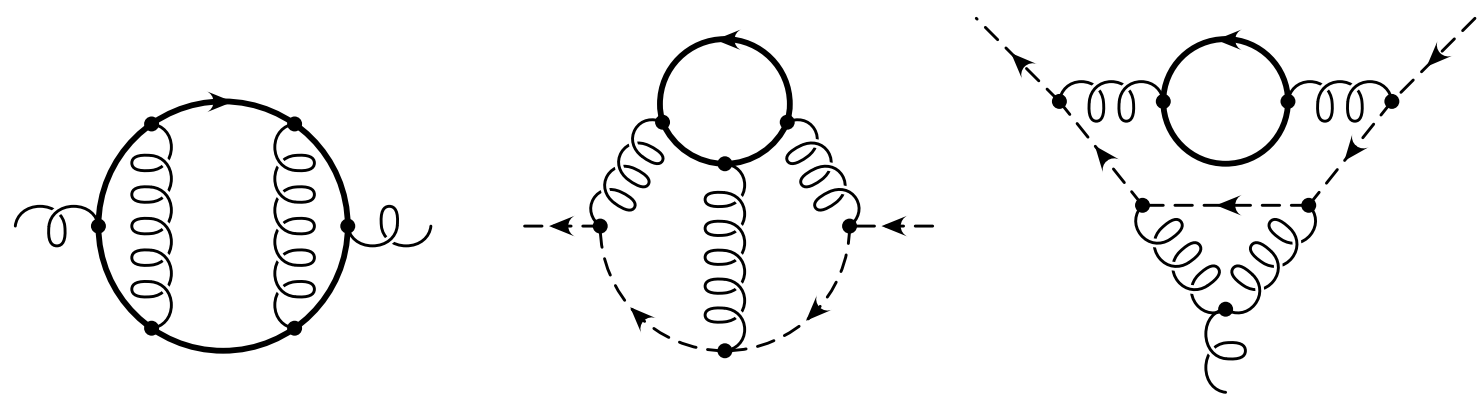

Figure 3.3: Typical three-loop diagrams pertinent to $\Pi_{G}^{0, h}(0), \Pi_{c}^{0, h}(0)$, and $\Gamma_{G \bar{c} c}^{0, h}(0,0)$. Bold-faced, loopy, and dashed lines represent heavy quarks $h$, gluons $G$, and FaddeevPopov ghosts $c$, respectively.

be computed at order $\alpha_{s}^{3}$ the latter need to be known at three-loop order. In the recent years three-loop vacuum diagrams have been studied extensively and a FORM [55] package, MATAD [35], has been written which allows for an automated computation.

At this point we would like to make a comment on the different kind of poles which appear in the calculation. If we choose zero external momentum there are in general both UV and IR poles in the individual diagrams. However, the renormalization constants in Eq. (3.3) only contain UV divergences. Thus the two different kind of poles have to be identified in order to arrive at a finite expression for the decoupling constants. This is a special feature of dimensional regularization which allows to treat simultaneously UV and IR divergences and to set to zero scaleless integrals. More details to this context can be found in Ref. [56] and references cited therein.

\subsection{Results}

In the following we list the analytical results for the renormalized decoupling constants $\zeta_{g}$ and $\zeta_{m}$, which relate the physical parameters in the full theory to their counterparts in the effective theory. In Appendix B we also provide the (gauge parameter dependent) results for $\zeta_{2}$ and $\zeta_{3}$.

As already mentioned above, the computation can be reduced to one-scale vacuum integrals (see also Appendix A.2). However, the large number of diagrams requires the automation of the computation. Actually the computation of $\zeta_{g}$ was one of the first application of the package GEFICOM [41] (cf. Appendix A.3), which combines several stand-alone program packages in order to automate the computation from the generation of the diagrams to the summation of the individual results. For the evaluation of the decoupling constants all diagrams have been generated automatically with the Fortran program QGRAF [57] and the integrations have been performed using the FORM [55] package MATAD [35].

Note, that in contrast to the renormalization constants $Z_{i}$ in Eq. (3.3), the decoupling constants $\zeta_{i}^{0}$ also receive contributions from the finite parts of the loop integrals. Thus, at 
$\mathcal{O}\left(\alpha_{s}^{3}\right)$, we are led to evaluate three-loop tadpole integrals also retaining their finite parts.

For illustration of the formalism derived in the previous Subsection we want to compute the lowest-order contribution to $\zeta_{2}$ which is of $\mathcal{O}\left(\alpha_{s}^{2}\right)$ and comes from the first diagram in Fig. 3.2. According to Eq. (3.15) the vector part has to be evaluated for zero external momentum. This is conveniently done with the help of

$$
\Sigma_{V}^{0, h}(0)=\left.\frac{1}{4 p^{2}} \operatorname{Tr}\left[\not p \Sigma^{0, h}(p)\right]\right|_{p=0},
$$

where a simple Taylor expansion up to linear order has to be performed for $\Sigma^{0, h}(p)$. As only vacuum integrals are involved in the computation one can easily perform the tensor reduction in the numerator and then use Eq. (A.7) in order to arrive at the result for the bare decoupling constant

$$
\zeta_{2}^{0}=1+\left(\frac{\alpha_{s}}{\pi}\right)^{2} C_{F} T\left[-\frac{1}{16 \varepsilon}+\frac{5}{96}-\frac{1}{8} \ln \frac{\mu^{2}}{m_{h}^{2}}+\mathcal{O}(\varepsilon)\right] .
$$

Now Eq. (3.17) can be used to get a finite result. Therefore the ratio $Z_{2} / Z_{2}^{\prime}$ is needed to order $\alpha_{s}^{2}$ where the parameters $\alpha_{s}^{\prime}$ and $\xi^{\prime}$ in $Z_{2}^{\prime}$ have to be expressed in terms of $\alpha_{s}$ and $\xi$. with the help of Eqs. (3.4). Actually, to our order the contributions from $\zeta_{g}$ and $\zeta_{3}$ exactly cancel and we are left with

$$
\frac{Z_{2}}{Z_{2}^{\prime}}=1+\left(\frac{\alpha_{s}}{\pi}\right)^{2} \frac{C_{F} T}{16 \varepsilon} .
$$

Inserting Eqs. (3.25) and (3.26) in (3.17) finally leads to

$$
\zeta_{2}^{\mathrm{MS}}=1+\left(\frac{\alpha_{s}}{\pi}\right)^{2} C_{F} T\left[\frac{5}{96}-\frac{1}{8} \ln \frac{\mu^{2}}{m_{h}^{2}}\right],
$$

which agrees with Eq. (B.5).

In the same way also the scalar part of the quark self energy can be treated in order to obain with the help of Eq. (3.16) a result for $\zeta_{m}$. Taking also the three-loop diagrams into account we obtain

$$
\begin{aligned}
\zeta_{m}^{\mathrm{OS}}= & +\left(\frac{\alpha_{s}^{\left(n_{f}\right)}(\mu)}{\pi}\right)^{2}\left(\frac{89}{432}-\frac{5}{36} \ln \frac{\mu^{2}}{M_{h}^{2}}+\frac{1}{12} \ln ^{2} \frac{\mu^{2}}{M_{h}^{2}}\right)+\left(\frac{\alpha_{s}^{\left(n_{f}\right)}(\mu)}{\pi}\right)^{3}\left[\frac{1871}{2916}\right. \\
& -\frac{407}{864} \zeta_{3}+\frac{5}{4} \zeta_{4}-\frac{1}{36} B_{4}+\left(\frac{121}{2592}-\frac{5}{6} \zeta_{3}\right) \ln \frac{\mu^{2}}{M_{h}^{2}}+\frac{319}{432} \ln ^{2} \frac{\mu^{2}}{M_{h}^{2}} \\
& \left.+\frac{29}{216} \ln ^{3} \frac{\mu^{2}}{M_{h}^{2}}+n_{l}\left(\frac{1327}{11664}-\frac{2}{27} \zeta_{3}-\frac{53}{432} \ln \frac{\mu^{2}}{M_{h}^{2}}-\frac{1}{108} \ln ^{3} \frac{\mu^{2}}{M_{h}^{2}}\right)\right] \\
\approx & 1+0.2060\left(\frac{\alpha_{s}^{\left(n_{f}\right)}\left(M_{h}\right)}{\pi}\right)^{2}+\left(1.4773+0.0247 n_{l}\right)\left(\frac{\alpha_{s}^{\left(n_{f}\right)}\left(M_{h}\right)}{\pi}\right)^{3} .
\end{aligned}
$$


where $\zeta_{3} \approx 1.202057$ and

$$
\begin{aligned}
B_{4} & =16 \operatorname{Li}_{4}\left(\frac{1}{2}\right)-\frac{13}{2} \zeta_{4}-4 \zeta_{2} \ln ^{2} 2+\frac{2}{3} \ln ^{4} 2 \\
& \approx-1.762800
\end{aligned}
$$

with $\mathrm{Li}_{4}$ being the quadrilogarithm, is a constant typical for three-loop vacuum diagrams [58]. $n_{l}=n_{f}-1$ is the number of light-quark flavours, and $M_{h}$ is the on-shell mass of the heavy quark $h$. For the numerical evaluation in the last line of Eq. (3.28), we have chosen $\mu=M_{h}$. The $\mathcal{O}\left(\alpha_{s}^{2}\right)$ term of Eq. (3.28) is computed in Ref. [51] and the $\mathcal{O}\left(\alpha_{s}^{3}\right)$ term can be found in 49 .

The proper combination of the three ingredients entering the calculation of $\zeta_{g}^{0}$, namely the hard parts of the gluon and ghost propagators and the gluon-ghost vertex correction, lead to

$$
\begin{aligned}
\left(\zeta_{g}^{\mathrm{OS}}\right)^{2}= & +\frac{\alpha_{s}^{\left(n_{f}\right)}(\mu)}{\pi}\left(-\frac{1}{6} \ln \frac{\mu^{2}}{M_{h}^{2}}\right)+\left(\frac{\alpha_{s}^{\left(n_{f}\right)}(\mu)}{\pi}\right)^{2}\left(-\frac{7}{24}-\frac{19}{24} \ln \frac{\mu^{2}}{M_{h}^{2}}+\frac{1}{36} \ln ^{2} \frac{\mu^{2}}{M_{h}^{2}}\right) \\
& +\left(\frac{\alpha_{s}^{\left(n_{f}\right)}(\mu)}{\pi}\right)^{3}\left[-\frac{58933}{124416}-\frac{2}{3} \zeta_{2}\left(1+\frac{1}{3} \ln 2\right)-\frac{80507}{27648} \zeta_{3}-\frac{8521}{1728} \ln \frac{\mu^{2}}{M_{h}^{2}}\right. \\
& \left.-\frac{131}{576} \ln ^{2} \frac{\mu^{2}}{M_{h}^{2}}-\frac{1}{216} \ln ^{3} \frac{\mu^{2}}{M_{h}^{2}}+n_{l}\left(\frac{2479}{31104}+\frac{\zeta_{2}}{9}+\frac{409}{1728} \ln \frac{\mu^{2}}{M_{h}^{2}}\right)\right] \\
\approx & 1-0.2917\left(\frac{\alpha_{s}^{\left(n_{f}\right)}\left(M_{h}\right)}{\pi}\right)^{2}+\left(-5.3239+0.2625 n_{l}\right)\left(\frac{\alpha_{s}^{\left(n_{f}\right)}\left(M_{h}\right)}{\pi}\right)^{3} .
\end{aligned}
$$

The $\mathcal{O}\left(\alpha_{s}^{3}\right)$ term in Eq. (3.30) is published 49. Leaving aside this term, the results in Eq. (3.30) can be found in Refs. [51, 52, 53].

Notice that the $\mathcal{O}\left(\alpha_{s}^{3}\right)$ terms of $\zeta_{m}$ and $\zeta_{g}$ depend on the number $n_{l}$ of light (massless) quark flavours. However, this dependence is feeble.

The generalization of Eqs. (3.28) and (3.30), appropriate for the gauge group $\mathrm{SU}\left(N_{c}\right)$, is listed in Appendix $\mathrm{B}$. There, also the results expressed in terms of the $\overline{\mathrm{MS}}$ quark mass can be found. In Ref. [59 the leading Yukawa corrections proportional to $G_{F} M_{t}^{2}$ have been computed for $\zeta_{m}$ and $\zeta_{g}$. In Appendix $\mathrm{B}$ also these results are listed.

\subsection{Applications}

\section{Cross section $\sigma\left(e^{+} e^{-} \rightarrow b \bar{b}\right)$}

At this point we would like to pick up the example mentioned at the beginning of this section, namely the $\mathcal{O}\left(\alpha_{s}^{2}\right)$ corrections to the cross section $e^{+} e^{-} \rightarrow b \bar{b}$. If we consider 
center-of-mass energies where the first five quarks can be neglected one obtains?

$$
\begin{aligned}
R(s)= & 3 Q_{b}^{2}\left\{1+\frac{\alpha_{s}^{(6)}(\mu)}{\pi}+\left(\frac{\alpha_{s}^{(6)}(\mu)}{\pi}\right)^{2}\left[c_{2}-\frac{1}{6} \ln \frac{\mu^{2}}{M_{t}^{2}}+\frac{s}{4 M_{t}^{2}}\left(\frac{176}{675}-\frac{8}{135} \ln \frac{s}{M_{t}^{2}}\right)\right.\right. \\
& \left.\left.+\mathcal{O}\left(\frac{s^{2}}{M_{t}^{4}}\right)\right]\right\}+\mathcal{O}\left(\alpha_{s}^{3}\right),
\end{aligned}
$$

where $N_{c}=3$ has been chosen and at order $\alpha_{s}^{2}$ only the contribution from the diagram in Fig. 3.1 is displayed explicitly. All other diagrams are summed in the constant $c_{2}$ [60]. In Eq. (3.31) the definition of the coupling constant still includes the top quark as indicated by the superscript "(6)". Otherwise the computation of the diagram in Fig. 3.1 would hardly be possible. One recognizes that for $M_{t} \rightarrow \infty$ the contribution from the top quark raises logarithmically. Note that the choice $\mu=M_{t}$ does not help as it introduces $\ln M_{t}$ terms in $c_{2}$. At this point Eqs. (3.4) and (3.30) can be used to replace $\alpha_{s}^{(6)}$ in favour of $\alpha_{s}^{(5)}$ which leads to

$$
\begin{aligned}
R(s)= & 3 Q_{b}^{2}\left\{1+\frac{\alpha_{s}^{(5)}(\mu)}{\pi}+\left(\frac{\alpha_{s}^{(5)}(\mu)}{\pi}\right)^{2}\left[c_{2}+\frac{s}{4 M_{t}^{2}}\left(\frac{176}{675}-\frac{8}{135} \ln \frac{s}{M_{t}^{2}}\right)\right.\right. \\
& \left.\left.+\mathcal{O}\left(\frac{s^{2}}{M_{t}^{4}}\right)\right]\right\}+\mathcal{O}\left(\alpha_{s}^{3}\right) .
\end{aligned}
$$

Now the top quark is decoupled, i.e. its contribution goes to zero for $M_{t} \rightarrow \infty$ as $R(s)$ is expressed in terms of $\alpha_{s}^{(5)}$ which is the parameter of the effective theory.

Determination of $\alpha_{s}^{(5)}\left(M_{Z}\right)$ and $m_{q}^{(5)}\left(M_{Z}\right)$ from measurements at the $\tau$ mass scale In the previous example the decoupling relation was only needed to one-loop order. However, the three-loop terms will be indispensable in order to relate the QCD predictions for different observables at next-to-next-to-next-to-leading order. Meaningful estimates of such corrections already exist 61, 62.

Once the corrections of order $\alpha_{s}^{4}$ for $R(s)$ are known they can be used to determine $\alpha_{s}^{(5)}\left(M_{Z}\right)$ from the knowledge of $\alpha_{s}^{(4)}\left(M_{\tau}\right)$ and compare it with other measurements. One would use $R(s)$ to extract四 $\alpha_{s}^{(4)}\left(M_{\tau}\right)$ with an accuracy of order $\alpha_{s}^{4}$ from the data. Then one would use the four-loop $\beta$ function [14] in order to perform the running to the bottomquark threshold. There the three-loop matching relations would be necessary for a consistent decoupling. Using again four-loop running finally leads to $\alpha_{s}^{(5)}\left(M_{Z}\right)$. In the following we will illustrate this procedure. However, instead of determining $\alpha_{s}^{(4)}\left(M_{\tau}\right)$ via $R(s)$ we directly assume a value for $\alpha_{s}^{(4)}\left(M_{\tau}\right)$ and evaluate $\alpha_{s}^{(5)}\left(M_{Z}\right)$ for different choices of the matching scale $\mu^{(5)}$. For the three-loop evolution in connection with two-loop matching

\footnotetext{
${ }^{9}$ Note that this result can immediately be obtained from the example considered in Appendix A.1, Eq. A.3).

${ }^{10}$ The described procedure can also be applied to $\alpha_{s}^{(3)}\left(M_{\tau}\right)$. Only for simplicity and transparency we have chosen $\alpha_{s}^{(4)}\left(M_{\tau}\right)$.
} 
this has been done in Ref. [63]. We are in a position to explore the situation at the next order. It is instructive to include in the analysis also the tree-level and one-loop matching.

Going to higher orders, one expects, on general grounds, that the relation between $\alpha_{s}^{\left(n_{f}-1\right)}\left(\mu^{\prime}\right)$ and $\alpha_{s}^{\left(n_{f}\right)}(\mu)$, where $\mu^{\prime} \ll \mu^{\left(n_{f}\right)} \ll \mu$, becomes insensitive to the choice of the matching scale, $\mu^{\left(n_{f}\right)}$, as long as $\mu^{\left(n_{f}\right)}=\mathcal{O}\left(m_{h}\right)$. In the above-mentioned situation we consider the crossing of the bottom-quark threshold. In particular, we study how the $\mu^{(5)}$ dependence of the relation between $\alpha_{s}^{(4)}\left(M_{\tau}\right)$ and $\alpha_{s}^{(5)}\left(M_{Z}\right)$ is reduced as we implement four-loop evolution with three-loop matching. Our procedure is as follows. We first calculate $\alpha_{s}^{(4)}\left(\mu^{(5)}\right)$ by exactly integrating Eq. (2.35) with the initial condition $\alpha_{s}^{(4)}\left(M_{\tau}\right)=0.36$, then obtain $\alpha_{s}^{(5)}\left(\mu^{(5)}\right)$ from Eqs. (3.4) and (3.30) with $M_{b}=4.7 \mathrm{GeV}$, and finally compute $\alpha_{s}^{(5)}\left(M_{Z}\right)$ with Eq. (2.35). For consistency, $N$-loop evolution must be accompanied by $(N-1)$-loop matching, i.e. if we omit terms of $\mathcal{O}\left(\alpha_{s}^{N+2}\right)$ on the righthand side of Eq. (2.35), we need to discard those of $\mathcal{O}\left(\alpha_{s}^{N}\right)$ in Eq. (3.30) at the same time. In Fig. 3.4, the variation of $\alpha_{s}^{(5)}\left(M_{Z}\right)$ with $\mu^{(5)} / M_{b}$ is displayed for the various levels of accuracy, ranging from one-loop to four-loop evolution. For illustration, $\mu^{(5)}$ is varied rather extremely, by almost two orders of magnitude. While the leading-order result exhibits a strong logarithmic behaviour, the analysis is gradually getting more stable as we go to higher orders. The four-loop curve is almost flat for $\mu^{(5)} \gtrsim 1 \mathrm{GeV}$. Besides the $\mu^{(5)}$ dependence of $\alpha_{s}^{(5)}\left(M_{Z}\right)$, also its absolute normalization is significantly affected by the higher orders. At the central matching scale $\mu^{(5)}=M_{b}$, we encounter a rapid, monotonic convergence behaviour.

Similar analyses may be performed for the light-quark masses as well. For illustration, let us investigate how the $\mu^{(5)}$ dependence of the relation between $\mu_{c}=m_{c}^{(4)}\left(\mu_{c}\right)$ and $m_{c}^{(5)}\left(M_{Z}\right)$ changes under the inclusion of higher orders in evolution and matching. As typical input parameters, we choose $\mu_{c}=1.2 \mathrm{GeV}, M_{b}=4.7 \mathrm{GeV}$, and $\alpha_{s}^{(5)}\left(M_{Z}\right)=0.118$. We first evolve $m_{c}^{(4)}(\mu)$ from $\mu=\mu_{c}$ to $\mu=\mu^{(5)}$ with the help of Eq. (2.12), then obtain $m_{c}^{(5)}\left(\mu^{(5)}\right)$ from Eqs. (3.4) and (3.28), and finally evolve $m_{c}^{(5)}(\mu)$ from $\mu=\mu^{(5)}$ to $\mu=M_{Z}$ using Eq. (2.12). In all steps, $\alpha_{s}^{\left(n_{f}\right)}(\mu)$ is evaluated with the same values of $n_{f}$ and $\mu$ as $m_{c}^{\left(n_{f}\right)}(\mu)$. In Fig. 3.5, we show the resulting values of $m_{c}^{(5)}\left(M_{Z}\right)$ corresponding to $N$-loop evolution with $(N-1)$-loop matching for $N=1, \ldots, 4$. Similarly to Fig. 3.4, we observe a rapid, monotonic convergence behaviour at the central matching scale $\mu^{(5)}=M_{b}$. Again, the prediction for $N=4$ is remarkably stable under the variation of $\mu^{(5)}$ as long as $\mu^{(5)} \gtrsim 1 \mathrm{GeV}$.

The various formulae describing the running and the decoupling of $\alpha_{s}$ and the quark masses are implemented in the program package RunDec [64]. It is realized in Mathematica and provides a convenient possibility to check, e.g., the figures of this Subsection and eventually update the numerical input values. In particular, Fig. 3.4 can easily be reproduced with the help of the procedure AlL2AlH []. After loading RunDec the command AlL2AlH $[0.36,1.777,\{\{5,4.7, \mathrm{mu} 5\}\}, 91.187,1]$ provides the result for $\alpha_{s}^{(5)}\left(M_{Z}\right)$ where the matching has been performed at the scale mu5. $l=1,2,3,4$ corresponds to the number of loops used for the evolution and the values $\alpha_{s}^{(4)}\left(M_{\tau}\right)=0.36, M_{\tau}=1.777 \mathrm{GeV}$, $M_{b}=4.7 \mathrm{GeV}$ and $M_{Z}=91.187 \mathrm{GeV}$ have been adopted. 
$\alpha_{s}^{(5)}\left(M_{Z}\right)$

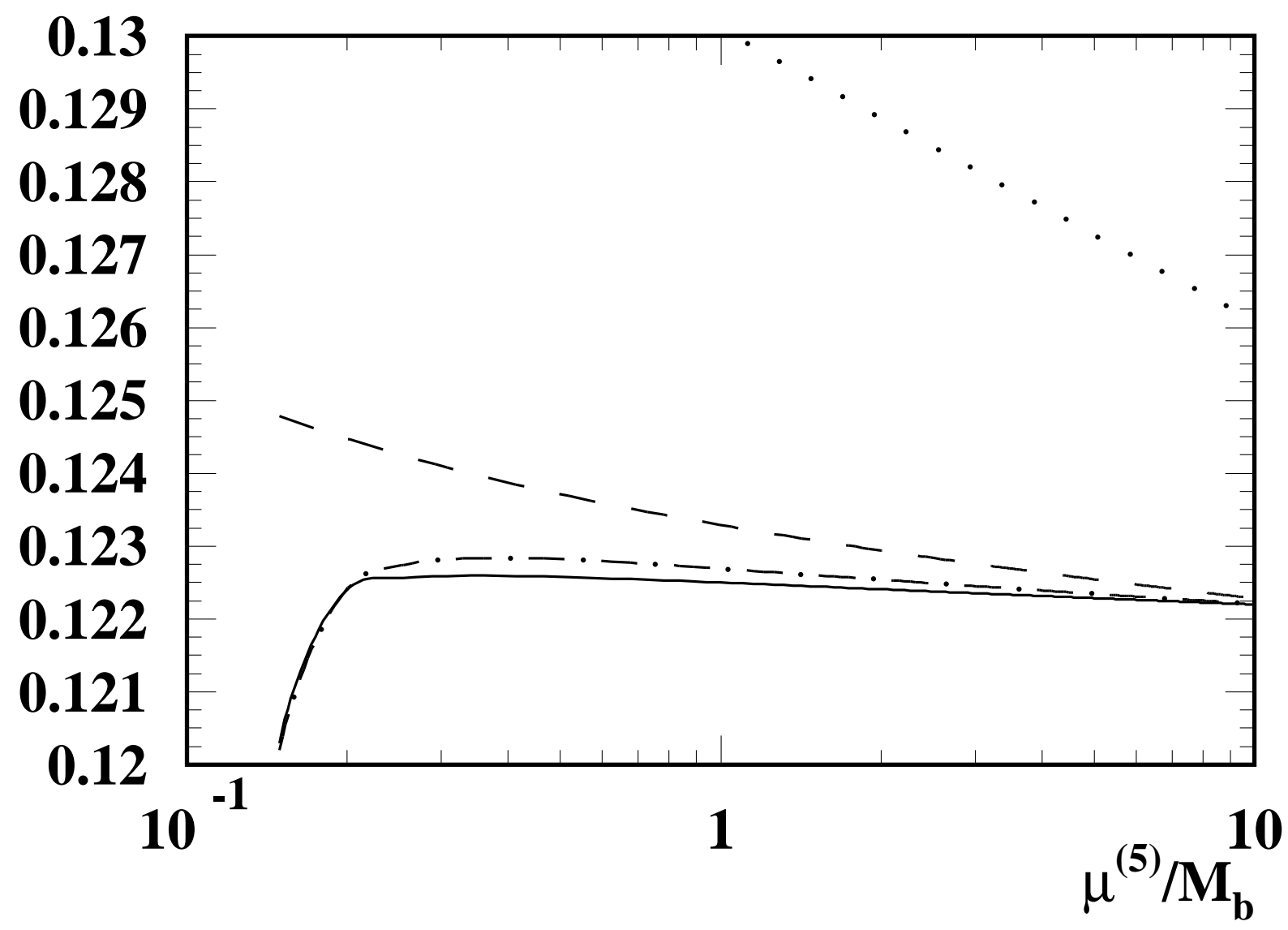

Figure 3.4: $\quad \mu^{(5)}$ dependence of $\alpha_{s}^{(5)}\left(M_{Z}\right)$ calculated from $\alpha_{s}^{(4)}\left(M_{\tau}\right)=0.36$ and $M_{b}=$ $4.7 \mathrm{GeV}$ using Eq. (2.35) at one (dotted), two (dashed), three (dot-dashed), and four (solid) loops in connection with Eqs. (3.4) and (3.30) at the respective orders.

\section{Scalar dimension-four operators in QCD}

In the last section we constructed an effective QCD Lagrangian which results from integrating out a heavy quark. From the knowledge of its structure we determined the coefficient functions (i.e. the decoupling constants) by computing bare Green functions in the full and effective theory.

A different point of view is adopted for the construction of the so-called non-relativistic QCD (NRQCD) 655. It has been developed in the context of heavy quarkonium physics in order to separate the relativistic scales associated with the mass of the heavy quark, $M$, from the non-relativistic ones which are of the order $M v$, where $v$ is the velocity of the quark. As a result one obtains an effective Lagrangian which is ordered in inverse 


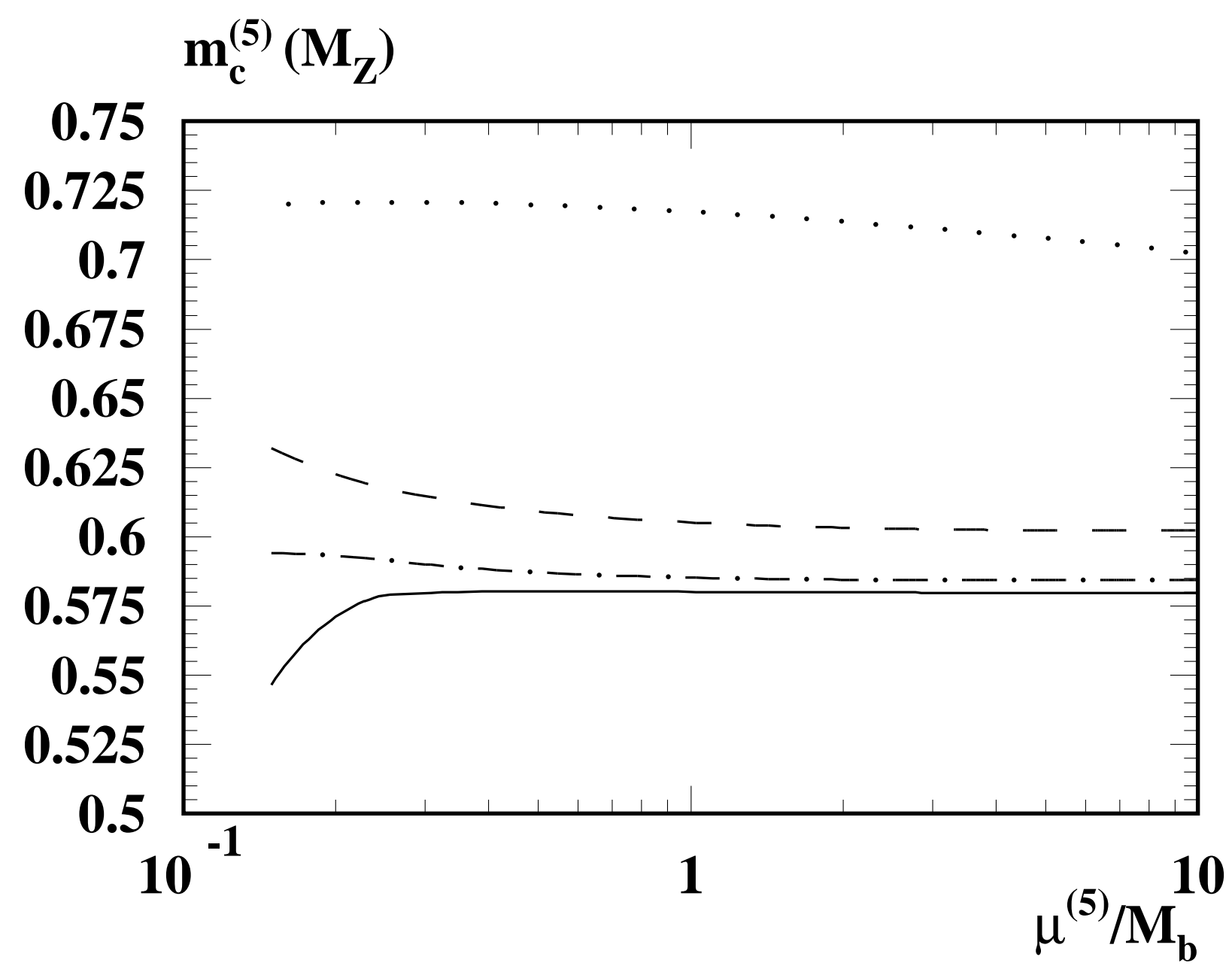

Figure 3.5: $\mu^{(5)}$ dependence of $m_{c}^{(5)}\left(M_{Z}\right)$ calculated from $\mu_{c}=m_{c}^{(4)}\left(\mu_{c}\right)=1.2 \mathrm{GeV}$, $M_{b}=4.7 \mathrm{GeV}$, and $\alpha_{s}^{(5)}\left(M_{Z}\right)=0.118$ using Eq. (2.12) at one (dotted), two (dashed), three (dot-dashed), and four (solid) loops in connection with Eqs. (3.4) and (3.28) at the respective orders.

powers of $M$. More recently a further step has been undertaken and potential NRQCD (pNRQCD) has been introduced in order to account for the separation of the scales $M v$ and $M v^{2}$. For recent overviews we refer to [66, 67].

In this section a different approach is considered. It is based on Wilson's operator product expansion (OPE) [68 which is a powerful method for the construction of effective theories. The basic idea is to introduce local operators $\mathcal{O}_{i}$ of appropriate dimension. They are formed by the light degrees of freedom and parameterize the long-distance behaviour. The operators are accompanied by coefficient functions which contain the remnant of the large parameters of the theory.

As a simple example of the OPE one can consider the decay amplitude of the muon in the SM. Due to the fact that the momentum transfer is much smaller than the mass 
of the $W$ boson, $M_{W}$, the former can be neglected with respect to the latter. This leads to the famous four-fermion interaction which is generated from dimension-six operators. Thus, for dimensional reasons the coefficient functions contain a factor $1 / M_{W}^{2}$.

In QCD the scalar operators of dimension four have been studied in great detail [69, 70, 71]. A comprehensive survey concerning their renormalization properties and mixings is performed in 71]. In particular, all renormalization constants of the operators have been expressed in terms of renormalization constants which already appear in the QCD Lagrangian (cf. Eq. (3.3)).

In this Section we want to discuss two applications of the scalar dimension-four operators. In the first one we consider the decay of an intermediate-mass Higgs boson with $M_{W} \lesssim M_{H} \lesssim 2 M_{W}$ into quarks or gluons. Here the top quark is considered to be heavy and thus it will only contribute to the coefficient functions. On the other hand, the scale in the operators is given by the mass of the Higgs boson. A somehow complementary situation is considered in the second example: the quartic quark mass corrections to the vector current correlators. Here the mass of the quark sets the scale in the operators. It is considered to be small as compared to the external momentum which manifests itself in the coefficient functions.

For definiteness we want to list the operators of dimension four in this introductory part and briefly discuss their renormalization. In [71] the operators are classified into gaugeinvariant and non-gauge-invariant ones. Furthermore a distinction is made whether the operators vanish or not by virtue of the equation of motion. The gauge-invariant operators readm

$$
\begin{aligned}
\mathcal{O}_{1}^{0} & =\left(G_{\mu \nu}^{0, \prime, a}\right)^{2} \\
\mathcal{O}_{2}^{0} & =m_{q}^{0, \prime} \bar{\psi}_{q}^{0, \prime} \psi_{q}^{0, \prime} \\
\mathcal{O}_{3}^{0} & =\bar{\psi}_{q}^{0, \prime}\left(i \not D^{0, \prime}-m_{q}^{0, \prime}\right) \psi_{q}^{0, \prime} \\
\mathcal{O}_{6}^{0} & =\left(m_{q}^{0, \prime}\right)^{4}
\end{aligned}
$$

where $G_{\mu \nu}^{0, a}$ and $D_{\mu}$ are defined after Eq. (3.1). Note that $\mathcal{O}_{3}^{0}$ vanishes after the application of the equation of motion.

In order to obtain a closed system two more operators have to be considered

$$
\begin{aligned}
\mathcal{O}_{4}^{0} & =G_{\nu}^{0,, a}\left(\nabla_{\mu}^{a b} G^{0, \prime, b \mu \nu}+g_{s}^{0, \prime} \sum_{i=1}^{n_{l}} \bar{\psi}_{q_{i}}^{0, \prime} \frac{\lambda^{a}}{2} \gamma^{\nu} \psi_{q_{i}}^{0, \prime}\right)-\partial_{\mu} \bar{c}^{0,,, a} \partial^{\mu} c^{0,,, a} \\
\mathcal{O}_{5}^{0} & =\left(\nabla_{\mu}^{a b} \partial^{\mu} \bar{c}^{0, \prime, b}\right) c^{0, \prime, a}
\end{aligned}
$$

where $\nabla_{\mu}^{a b}=\delta^{a b} \partial_{\mu}-g_{s} f^{a b c} G_{\mu}^{c}$ is the covariant derivative acting on the gluon and ghost fields. The operators in Eq. (4.2) are not gauge-invariant and thus do not contribute to physical observables.

\footnotetext{
${ }^{11}$ For consistency, the operators should also have a prime as a superscript as they are built by quantities of the effective theory. However, we refrain from introducing this additional index.
} 
From the practical point of view the operators of Eqs. (4.1) and (4.2) define new Feynman rules which can be read off from Eqs. (4.1) and (4.2). E.g., in Section 4.1.4 we have to compute the correlator $\left\langle\mathcal{O}_{1} \mathcal{O}_{1}\right\rangle$ up to three-loop order. This makes it necessary to extract the Feynman rules for the coupling of $\mathcal{O}_{1}$ to one-, two-, three- and four gluons from Eq. (4.1). We refrain from listing them explicitly but consider as illustrative examples the coupling of $\mathcal{O}_{1}^{0}$ and $\mathcal{O}_{4}^{0}$ to two gluons. This will be useful further below in Section 4.1 in order to demonstrate the evaluation of the corresponding coefficient functions.

The coupling to two gluons is obtained from those terms of Eqs. (4.1) and (4.2) containing two gluon fields. In the case of $\mathcal{O}_{1}^{0}$ and $\mathcal{O}_{4}^{0}$ they read

$$
\begin{aligned}
& \left.\mathcal{O}_{1}^{0}\right|_{\text {two gluons }}=2\left(\partial_{\mu} G_{\nu}^{0,, a} \partial^{\mu} G^{\nu, 0, l, b}-\partial_{\mu} G_{\nu}^{0,,, a} \partial^{\nu} G^{\mu, 0, r, b}\right), \\
& \left.\mathcal{O}_{4}^{0}\right|_{\text {two gluons }}=G_{\nu}^{0,,, a} \square G^{\nu, 0, \ell, b}-G_{\nu}^{0, l, a} \partial^{\nu} \partial_{\mu} G^{\mu, 0, \ell, b} .
\end{aligned}
$$

If we adopt the momenta flow as shown in Fig. 4.1 this results in the following Feynman rules for the vertices

$$
\begin{aligned}
V_{g, \mathcal{O}_{1}}^{\mu \nu, a}\left(p_{1}, p_{2}\right) & =i \delta^{a b} 4\left[-g^{\mu \nu} p_{1} \cdot p_{2}+p_{1}^{\nu} p_{2}^{\mu}\right], \\
V_{g g \mathcal{O}_{4}}^{\mu \nu, a b}\left(p_{1}, p_{2}\right) & =i \delta^{a b}\left[-g^{\mu \nu}\left(p_{1} \cdot p_{1}+p_{2} \cdot p_{2}\right)+p_{2}^{\mu} p_{2}^{\nu}+p_{1}^{\mu} p_{1}^{\nu}\right]
\end{aligned}
$$

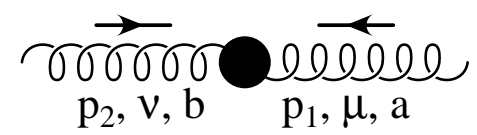

Figure 4.1: Coupling to two gluons. The solid circle either represents $\mathcal{O}_{1}^{0}$ or $\mathcal{O}_{4}^{0}$ and $a$ and $b$ are the colour indices of the gluons. The momenta $p_{1}$ and $p_{2}$ are incoming.

In the applications we discuss below the operators $\mathcal{O}_{3}, \mathcal{O}_{4}$ and $\mathcal{O}_{5}$ and the corresponding coefficient functions only appear in bare form. Thus we will not specify their renormalization, which can be found in [71], and concentrate in the following on $\mathcal{O}_{1}, \mathcal{O}_{2}$ and $\mathcal{O}_{6}$. The relation between the bare and the renormalized operators reads

$$
\mathcal{O}_{n}=\sum_{m} Z_{n m} \mathcal{O}_{m}^{0},
$$

where the indices $n$ and $m$ adopt the values 1,2 and 6 . Due to the equality

$$
\sum_{n} C_{n}^{0} \mathcal{O}_{n}^{0}=\sum_{n} C_{n} \mathcal{O}_{n},
$$

we obtain the renormalization prescription for the coefficient functions as

$$
C_{n}=\sum_{m}\left(Z^{-1}\right)_{m n} C_{m}^{0} .
$$


The anomalous dimension matrix pertaining to $Z_{n m}$ is defined through

$$
\mu^{2} \frac{\mathrm{d}}{\mathrm{d} \mu^{2}} \mathcal{O}_{n}=\sum_{m} \gamma_{m n} \mathcal{O}_{m} .
$$

It is connected to the renormalization matrix through

$$
\gamma_{n m}=\sum_{k}\left(\mu^{2} \frac{\mathrm{d}}{\mathrm{d} \mu^{2}} Z_{m k}\right)\left(Z^{-1}\right)_{k n},
$$

and reads in explicit form [69, 70, 71]

$$
\gamma=\left(\begin{array}{ccc}
-\alpha_{s} \pi \frac{\partial}{\partial \alpha_{s}} \frac{\beta}{\alpha_{s}} & 4 \alpha_{s} \frac{\partial}{\partial \alpha_{s}} \gamma_{m} & 4 \alpha_{s} \frac{\partial}{\partial \alpha_{s}} \gamma_{0} \\
0 & 0 & -4 \gamma_{0} \\
0 & 0 & 4 \gamma_{m}
\end{array}\right)
$$

Thus $\gamma_{n m}$ can be expressed in terms of the functions $\beta\left(\alpha_{s}\right)$ (see Eq. (2.35)), $\gamma_{m}\left(\alpha_{s}\right)$ (see Eq. (2.12)) and the anomalous dimension of the vacuum energy $\gamma_{0}$ which is given by [72, 73, [37]

$$
\begin{aligned}
\gamma_{0}= & -\frac{3}{16 \pi^{2}}\left\{1+\frac{4}{3} \frac{\alpha_{s}}{\pi}+\left(\frac{\alpha_{s}}{\pi}\right)^{2}\left[\frac{313}{72}-\frac{2}{3} \zeta_{3}-\frac{5}{12} n_{f}\right]\right. \\
& +\left(\frac{\alpha_{s}}{\pi}\right)^{3}\left[\frac{14251}{1296}-\frac{77}{2} \zeta_{3}+\frac{19}{6} \zeta_{4}+\frac{1975}{54} \zeta_{5}\right. \\
& \left.+n_{f}\left(-\frac{4109}{1944}-\frac{35}{54} \zeta_{3}-\frac{16}{9} \zeta_{4}\right)+n_{f}^{2}\left(-\frac{341}{1458}+\frac{2}{9} \zeta_{3}\right)\right],
\end{aligned}
$$

where $n_{f}$ is the number of active quark flavours.

In the formalism presented above it is assumed that there are only currents coupling to quarks of the same flavour which is sufficient for the purpose discussed below. The more general case involving also non-diagonal terms can be found in Ref. [74].

\subsection{Hadronic Higgs decay}

The coupling of a scalar CP-even Higgs boson to quarks has a very simple structure as no $\gamma$ matrix is involved. It essentially consists of a factor containing the mass of the quarks and the coupling constant. This fact makes it very simple to construct an effective Lagrangian and to derive powerful low-energy theorems as we will see in this Subsection.

We want to consider QCD corrections to the hadronic decay of a Higgs boson in the so-called intermediate mass range, that is we compute the partial decay widths into quarks and gluons assuming that the top quark is much heavier than all other scales involved in the process. Although we have in mind the top quark as the heavy particle we will in the following consider a generic heavy quark with mass $m_{h}$.

To lowest order in the inverse heavy quark mass the effective Lagrangian is constructed by the operators of dimension four which are discussed above. As we consider light quark 
mass effects at most in leading order (which corresponds to an overall quadratic factor in the case $H \rightarrow q \bar{q}$ and to quark mass zero in the gluonic case) there is no contribution from the operator $\mathcal{O}_{6}$ and thus the anomalous dimension matrix becomes two dimensional.

We consider a theory where we have in addition to the QCD Lagrangian of Eq. (3.1) a scalar particle, $H$, which couples to fermions via

$$
\mathcal{L}_{Y}=-\frac{H^{0}}{v^{0}} \sum_{q} m_{q}^{0} \bar{\psi}_{q}^{0} \psi_{q}^{0},
$$

where the sum runs over all quark flavours. In the limit $m_{h} \rightarrow \infty$ Eq. (4.12) can be written as a sum over the operators given in Eqs. (4.1) and (4.2) accompanied by coefficient functions containing the residual dependence on the top quark:

$$
\mathcal{L}_{Y, \mathrm{eff}}=-\frac{H^{0}}{v^{0}} \sum_{i=1}^{5} C_{i}^{0} \mathcal{O}_{i}^{0} .
$$

The relation of the bare coefficient functions and the operators to their renormalized counterparts can be extracted form the anomalous dimension matrix given in Eq. (4.10). One obtains for the renormalized operators

$$
\begin{aligned}
\mathcal{O}_{1} & =\left[1+2\left(\frac{\alpha_{s}^{\prime} \partial}{\partial \alpha_{s}^{\prime}} \ln Z_{g}^{\prime}\right)\right] \mathcal{O}_{1}^{0}-4\left(\frac{\alpha_{s}^{\prime} \partial}{\partial \alpha_{s}^{\prime}} \ln Z_{m}^{\prime}\right) \mathcal{O}_{2}^{0} \\
\mathcal{O}_{2} & =\mathcal{O}_{2}^{0}
\end{aligned}
$$

and accordingly for the coefficient functions

$$
\begin{aligned}
C_{1} & =\frac{1}{1+2\left(\alpha_{s}^{\prime} \partial / \partial \alpha_{s}^{\prime}\right) \ln Z_{g}^{\prime}} C_{1}^{0}, \\
C_{2} & =\frac{4\left(\alpha_{s}^{\prime} \partial / \partial \alpha_{s}^{\prime}\right) \ln Z_{m}^{\prime}}{1+2\left(\alpha_{s}^{\prime} \partial / \partial \alpha_{s}^{\prime}\right) \ln Z_{g}^{\prime}} C_{1}^{0}+C_{2}^{0} .
\end{aligned}
$$

Consequently, the physical part of $\mathcal{L}_{Y, \text { eff }}$ takes the form

$$
\mathcal{L}_{Y, \text { eff }}^{\text {phys }}=-\frac{H^{0}}{v^{0}}\left(C_{1} \mathcal{O}_{1}+C_{2} \mathcal{O}_{2}\right)
$$

$\alpha_{s}^{\prime}, Z_{g}^{\prime}$ and $Z_{m}^{\prime}$ are defined in the effective theory which is indicated by the prime. $C_{i}$ and $\mathcal{O}_{i}(i=1,2)$ are individually finite, but, with the exception of $\left[\mathcal{O}_{2}^{\prime}\right]$, they are not separately renormalization-group $(\mathrm{RG})$ invariant. In Ref. [75], a RG-improved version of Eq. (4.16) has been constructed by exploiting the RG-invariance of the trace of the energy-momentum tensor. The ratio $H^{0} / v^{0}$ receives a finite renormalization factor, which is of $\mathcal{O}\left(G_{F} M_{t}^{2}\right)$. Its two- and three-loop QCD corrections have been found in Refs. [76] and [77], respectively.

A closer look to the operators in Eqs. (4.1) and (4.2) and the effective Lagrangian in Eq. (4.13) suggests that there should be a connection between the coefficient functions 
on one side and the decoupling constants as introduced in Eq. (3.4) on the other side. Actually it turns out that $C_{1}$ and $C_{2}$ are obtained from derivatives of $\ln \zeta_{g}$ and $\ln \zeta_{m}$, respectively. This nice feature allows for the computation of $C_{1}$ and $C_{2}$ to order $\alpha_{s}^{n}$ from the knowledge of $\zeta_{g}$ and $\zeta_{m}$ to order $\alpha_{s}^{n-1}$ [49] as we will see below.

The coefficient functions contain the remnants of the heavy quark. It is thus quite plausible that their computation can be reduced to Feynman diagrams where the only scale is given by $m_{h}$. Actually, the philosophy for the derivation of formulae for $C_{1}^{0}, \ldots, C_{5}^{0}$ is very similar to the case of the decoupling relations. One again considers Green functions in the full and effective theories and exploits the fact that the coefficient functions do not depend on the momentum configuration. We will see that for a certain Green function in general several coefficient functions are involved. Thus we will obtain five equations which can be solved for $C_{1}^{0}, \ldots, C_{5}^{0}$.

From Eqs. (4.1) and (4.2) one learns that the Green function involving one gluon, two ghosts and a zero-momentum insertion of the operator $\mathcal{O}_{h}=m_{h}^{0} \bar{h}^{0} h^{0}$, which mediates the coupling to the Higgs boson, only gets contributions from $\mathcal{O}_{5}^{0}$. We define the bare 1PI Green function in analogy to Eq. (3.7)

$$
\begin{aligned}
& p^{\mu} g_{s}^{0}\left\{-i f^{a b c}\left[\Gamma_{G \bar{c} c \mathcal{O}_{h}}^{0}(p, k, 0)\right]+\ldots\right\} \\
& \quad=i^{2} \int \mathrm{d} x \mathrm{~d} y e^{i(p \cdot x+k \cdot y)}\left\langle T c^{0, a}(x) \bar{c}^{0, b}(0) G^{0, c \mu}(y) \mathcal{O}_{h}(0)\right\rangle^{1 \mathrm{PI}},
\end{aligned}
$$

where the ellipses again represent other colour structures and $p$ and $k$ are the outgoing momenta of the $c$ and $G$, respectively. The third argument of $\Gamma_{G \bar{c} c \mathcal{O}_{h}}^{0}$ indicates the zero momentum of the operator $\mathcal{O}_{h}$.

In a next step we express $\mathcal{O}_{h}$ in terms of the operators given in Eqs. (4.1) and (4.2). Only $\mathcal{O}_{5}^{0}$ has to be taken into account as only this operator contains the coupling of a gluon to two ghost fields. In the transitition to the effective theory also Eq. (3.4) has to be considered which leads to a factor $\zeta_{g}^{0} /\left(\tilde{\zeta}_{3}^{0} \sqrt{\zeta_{3}^{0}}\right)$. Note that in Eq. (4.17) we are dealing with amputated Green functions. Thus from the external propagators a factor $\left(\tilde{\zeta}_{3}^{0}\right)^{2} \zeta_{3}^{0}$ arise. Finally we arrive at

$$
\begin{aligned}
p^{\mu} g_{s}^{0}\left(-i f^{a b c}\right) \Gamma_{G \bar{c} c \mathcal{O}_{h}}(p, k, 0)+\ldots & =p^{\mu} g_{s}^{0}\left(-i f^{a b c}\right) \zeta_{g}^{0} \tilde{\zeta}_{3}^{0} \sqrt{\zeta_{3}^{0}} C_{5}^{0}+\ldots \\
& =p^{\mu} g_{s}^{0}\left(-i f^{a b c}\right) \tilde{\zeta}_{1}^{0} C_{5}^{0}+\ldots
\end{aligned}
$$

where the ellipses represent other colour structures and terms suppressed by the inverse heavy quark mass. On the right-hand side of Eq. (4.18) we also avoided to write down explicitly the contributions beyond tree-level as they vanish for $p, k \rightarrow 0$ within dimensional regularization. In this limit only the diagrams involving the heavy quark survive on the left-hand side and we are left with the formula

$$
\tilde{\zeta}_{1}^{0} C_{5}^{0}=\Gamma_{G \bar{c} c \mathcal{O}_{h}}^{0, h}(0,0,0) .
$$

In order to reduce the number of contributing diagrams and also to simplify their complexity we exploit that the coupling of the Higgs boson is proportional to $m_{h}$. Thus 
it can simply be generated by differentiation with respect to $m_{h}$. With the definition

$$
\partial_{h}^{0}=\frac{\left(m_{h}^{0}\right)^{2} \partial}{\partial\left(m_{h}^{0}\right)^{2}},
$$

we finally obtain

$$
\tilde{\zeta}_{1}^{0} C_{5}^{0}=2 \partial_{h}^{0} \Gamma_{G \bar{c} c}^{0, h}(0,0)
$$

As another example, let us consider the derivation of a formula involving $C_{1}^{0}$ and $C_{4}^{0}$. The starting point is the $1 \mathrm{PI}$ Green function of two gluons which contains a zeromomentum insertion of the composite operator $\mathcal{O}_{h}$. In momentum space, it reads in bare form

$$
\begin{aligned}
\delta^{a b} \Gamma_{G G \mathcal{O}_{h}}^{0, \mu \nu}(p,-p) & =i^{2} \int \mathrm{d} x \mathrm{~d} y e^{i p \cdot(x-y)}\left\langle T G^{0, a, \mu}(x) G^{0, b, \nu}(y) \mathcal{O}_{h}(0)\right\rangle^{1 \mathrm{PI}} \\
& =\delta^{a b}\left[-g^{\mu \nu} p^{2} \Gamma_{G G \mathcal{O}_{h}}^{0}\left(p^{2}\right)+\text { terms proportional to } p^{\mu} p^{\nu}\right]
\end{aligned}
$$

where $p$ denotes the four-momentum flowing along the gluon line. In the limit $m_{h} \rightarrow \infty$, $\mathcal{O}_{h}$ may be written as a linear combination of the effective operators given in Eqs. (4.1) and (4.2), so that

$$
\begin{aligned}
\Gamma_{G G \mathcal{O}_{h}}^{0, \mu \nu}(p,-p) & =-g^{\mu \nu} p^{2} \Gamma_{G G \mathcal{O}_{h}}^{0}(p,-p)+\ldots \\
& =\frac{i^{2}}{8} \int \mathrm{d} x \mathrm{~d} y e^{i p \cdot(x-y)}\left\langle T G^{0, a, \mu}(x) G^{0, a, \nu}(y)\left(C_{1}^{0} \mathcal{O}_{1}^{0}+C_{4}^{0} \mathcal{O}_{4}^{0}\right)\right\rangle^{1 \mathrm{PI}}+\ldots \\
& =\frac{i^{2}}{8} \zeta_{3}^{0} \int \mathrm{d} x \mathrm{~d} y e^{i p \cdot(x-y)}\left\langle T G^{0 \prime, a, \mu}(x) G^{0 \prime, a, \nu}(y)\left(C_{1}^{0} \mathcal{O}_{1}^{0}+C_{4}^{0} \mathcal{O}_{4}^{0}\right)\right\rangle^{1 \mathrm{PI}}+\ldots \\
& =-g^{\mu \nu} p^{2} \zeta_{3}^{0}\left(-4 C_{1}^{0}+2 C_{4}^{0}\right)(1+\text { higher orders })+\ldots
\end{aligned}
$$

where the ellipses indicate terms of $\mathcal{O}\left(1 / m_{h}\right)$ and terms proportional to $p^{\mu} p^{\nu}$. The factor $1 / 8$ results for the summation over the colour indices. In the second step, we have used Eq. (3.4) together with the fact that $\Gamma_{G G \mathcal{O}_{h}}^{0, \mu \nu}(p,-p)$ represents an amputated Green function. If we consider the coefficients of the transversal part in the limit $p \rightarrow 0$, we observe that the contributions due to the higher-order QCD corrections on the right-hand side of Eq. (4.23) vanish, as massless tadpoles are set to zero in dimensional regularization. In principle also the other operators contribute via loop diagrams. However, also these contributions lead to massless tadpoles and are thus zero. The relative weight between $C_{1}^{0}$ and $C_{4}^{0}$ and the prefactor in the last line of Eq. (4.23) follow immediately from the Feynman rules given in Eq. (4.4). On the left-hand side, only those diagrams survive which contain at least one heavy-quark line. Consequently, the hard part of the amputated Green function is given by

$$
\Gamma_{G G \mathcal{O}_{h}}^{0, h}(0,0)=\zeta_{3}^{0}\left(-4 C_{1}^{0}+2 C_{4}^{0}\right)
$$

\footnotetext{
${ }^{12}$ We want to mention that in Ref. [49] there are misprints in the corresponding formulae: erroneously they contain a factor " $1 / 2$ " instead of "2". However, the initial equations and the final results are correct in [49].
} 
Using Eq. (4.20) we finally arrive at

$$
\zeta_{3}^{0}\left(-4 C_{1}^{0}+2 C_{4}^{0}\right)=-2 \partial_{h}^{0} \Pi_{G}^{0, h}(0) .
$$

In a similar way, we obtain three more relationships, namely

$$
\begin{aligned}
\zeta_{2}^{0} C_{3}^{0} & =-2 \partial_{h}^{0} \Sigma_{V}^{0, h}(0), \\
\zeta_{m}^{0} \zeta_{2}^{0}\left(C_{2}^{0}-C_{3}^{0}\right) & =1-\Sigma_{S}^{0, h}(0)-2 \partial_{h}^{0} \Sigma_{S}^{0, h}(0), \\
\tilde{\zeta}_{3}^{0}\left(C_{4}^{0}+C_{5}^{0}\right) & =2 \partial_{h}^{0} \Pi_{c}^{0, h}(0) .
\end{aligned}
$$

The Eqs. (4.21), (4.25) and (4.26) may now be solved for the coefficient functions $C_{i}^{0}$ $(i=1, \ldots, 5)$. They are expressed in terms of vacuum integrals with only one mass scale, namely the heavy quark mass. This is also the case for the decoupling constants occuring in Eqs. (4.21), (4.25) and (4.26) as discussed in Section 3.

As a simple example let us consider the computation of $C_{1}$ at lowest order. Here only one diagram contributes to $\Pi_{G}^{0, h}(0)$, namely the one where the gluon splits into two virtual heavy quarks. It can be evaluated with the help of Eq. (A.6). Expanded up to finite order in $\varepsilon$ it reads

$$
\Pi_{G}^{0, h}(0)=\frac{\alpha_{s}^{0}}{\pi} T\left(\frac{1}{3 \varepsilon}+\frac{1}{3} \ln \frac{\mu^{2}}{\left(m_{h}^{0}\right)^{2}}\right) .
$$

Differentiating with respect to $m_{h}^{0}$ leads to

$$
C_{1}=-\frac{\alpha_{s}}{\pi} \frac{T}{6} .
$$

Note, that at this order no renormalization is needed and thus the bare and renormalized quantities coincide. Furthermore, there is no contribution from $\zeta_{3}^{0}$ in Eq. (4.25) and $C_{4}^{0}$ contributes for the first time at order $\alpha_{s}^{2}$ as can be seen from Eqs. (4.26).

In the next three Subsections we will describe different methods to compute the coefficient functions. Finally in Section 4.1 .4 we will review the state-of-the-art corrections to the hadronic decay width of the Higgs boson.

\subsubsection{Direct calculation of the coefficient functions}

There is the possibility to avoid the occurrence of the non-physical operators and their coefficient functions in the computation of $C_{1}^{0}$ and $C_{2}^{0}$. For demonstration let us consider the case of $C_{1}^{0}$.

From the definition of the operators in Eqs. (4.1) and (4.2) and the Feynman rules given in (4.4) one can read off that the 1PI Green function $\Gamma_{G G \mathcal{O}_{h}}^{0, \mu \nu}\left(p_{1}, p_{2}\right)$, where $p_{1}$ and $p_{2}$ denote the incoming momenta of the gluons, only receives contributions from $C_{1}^{0} \mathcal{O}_{1}^{0}$ if $p_{1} \neq-p_{2}$. In analogy to Eq. (4.22) we can write

$$
\begin{aligned}
\Gamma_{G G \mathcal{O}_{h}}^{0, \mu \nu}\left(p_{1}, p_{2}\right) & =\left(g^{\mu \nu} p_{1} \cdot p_{2}-p_{1}^{\nu} p_{2}^{\mu}\right) \Gamma_{G G \mathcal{O}_{h}}^{0}\left(p_{1}, p_{2}\right) \\
& =\left(g^{\mu \nu} p_{1} \cdot p_{2}-p_{1}^{\nu} p_{2}^{\mu}\right) \zeta_{3}^{0}\left(-4 C_{1}^{0}\right)(1+\ldots)
\end{aligned}
$$


where the ellipses denote terms of order $1 / m_{h}$, possible other Lorentz structures and higher order loop corrections. The latter vanish in the limit $p_{1}, p_{2} \rightarrow 0$ and we are left with

$$
\begin{aligned}
\zeta_{3}^{0} C_{1}^{0} & =-\frac{1}{4} \Gamma_{G G \mathcal{O}_{h}}^{0, h}(0,0) \\
& =-\left.\frac{1}{4}\left(\frac{g_{\mu \nu} p_{1} \cdot p_{2}-p_{1, \nu} p_{2, \mu}-p_{1, \mu} p_{2, \nu}}{(D-2)\left(p_{1} \cdot p_{2}\right)^{2}} \Gamma_{G G \mathcal{O}_{h}}^{0, h, \mu \nu}\left(p_{1}, p_{2}\right)\right)\right|_{p_{1}=p_{2}=0},
\end{aligned}
$$

where the on-shell conditions $p_{1}^{2}=p_{2}^{2}=0$ have been used. This equation relates the coefficient function $C_{1}^{0}$ directly to a physical amplitude and no Green functions involving ghost fields have to be considered. The price one has to pay is that the momenta $p_{1}$ and $p_{2}$ can only be set to zero after the projection in Eq. (4.30) has been applied. This complicates the expressions of the individual diagrams. Furthermore it is not possible to use derivatives with respect to $m_{h}^{0}$ in order to generate the coupling to the Higgs boson as initially the momentum of the Higgs boson is not zero. Thus altogether 657 vertex diagrams like the first one in Fig. 4.2 have to be considered.
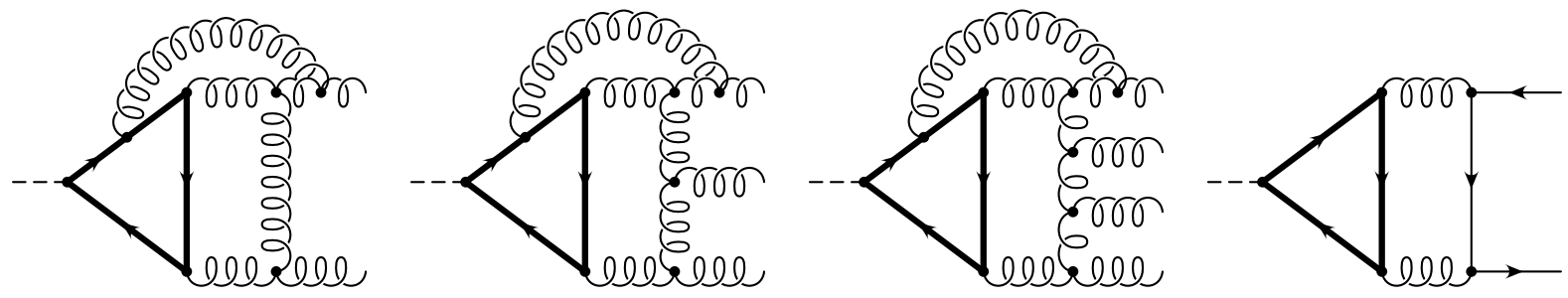

Figure 4.2: Typical Feynman diagrams contributing to the coefficients $C_{1}^{0}$ and $C_{2}^{0}$. Looped, bold-faced, and dashed lines represent gluons, heavy quarks, and Higgs bosons, respectively.

In analogy to the gluon-gluon-Higgs boson three-point function one could also choose the part of the operator $\mathcal{O}_{1}$ involving three or even four gluons. This would lead to 7362 four- and 95004 five-point functions at three-loop level, where one and no gluon momentum, respectively, has to be kept different from zero until the projection is finished. Sample diagrams are pictured in Fig. 4.2. In Ref. [78], where the decay of a pseudo-scalar Higgs boson has been considered, the 7362 three-loop four-point functions have been evaluated in order to check the calculation of the three-point function. For completeness we want to mention that the last diagram in Fig. 4.2 is the lowest-order graph contributing to $C_{2}$.

\subsubsection{Low-energy theorem}

It is tempting to re-express the Green functions on the right-hand side of Eqs. (4.21), (4.25) and (4.26) in terms of the decoupling constants which were considered in Section 3 . This leads to a straight relation between the coefficient functions and the decoupling constants. 
For the equations involving $C_{1}^{0}, C_{4}^{0}$ and $C_{5}^{0}$ one successively obtains

$$
\begin{aligned}
C_{5}^{0} & =2 \frac{\partial_{h}^{0} \tilde{\zeta}_{1}^{0}}{\tilde{\zeta}_{1}^{0}}, \\
C_{4}^{0} & =2\left(\frac{\partial_{h}^{0} \tilde{\zeta}_{3}^{0}}{\tilde{\zeta}_{3}^{0}}-\frac{\partial_{h}^{0} \tilde{\zeta}_{1}^{0}}{\tilde{\zeta}_{1}^{0}}\right), \\
C_{1}^{0} & =\frac{1}{2} \frac{\partial_{h}^{0} \zeta_{3}^{0}}{\zeta_{3}^{0}}+\frac{\partial_{h}^{0} \tilde{\zeta}_{3}^{0}}{\tilde{\zeta}_{3}^{0}}-\frac{\partial_{h}^{0} \tilde{\zeta}_{1}^{0}}{\tilde{\zeta}_{1}^{0}} \\
& =-\partial_{h}^{0} \ln \frac{\tilde{\zeta}_{1}^{0}}{\tilde{\zeta}_{3}^{0} \sqrt{\zeta_{3}^{0}}} \\
& =-\partial_{h}^{0} \ln \zeta_{g}^{0},
\end{aligned}
$$

Next, we express $\zeta_{g}^{0}$ through renormalized quantities. Using $\partial_{h}^{0}=\partial_{h}$, we find

$$
\begin{aligned}
-2 C_{1}^{0} & =\partial_{h} \ln \left(\zeta_{g}^{0}\right)^{2} \\
& =\partial_{h} \ln \frac{\alpha_{s}^{0 \prime}}{\alpha_{s}^{0}} \\
& =\partial_{h} \ln \left(Z_{g}^{\prime}\right)^{2}+\partial_{h} \ln \alpha_{s}^{\prime} \\
& =\left[1+\frac{\alpha_{s}^{\prime} \partial}{\partial \alpha_{s}^{\prime}} \ln \left(Z_{g}^{\prime}\right)^{2}\right] \partial_{h} \ln \alpha_{s}^{\prime} .
\end{aligned}
$$

Identifying the renormalization factor of Eq. (4.15), we obtain the amazingly simple relation

$$
\begin{aligned}
-2 C_{1} & =\partial_{h} \ln \alpha_{s}^{\prime} \\
& =\partial_{h} \ln \zeta_{g}^{2} .
\end{aligned}
$$

This relation opens the possibility to compute $C_{1}$ through $\mathcal{O}\left(\alpha_{s}^{4}\right)$, since one only needs to know the logarithmic contributions of $\zeta_{g}$ in this order, which may be reconstructed from its lower-order terms in combination with the four-loop $\beta$ [14] and $\gamma_{m}$ [15, 16] functions.

It is furthermore possible to directly relate $C_{1}$ to the $\beta$ and $\gamma_{m}$ functions of the full and effective theories. Exploiting the relation

$$
\begin{aligned}
\beta^{\prime}\left(\alpha_{s}^{\prime}\right) & =\frac{\mu^{2} d}{d \mu^{2}} \frac{\alpha_{s}^{\prime}}{\pi} \\
& =\left[\frac{\mu^{2} \partial}{\partial \mu^{2}}+\beta\left(\alpha_{s}\right) \frac{\partial}{\partial \alpha_{s}}+\gamma_{m}\left(\alpha_{s}\right) \frac{m_{h} \partial}{\partial m_{h}}\right] \frac{\alpha_{s}^{\prime}}{\pi}
\end{aligned}
$$

where $\alpha_{s}^{\prime}=\alpha_{s}^{\prime}\left(\mu, \alpha_{s}, m_{h}\right)$, we find

$$
C_{1}=\frac{\pi}{2 \alpha_{s}^{\prime}\left[1-2 \gamma_{m}\left(\alpha_{s}\right)\right]}\left[\beta^{\prime}\left(\alpha_{s}^{\prime}\right)-\beta\left(\alpha_{s}\right) \frac{\partial \alpha_{s}^{\prime}}{\partial \alpha_{s}}\right] .
$$


In the case of $C_{2}$, we may proceed along the same lines to obtain

$$
\begin{aligned}
C_{2} & =1+2 \partial_{h} \ln \zeta_{m} \\
& =1-\frac{2}{1-2 \gamma_{m}\left(\alpha_{s}\right)}\left[\gamma_{m}^{\prime}\left(\alpha_{s}^{\prime}\right)-\gamma_{m}\left(\alpha_{s}\right)-\beta\left(\alpha_{s}\right) \frac{1}{m_{q}^{\prime}} \frac{\partial m_{q}^{\prime}}{\partial \alpha_{s}}\right],
\end{aligned}
$$

where $m_{q}^{\prime}=m_{q}^{\prime}\left(\mu, \alpha_{s}, m_{h}\right)$. It should be stressed that Eqs. (4.35) and (4.36) are valid to all orders in $\alpha_{s}$.

Fully exploiting present knowledge of the $\beta$ [14] and $\gamma_{m}$ [15, 16] functions, we may evaluate $C_{1}$ and $C_{2}$ through $\mathcal{O}\left(\alpha_{s}^{4}\right)$ via Eqs. (4.35) and (4.36). In the pure $\overline{\mathrm{MS}}$ scheme, we find 79 , 49,

$$
\begin{aligned}
& C_{1}^{\mathrm{MS}}=-\frac{1}{12} \frac{\alpha_{s}^{\left(n_{f}\right)}(\mu)}{\pi}\left\{1+\frac{\alpha_{s}^{\left(n_{f}\right)}(\mu)}{\pi}\left(\frac{11}{4}-\frac{1}{6} \ln \frac{\mu^{2}}{m_{h}^{2}}\right)\right. \\
& +\left(\frac{\alpha_{s}^{\left(n_{f}\right)}(\mu)}{\pi}\right)^{2}\left[\frac{2821}{288}-\frac{3}{16} \ln \frac{\mu^{2}}{m_{h}^{2}}+\frac{1}{36} \ln ^{2} \frac{\mu^{2}}{m_{h}^{2}}+n_{l}\left(-\frac{67}{96}+\frac{1}{3} \ln \frac{\mu^{2}}{m_{h}^{2}}\right)\right] \\
& +\left(\frac{\alpha_{s}^{\left(n_{f}\right)}(\mu)}{\pi}\right)^{3}\left[-\frac{4004351}{62208}+\frac{1305893}{13824} \zeta_{3}-\frac{859}{288} \ln \frac{\mu^{2}}{m_{h}^{2}}+\frac{431}{144} \ln ^{2} \frac{\mu^{2}}{m_{h}^{2}}\right. \\
& -\frac{1}{216} \ln ^{3} \frac{\mu^{2}}{m_{h}^{2}}+n_{l}\left(\frac{115607}{62208}-\frac{110779}{13824} \zeta_{3}+\frac{641}{432} \ln \frac{\mu^{2}}{m_{h}^{2}}+\frac{151}{288} \ln ^{2} \frac{\mu^{2}}{m_{h}^{2}}\right) \\
& \left.\left.+n_{l}^{2}\left(-\frac{6865}{31104}+\frac{77}{1728} \ln \frac{\mu^{2}}{m_{h}^{2}}-\frac{1}{18} \ln ^{2} \frac{\mu^{2}}{m_{h}^{2}}\right)\right]\right\} \\
& \approx-\frac{1}{12} \frac{\alpha_{s}^{\left(n_{f}\right)}\left(\mu_{h}\right)}{\pi}\left[1+2.7500 \frac{\alpha_{s}^{\left(n_{f}\right)}\left(\mu_{h}\right)}{\pi}+\left(9.7951-0.6979 n_{l}\right)\left(\frac{\alpha_{s}^{\left(n_{f}\right)}\left(\mu_{h}\right)}{\pi}\right)^{2}\right. \\
& \left.+\left(49.1827-7.7743 n_{l}-0.2207 n_{l}^{2}\right)\left(\frac{\alpha_{s}^{\left(n_{f}\right)}\left(\mu_{h}\right)}{\pi}\right)^{3}\right], \\
& C_{2}^{\mathrm{MS}}=1+\left(\frac{\alpha_{s}^{\left(n_{f}\right)}(\mu)}{\pi}\right)^{2}\left(\frac{5}{18}-\frac{1}{3} \ln \frac{\mu^{2}}{m_{h}^{2}}\right) \\
& +\left(\frac{\alpha_{s}^{\left(n_{f}\right)}(\mu)}{\pi}\right)^{3}\left[\frac{311}{1296}+\frac{5}{3} \zeta_{3}-\frac{175}{108} \ln \frac{\mu^{2}}{m_{h}^{2}}-\frac{29}{36} \ln ^{2} \frac{\mu^{2}}{m_{h}^{2}}\right. \\
& \left.+n_{l}\left(\frac{53}{216}+\frac{1}{18} \ln ^{2} \frac{\mu^{2}}{m_{h}^{2}}\right)\right] \\
& +\left(\frac{\alpha_{s}^{\left(n_{f}\right)}(\mu)}{\pi}\right)^{4}\left[\frac{2800175}{186624}+\frac{373261}{13824} \zeta_{3}-\frac{155}{6} \zeta_{4}-\frac{575}{36} \zeta_{5}+\frac{31}{72} B_{4}\right. \\
& +\left(-\frac{50885}{2592}+\frac{155}{12} \zeta_{3}\right) \ln \frac{\mu^{2}}{m_{h}^{2}}-\frac{1219}{216} \ln ^{2} \frac{\mu^{2}}{m_{h}^{2}}-\frac{301}{144} \ln ^{3} \frac{\mu^{2}}{m_{h}^{2}}
\end{aligned}
$$




$$
\begin{aligned}
& +n_{l}\left(-\frac{16669}{15552}-\frac{221}{288} \zeta_{3}+\frac{25}{12} \zeta_{4}-\frac{1}{36} B_{4}+\frac{7825}{2592} \ln \frac{\mu^{2}}{m_{h}^{2}}+\frac{23}{48} \ln ^{2} \frac{\mu^{2}}{m_{h}^{2}}\right. \\
& \left.\left.+\frac{5}{18} \ln ^{3} \frac{\mu^{2}}{m_{h}^{2}}\right)+n_{l}^{2}\left(\frac{3401}{23328}-\frac{7}{54} \zeta_{3}-\frac{31}{324} \ln \frac{\mu^{2}}{m_{h}^{2}}-\frac{1}{108} \ln ^{3} \frac{\mu^{2}}{m_{h}^{2}}\right)\right] \\
& \approx 1+0.2778\left(\frac{\alpha_{s}^{\left(n_{f}\right)}\left(\mu_{h}\right)}{\pi}\right)^{2}+\left(2.2434+0.2454 n_{l}\right)\left(\frac{\alpha_{s}^{\left(n_{f}\right)}\left(\mu_{h}\right)}{\pi}\right)^{3} \\
& +\left(2.1800+0.3096 n_{l}-0.0100 n_{l}^{2}\right)\left(\frac{\alpha_{s}^{\left(n_{f}\right)}\left(\mu_{h}\right)}{\pi}\right)^{4},
\end{aligned}
$$

where, for simplicity, we have chosen $\mu=\mu_{h} \equiv m_{h}\left(\mu_{h}\right)$ in the approximate expressions. The corresponding results expressed in terms of the pole mass $M_{h}$ read

$$
\begin{aligned}
& C_{1}^{\mathrm{OS}}=-\frac{1}{12} \frac{\alpha_{s}^{\left(n_{f}\right)}(\mu)}{\pi}\left\{1+\frac{\alpha_{s}^{\left(n_{f}\right)}(\mu)}{\pi}\left(\frac{11}{4}-\frac{1}{6} \ln \frac{\mu^{2}}{M_{h}^{2}}\right)\right. \\
& +\left(\frac{\alpha_{s}^{\left(n_{f}\right)}(\mu)}{\pi}\right)^{2}\left[\frac{2693}{288}-\frac{25}{48} \ln \frac{\mu^{2}}{M_{h}^{2}}+\frac{1}{36} \ln ^{2} \frac{\mu^{2}}{M_{h}^{2}}+n_{l}\left(-\frac{67}{96}+\frac{1}{3} \ln \frac{\mu^{2}}{M_{h}^{2}}\right)\right] \\
& +\left(\frac{\alpha_{s}^{\left(n_{f}\right)}(\mu)}{\pi}\right)^{3}\left[-\frac{4271255}{62208}-\frac{2}{3} \zeta_{2}\left(1+\frac{\ln 2}{3}\right)+\frac{1306661}{13824} \zeta_{3}\right. \\
& -\frac{4937}{864} \ln \frac{\mu^{2}}{M_{h}^{2}}+\frac{385}{144} \ln ^{2} \frac{\mu^{2}}{M_{h}^{2}}-\frac{1}{216} \ln ^{3} \frac{\mu^{2}}{M_{h}^{2}} \\
& +n_{l}\left(\frac{181127}{62208}+\frac{1}{9} \zeta_{2}-\frac{110779}{13824} \zeta_{3}+\frac{109}{48} \ln \frac{\mu^{2}}{M_{h}^{2}}+\frac{53}{96} \ln ^{2} \frac{\mu^{2}}{M_{h}^{2}}\right) \\
& \left.\left.+n_{l}^{2}\left(-\frac{6865}{31104}+\frac{77}{1728} \ln \frac{\mu^{2}}{M_{h}^{2}}-\frac{1}{18} \ln ^{2} \frac{\mu^{2}}{M_{h}^{2}}\right)\right]\right\} \\
& \approx-\frac{1}{12} \frac{\alpha_{s}^{\left(n_{f}\right)}\left(M_{h}\right)}{\pi}\left[1+2.7500 \frac{\alpha_{s}^{\left(n_{f}\right)}\left(M_{h}\right)}{\pi}+\left(9.3507-0.6979 n_{l}\right)\left(\frac{\alpha_{s}^{\left(n_{f}\right)}\left(M_{h}\right)}{\pi}\right)^{2}\right. \\
& \left.+\left(43.6090-6.5383 n_{l}-0.2207 n_{l}^{2}\right)\left(\frac{\alpha_{s}^{\left(n_{f}\right)}\left(M_{h}\right)}{\pi}\right)^{3}\right] \\
& C_{2}^{\mathrm{OS}}=1+\left(\frac{\alpha_{s}^{\left(n_{f}\right)}(\mu)}{\pi}\right)^{2}\left(\frac{5}{18}-\frac{1}{3} \ln \frac{\mu^{2}}{M_{h}^{2}}\right) \\
& +\left(\frac{\alpha_{s}^{\left(n_{f}\right)}(\mu)}{\pi}\right)^{3}\left[-\frac{841}{1296}+\frac{5}{3} \zeta_{3}-\frac{247}{108} \ln \frac{\mu^{2}}{M_{h}^{2}}-\frac{29}{36} \ln ^{2} \frac{\mu^{2}}{M_{h}^{2}}\right. \\
& \left.+n_{l}\left(\frac{53}{216}+\frac{1}{18} \ln ^{2} \frac{\mu^{2}}{M_{h}^{2}}\right)\right]
\end{aligned}
$$




$$
\begin{aligned}
& +\left(\frac{\alpha_{s}^{\left(n_{f}\right)}(\mu)}{\pi}\right)^{4}\left[\frac{578975}{186624}-\frac{4}{3} \zeta_{2}\left(1+\frac{\ln 2}{3}\right)+\frac{374797}{13824} \zeta_{3}-\frac{155}{6} \zeta_{4}\right. \\
& -\frac{575}{36} \zeta_{5}+\frac{31}{72} B_{4}+\left(-\frac{83405}{2592}+\frac{155}{12} \zeta_{3}\right) \ln \frac{\mu^{2}}{M_{h}^{2}}-\frac{2101}{216} \ln ^{2} \frac{\mu^{2}}{M_{h}^{2}} \\
& -\frac{301}{144} \ln ^{3} \frac{\mu^{2}}{M_{h}^{2}}+n_{l}\left(-\frac{11557}{15552}+\frac{2}{9} \zeta_{2}-\frac{221}{288} \zeta_{3}+\frac{25}{12} \zeta_{4}-\frac{1}{36} B_{4}\right. \\
& \left.+\frac{9217}{2592} \ln \frac{\mu^{2}}{M_{h}^{2}}+\frac{109}{144} \ln ^{2} \frac{\mu^{2}}{M_{h}^{2}}+\frac{5}{18} \ln ^{3} \frac{\mu^{2}}{M_{h}^{2}}\right) \\
& \left.+n_{l}^{2}\left(\frac{3401}{23328}-\frac{7}{54} \zeta_{3}-\frac{31}{324} \ln \frac{\mu^{2}}{M_{h}^{2}}-\frac{1}{108} \ln ^{3} \frac{\mu^{2}}{M_{h}^{2}}\right)\right] \\
& 1+0.2778\left(\frac{\alpha_{s}^{\left(n_{f}\right)}\left(M_{h}\right)}{\pi}\right)^{2}+\left(1.3545+0.2454 n_{l}\right)\left(\frac{\alpha_{s}^{\left(n_{f}\right)}\left(M_{h}\right)}{\pi}\right)^{3} \\
& +\left(-12.2884+1.0038 n_{l}-0.0100 n_{l}^{2}\right)\left(\frac{\alpha_{s}^{\left(n_{f}\right)}\left(M_{h}\right)}{\pi}\right)^{4},
\end{aligned}
$$

where we have put $\mu=M_{h}$ in the numerical evaluations. In [59] the leading Yukawa corrections of $\mathcal{O}\left(\alpha_{s}^{n} G_{F} m_{t}^{2}\right)(n=0,1,2)$ to the coefficient functions have been evaluated in the SM. The analytical results are listed in Appendix B.

With the knowledge of $C_{1}$ and $C_{2}$ the construction of the effective Lagrangian is completed. In Section 4.1.4 it will be used in order to compute the hadronic decay rate of the Higgs boson.

Recently the effective Lagrangian has been used in order to consider the Higgs boson production process via gluon fusion. In Refs. [80, 81, 82] a first step to the next-tonext-to-leading order QCD corrections has been done. At this accuracy it is necessary to compute two-loop virtual corrections [80] to the process $g g \rightarrow H$ using the effective $g g H$ vertex of Eq. (4.16). Thus the coefficient function $C_{1}$ enters as a multiplicative constant.

For completeness we want to mention that the low-energy theorem derived in this section has also been specified to the $\gamma \gamma H$ coupling. The analytical expressions can be found in Ref. [49].

\subsection{3 $H \rightarrow g g$ in the background field method}

An interesting alternative to the considerations of the Sections 4.1 .1 and 4.1 .2 is based on the Background Field Method (BFM) [83, 69, 84, 85, 86]. In this framework the gluon field is decomposed into a quantum and a background part where the former only appears as a virtual particle inside the loops. On the contrary, the background field serves as an external field in the Green functions. In the BFM the gauge invariance is maintained while quantizing the theory. This was one of the main motivations for its development.

A comprehensive discussion for the case of QCD and the computation of the two-loop $\beta$ function as a practical application of the BFM can be found in [84. In particular, it is 
shown, that the relation between the charge renormalization constant, $Z_{g}$, (cf. Eq. (3.3)) and the wave function renormalization constant of the background gluon, $Z_{3}^{B}$, reads

$$
Z_{3}^{B}=\frac{1}{Z_{g}^{2}}
$$

Thus, only the background gluon self energy has to be computed in order to obtain the $\beta$ function whereas in the conventional approach also vertex functions have to be considered. E.g., next to the gluon propagator also the ghost two-point function and the gluon-ghost vertex have to be computed. The price one has to pay for these kind of simplifications are more complicated Feynman rules for those vertices involving the background gluon 84. In particular, there are three- and four-gluon vertices which contain additional terms proportional to $1 /(1-\xi)$ where $\xi$ is the gauge parameter defined in Eq. (3.2). As a consequence, it is not possible to choose Landau gauge, which corresponds to $\xi=1$ and which would avoid the renormalization of $\xi$, from the very beginning of the calculation. Rather one has to perform the calculation using a general gauge parameter and either adopt Landau gauge at the end of the calculation or renormalize the gauge parameter.

Concerning the decoupling properties of the background field we can essentially take over the discussion of Section 3. In analogy to Eq. (3.10) one obtains for the decoupling constant of the background gluon field

$$
\zeta_{3}^{B 0}=1+\Pi_{G^{B}}^{0, h}(0)
$$

where $\Pi_{G^{B}}^{0, h}$ is the hard contribution of the transversal part of the bare background gluon polarization function. $\zeta_{3}^{B 0}$ coincides with $\zeta_{3}^{0}$ at one-loop order as only the diagram with a heavy quark loop contributes and the coupling of fermions to background gluons is identical to the one of quantum gluons. Starting from two loops, however, virtual quantum gluons appear inside the Feynman diagrams and the analytical expressions are different. In Fig. 4.3 some typical one-, two- and three-loop diagrams contributing to $\Pi_{G^{B}}^{0, h}(0)$ are shown. One arrives at a finite expression for the decoupling constant with the help of

$$
\zeta_{3}^{B}=\frac{Z_{3}^{B} \zeta_{3}^{B 0}}{Z_{3}^{B \prime}}
$$

It is not surprising that one has the following connection between $\zeta_{3}^{B}$ and $\zeta_{g}$

$$
\zeta_{3}^{B}=\frac{1}{\zeta_{g}^{2}}
$$

which is in analogy to Eq. (4.41). We explicitly checked this relation and computed $\Pi_{G^{B}}^{0, h}(0)$ at three-loop order using a general gauge parameter. After renormalization one obtains

$$
\zeta_{3}^{B, \mathrm{OS}}=1+\frac{\alpha_{s}^{\left(n_{l}\right)}(\mu)}{\pi}\left(\frac{1}{6} \ln \frac{\mu^{2}}{M_{h}^{2}}\right)+\left(\frac{\alpha_{s}^{\left(n_{l}\right)}(\mu)}{\pi}\right)^{2}\left(\frac{7}{24}+\frac{19}{24} \ln \frac{\mu^{2}}{M_{h}^{2}}+\frac{1}{36} \ln ^{2} \frac{\mu^{2}}{M_{h}^{2}}\right)
$$



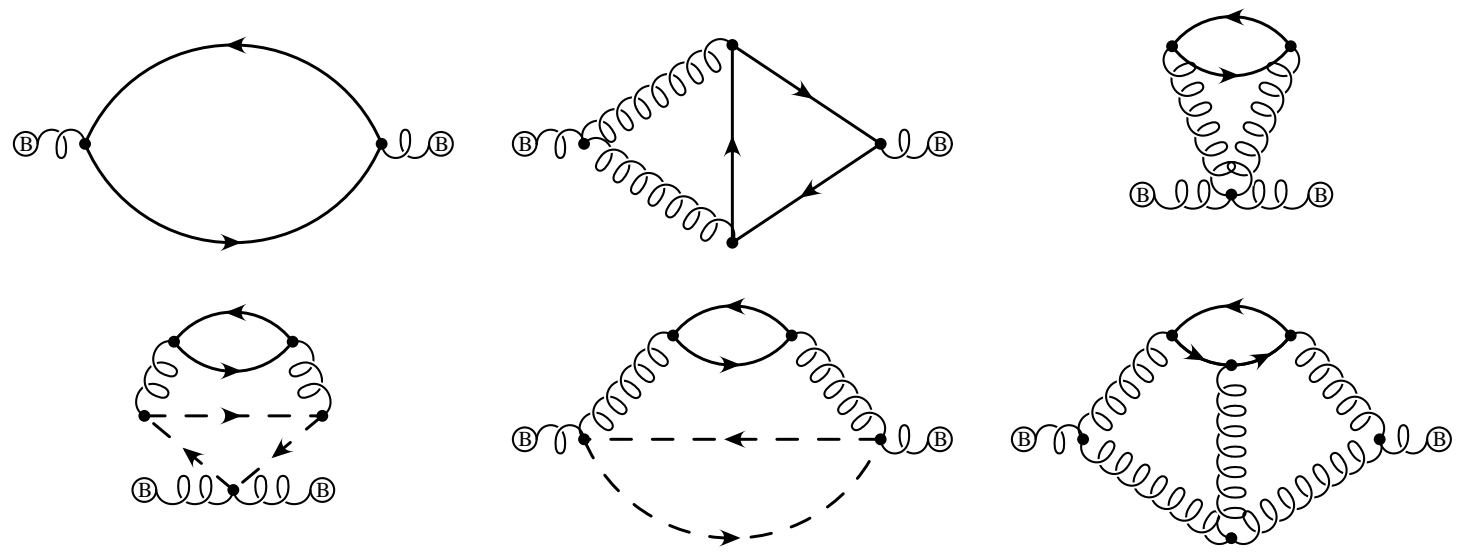

Figure 4.3: Typical Feynman diagrams contributing to the two-point function with external background gluons. Looped, solid, and dashed lines represent gluons, heavy quarks, and ghosts, respectively.

$$
\begin{aligned}
& +\left(\frac{\alpha_{s}^{\left(n_{l}\right)}(\mu)}{\pi}\right)^{3}\left[\frac{58933}{124416}+\frac{2}{3} \zeta_{2}\left(1+\frac{1}{3} \ln 2\right)+\frac{80507}{27648} \zeta_{3}+\frac{8941}{1728} \ln \frac{\mu^{2}}{M_{h}^{2}}\right. \\
& \left.+\frac{511}{576} \ln ^{2} \frac{\mu^{2}}{M_{h}^{2}}+\frac{1}{216} \ln ^{3} \frac{\mu^{2}}{M_{h}^{2}}+n_{l}\left(-\frac{2479}{31104}-\frac{\zeta_{2}}{9}-\frac{409}{1728} \ln \frac{\mu^{2}}{M_{h}^{2}}\right)\right]
\end{aligned}
$$

which is in agreement with Eq. (3.30). The 219 diagrams contributing to $\Pi_{G^{B}}^{0, h}(0)$ have to be compared with the $189+25+228=442$ diagrams which are necessary in order to obtain the result of Eq. (3.30).

Eq. (4.44) also significantly simplifies the computation of the coefficient function $C_{1}$. Due to Eq. (4.33) it is simply obtained via

$$
C_{1}=2 \partial_{h} \ln \zeta_{3}^{B}
$$

This coincides with the naive expectation that the effective coupling of the Higgs boson is generated by taking derivatives of the gluon polarization function with respect to the heavy quark mass. Furthermore Eq. (4.46) agrees with the low-energy theorem for the photon-Higgs interaction as derived in [49]. In this sense we could claim that the background gluon is "more physical" than the gluon in the conventional approach.

In this context we want to refer to 87] where low-energy theorems in Higgs physics have been considered at one- and two-loop order. In particular it was realized that the decay rate of a scalar Higgs boson into two photons can be obtained by naive differentiation of the photon self energy if the latter is computed in the framework of the pinch technique.

As a further check on the result for $C_{1}$ we also perform the direct calculation by considering the quantity $\Gamma_{G^{B} G^{B} \mathcal{O}_{h}}^{\mu \nu}\left(p_{1}, p_{2}\right)$ which is defined in analogy to Eq. (4.22):

$$
\delta^{a b} \Gamma_{G^{B} G^{B} \mathcal{O}_{h}}^{0, \mu \nu}\left(p_{1}, p_{2}\right)=i^{2} \int \mathrm{d} x \mathrm{~d} y e^{i\left(p_{1} \cdot x+p_{2} \cdot y\right)}\left\langle T G^{B, 0, a \mu}(x) G^{B, 0, b \nu}(y) \mathcal{O}_{h}(0)\right\rangle^{1 \mathrm{PI}}
$$


The only difference is the presence of external background (instead of quantum) fields. We evaluated the 732 vertex diagrams in the spirit of Eq. (4.30) and could confirm the order $\alpha_{s}^{3}$ terms in Eqs. (4.37) and (4.39).

Due to the fact that there are no diagrams involving external ghost fields contributing to Eq. (4.46) there is no admixture from $\mathcal{O}_{4}^{0}$ while matching $\Gamma_{G^{B} G^{B} \mathcal{O}_{h}}^{\mu \nu}\left(p_{1}, p_{2}\right)$ with the effective theory even for the choice $p_{1}=-p_{2}$. Thus we can immediately set $p_{1}=-p_{2}=p$ in Eq. (4.47) and consider the limit $p \rightarrow 0$. No complicated projector like in Eq. (4.30) is necessary which simplifies the evaluation of the diagrams with external background gluons.

\subsubsection{Hadronic decay rate of the SM Higgs boson}

In the following we present the current status of the total decay rate of the SM Higgs boson ${ }^{[3]}$ in the intermediate mass range. This is done on the basis of the effective Lagrangian Eq. (4.16), where the top quark takes over the role of the heavy quark $h$.

The total decay rate into hadrons can be cast in the form[

$$
\begin{aligned}
\Gamma(H \rightarrow \text { hadrons })= & \left(1+\delta_{u}\right)^{2}\left\{\sum_{q} A_{q \bar{q}}\left[\left(1+\Delta_{22}^{q}\right)\left(C_{2 q}\right)^{2}+\Delta_{12}^{q} C_{1} C_{2 q}\right]\right. \\
& \left.+A_{g g} \Delta_{11}\left(C_{1}\right)^{2}+A_{g g} \Delta_{g}^{\text {hdo }}+\sum_{q} A_{q \bar{q}}\left[\Delta_{q}^{\text {hdo }}+\left.\Delta_{q}^{\text {weak }}\right|_{x_{t}=0}\right]\right\},
\end{aligned}
$$

with $A_{q \bar{q}}=3 G_{F} M_{H} m_{q}^{2} /(4 \pi \sqrt{2})$ and $A_{g g}=4 G_{F} M_{H}^{3} /(\pi \sqrt{2})$. The terms in the first line and the first term in the second line proportional to $A_{g g}$ have their origin in Eq. (4.16). In particular, the universal corrections $\delta_{u}$ arise from the renormalization of the factor $H^{0} / v^{0}$ and are known to order $\alpha_{s}^{2} G_{F} m_{t}^{2}$ [77]. The factors $\Delta_{i j}$ contain the QED and QCD corrections from the light degrees of freedom only, while the terms $\Delta^{\text {hdo }}$ summarize the corrections coming from higher dimensional operators. They are at least suppressed by a factor $\alpha_{s}^{2} M_{H}^{2} / M_{t}^{2}$. In Eq. (4.48) we separately display the weak contribution where the leading term of order $G_{F} m_{t}^{2}$ is stripped off. It is denoted by $\left.{ }^{\text {可 }} \Delta_{q}^{\text {weak }}\right|_{x_{t}=0}$.

Typical diagrams contributing to the QCD corrections of $\Delta_{22}^{q}$ are pictured in Fig. 4.4. At one-loop order they have been evaluated in [90, 91] keeping the full dependence of the quark mass. The dominant corrections at order $\mathcal{O}\left(\alpha_{s}^{2}\right)$, i.e. those obtained keeping only the factor $m_{q}^{2}$ from the Yukawa coupling, have been evaluated in 92]. The calculation has later on been improved and the correction terms proportional to $m_{q}^{2} / M_{H}^{2}$ became available [93, 94]. A naive expansion in $m_{q}$ is sufficient for their computation. Beyond the quadratic term, however, one either has to adopt the method discussed in Section 4.2 or apply the large-momentum expansion. The latter has been performed in 950 and

\footnotetext{
${ }^{13}$ For review articles we refer to 888,89 .

${ }^{14}$ The additional index "q" for the coefficient function $C_{2}$ indicates that there might be an explicit dependence on the flavour through elektroweak corrections.

${ }^{15}$ For the definition of $x_{t}$ see Eq. (B.7)
} 


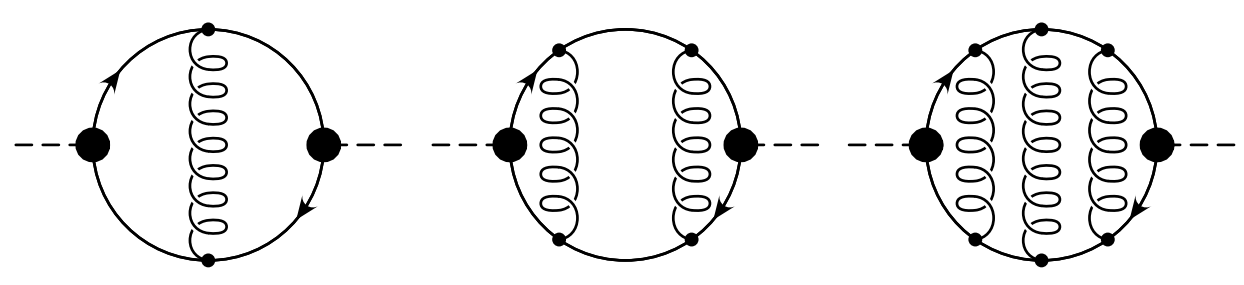

Figure 4.4: Typical Feynman diagrams contributing to $\Delta_{22}^{q}$. The solid circles represent the operator $\mathcal{O}_{2}$.

mass correction terms up to order $\left(m_{b}^{2} / M_{H}^{2}\right)^{8}$ have been evaluated. For a Higgs boson in the intermediate-mass range considered in this Subsection the corrections beyond the quadratic term are quite small and can safely be neglected. In 95] the higher order terms have been considered in the context of a heavy Higgs boson which can also decay into top quarks. In this case it turned out that even the quartic terms are important and only an expansion up to order $\left(m_{t}^{2} / M_{H}^{2}\right)^{8}$ gives satisfactory results. In Section 5.2 we review the calculation and numerical results are presented in Tab. 5.1. For completeness we want to mention that the imaginary part of the correlator $\left\langle\mathcal{O}_{2} \mathcal{O}_{2}\right\rangle$ has even been evaluated at four-loop order using the technique described in Section 2.2. This leads to corrections of order $\alpha_{s}^{3}$ 40].

Next to the pure QCD corrections there are also the contributions of order $\alpha$ and the mixed QED/QCD terms of order $\alpha \alpha_{s}$ [96, 97] which can easily be extracted form the QCD results. In summary, the numerical result for the discussed terms read

$$
\begin{aligned}
\Delta_{22}^{q}\left(M_{H}\right)= & \frac{\alpha_{s}^{(5)}\left(M_{H}\right)}{\pi}\left[5.66667+\left(35.9400-1.3587 n_{l}\right) \frac{\alpha_{s}^{(5)}\left(M_{H}\right)}{\pi}\right. \\
& \left.+\left(164.1392-25.7712 n_{l}+0.2590 n_{l}^{2}\right)\left(\frac{\alpha_{s}^{(5)}\left(M_{H}\right)}{\pi}\right)^{2}\right] \\
& +\frac{\bar{\alpha}\left(M_{H}\right)}{\pi} Q_{q}^{2}\left(4.2500+11.7097 \frac{\alpha_{s}^{(5)}\left(M_{H}\right)}{\pi}\right)
\end{aligned}
$$

where $n_{l}$ is the number of light quarks.

The imaginary part of the mixed correlator $\left\langle\mathcal{O}_{1} \mathcal{O}_{2}\right\rangle$ is denoted by $\Delta_{12}^{q}$. Next to contributions to the partial width into quarks it also involves purely gluonic final states as can be seen from the diagrams shown in Fig. 4.5. The contributions at two- and three-loop order have been computed in [94, 98, 75] and [99], respectively. In numerical form $\Delta_{12}^{q}$ is given by

$$
\Delta_{12}^{q}\left(M_{H}\right)=\frac{\alpha_{s}^{(5)}\left(M_{H}\right)}{\pi}\left[-30.667+\left(-524.853+20.647 n_{l}\right) \frac{\alpha_{s}^{(5)}\left(M_{H}\right)}{\pi}\right] .
$$

In case one is only interested in final states involving quarks the purely gluonic cuts have to be subtracted. Currently they are only known at order $\alpha_{s}$. To this order the subtracted 


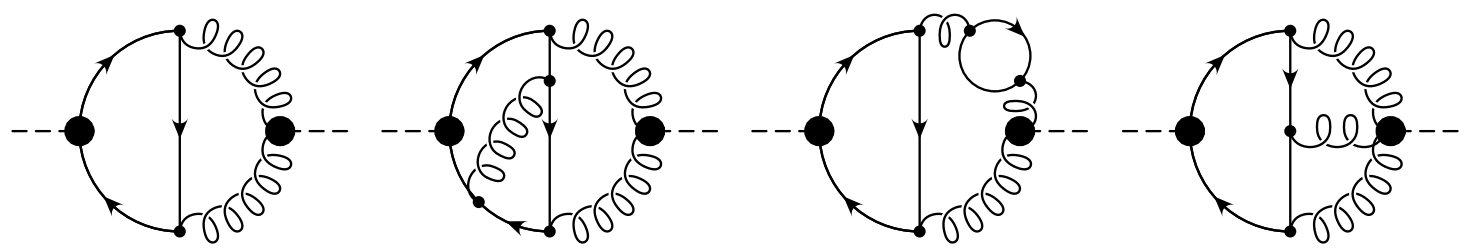

Figure 4.5: Two- and some of the three-loop diagrams contributing to $\Delta_{12}^{q}$. The solid circles represent the operators $\mathcal{O}_{1}$ and $\mathcal{O}_{2}$, respectively.

result reads 75]

$$
\Delta_{12}^{q^{\prime}}\left(M_{H}\right)=\frac{\alpha_{s}^{(5)}\left(M_{H}\right)}{\pi}\left[-\frac{76}{3}+8 \zeta_{2}-\frac{4}{3} \ln ^{2} \frac{m_{q}^{2}}{M_{H}^{2}}-8 \ln \frac{\mu^{2}}{M_{H}^{2}}\right]+\ldots,
$$

where the ellipses indicate terms of order $\alpha_{s}^{2}$. Note the logarithmic singularity in the light-quark mass which arises from the fact that only parts of the final state are contained in Eq. (4.51).

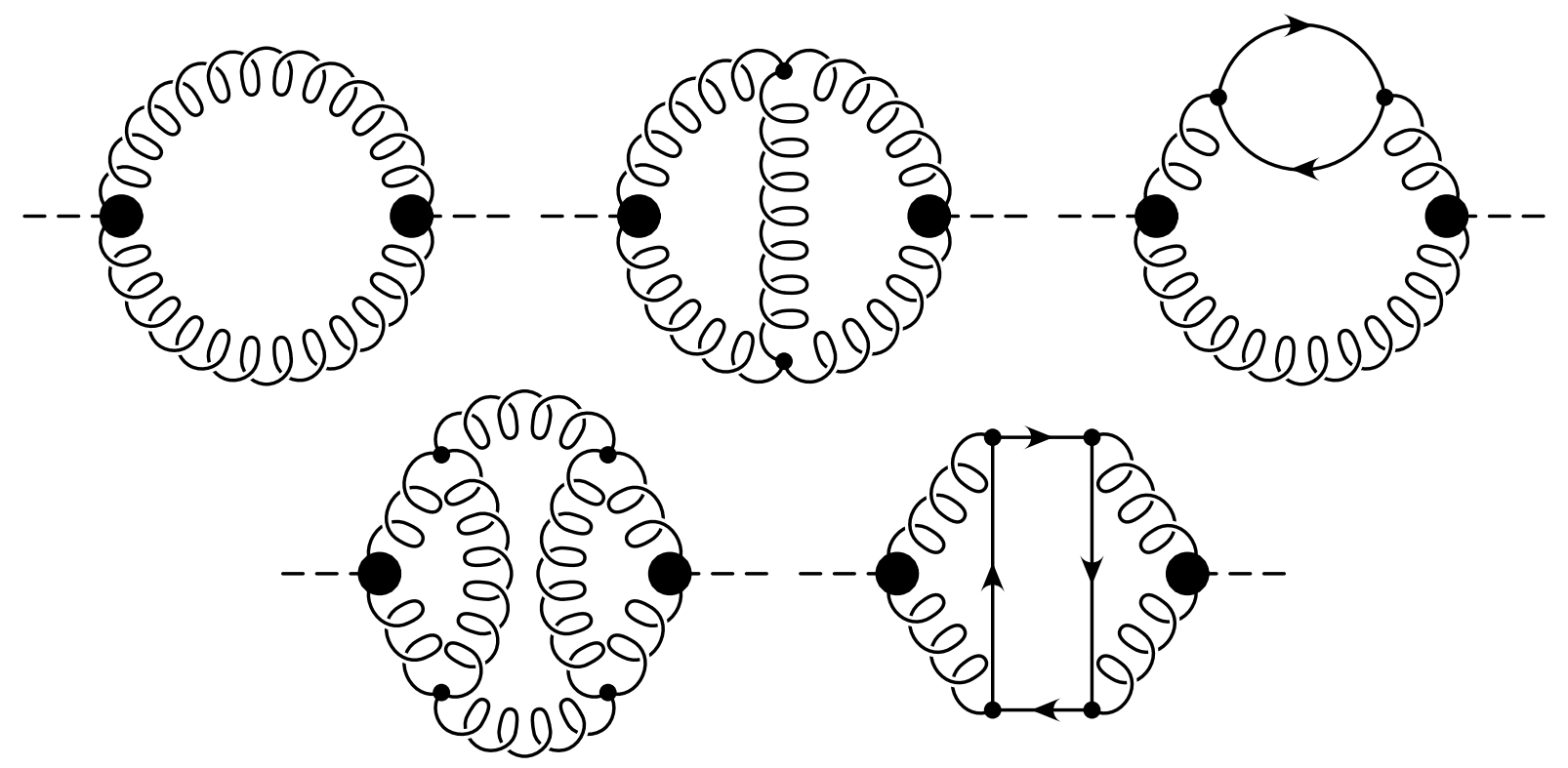

Figure 4.6: Typical Feynman diagrams contributing to the correlator $\left\langle\mathcal{O}_{1} \mathcal{O}_{1}\right\rangle$. Looped, solid, and dashed lines represent gluons, light quarks, and Higgs bosons, respectively. Solid circles represent insertions of $\mathcal{O}_{1}$.

The correlator formed by the operator $\mathcal{O}_{1}$ mainly contains cuts arising from gluons. However, starting from two loops there are also contributions from light quarks and at 
order $\alpha_{s}^{2}$ there are even cuts involving no gluons at all (cf. second diagram in the lower row of Fig. 4.6). In Fig. 4.6 some typical diagrams are pictured. In particular, the combination $\left(C_{1}\right)^{2} \Delta_{11}$ contains the contribution from all the diagrams pictured in Figure 4.7. The twoloop contribution has been evaluated in 100, 101 and the order $\alpha_{s}^{2}$ terms can be found if [79]. If we set $\mu^{2}=M_{H}^{2}$ and evaluate the correlator for $q^{2}=M_{H}^{2}$ we obtain

$$
\begin{aligned}
\Delta_{11}\left(M_{H}^{2}\right)= & 1+\frac{\alpha_{s}^{(5)}\left(M_{H}\right)}{\pi}\left(18.250-1.167 n_{l}\right) \\
& +\left(\frac{\alpha_{s}^{(5)}\left(M_{H}\right)}{\pi}\right)^{2}\left(242.973-39.374 n_{l}+0.902 n_{l}^{2}\right) .
\end{aligned}
$$

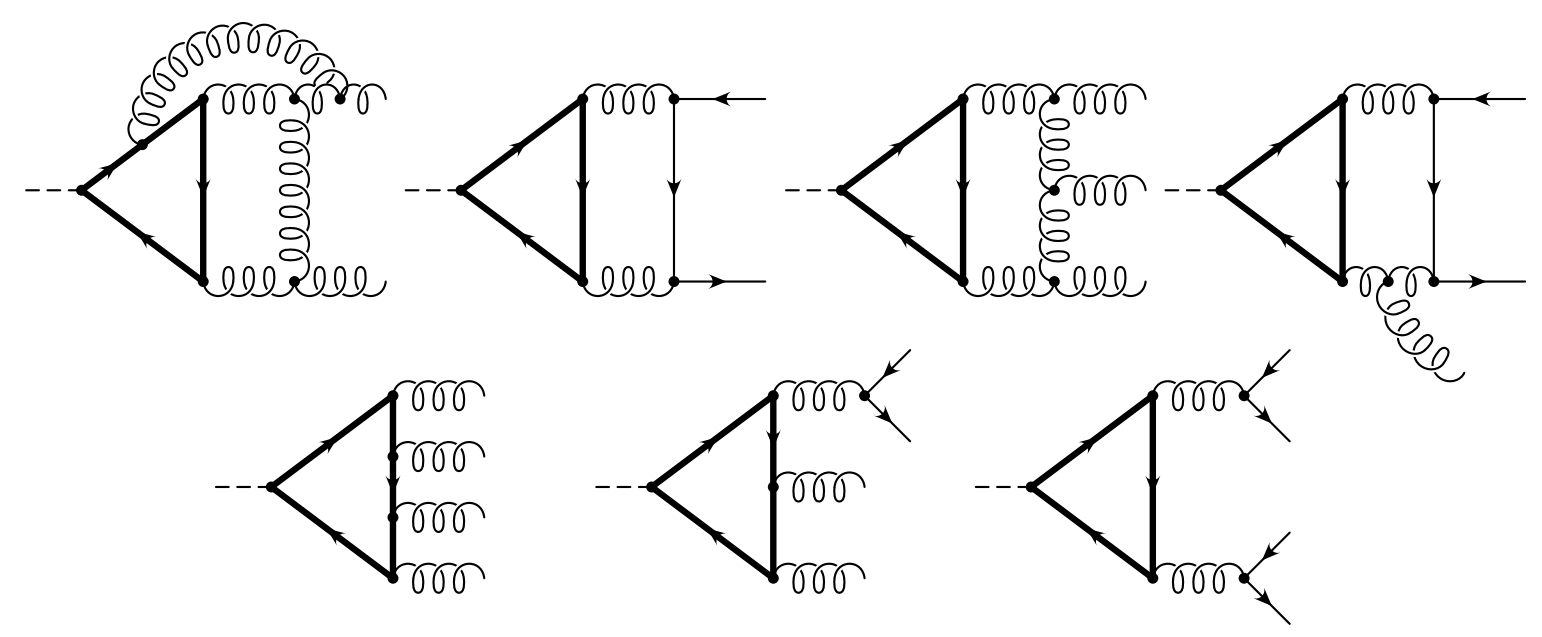

Figure 4.7: Typical diagrams generating $\mathcal{O}\left(\alpha_{s}^{2}\right)$ corrections to $\Gamma(H \rightarrow g g)$. Bold-faced (dashed) lines represent the top quark (Higgs boson).

The contributions to $\Delta^{\text {hdo }}$ are not covered by Eq. (4.16). In the language of the effective Lagrangian it would require to deal with operators of dimension six and higher. However, up to now they have not been studied in detail. The approach adopted in Refs. [94, 98] is based on asymptotic expansion which is applied to the propagator-type diagrams involving a top-quark loop. At order $\alpha_{s}^{2}$ there are two classes of such diagrams contributing to $\Delta_{q}^{\text {hdo }}$, namely the double-triangle (or singlet) and the double bubble diagrams. The exact result for the imaginary part of the latter with massless external quark lines and heavy virtual top quark can be found in [102. The leading term contribution to $\Delta_{q}^{\text {hdo }}$ reads

$$
\begin{aligned}
\Delta_{q}^{\text {hdo }} & =\left(\frac{\alpha_{s}^{(5)}}{\pi}\right)^{2} \frac{M_{H}^{2}}{M_{t}^{2}}\left(\frac{5863}{24300}-\frac{113}{1620} \ln \frac{M_{H}^{2}}{M_{t}^{2}}\right) \\
& =\left(\frac{\alpha_{s}^{(5)}}{\pi}\right)^{2} \frac{M_{H}^{2}}{M_{t}^{2}}\left(0.241-0.070 \ln \frac{M_{H}^{2}}{M_{t}^{2}}\right)
\end{aligned}
$$


The higher order contributions in $M_{H}^{2} / M_{t}^{2}$ are very small and can safely be neglected even for $M_{H}=M_{t}$. For $\Delta_{g}^{\text {hdo }}$ one obtains [98]

$$
\begin{aligned}
\Delta_{g}^{\text {hdo }} & =\left(\frac{\alpha_{s}^{(5)}}{\pi}\right)^{2} \frac{M_{H}^{2}}{M_{t}^{2}} \frac{7}{60}+\left(\frac{\alpha_{s}^{(5)}}{\pi}\right)^{3} \frac{M_{H}^{2}}{M_{t}^{2}}\left(\frac{2249}{1080}-\frac{7}{30} \ln \frac{M_{H}^{2}}{M_{t}^{2}}\right) \\
& =\left(\frac{\alpha_{s}^{(5)}}{\pi}\right)^{2} \frac{M_{H}^{2}}{M_{t}^{2}} 0.11667+\left(\frac{\alpha_{s}^{(5)}}{\pi}\right)^{3} \frac{M_{H}^{2}}{M_{t}^{2}}\left(2.0824-0.23333 \ln \frac{M_{H}^{2}}{M_{t}^{2}}\right)
\end{aligned}
$$

where again the higher order terms in $M_{H}^{2} / M_{t}^{2}$ are much smaller. The contribution to $\Delta_{g}^{\text {hdo }}$ is obtained from the application of the hard-mass procedure to the propagatortype diagrams where the external Higgs bosons are attached to top quarks and they subsequently couple to gluons. To leading order in the expansion for large $m_{t}$ one obtains the one- and two-loop diagrams of Fig. 4.6. The comparison of Eq. (4.54) with Eq. (4.52) shows that also in this case the contributions from the higher dimensional operators are small. We want to mention that for $H \rightarrow g g$ a numerical calculation of order $\alpha_{s}^{2}$ exists which takes the complete mass dependence into account [101].

At one-loop the complete weak corrections have been computed in analytical from [103, 104. If we put $m_{q}=0$ and consider the limit $M_{H} \ll 2 M_{W},\left.\Delta_{q}^{\text {weak }}\right|_{x_{t}=0}$ takes the form [104]

$$
\left.\Delta_{q}^{\text {weak }}\right|_{x_{t}=0}=\frac{G_{F} M_{Z}^{2}}{8 \pi^{2} \sqrt{2}}\left[\frac{1}{2}-3\left(1-4 s_{w}^{2}\left|Q_{q}\right|\right)^{2}+c_{w}^{2}\left(\frac{3}{s_{w}^{2}} \ln c_{w}^{2}-5\right)\right] .
$$

The leading $m_{t}^{2}$ term is stripped off as it is already contained in the universal factor $\delta_{u}$. Expressed in terms of the $\overline{\mathrm{MS}}$ top quark mass the latter reads in numerical form 105, 77]

$$
\begin{aligned}
\delta_{u}= & \frac{7}{6} N_{c} x_{t}\left[1+\frac{\alpha_{s}^{(6)}(\mu)}{\pi}\left(2 \ln \frac{\mu^{2}}{m_{t}^{2}}+0.869561\right)\right. \\
& \left.+\left(\frac{\alpha_{s}^{(6)}(\mu)}{\pi}\right)^{2}\left(3.750000 \ln ^{2} \frac{\mu^{2}}{m_{t}^{2}}+6.010856 \ln \frac{\mu^{2}}{m_{t}^{2}}-2.742226\right)\right] .
\end{aligned}
$$

We want to mention that also non-universal radiative corrections to $C_{1}$ and $C_{2 q}$, which are enhanced by a factor $G_{F} m_{t}^{2}$, are available up to the three-loop order [77, 75, 59]. They will be listed below in comparison with the terms of $\mathcal{O}\left(\alpha_{s}^{3}\right)$.

At this point we want to compare the relative size of the individual terms. In particular we have in mind terms of order $\mathcal{O}\left(\alpha_{s}^{3}\right), \alpha_{s}^{2} m_{b}^{2} / M_{H}^{2}, \alpha \alpha_{s}, \alpha_{s}^{2} X_{t}$ and $\alpha_{s}^{2} M_{H}^{2} / M_{t}^{2}$. For this purpose we will consider all quarks with mass lighter than $m_{b}$ as massless. This means that the sum in Eq. (4.48) reduces to $q=b$ which is conveniently written in the form

$$
\Gamma(H \rightarrow \text { hadrons })=A_{b \bar{b}}\left(1+\Delta_{l}^{b}+\Delta_{t}\right)+\frac{A_{g g}}{144}\left(\frac{\alpha_{s}^{(5)}}{\pi}\right)^{2} \Delta_{g},
$$

where $\Delta_{l}^{b}$ contains only corrections from light degrees of freedom. All top-induced terms proportional to $A_{b \bar{b}}$ from Eq. (4.48) are contained in $\Delta_{t}$, which we express in terms of 
$\alpha_{s}^{(5)}(\mu) . \Delta_{g}$ contains the corrections from the gluonic final state. Choosing $\mu^{2}=M_{H}^{2}$ and $n_{l}=5$ we find

$$
\begin{aligned}
\Delta_{l}^{b}= & -6 \frac{\left(m_{b}^{(5)}\right)^{2}}{M_{H}^{2}}+0.472 \frac{\bar{\alpha}\left(M_{H}\right)}{\pi}+1.301 \frac{\bar{\alpha}\left(M_{H}\right)}{\pi} a_{H}^{(5)}+a_{H}^{(5)}\left(5.667-40.000 \frac{\left(m_{b}^{(5)}\right)^{2}}{M_{H}^{2}}\right) \\
& +\left(a_{H}^{(5)}\right)^{2}\left(29.147-87.725 \frac{\left(m_{b}^{(5)}\right)^{2}}{M_{H}^{2}}\right)+41.758\left(a_{H}^{(5)}\right)^{3} \\
\Delta_{t}= & \left(a_{H}^{(5)}\right)^{2}\left[3.111-0.667 L_{t}+\frac{\left(m_{b}^{(5)}\right)^{2}}{M_{H}^{2}}\left(-10+4 L_{t}+\frac{4}{3} \ln \frac{\left(m_{b}^{(5)}\right)^{2}}{M_{H}^{2}}\right)\right] \\
& +\left(a_{H}^{(5)}\right)^{3}\left(50.474-8.167 L_{t}-1.278 L_{t}^{2}\right)+\left(a_{H}^{(5)}\right)^{2} \frac{M_{H}^{2}}{M_{t}^{2}}\left(0.241-0.070 L_{t}\right) \\
& +X_{t}\left[1-4.913 a_{H}^{(5)}+\left(a_{H}^{(5)}\right)^{2}\left(-72.117-20.945 L_{t}\right)\right] \\
\Delta_{g}= & 1+X_{t}+a_{H}^{(5)}\left[17.917+30.3369 X_{t}\right]+\left(a_{H}^{(5)}\right)^{2}\left[156.808+5.708 \ln \frac{M_{H}^{2}}{m_{t}^{2}}\right]
\end{aligned}
$$

with $a_{H}^{(5)}=\alpha_{s}^{(5)}\left(M_{H}\right) / \pi, L_{t}=\ln M_{H}^{2} / M_{t}^{2}$, and $X_{t}=G_{F} M_{t}^{2} /\left(8 \pi^{2} \sqrt{2}\right)$. In Eqs. 4.58) and (4.59) also the quadratic mass correction terms are listed. In $\Delta_{l}$ they are obtained from the naive expansion of the diagrams. The $\left(m_{b}^{(5)}\right)^{2} / M_{H}^{2}$ corrections in $\Delta_{t}$ arise from the singlet diagram with one top and one bottom quark triangle. In this case a naive expansion fails as can be seen by the logarithmic term in Eq. (4.59). Instead the asymptotic expansion has to be applied [94]. Both for $\Delta_{l}$ and $\Delta_{t}$ one observes that the $\mathcal{O}\left(\alpha_{s}^{3}\right)$ term proves to be numerically more important than the power suppressed contribution of $\mathcal{O}\left(\alpha_{s}^{2} m_{b}^{2} / M_{H}^{2}\right)$. Note, that Eq. (4.59) contains contributions with pure gluonic final states which is due to diagrams of the type in Fig. 4.5.

In the approximation considered here we have $-2 \lesssim L_{t}<0$. This means that the logarithm needs not necessarily to be re-summed as in addition the coefficients in front of $L_{t}$ are much smaller than the constant term.

A comparison of Eqs. (4.58) and (4.59) shows that the top-induced corrections in $\Delta_{t}$ of $\mathcal{O}\left(\alpha_{s}^{3}\right)$ are numerically of the same size as the ones arising from "pure" QCD. Furthermore one should mention that the coefficient of the $M_{t}$-suppressed terms are tiny and, as $\alpha_{s} / X_{t} \approx 30$, also the $\alpha_{s}^{2} X_{t}$ enhanced terms are less important than the cubic QCD corrections. This is also the case for Eq. (4.60). For comparison in Eq. (4.58) also the two-loop corrections of order $\alpha \alpha_{s}$ are listed. In principle also higher order mass corrections are available 95]. However, in the case of bottom quarks it turns out that they are tiny.

In summary, we have shown that for an intermediate-mass Higgs boson the application of the effective Lagrangian (cf. Eq. (4.13)) is quite successful and enables the computation of the hadronic Higgs decay up to orders $\alpha_{s}^{3}$ and $\alpha_{s}^{4}$ for the quark and gluon final states, respectively. The smallness of the higher dimensional operators (cf. Eq. (4.53) and (4.54)) justifies this approach. In conclusion we can state that the perturbative expansion of the 
hadronic width of the Higgs boson is well under control.

\subsection{Quartic mass corrections to $\sigma\left(e^{+} e^{-} \rightarrow\right.$ hadrons $)$}

The total cross section for hadron production in electron-positron annihilation, $\sigma\left(e^{+} e^{-} \rightarrow\right.$ hadrons), is one of the most fundamental observables in particle physics (for a review see [106]). For energies sufficiently far above threshold it can be predicted by perturbative QCD, and it is well accessible experimentally from threshold up to the high energies of LEP and a future linear collider. It allows for a precise determination of the strong coupling $\alpha_{s}$ and, once precision measurements at different energies are available, for a test of its evolution dictated by the renormalization group equation.

Often the center-of-mass energy is much larger than the quark masses which then can safely be neglected. However, there are also many situations where it is important to take into account the effect of finite quark masses [107]. E.g., one can think of charm or bottom quark production not far above their production thresholds [108, 109], or of top quark production at a future linear collider [110].

The complete mass-dependence at order $\alpha_{s}$ to $R(s) \equiv \sigma\left(e^{+} e^{-} \rightarrow\right.$ hadrons $) / \sigma\left(e^{+} e^{-} \rightarrow\right.$ $\mu^{+} \mu^{-}$) has been evaluated quite some time ago in analytical form 111 in the context of QED. At order $\alpha_{s}^{2}$ this task already becomes much less trivial. The massless approximation became available quite some time ago [60]. However, only for a certain class of diagrams - the ones containing a second massless quark pair - the full quark mass dependence could be obtained in analytical form using conventional methods [112. For all other contributions different methods have to be applied which are discussed in detail in Section 5. Here, we only want to mention that a crucial ingredient is the application of the large-momentum procedure which provides an expansion in $\mathrm{m}^{2} / \mathrm{s}$. At order $\alpha_{s}^{3}$ also this method fails as it would be necessary to evaluate massless four-loop propagatortype diagrams. At the moment this is not possible. Thus a different strategy has to be employed which we will describe below.

In addition to the massless result, which has been obtained in [39, 38], the $\mathrm{m}^{2} / \mathrm{s}$ terms of $\mathcal{O}\left(\alpha_{s}^{3}\right)$ have been calculated in [113]. They were obtained by reconstructing the logarithmic $\alpha_{s}^{3} m^{2} / s$ terms for the polarization function $\Pi\left(q^{2}\right)$ from the knowledge of the full three loop $\mathcal{O}\left(\alpha_{s}^{2} m^{2} / s\right)$ result of 114 with the help of the renormalization group equations. These are sufficient to calculate the $\mathrm{m}^{2} / \mathrm{s}$ terms of the imaginary part in the time-like region. A generalization of this approach has been formulated for the quartic mass terms in [72, 73 and was originally adopted for the calculation of $\alpha_{s}^{2} m^{4} / s^{2}$ terms [73].

The basic ingredients are the OPE 68 and the renormalization group equations (RGE). The idea is to apply the OPE to the correlator of two currents and compute its imaginary part which immediately leads to corrections for $R(s)$. The current correlator is expressed as a sum over local operators multiplied by coefficient functions which represent the short distance part of the process. Afterwards one exploits the RGEs in order to relate different pieces in the sum and to construct the logarithmic terms of the polarization function. In addition to the anomalous mass dimension and the $\beta$-function, the anomalous dimensions of the operators of dimension four are required in appropriate 


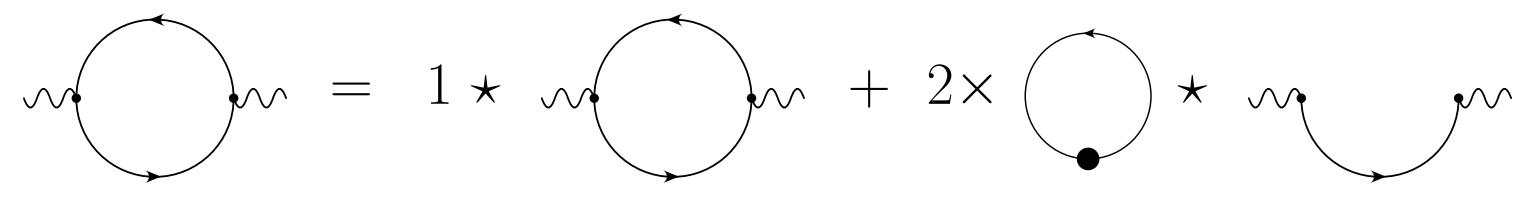

Figure 4.8: Large-momentum procedure for the one-loop photon polarization function. The quark lines carry the mass $m$.

order.

Let us be more specific and consider the time-ordered product of two currents. The application of the OPE leads to

$$
T^{j}(q)=i \int \mathrm{d}^{4} x e^{i q x} \mathrm{~T} j(x) j(0) \stackrel{-q^{2} \rightarrow \infty}{\sim} \sum_{n} C_{n}(q) \mathcal{O}_{n}
$$

where the dependence of the coefficient functions $C_{n}(q)$ on the large scale $q$ is made explicit. In Eq. (4.61) we have to consider all operators $\mathcal{O}_{n}$ of dimension four as exactly those contribute to the quartic mass corrections. They can be found in Eqs. (4.1) and (4.2).

Actually the contributing operators essentially coincide with the ones of Section 4.1. There, however, the top quark mass took over the role of the large scale and the external momentum was supposed to be much smaller. As a consequence the coefficient functions depend on $M_{t}$ and are computed with the help of vacuum integrals. On the contrary, we will see below that the coefficient functions of Eq. (4.61) are expressed through massless propagator-type integrals.

The virtue of Eq. (4.61) becomes obvious if one takes the vacuum expectation value. Then the left-hand side turns into the polarization function which in the following we generically call $\Pi\left(q^{2}\right)$. On the right-hand side we obtain a sum of vacuum expectation values of the local operators multiplied by the coefficient functions. As already mentioned, the latter only depend on $q$ whereas the scale of the vacuum expectation values is given by the quark mass. This strongly resembles the large-momentum procedure. Also there a factorization of the scales is achieved. In fact, it can be shown that for a certain choice of the operator basis an identification of the $C_{n}(q)$ with the hard subgraphs and of the vacuum expectation values with the co-subgraphs is possible.

For illustration we consider the correlator of two vector currents at one-loop order. The application of the large-momentum procedure is visualized in Fig. 4.8. The prescription tells us that the first term on the right-hand side of the equation has to be expanded in the quark mass, $m$, leading to massless integrals. Also the subdiagram on the very right in the second term has to be expanded in $m$ and then has to be inserted in the blob of the loop-diagram. Thus one ends up with a vacuum integral. Taking into account terms up to quartic order one gets ${ }^{16}$ (see, e.g., Ref. [115, 116])

$$
\Pi_{\text {bare }}^{(0)}\left(q^{2}\right) \stackrel{q^{2} \gg m^{2}}{=} \frac{3}{16 \pi^{2}}\left\{\frac{4}{3 \varepsilon}+\frac{20}{9}-\frac{4}{3} l_{q \mu}+8 \frac{m^{2}}{q^{2}}+\left(\frac{m^{2}}{q^{2}}\right)^{2}\left(4+8 l_{q m}\right)+\ldots\right\},
$$

\footnotetext{
${ }^{16}$ For a precise definition of the polarization function see Eqs. (5.15) and (5.20) in Section 5 .
} 
with $l_{q \mu}=\ln \left(-q^{2} / \mu^{2}\right)$ and $l_{q m}=\ln \left(-q^{2} / m^{2}\right)$. The ellipses in Eq. (4.62) denote higher order mass correction terms. On dimensional reasons it is clear that the $m^{0}$ and $m^{2}$ term only comes from the first diagram on the right-hand side of Fig. 4.8. Also the $m^{4} \ln \left(-q^{2}\right)$ term can only arise from this diagram. On the other hand, the massive logarithm, $m^{4} \ln m^{2}$, originates from the vacuum integral of Fig. 4.8 .

Let us now look at the result provided by the OPE. At lowest order in $\alpha_{s}$ only the two operators $\mathcal{O}_{2}$ and $\mathcal{O}_{6}$ of Eq. (4.1) contribute. The product of the corresponding vacuum expectation values and the coefficient functions reads (see, e.g., Ref. [115, 14])

$$
\begin{aligned}
& C_{2}^{0}\left\langle\mathcal{O}_{2}^{0}\right\rangle=\frac{3}{16 \pi^{2}}\left(\frac{m^{2}}{q^{2}}\right)^{2}\left(\frac{8}{\varepsilon}+12+8 l_{\mu m}\right), \\
& C_{6}^{0}\left\langle\mathcal{O}_{6}^{0}\right\rangle=\frac{3}{16 \pi^{2}}\left(\frac{m^{2}}{q^{2}}\right)^{2}\left(-\frac{8}{\varepsilon}-8+8 l_{q \mu}\right) .
\end{aligned}
$$

Thus, $C_{6}^{0}\left\langle\mathcal{O}_{6}^{0}\right\rangle$ exactly repoduces the $m^{4} \ln \left(-q^{2}\right)$ of the massless diagram of Fig. 4.8, whereas $\left\langle\mathcal{O}_{2}^{0}\right\rangle$ provides the massive logarithm. The sum of the two contributions in Eq.(4.63) reproduces the $m^{4}$ terms of Eq. (4.62).

Note that in the above consideration no normal-ordering prescription has been used. Otherwise the vacuum expectation value of the operator $\mathcal{O}_{2}$ would be zero. Furthermore, the coefficient function $C_{6}$ would necessarily contain $\ln m^{2}$ terms in order to reproduce the quartic terms of Eq. (4.62). Thus, in case the normal-ordering prescription is applied, there is no separation of the two scales $q$ and $m$.

This example, in particular Eq. (4.63) and Fig. 4.8, shows that in principle one could still use the large-momentum procedure for the practical computation of $C_{n}(q)$ and $\left\langle\mathcal{O}_{n}(0)\right\rangle$. However, in practice it turns out that this is quite tedious.

The aim of the calculation is to obtain $R(s)$ up to order $m^{4} \alpha_{s}^{3}$, which means that due to the equation

$$
\begin{aligned}
\left.R(s)\right|_{m^{4}} & =\left.12 \pi \operatorname{Im} \Pi\left(q^{2}=s+i \epsilon\right)\right|_{m^{4}} \\
& \sim \operatorname{Im}\left[C_{1}\left\langle\mathcal{O}_{1}\right\rangle+C_{2}\left\langle\mathcal{O}_{2}\right\rangle+C_{6}\left\langle\mathcal{O}_{6}\right\rangle\right]
\end{aligned}
$$

one has to evaluate the coefficient functions and vacuum expectation values up to sufficiently high order. The imaginary parts can only arise from the coefficient functions as by construction only they can develop logarithms of the form $\ln (-s-i \epsilon)$. Note further that the information about the considered current only enters into the coefficient functions; the matrix elements of the operators are universal. For the vector current correlator it turns out that $C_{1}$ develops an imaginary part starting from $\mathcal{O}\left(\alpha_{s}^{3}\right)$. As $\left\langle\mathcal{O}_{1}\right\rangle$ is proportional to $\alpha_{s}$ there is no contribution to order $\alpha_{s}^{3}$ from this term. The lowest order of $\left\langle\mathcal{O}_{2}\right\rangle$ is $\alpha_{s}^{0}$ and the imaginary part for $C_{2}$ starts at order $\alpha_{s}^{2}$. This implies that $C_{2}$ is needed up to order $\alpha_{s}^{3}$ (three loops) and $\left\langle\mathcal{O}_{2}\right\rangle$ up to order $\alpha_{s}$ (two loops). So far the occuring integrals are all available in the literature. However, in the case of $C_{6}$ the logarithmic terms up to $\mathcal{O}\left(\alpha_{s}^{3}\right)$ 
are needed, which would require a four-loop calculation. This can be avoided as we will see in the following 73 .

We consider the renormalization group properties of the polarization function. In general $\Pi\left(q^{2}\right)$ is not renormalization group invariant. Considering, however, only the quartic mass terms we have

$$
\begin{aligned}
0 & =\left.\mu^{2} \frac{\mathrm{d}}{\mathrm{d} \mu^{2}} \Pi\left(q^{2}\right)\right|_{m^{4} \text { terms }} \\
& =\mu^{2} \frac{\mathrm{d}}{\mathrm{d} \mu^{2}}\left(C_{1}\left\langle\mathcal{O}_{1}\right\rangle+C_{2}\left\langle\mathcal{O}_{2}\right\rangle+C_{6}\left\langle\mathcal{O}_{6}\right\rangle\right),
\end{aligned}
$$

as non-zero contributions may at most have mass dimension two. Using Eq. (4.10) and

$$
\mu^{2} \frac{\mathrm{d}}{\mathrm{d} \mu^{2}} C_{6}=\left(\mu^{2} \frac{\partial}{\partial \mu^{2}}+\beta \pi \frac{\partial}{\partial \alpha_{s}}\right) C_{6}
$$

one obtains

$$
\frac{\partial}{\partial L} C_{6}\left\langle\mathcal{O}_{6}\right\rangle=-4 \gamma_{m} C_{6}\left\langle\mathcal{O}_{6}\right\rangle-\beta \pi \frac{\partial}{\partial \alpha_{s}} C_{6}\left\langle\mathcal{O}_{6}\right\rangle-C_{1} 4 m^{4} \alpha_{s} \frac{\partial}{\partial \alpha_{s}} \gamma_{0}+C_{2} 4 m^{4} \gamma_{0}
$$

with $L=\ln \left(\mu^{2} /\left(-q^{2}\right)\right)$. With the help of this equation the logarithmic terms of $C_{6}$ at order $\alpha_{s}^{3}$ can be obtained through two- and three-loop calculations. In particular $C_{6}$ itself appears on the right-hand side of Eq. (4.67), however, only at order $\alpha_{s}^{2}$ which corresponds to massless three-loop integrals.

As a simple example let us evaluate the order $\alpha_{s}^{0}$ term of Eq. (4.67). In this limit there is only a contribution from the last term. With the help of Eq. (4.11) and $C_{2}=2 / q^{4}$ we obtain

$$
\frac{\partial}{\partial L} C_{6}\left\langle\mathcal{O}_{6}\right\rangle=\frac{3}{16 \pi^{2}}\left(\frac{m^{2}}{q^{2}}\right)^{2}(-8),
$$

which after integration reproduces the logarithmic terms of the renormalized version of Eq. (4.63):

$$
C_{6}\left\langle\mathcal{O}_{6}\right\rangle=\frac{3}{16 \pi^{2}}\left(\frac{m^{2}}{q^{2}}\right)^{2}\left(-4+8 l_{q \mu}\right) .
$$

At this point it is instructive to make again a comparison with the large-momentum procedure. Applied to the polarization function there is always one term where the hard subgraph constitutes the complete diagram Taylor expanded in the masses (cf. Appendix A.1). At $n$-loop order, i.e. considering QCD corrections to order $\alpha_{s}^{n-1}$, this means that $n$-loop massless propagrator-type integrals have to be solved. Actually, as we are only interested in the imaginary part of the polarization function only the logarithmic parts 
of the integrals is needed. This part exactly constitutes the left-hand side of Eq. (4.67). The right-hand side of Eq. (4.67) contains lower-order terms of $C_{6}{ }^{\top}$ and contributions of $(n-1)$-loop diagrams. Thus the price one has to pay in order to avoid the computation of the (imaginary part) of the $n$-loop diagrams is the construction of appropriate operators, the computation of their anomalous dimension and their coefficient functions.

For the practical computation of the coefficient functions the so-called "method of projectors" [117, 56 is used. For the projectors, $\pi_{n}$, an appropriate combination of initial and final states, $|i\rangle$ and $|f\rangle$, and derivatives with respect to masses and momenta is chosen in such a way that one has

$$
\pi_{n}\left[\mathcal{O}_{m}^{0}\right]=\delta_{n m}
$$

Here $\mathcal{O}_{n}^{0}$ is one of the operators defined in Eqs. (4.1) and (4.2) and $\pi_{n}$ has the form

$$
\pi_{n}[X]=\left.\sum_{k} P_{k}\left(\frac{\partial}{\partial p}, \frac{\partial}{\partial m}\right)\left\langle f_{k}|X| i_{k}\right\rangle\right|_{p=m=0}
$$

It is understood that the nullification of $p$ and $m$ happens before the loop integrals are performed. Thus, in Eq. (4.70) there is only the tree-level contribution; all loop corrections become massless tadpoles which are set to zero in dimensional regularization. The application of the projectors to Eq. (4.61) immediately leads to

$$
C_{n}^{0}(q)=\pi_{n}\left[T^{j}(q)\right]
$$

which relates the bare coefficient function $C_{n}^{0}$ to massless two-point functions. For $C_{6}^{0}$, e.g., the projector is quite simple. It essentially consists of four derivatives of the polarization function with respect to $m$. From the structure of the operators it is clear that the projectors for $C_{1}^{0}$ and $C_{2}^{0}$ are more complicated, as the corresponding diagrams also involve external gluons and quarks, respectively [118, 74].

There is quite some similarity between the "method of projectors" and the procedure we have used for the computation of the decoupling constants in Section 3. In fact, if one tries to construct a projector for $C_{1}^{0}$ one arrives at a similar system of equations as in Eq. (4.31) [118, 74].

As already mentioned, the computation of the vacuum expectation values reduces to the evaluation of vacuum integrals which have been calculated up to three-loop order [119, 72, 120, 73, 74.

We refrain from listing the individual results for the coefficient functions and the vacuum expectation values of the operators but provide directly the results for $R(s)$. Thereby we want to list the results of those terms which contribute to the production of the heavy quark pair $Q \bar{Q}$ via the exchange of a photon which will be denoted by $R_{Q}(s)$. In this Subsection we want to list $R_{Q}(s)$ up to order $\alpha_{s}^{3} m^{4} / s^{2}$ and postpone the discussion of the remaining terms (in particular the full mass dependence at order $\alpha_{s}^{2}$ ) to Section 5.2.1.

\footnotetext{
${ }^{17}$ Note that $\gamma_{m}$ and $\beta$ start at $\mathcal{O}\left(\alpha_{s}\right)$ and $\mathcal{O}\left(\alpha_{s}^{2}\right)$, respectively.
} 
It is convenient to decompose the contributions to $R_{Q}(s)$ into three parts

$$
R_{Q}(s)=3\left(Q_{Q}^{2} r_{Q}+\sum_{q} Q_{q}^{2} r_{q Q}+r_{Q, \text { sing }}\right)
$$

where the sum runs over all massless quark flavours $q$ and $Q_{q}$ denotes the charge of quark $q$. In Eq. (4.73) we distinguish the contributions where the massive quark $Q$ directly couples to photon $\left(r_{Q}\right)$ from the ones where in a first step a massless quark is produced which subsequently splits into the massive quark $Q$. Furthermore, the singlet contributions are displayed separately. They arise from diagrams where the external current couples to a closed quark line which is different from the one involving the final-state quarks. In the

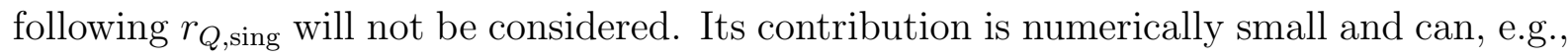
be found in [121.

Both $r_{Q}$ and $r_{q Q}$ are expanded in $m_{q}^{2} / s$ and can be written as

$$
\begin{aligned}
r_{Q} & =r_{0}+r_{Q, 2}+r_{Q, 4}+\ldots, \\
r_{q Q} & =r_{0}+r_{q Q, 2}+r_{q Q, 4}+\ldots,
\end{aligned}
$$

where $r_{0}$ belongs to the massless approximation, while $r_{Q, n}$ and $r_{q Q, n}$ contain the mass terms of order $m_{Q}^{n}$. A look to the contributing diagrams shows that the contributions to $r_{q Q, 2}$ and $r_{q Q, 4}$ arise for the first time at order $\alpha_{s}^{2}$. However, it can be inferred from general renormalization group considerations that the corresponding coefficient of $r_{q Q, 2}$ has to be zero [113, 122], which means that it starts out only at order $\alpha_{s}^{3}$.

The numerical result for the massless approximation reads 60, 39, 38.

$$
\begin{aligned}
r_{0}=1 & +\frac{\alpha_{s}}{\pi}+\left(\frac{\alpha_{s}}{\pi}\right)^{2}\left(1.98571-0.115295 n_{f}\right) \\
& +\left(\frac{\alpha_{s}}{\pi}\right)^{3}\left(-6.63694-1.20013 n_{f}-0.00517836 n_{f}^{2}\right)+\ldots
\end{aligned}
$$

where the ellipses indicate higher orders in $\alpha_{s} . n_{f}$ is the number of active quark flavours. The quadratic mass corrections are given by 114, 113

$$
\begin{aligned}
r_{Q, 2}= & \frac{m_{Q}^{2}}{s} \frac{\alpha_{s}}{\pi}\left[12+\frac{\alpha_{s}}{\pi}\left(126.5-4.33333 n_{f}\right)\right. \\
& \left.+\left(\frac{\alpha_{s}}{\pi}\right)^{2}\left(1032.14-104.167 n_{f}+1.21819 n_{f}^{2}\right)\right], \\
r_{q Q, 2}= & \frac{m_{Q}^{2}}{s}\left(\frac{\alpha_{s}}{\pi}\right)^{3}\left[-7.87659+0.35007 n_{f}\right]+\ldots,
\end{aligned}
$$

and, finally, for the quartic terms we have [73, 74, [21]

$$
r_{Q, 4}=\left(\frac{m_{Q}^{2}}{s}\right)^{2}\left[-6-22 \frac{\alpha_{s}}{\pi}\right.
$$




$$
\begin{aligned}
&+\left(\frac{\alpha_{s}}{\pi}\right)^{2}\left(148.218-6.5 l_{m s}+\left(-1.84078+0.333333 l_{m s}\right) n_{f}\right) \\
&+\left(\frac{\alpha_{s}}{\pi}\right)^{3}\left(4800.95-244.612 l_{m s}+13 l_{m s}^{2}\right. \\
&+\left(-275.898+18.1861 l_{m s}-0.666667 l_{m s}^{2}\right) n_{f} \\
&\left.\left.+\left(4.97396-0.185185 l_{m s}\right) n_{f}^{2}\right)+\ldots\right] \\
& r_{q Q, 4}=\left(\frac{m_{Q}^{2}}{s}\right)^{2}\left(\frac{\alpha_{s}}{\pi}\right)^{2}\left[-0.474894-l_{m s}\right. \\
& \quad+\frac{\alpha_{s}}{\pi}\left(4.59784-22.8619 l_{m s}+2 l_{m s}^{2}\right. \\
&\left.\left.\quad+\left(0.196497+0.88052 l_{m s}\right) n_{f}\right)+\ldots\right]
\end{aligned}
$$

with $l_{m s}=\ln m_{Q}^{2} / s$.

In Eqs. (4.76) and (4.77) the $\overline{\mathrm{MS}}$ quark mass has been chosen as a parameter. This is inherent to the method used for the computation. Actually also the mass which is present in the renormalized operators $\mathcal{O}_{2}$ and $\mathcal{O}_{6}$ is defined in the modified minimal subtraction scheme. In order to transform the expressions into the on-shell scheme the two- [123] and three-loop [124, 125, 126] (see also Section 5.4) relation between the masses is necessary ${ }^{18}$. We obtain for the quadratic terms (see also Ref. [127])

$$
\begin{aligned}
r_{Q, 2}^{\mathrm{OS}}= & \frac{M_{Q}^{2}}{s} \frac{\alpha_{s}}{\pi}\left[12+\frac{\alpha_{s}}{\pi}\left(94.5000+24 L_{m s}-4.33333 n_{f}\right)+\left(\frac{\alpha_{s}}{\pi}\right)^{2}\left(347.168+378 L_{m s}\right.\right. \\
& \left.\left.-9 L_{m s}^{2}+n_{f}\left(-67.6190-17.3333 L_{m s}+2 L_{m s}^{2}\right)+1.21819 n_{f}^{2}\right)+\ldots\right], \\
r_{q Q, 2}^{\mathrm{OS}}= & \frac{M_{Q}^{2}}{s}\left(\frac{\alpha_{s}}{\pi}\right)^{3}\left[-7.87659+0.35007 n_{f}\right]+\ldots,
\end{aligned}
$$

where $L_{m s}=\ln \left(M_{Q}^{2} / s\right)$. Note the presence of mass logarithms which are introduced via the transition to the on-shell scheme.

The quartic terms read in the on-shell scheme

$$
\begin{aligned}
r_{Q, 4}^{\mathrm{OS}}= & \left(\frac{M_{Q}^{2}}{s}\right)^{2}\left[-6+\frac{\alpha_{s}}{\pi}\left(10-24 L_{m s}\right)+\left(\frac{\alpha_{s}}{\pi}\right)^{2}\left(570.519-155.5 L_{m s}-15 L_{m s}^{2}\right.\right. \\
& \left.+n_{f}\left(-26.8336+9 L_{m s}-2 L_{m s}^{2}\right)\right)+\left(\frac{\alpha_{s}}{\pi}\right)^{3}\left(9157.82-444.899 L_{m s}\right. \\
& -147.750 L_{m s}^{2}+7.50000 L_{m s}^{3}+n_{f}\left(-936.140+243.009 L_{m s}-26.1667 L_{m s}^{2}\right. \\
& \left.-0.666667 L_{m s}^{3}\right)+n_{f}^{2}\left(20.6385-7.86797 L_{m s}+1.44444 L_{m s}^{2}\right. \\
& \left.\left.\left.-0.222222 L_{m s}^{3}\right)\right) \ldots\right],
\end{aligned}
$$

\footnotetext{
${ }^{18}$ Due to the absence of a Born term in Eq. (4.76) the two-loop relation between the $\overline{\mathrm{MS}}$ and on-shell quark mass is sufficient in this case.
} 


$$
\begin{aligned}
r_{q Q, 4}^{\mathrm{OS}}= & \left(\frac{M_{Q}^{2}}{s}\right)^{2}\left(\frac{\alpha_{s}}{\pi}\right)^{2}\left[-0.474894-L_{m s}+\frac{\alpha_{s}}{\pi}\left(9.79728-21.4282 L_{m s}-2 L_{m s}^{2}\right.\right. \\
& \left.\left.+n_{f}\left(0.196497+0.880520 L_{m s}\right)\right) \ldots\right] .
\end{aligned}
$$

Note that in this case the transition to the on-shell scheme even introduces cubic masslogarithms.

In Fig. $4.9 r_{c, 4}, r_{b, 4}$ and $r_{t, 4}$ are shown as a function of the center-of-mass energy, $\sqrt{s}$, where successively higher orders in $\alpha_{s}$ are taken into account. It can be seen that the major part of the result is given by the Born approximation. The correction terms of order $\alpha_{s}, \alpha_{s}^{2}$ and $\alpha_{s}^{3}$ are significantly smaller than the leading terms. However, it can be observed that with increasing order they remain roughly comparable in magnitude which could indicate a bad behaviour of the perturbative expansion. Nevertheless, the higher orders are small compared to the $m_{Q}^{4} / s^{2}$ Born terms. It was shown in [121] that the overall prediction for $R(s)$ is stable and a variation of $\mu$ between $\sqrt{s} / 2$ and $2 \sqrt{s}$ for $r_{c, 4}$ at $6 \mathrm{GeV}$ varies by \pm 0.0005 and for $r_{b, 4}$ at $14 \mathrm{GeV}$ by \pm 0.0016 . In the case of $r_{t, 4}$ the variation is negligible.

Thus a prediction for $R_{Q}(s)$ up to order $\alpha_{s}^{3}$ is available. It includes mass terms in an expansion up to the quartic order.

\section{Asymptotic expansion and Padé approximation}

Using the techniques currently available, diagrams beyond two loops can only be computed for special cases. In particular, only diagrams depending on one scale, like an external momentum or an internal mass, have been studied systematically. This section is devoted to a method which allows for the numerical reconstruction of a function depending on two dimensionful parameters like an external momentum $q$ and a mass $M$. It constitutes a powerful combination of asymptotic expansion and the analytic structure of the function to be approximated.

The basic idea is as follows. Let us consider a Feynman diagram depending on an external momentum $q$ and one mass parameter, $M$, which can occur in some of the internal lines. Then the final result is a function of $M^{2} / q^{2}$, which in general is quite involved and at three-loop order - at least with the current techniques - not computable in an analytical form. On the other hand, it is straightforward [TI to evaluate the diagram in the limits $q^{2} \ll M^{2}$ and $q^{2} \gg M^{2}$. The information from the different kinematical regions is combined and a semi-numerical function of $M^{2} / q^{2}$ is constructed. Below we will demonstrate on typical examples that it provides a very good approximation to the exact result.

In Subsection 5.1 we start with a detailed description of the method and present explicit results for a two-loop example. The physical processes discussed afterwards in

\footnotetext{
${ }^{19}$ With "straightforward" we mean that program packages exist which allow the computation of the corresponding expressions with the help of computers. In this context see also the Appendices A.2 and A.3.
} 

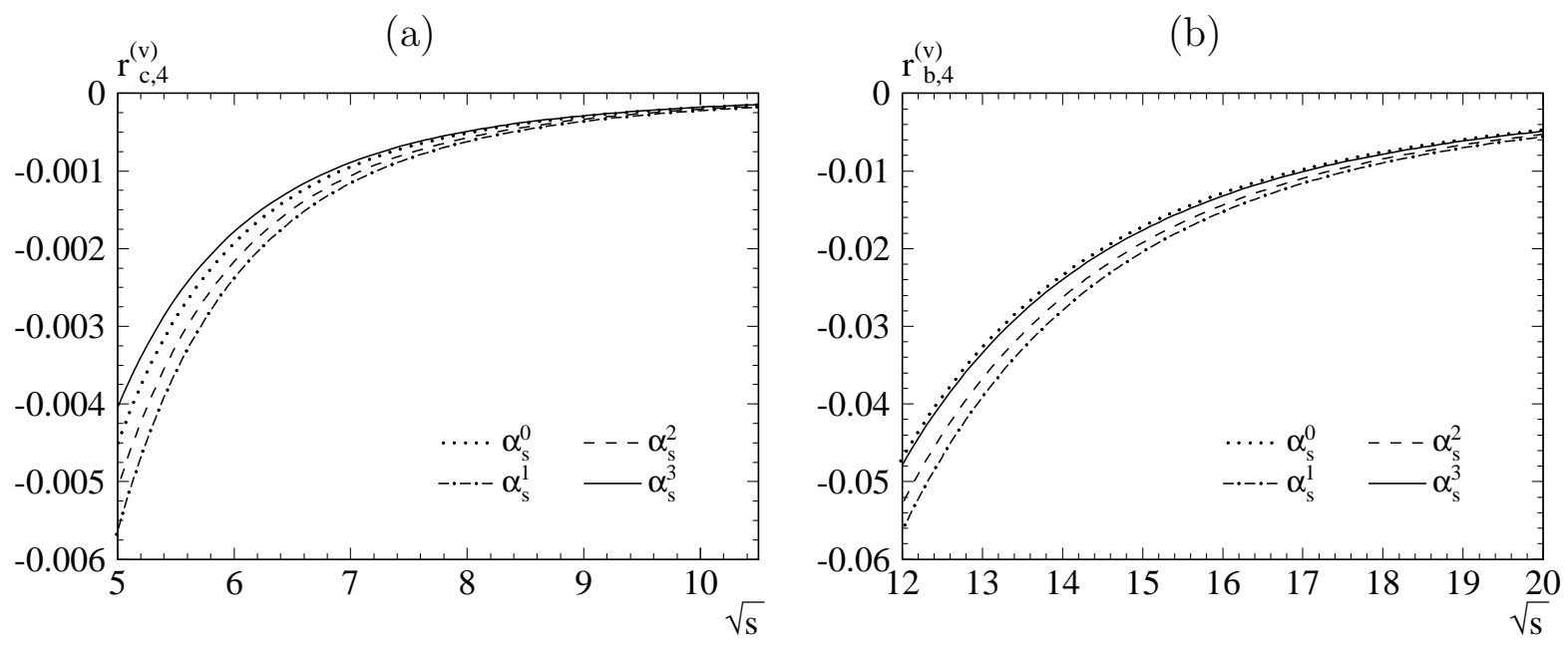

(c)

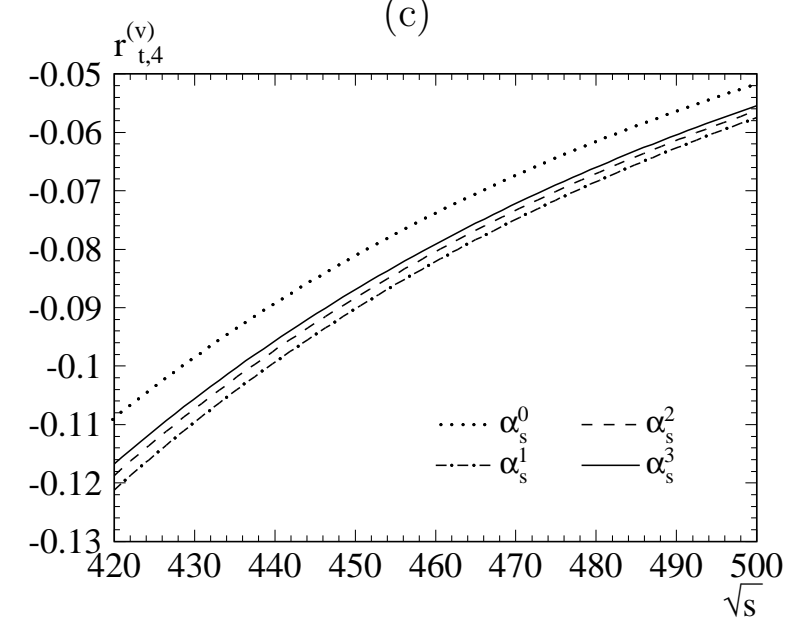

Figure 4.9: Quartic mass corrections $\left(\propto m^{4}\right)$ to the non-singlet contribution of $r_{c}$ (a), $r_{b}$ (b), and $r_{t}$ (c) arising from diagrams where the external current couples directly to the massive quark.

Sections 5.2, 5.3 and 5.4 point out different aspects and fields of application. In the first application we consider current correlators at three loops both for a diagonal and a non-diagonal coupling to quarks. In the latter case we assume that one of the quarks is massless. The correlators depend on the external momentum $q$ and the quark mass $M$. The main interest is in the imaginary part which represents a physical observable.

Also the two-point function considered in Subsection 5.4 - proper combinations of the quark selfenergy - depends on an external momentum and one mass parameter. However, the main interest is not on the functional behaviour but on the value at threshold, i.e. $q^{2}=M^{2}$. The successful application of our method in that case is not obvious, especially as only the information for $q^{2} \ll M^{2}$ and $M^{2} \ll q^{2}$ are incorporated.

In Subsection 5.3 four-loop integrals are computed in order to obtain the order $\alpha^{2}$ QED 
corrections to the muon decay. As we are only interested in the imaginary part they can be reduced to three-loop integrals using asymptotic expansions. From the technical point of view we want to obtain the value of a function for $q^{2}=M^{2}$ using only expansion terms for $q^{2} \ll M^{2}$. Also here the Padé approximation turns out to be quite successful. We want to mention already here that the example of the muon decay does not fit completely into the philosophy which is developed in Section 5.1 as no high-energy information can be incorporated. This leads to worse approximations. Furthermore, in a first step the imaginary part is taken and afterwards the Padé approximation is applied.

\subsection{The Method}

The basic ingredients and tools of our method are moments of the function to be considered, conformal mapping and Padé approximation. In this Section we will explain the role of each of them.

The aim is to obtain an approximation to the function $f(z)$ which can not be computed directly. The analytical properties of $f(z)$ are exploited and expansions of $f(z)$ for small and large argument are used in combination with a conformal mapping and Padé approximation. In what follows we have in mind the computation of approximations to a physical function, like a vector boson self energy, at a given loop-order 0 . In particular, we assume that $f(z)$ is analytical for $z \rightarrow 0$. However, in the limit $1 / z \rightarrow 0$ we allow for a non-analytical behaviour.

In general a Padé approximation of a function $f(z)$ is defined through

$$
[n / m](z)=\frac{a_{0}+a_{1} z+\ldots a_{n} z^{n}}{1+b_{1} z+\ldots b_{m} z^{m}}
$$

where the coefficients $a_{i}$ and $b_{j}$ are determined from the requirement that the Taylor expansion of Eq. (5.1) coincides with the first $n+m+1$ terms of the Taylor expansion of $f(z)$ around $z=0$. Thus, in case the Taylor expansion is known up to terms of order $z^{k}$ Padé approximations $[n / m]$ fulfilling the condition

$$
k-1 \geq n+m
$$

can be computed.

In the approach discussed in the remaining part of this Section the considerations of the previous paragraph are improved with respect to two points. First, we perform the Padé approximation not only in $z$ but also in a new variable which is confined to the interior of the unit circle and thus provides better convergence properties. Second, we want to include in the approximation for $f(z)$ not only Taylor coefficients around $z=0$ but also information from other kinematical regions, in particular from $z \rightarrow \infty$.

\footnotetext{
${ }^{20}$ This is in contrast to the considerations of Ref. [128] where the Padé approximation has been performed in the coupling constant in order to estimate higher order terms in $\alpha_{s}$.

${ }^{21}$ In general also Taylor expansion around $z_{0} \neq 0$ can be considered. However, for our purpose the choice $z_{0}=0$ is sufficient.
} 
Our procedure is restricted to the approximation of functions which depend on one dimensionless variable, $z$. Concerning the physical applications we have in mind twopoint functions where $z$ is given by the ratio of the squared external momentum and the square of an internal mass. This motivates the following form of $f(z)$ for small and large argument 22

$$
f(z)= \begin{cases}\sum_{k=1}^{n_{\text {sma }}} c_{k} z^{k} & \text { for } z \rightarrow 0 \\ \sum_{k=0}^{n_{\operatorname{lar}}} \sum_{i \geq 0} d_{k, i}[\ln (-z)]^{i} z^{-k} & \text { for } z \rightarrow-\infty\end{cases}
$$

where the normalization $f(0)=0$ has been chosen. As we will see below, in the computation of the coefficients $c_{k}$ and $d_{k, i}$ - in the following also refered to as moments - one of the scales drops out and the integrals to be evaluated are much simpler. The moments $c_{k}$ and $d_{k, i}$ will serve as input for our procedure. In addition we also admit information about the behaviour at the physical threshold, which we choose to be at $z=1$, as input.

In order to obtain a semi-numerical approximation of the function $f(z)$ the following steps have to be performed:

1. Compute as many moments as possible for small and large $z$. As we require analyticity for $z \rightarrow 0$ one gets in this limit a simple Taylor series of the Feynman diagrams in the external momentum. The expansion in $q$ can be performed before the momentum integrations are performed. As a result the external momentum no longer appears in the integrand and one ends up with vacuum diagrams. They are analytically known up to three-loop order in case of one internal mass parameter (cf. Appendix A.2).

However, for $z \rightarrow-\infty$ the rules of asymptotic expansion [129 have to be applied. As a consequence the number of individual terms to be considered in the practical computation is larger. However, also here the number of scales in the individual diagrams is reduced. One ends up with either vacuum integrals or massless twopoint functions. The latter are responsible for the logarithmic terms in $z$.

2. Incorporate the information for $z \rightarrow 1$ which we denote as $f^{\text {thr }}(z)$. In the physical examples considered below this information is either logarithmical, i.e. of the form $\ln (1-z)$, or proportional to $1 / \sqrt{1-z}$. The latter occurs, for instance, in the abelian contribution to the vector current correlator (cf. Section 5.2) and corresponds to the Coulomb singularity. The further steps slightly depend on which case is present.

In case the leading threshold behaviour is logarithmic one constructs $f^{\text {thr }}(z)$ in such a way that the singularity is reproduced for $z \rightarrow 1$. One has to take care that $f^{\text {thr }}(z)$ does not destroy the behaviour of $f(z)$ for small and large $z$. In particular, the expansion of $f^{t h r}(z)$ has to be analytical for $z \rightarrow 0$. By construction the difference $f(z)-f^{t h r}(z)$ is regular for $z \rightarrow 1$ and has the same limiting behaviour as the one

\footnotetext{
${ }^{22}$ It is advantageous to consider the space-like region of $z$ where no imaginary part occurs.
} 
required in Eq. (5.3). We should mention already here that due to the construction of the Padé method the resulting function has a vanishing imaginary part at $z=1$. Thus it is crucial to implement the leading threshold behaviour in this way.

Threshold singularities of the form $1 / \sqrt{1-z}$ are not taken into account at this step, i.e. formally $f^{\text {thr }}(z)=0$ is chosen. They are treated below. In contrast to the logarithmic singularities they are removed via multiplication and not by subtractions.

3. Construct a function $f^{\log (z)}$ in such a way that the combination

$$
\tilde{f}(z) \equiv f(z)-f^{t h r}(z)-f^{\log }(z),
$$

is polynomial both in $z$ and $1 / z$, i.e. in the small- and high-energy region. Furthermore no logarithmic singularities may be introduced for $z \rightarrow 1$.

In this step a large part of information (e.g., the large high-energy logarithms) which is known analytically is extracted and only a small remainder $\tilde{f}(z)$ is left. It parameterizes the unknown part of $f(z)$.

4. Perform a conformal mapping. The change of variables [130]

$$
z=\frac{4 \omega}{(1+\omega)^{2}},
$$

maps the $z$ plane into the interior of the unit circle of the $\omega$ plane. Thereby the cut $[1, \infty)$ is mapped to the perimeter. The conformal mapping is visualized in Fig. 5.1.

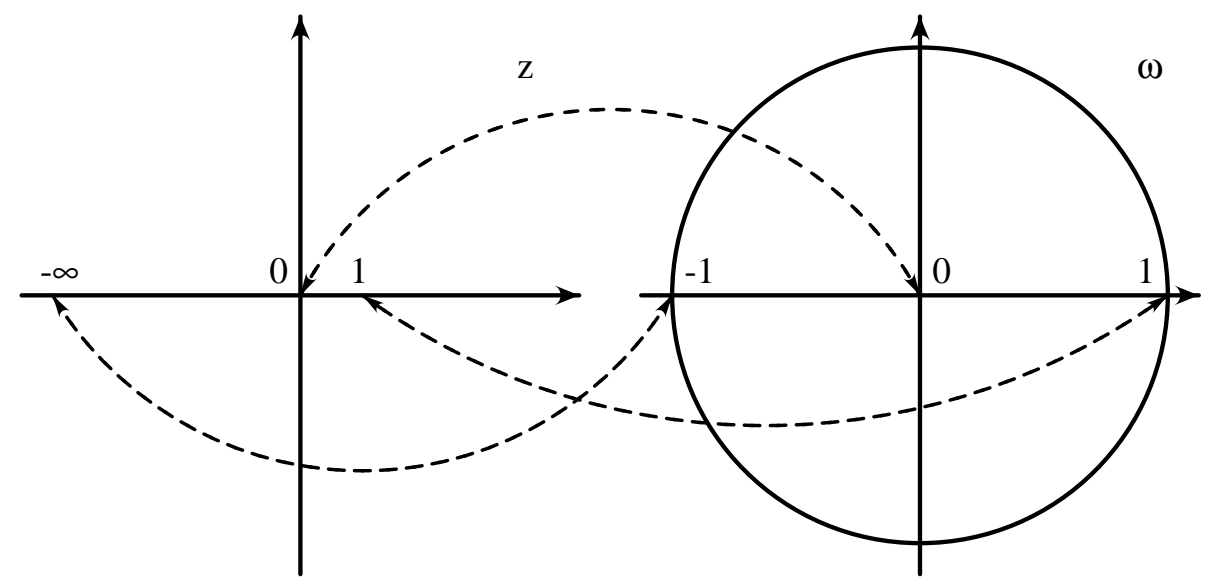

Figure 5.1: The conformal mapping (5.5) maps the $z$ plane into the interior of the unit circle in the $\omega$ plane.

5. In a next step a function is defined for which finally the Padé approximation is performed. Due to the discussion in the context of Eq. (5.1) we are interested to 
shift the information available for $\omega \rightarrow-1$ to $\omega \rightarrow 0$. Furthermore we have to take care of possible power-like threshold singularities.

Following Ref. [131] we define

$$
P_{n_{\text {lar }}}(\omega)=\frac{p^{t h r}(\omega)(4 \omega)^{n_{\text {lar }}-1}}{(1+\omega)^{2 n_{\text {lar }}}}\left(\tilde{f}(z)-\sum_{j=0}^{n_{\text {lar }}-1} \frac{1}{j !}\left(\left.\frac{d^{j}}{d(1 / z)^{j}} \tilde{f}(z)\right|_{z=-\infty}\right) \frac{(1+\omega)^{2 j}}{(4 \omega)^{j}}\right)
$$

where for $\tilde{f}$ the moments up to order $1 / z^{n_{\text {lar }}}$ must be known. The function $p^{\text {thr }}(\omega)$ is equal to 1 in case $f(z)$ has logarithmic divergences at threshold and $p^{\text {thr }}(\omega)=(1-\omega)$ if $f(z)$ is proportional to $1 / \sqrt{1-z}$ for $z \rightarrow 1$. The available information from the moments transforms into $P_{n_{\text {lar }}}(-1)$ and $P_{n_{\text {lar }}}^{(k)}(0),\left(k=0,1, \ldots, n_{\text {lar }}+n_{\text {sma }}-1\right)$, where $n_{\text {sma }}$ is the number of moments for $z \rightarrow 0$. Whereas for a logarithmic threshold behaviour the corresponding information is already taken into account in step 2 , the $1 / \sqrt{1-z}$ behaviour is treated with the factor $p^{t h r}(\omega)$ and in addition $P_{n_{\text {lar }}}(\omega)$ is known for $\omega=1$.

6. In the last step a Padé approximation is performed for the function $P_{n_{\text {lar }}}(\omega)$. This means that $P_{n_{\text {lar }}}(\omega)$ is identified with a function $[n / m](\omega)$ as defined in Eq. (5.1), where the number of coefficients on the right-hand side depends on the amount of information available for $P_{n_{l a r}}(\omega)$. In particular, one has $n+m=n_{\text {lar }}+n_{\text {sma }}+1$ if $P_{n_{\text {lar }}}(1)$ is available and otherwise $n+m=n_{\text {lar }}+n_{\text {sma }}$. This leads to a system of (non-linear) equations which can be solved for the coefficients $a_{i}$ and $b_{j}$ in Eq. (5.1). For large values of $n+m$ the analytical solution becomes quite lengthy and time consuming. Thus it is preferable to solve the equations numerically (using high precision).

7. Finally, Eq. (5.6) has to be solved for $\tilde{f}(z)$ and from Eq. (5.4) an approximation for the function $f(z)$ is obtained.

Due to the structure of Eq. (5.1) some Padé approximants develop poles inside the unit circle $(|\omega| \leq 1)$. In general we will discard such results as they would induce unphysical poles in the $z$-plane. In some cases, however, a pole coincides with a zero of the numerator up to several digits accuracy. These Padé approximations will be taken into account in constructing our results. If not stated otherwise we will, in addition to the Padé results without any poles inside the unit circle, also use the ones where the poles are accompanied by zeros within a circle of radius 0.01 , and the distance between the pole and the physically relevant point $q^{2} / M^{2}=1$ is larger than 0.1 .

There are situations where the information for $z \rightarrow-\infty$ can not be used as this would lead to physically not allowed scenarios (cf. Subsection 5.3). In this case it is not necessary (and even not possible) to define the function $P_{n_{l a r}}(\omega)$. Instead one can directly perform a Padé approximation either in $z$ or in $\omega$.

At this point we should spend some words on the estimation of the errors to be assigned to the final results. It is difficult to provide general rules for their determination as it very 
much depends on the problem under consideration. Experience on the estimation of the error can be gained from the comparison with known results at lower order or for other colour structures. A reasonable choice for the systematic error due to the used method is to take the spread of the individual Padé results.

Concerning the above list some comments to point 3 are in order. In principle there are many ways to subtract the high-energy logarithms. However, one has to keep in mind that the subtraction must not spoil the polynomial behaviour for small $z$. Furthermore, no divergences may be introduced. In particular, $f^{\log }(z)$ has to be regular for $z=1$.

For the construction of $f^{\log }(z)$ it is convenient to use the function

$$
G(z)=\frac{2 u \ln u}{u^{2}-1}
$$

with

$$
u=\frac{\sqrt{1-\frac{1}{z}}-1}{\sqrt{1-\frac{1}{z}}+1}
$$

which naturally occurs in the result of the one-loop photon polarization function, as a building block. That this is possible in a systematic way can best be seen by looking at the expansion of $G(z)$ in the different kinematical regions

$$
G(z)= \begin{cases}1+\frac{2}{3} z+\frac{8}{15} z^{2}+\mathcal{O}\left(z^{3}\right) & z \rightarrow 0 \\ \frac{1}{2 z} \ln (-1 / 4 z)+\frac{1}{4 z^{2}}(1+\ln (-1 / 4 z))+\mathcal{O}\left(\frac{1}{z^{3}}\right) & z \rightarrow-\infty \\ \frac{\pi}{2 \sqrt{1-z}}-1+\mathcal{O}(\sqrt{1-z}) & z \rightarrow 1\end{cases}
$$

From this equation one can see that $f^{\log }(z)$ can be chosen as a linear combination of terms $(1 / z)^{j}(1-z)^{l} z^{m}(G(z))^{n}(j, l, m, n \geq 0)$ where the corresponding coefficients are determined as follows:

- Consider the term in the second equality of Eq. (5.3) which has the lowest value of $k$ and the highest value of $i \neq 0$ and fix the index $n$ such that the powers of the logarithms coincide.

- Determine $l$ in such a way that there is no singular behaviour for $z \rightarrow 1$.

- As $G(z)$ starts with order $1 / z$ for $z \rightarrow-\infty$ one eventually has to correct for it with the help of the index $m$.

- In a similar way the index $j$ is used in order to subtract the logarithms suppressed by higher powers in $1 / z$.

- Finally, terms involving $l=m=n=0$ and $j \geq 0$ are added in order to restore the behaviour for $z \rightarrow 0$. 
- Repeat the procedure with the next values of $k$ and $i$, i.e. lower $i$ by one unit until $i=0$ is reached; then increase $k$ by one unit.

By construction this algorithm terminates once the linear logarithm of the largest highenergy moment is treated.

To our knowledge a simple version of the method was first applied in [132] for the evaluation of certain four-loop contributions to the anomalous magnetic moment of the muon. It was obtained by a convolution over the photon polarization function. For the latter an approximation formulae was obtained with the help of the Padé-method. One should stress that for this application only the integral over the space-like momenta of the approximation was used. In the applications which are discussed in this review the approximated function itself and in particular its analyticity properties are of interest. A brief introduction to the Padé-method and the discussion of some results can also be found in [133].

\subsubsection{Explicit example at two loops}

For clarity let us present an example where in all steps explicit results are given. We consider two-loop QCD corrections to the correlator of two vector currents (for a precise definition see Eq. (5.15) below).

1. The expansion of the diagrams for small external momentum leads to two-loop vacuum integrals which, e.g., can be computed with the help of MATAD 35. After renormalizing the quark mass in the on-shell scheme and subtracting the constant, the result for the first three expansion terms reads

$$
\Pi^{(1), v}\left(q^{2}\right)=\frac{3}{16 \pi^{2}}\left(\frac{328}{81} z+\frac{1796}{675} z^{2}+\frac{999664}{496125} z^{3}+\ldots\right) .
$$

In the high-energy region we restrict ourselves to the first two terms which can be obtained by solving massless integrals with the help of MINCER [31]. Using again the on-shell quark mass definition and taking into account the condition $\Pi(0)=0$ gives

$$
\Pi^{(1), v}(z)=\frac{3}{16 \pi^{2}}\left(\frac{5}{6}-4 \zeta_{3}-\ln (-4 z)-\frac{3}{z} \ln (-4 z)+\ldots\right) .
$$

2. At threshold $\Pi(z)$ has a logarithmic singularity which can be cast in the form

$$
\Pi^{(1), v, t h r}(z)=\frac{3}{16} \ln \left(\frac{1}{1-z}\right) .
$$

Thus, the combination $\Pi(z)-\Pi^{t h r}(z)$ is constant for $z=1$, has a polynomial behaviour for $z \rightarrow 0$ and at most logarithmic singularities for $z \rightarrow-\infty$. 
3. The high-energy logarithms are subtracted with the help of the function

$$
\begin{aligned}
\Pi^{(1), v, \log }(z)= & \frac{3}{16 \pi^{2}} \frac{1}{3 z}\left[-21+z+\pi^{2}(3+5 z)\right. \\
& \left.+3(-1+z)\left(-7-2 z+\pi^{2}(1+2 z)\right) G(z)\right]
\end{aligned}
$$

which is constructed using the algorithm outlined above. The resulting function $\tilde{\Pi}^{(1), v}(z)=\Pi^{(1), v}(z)-\Pi^{(1), v, t h r}(z)-\Pi^{(1), v, l o g}(z)$ is analytical for $z \rightarrow 0$ and free of logarithms in the first two high-energy terms.

After the conformal mapping (cf. Eq. (5.5)) and the [2/2] Padé approximation in $\omega$ are performed one obtains for $\tilde{\Pi}^{(1), v}(z)$

$$
\tilde{\Pi}^{(1), v}(z)=-0.874397+\frac{\left(0.874397+0.905702 \omega+0.165184 \omega^{2}\right)(1+\omega)^{2}}{1+0.860764 \omega+0.069525 \omega^{2}}
$$

which finally leads to $\Pi_{\text {appr }}^{(1), v}(z)=\tilde{\Pi}^{(1), v}(z)+\Pi^{(1), v, t h r}(z)+\Pi^{(1), v, l o g}(z)$. In Eq. (5.14) the numbers are truncated. Usually high numerical precision is needed in order not to loose significant digits in the final result.

By construction, $\Pi_{a p p r}^{(1), v}(z)$ has the same analyticity properties as the exact function. As in this case the latter is known one can also check the quality of the approximation. It turns out that even for the relatively small amount of input used here there is a perfect agreement between $\Pi_{a p p r}^{(1), v}(z)$ and $\Pi^{(1), v}(z)$. E.g., it is not possible to distinguish the imaginary parts plotted in the range $0<2 \mathrm{~m} / \sqrt{s}<1$ [134, 135].

\subsection{Current correlators in QCD}

A variety of important observables can be described by the correlators of two currents. If the coupling of the currents to quarks is diagonal quantities like $e^{+} e^{-}$annihilation into hadrons and the decay of the $Z$ boson are covered by the vector and axial-vector current correlators. Total decay rates of CP even or CP odd Higgs bosons can be obtained by the scalar and pseudo-scalar current densities, respectively. For these cases the full mass dependence at order $\alpha_{s}^{2}$ has been computed in [134 for the non-singlet and in 131 for the singlet correlators. In Subsection 5.2.1 these results will be briefly reviewed.

On the other hand the correlators involving different quarks describe, e.g., properties of a charged gauge or Higgs boson. In particular a certain (gauge invariant) class of corrections to the single-top-quark production via the process $q \bar{q} \rightarrow t \bar{b}$ becomes available. As an application of the (pseudo-)scalar current correlator we want to mention the decay of a charged Higgs boson, which occurs in extensions of the SM, into a massive and a massless quark. Another important application of the non-diagonal current correlator is connected to the meson decay constant. Within the heavy quark effective QCD it is related to the spectral density, evaluated near threshold. The latter can be obtained from the correlator of the full theory which is considered below. 
Let us in a first step introduce the polarization functions for the four cases of interest. The vector and axial-vector $(\delta=v, a)$ correlators are defined as

$$
\left(-q^{2} g_{\mu \nu}+q_{\mu} q_{\nu}\right) \Pi^{\delta}\left(q^{2}\right)+q_{\mu} q_{\nu} \Pi_{L}^{\delta}\left(q^{2}\right)=i \int \mathrm{d} x \mathrm{e}^{i q x}\left\langle 0\left|T j_{\mu}^{\delta}(x) j_{\nu}^{\delta \dagger}(0)\right| 0\right\rangle,
$$

and the scalar and pseudo-scalar ones $(\delta=s, p)$ read

$$
q^{2} \Pi^{\delta}\left(q^{2}\right)=i \int \mathrm{d} x \mathrm{e}^{i q x}\left\langle 0\left|T j^{\delta}(x) j^{\delta \dagger}(0)\right| 0\right\rangle
$$

The currents are given by

$$
j_{\mu}^{v}=\bar{\psi}_{1} \gamma_{\mu} \psi_{2}, \quad j_{\mu}^{a}=\bar{\psi}_{1} \gamma_{\mu} \gamma_{5} \psi_{2}, \quad j^{s}=\frac{m(\mu)}{M} \bar{\psi}_{1} \psi_{2}, \quad j^{p}=i \frac{m(\mu)}{M} \bar{\psi}_{1} \gamma_{5} \psi_{2}
$$

Here $m$ is the $\overline{\mathrm{MS}}$ and $M$ the on-shell quark mass. In Eqs. (5.15) and (5.16) two powers of $q$ are factored out in order to end up with dimensionless quantities $\Pi^{\delta}\left(q^{2}\right)$. As we are mainly interested in the imaginary part, the overall renormalization can be performed in such a way that this is possible. Furthermore it is advantageous to adopt the QED-like renormalization $\Pi^{\delta}(0)=0$.

The physical observable $R(s)$ is related to $\Pi\left(q^{2}\right)$ by

$$
\begin{array}{ll}
R^{\delta}(s)=12 \pi \operatorname{Im} \Pi^{\delta}\left(q^{2}=s+i \epsilon\right) & \text { for } \delta=v, a, \\
R^{\delta}(s)=8 \pi \operatorname{Im} \Pi^{\delta}\left(q^{2}=s+i \epsilon\right) & \text { for } \delta=s, p .
\end{array}
$$

It is convenient to define

$$
\begin{aligned}
\Pi^{\delta}\left(q^{2}\right) & =\Pi^{(0), \delta}\left(q^{2}\right)+\frac{\alpha_{s}\left(\mu^{2}\right)}{\pi} C_{F} \Pi^{(1), \delta}\left(q^{2}\right)+\left(\frac{\alpha_{s}\left(\mu^{2}\right)}{\pi}\right)^{2} \Pi^{(2), \delta}\left(q^{2}\right)+\ldots \\
\Pi^{(2), \delta} & =C_{F}^{2} \Pi_{A}^{(2), \delta}+C_{A} C_{F} \Pi_{N A}^{(2), \delta}+C_{F} T n_{l} \Pi_{l}^{(2), \delta}+C_{F} T \Pi_{F}^{(2), \delta}+C_{F} T \Pi_{S}^{(2), \delta}
\end{aligned}
$$

and similarly for $R^{\delta}(s)$. The abelian contribution $\Pi_{A}^{(2), \delta}$ is already present in (quenched) QED and $\Pi_{N A}^{(2), \delta}$ originates from the non-abelian structure specific for QCD. The polarization functions containing a second massless or massive quark loop are denoted by $\Pi_{l}^{(2), \delta}$ and $\Pi_{F}^{(2), \delta}$, respectively. $\Pi_{S}^{(2), \delta}$ represents the double-triangle contribution.

Actually, we are mainly interested in the imaginary part $R^{\delta}(s)$ which in principle could be obtained from tree diagrams with five external legs, from one-loop four-point integrals and from two-loop three-point integrals. However, in particular the latter can not be evaluated analytically using current methods. Also numerically the treatment of these integrals is inconvenient. On the other hand, if one has to rely on approximations like small or large external momenta it is much more advantageous to stick to two-point functions simply because the resulting integrals are easier to solve and the corresponding techniques are much more advanced. 


\subsubsection{Diagonal current correlators}

The diagonal correlators with all their applications have extensively been discussed in the literature. Therefore we will be brief in this subsection and mainly refer to the original literature where in most cases also the analytical results can be found.

It is useful to define dimensionless variables

$$
z=\frac{q^{2}}{4 m^{2}}, \quad x=\frac{2 m}{\sqrt{s}},
$$

where $q$ is the external momentum of the polarization function and $s$ corresponds to the center-of-mass energy in the process $e^{+} e^{-} \rightarrow$ hadrons or the mass of the boson in case decay processes are considered. Then the velocity, $v$, of one of the produced quarks reads

$$
v=\sqrt{1-x^{2}}
$$

Every time the generic index $\delta$ appears without further explanation it is understood that $\delta$ represents one of the letters $a, v, s$ or $p$.

Vector and axial-vector correlators. The vector correlator certainly plays the most important role, mostly because it covers the processes induced by the photon. Already in 1979 the massless $\alpha_{s}^{2}$ corrections have been evaluated [60] and roughly ten years later even the order $\alpha_{s}^{3}$ corrections became available [39, 38]. However, due to the impressive experimental precision the massless approximations are not sufficient for a reliable comparison as we will see below in the case of the hadronic contribution of $\Delta \alpha$ (cf. Section 5.2.2). However, a complete analytical computation of $\Pi^{v}\left(q^{2}\right)$ at three-loop order is currently not feasible. In Ref. [134, 135] the Padé method described above has been applied and semi-numerical results have been obtained. At that time only the mass corrections of order $m^{2} / q^{2}$ [114], for which no asymptotic expansion has to be applied, have been available in the high-energy region. They have been combined with the terms up to order $\left(q^{2} / m^{2}\right)^{8}$ 135 in the small- $q^{2}$ expansion in order to get semi-analytical results for the individual colour structures.

As the main interest is in the imaginary part, one could also adopt the attitude to compute as many terms in the high-energy expansion as possible. Going, however, beyond the $m^{2}$ terms a naive expansion fails and the large-momentum procedure has to be applied. The calculation becomes very cumbersome if it has to be applied by hand. For this reason the large-momentum procedure has been automated and the program Imp [74] has been developed. As a first application correction terms up to order $\left(\mathrm{m}^{2} / q^{2}\right)^{6}$ have been evaluated [136, 137] for the vector correlator. In Fig. 5.2 the comparison of the individual expansion terms with the Padé result from Ref. 134 is shown.

For all three functions $R_{A}^{(2), v}, R_{N A}^{(2), v}$ and $R_{l}^{(2), v}$ and values between $x=0$ and $x=0.6$ $(x=2 \mathrm{~m} / \sqrt{s})$ the expansions including terms of order $\left(\mathrm{m}^{2} / \mathrm{s}\right)^{3}$ (or more) are in perfect agreement with the semi-analytical Padé result. Conversely this provides a completely independent test of the method of [134] which did rely mainly on low energy information. Including more terms in the expansion, one obtains an improved approximation even in the low energy region. However, the quality of the "convergence" is significantly better for 

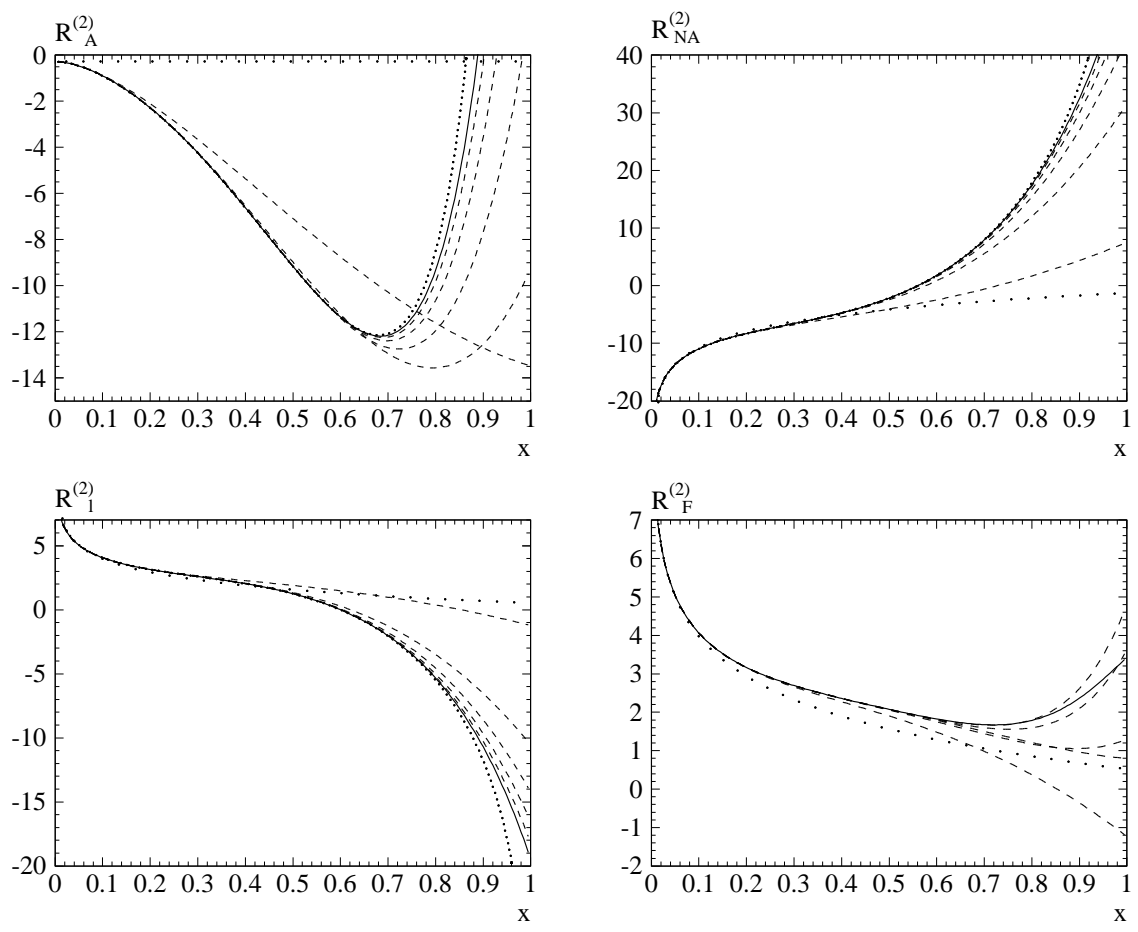

Figure 5.2: The abelian contribution $R_{A}^{(2), v}$, the non-abelian piece $R_{N A}^{(2), v}$, the contribution from light internal quark loops $R_{l}^{(2), v}$ and the contribution $R_{F}^{(2), v}$ from the double-bubble diagram with the heavy fermion in both the inner and outer loop as functions of $x=$ $2 \mathrm{~m} / \sqrt{s}$. Wide dots: no mass terms; dashed lines: including mass terms $\left(\mathrm{m}^{2} / \mathrm{s}\right)^{n}$ up to $n=5$; solid line: including mass terms up to $\left(\mathrm{m}^{2} / \mathrm{s}\right)^{6}$; narrow dots: semi-analytical result (except for $R_{F}^{(2), v}$ ). The scale $\mu^{2}=m^{2}$ has been adopted.

$R_{l}^{(2), v}$ and $R_{N A}^{(2), v}$ than for $R_{A}^{(2), v}$. Two reasons may be responsible for this difference: (i) In a high energy expansion it is presumably more difficult to approximate the $1 / v$ Coulomb singularity in $R_{A}^{(2), v}$ than the mild $\ln v$ singularity in $R_{N A}^{(2), v}$ and $R_{l}^{(2), v}$. (ii) The function $R_{l}^{(2), v}$ can be approximated in the whole energy region $2 m<\sqrt{s}<\infty$ by an increasing number of terms with arbitrary accuracy. This is evident from the known analytical form of $R_{l}^{(2), v}$ [112, a consequence of the absence of thresholds above $2 m$ in this piece. In contrast the functions $R_{A}^{(2), v}, R_{N A}^{(2), v}$ and $R_{F}^{(2), v}$ exhibit a four particle threshold at $\sqrt{s}=4 m$. The high energy expansion is, therefore, not expected to converge to the correct answer in the interval between $2 m$ and $4 m$. In particular, in the case of $R_{F}^{(2), v}$ it can be seen that for $x>0.5$ no convergence is observed.

At this point it is tempting to combine both approaches - asymptotic expansion in the high-energy region and the Padé method - and evaluate high-order Padé approximants. A detailed study in the case of the vector correlator can be found in [74 from which Fig. 5.3 is taken. In Fig. 5.3 the influence of the number of low- and high-energy input data is studied. In all four plots the quantity $\delta R_{A}^{(2)}=R_{A}^{(2), v}-3\left(\pi^{4} /(8 v)-3 \pi^{2}\right)$ is shown, 


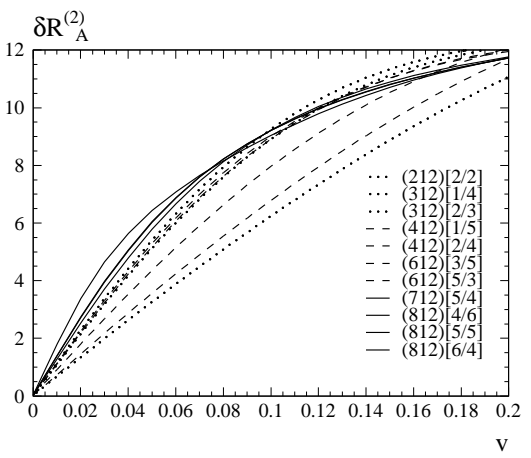

(a)

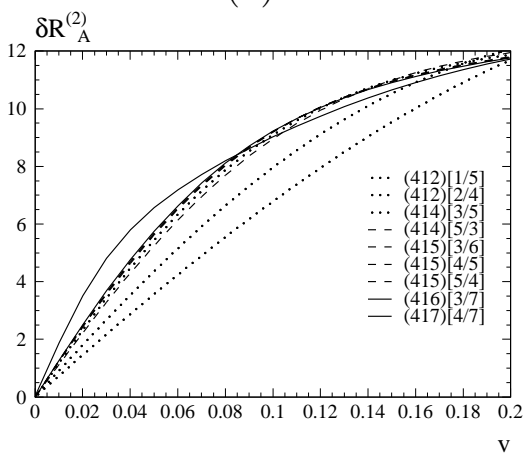

(c)

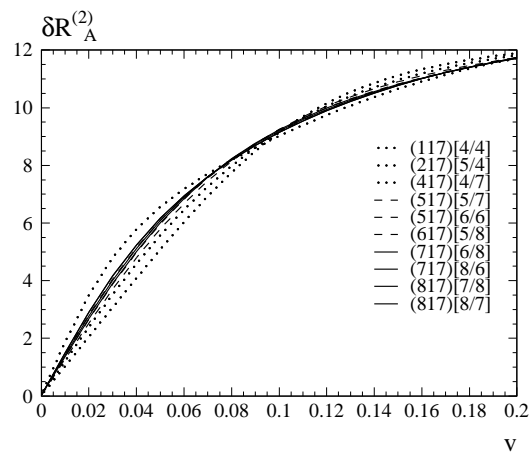

(b)

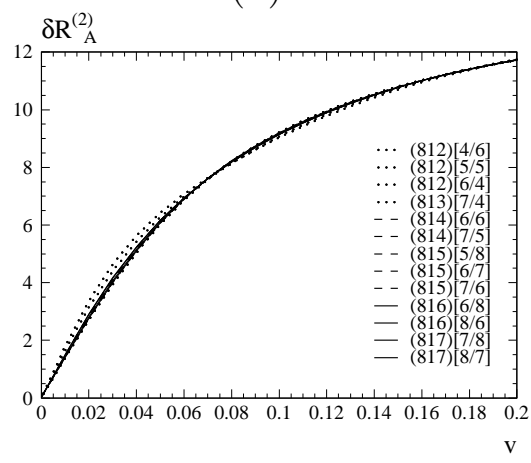

(d)

Figure 5.3: Abelian part of the vector correlator as a function of $v$. The leading threshold term is subtracted as described in the text. In (a) and (b) the number of low-energy moments are varied and the high-energy terms are fixed to two and seven, respectively. In (c) and (d) the low-energy moments are fixed to four and eight, respectively, and the high-energy terms are varied. The notation $(l 1 k)[i / j]$ means that terms up to order $z^{l}$ and $1 / z^{k-1}$ are taken into account in order to construct the Padé approximation $[i / j]$.

i.e. the leading threshold term is subtracted. Otherwise it would not be possible to detect any difference between the individual Padé results. Moreover, the abscissa only extends to $v=0.2$ as for $v \gtrsim 0.5$ all curves coincide. In Figs. 5.3(a) and 5.3(b) the higher-energy terms are fixed to two and seven, respectively, whereas an increasing number of low-energy moments are considered. On the other hand, on the plots in the lower row the number of moments is fixed to four and eight, respectively, with varying high-energy input.

From Figs. 5.3(a) and 5.3(c) a clear stablilization of the results can be observed with increasing degree of the Padé approximation. The same is true for Figs. 5.3(b) and 5.3(d): although the degree of the Padé approximants is higher from the very beginning a further stabilization is visible.

From these considerations one can conclude that both the small- and high-energy expansion terms are crucial as input for the Padé procedure. A significant stabilization of $R(s)$ in the threshold region is observed if more terms are taken into account. However, 
for practical purposes it is probably more than enough to consider, e.g., only the quadratic terms in the high-energy region. The reason for this is that also the leading threshold behaviour is incorporated into the analysis. The situation is different in those cases where no information about the threshold is available or one even wants to determine the value of the (real part of the) considered function for $z=1$. We will come back to this point in Section 5.4.

At this point we refrain from listing the results of the individual terms contributing to $R(s)$ as all of them are available in the literature. In particular we want to refer to the Appendix of Ref. [107] where detailed results up to order $\alpha_{s}^{2} m^{4} / s^{2}$ and $\alpha_{s}^{3} m^{2} / s$ are listed. Concerning the full mass dependence at order $\alpha_{s}^{2}$ a complete discussion and a detailed compilation of the individual terms in the on-shell scheme can be found in Ref. [127] (see also [138). Together with the quartic on-shell terms at order $\alpha_{s}^{3}$ given in Eq. (4.79) this constitutes the current state-of-the-art radiative corrections for $R(s)$.

In 135 next to the vector case also the axial-vector, scalar and pseudo-scalar correlators have been considered. Moments up to order $\left(q^{2}\right)^{8}$ have been combined with quadratic mass terms and the leading threshold behaviour in order to obtain semi-numerical approximations for the correlators.

QCD corrections to the axial-vector correlator have also been evaluated in [110]. In the limit $q^{2} \gg M^{2}$ the first seven terms could be evaluated using automated asymptotic expansion. In Fig. 5.4 the expansion terms are compared with the Padé results [135] for the individual colour factors. Again perfect agreement is found in the region where the asymptotic expansion is expected to converge to the exact result.

In [110] the results have been used in order to obtain in combination with the vector correlator, order $\alpha_{s}^{2}$ corrections to the top quark production in $e^{+} e^{-}$annihilation above the threshold. The results for the cross section are shown in Fig. 5.5 where also the electro-weak corrections have been included [139].

Scalar and Pseudo-scalar correlators. For completeness we want to mention that the high-energy expansions of the scalar and pseudo-scalar correlators have been considered in 95. The results have been applied to the decay of a scalar and pseudo-scalar Higgs boson into top quarks where higher order mass effects are important. This can be seen in Tab. 5.1 where a (pseudo-)scalar Higgs boson mass of $450 \mathrm{GeV}$ has been considerd. Both the results for the individual mass-correction terms $\left(M_{t}^{2} / M_{H}^{2}\right)^{i}(i=0, \ldots, 4)$ and their proper sum is listed up to order $\alpha_{s}^{2}$.

Singlet contribution. A special feature of the diagonal current correlator is the occurence of so-called singlet diagrams as pictured in Fig. 5.6. They are often also referred to as double-triangle diagrams as the external currents are not connected through the same fermion line. Note that for the vector correlator there are no singlet diagrams at three-loop level according to Furry's theorem [140]. In case of axial-vector couplings one has to take both members of a weak isospin doublet into account in order for the axial anomaly to cancel. It is therefore convenient to replace the current $j_{\mu}^{a}$ in Eq. (5.15) by $j_{S, \mu}^{a}=\bar{\psi} \gamma_{\mu} \gamma_{5} \psi-\bar{\chi} \gamma_{\mu} \gamma_{5} \chi$, where $\psi$ and $\chi$ are isospin partners. The diagrams contributing to the singlet part, $\Pi_{S}^{(2), a}\left(q^{2}\right)$, are depicted in Fig. 5.6 where in the fermion triangles either $\psi$ or $\chi$ may be present. Note that for a degenerate quark doublet $\Pi_{S}^{(2), a}\left(q^{2}\right)$ vanishes. 

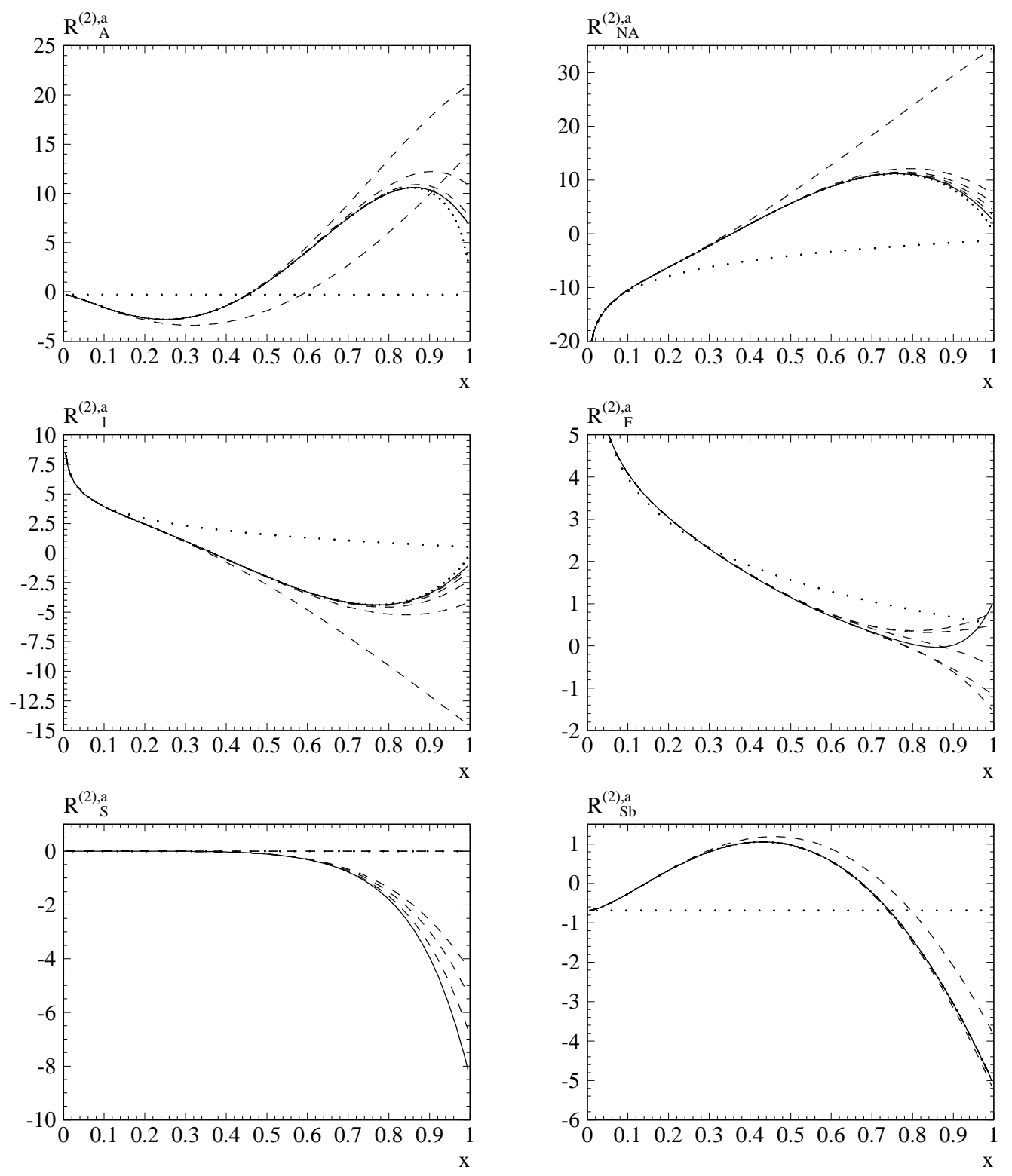

Figure 5.4: $\quad R_{i}^{(2), a}, i=A, N A, l, F, S, S b$ as functions of $x=2 M_{t} / \sqrt{s}$ at $\mu^{2}=M_{t}^{2}$. Successively higher order terms in $\left(M_{t}^{2} / s\right)^{n}$ are included: Dotted: $n=0$; dashed: $n=$ $1, \ldots, 5$; solid: $n=6$. Narrow dots: exact result $\left(R_{l}^{(2), a}, R_{S b}^{(2), a}\right)$ or semi-analytical results $\left(R_{A}^{(2), a}, R_{N A}^{(2), a}\right)$. In the case of the top-bottom doublet $R_{S b}$ contains only the imaginary parts of the singlet diagrams which arise from the gluon and bottom quark cuts (see also Fig. 5.7).

Having furthermore in mind the physical case $(\psi, \chi)=(t, b)$, it is justified to set $m_{\psi}=M$ and $m_{\chi}=0$. In the case of the scalar and pseudo-scalar currents there is no anomaly. Furthermore, as the coupling is proportional to the quark mass, only one diagram has to 
(a) Electroweak corrections

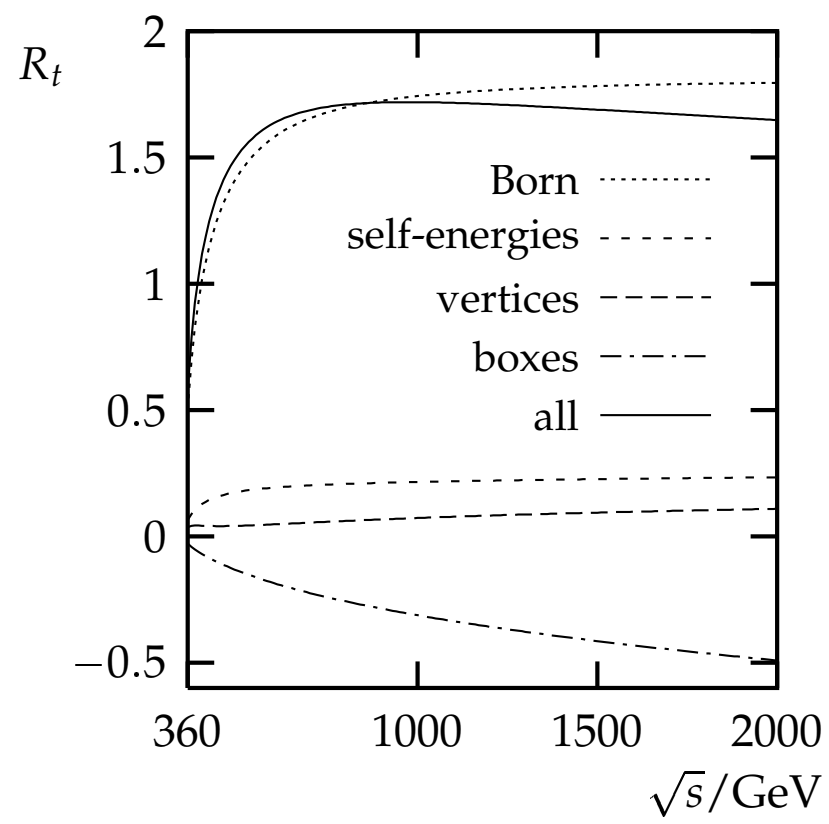

(b) QCD corrections

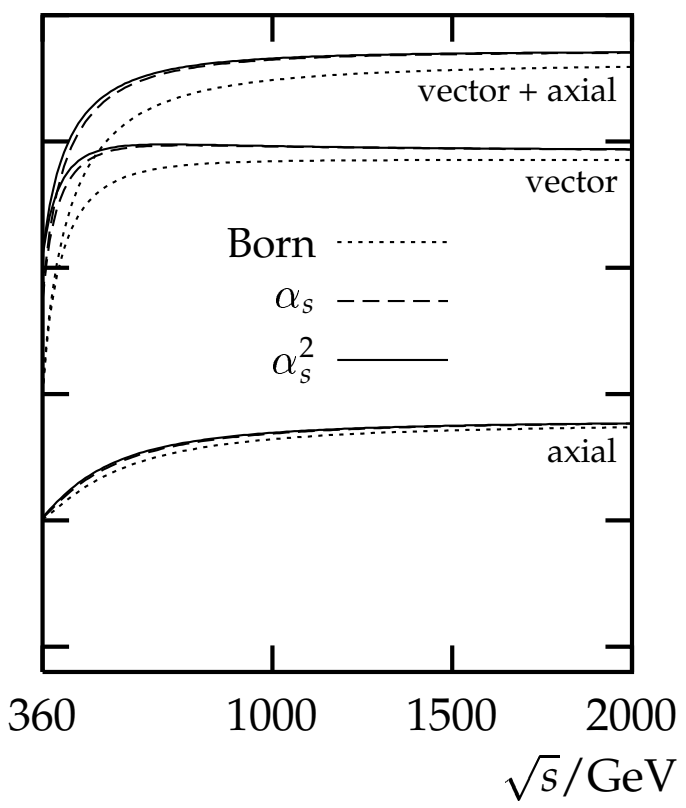

Figure 5.5: The electroweak (a) and QCD (b) corrections to $e^{+} e^{-} \rightarrow t \bar{t}$. In this Figure the normalized cross section $R_{t}=\sigma\left(e^{+} e^{-} \rightarrow t \bar{t}\right) / \sigma_{p t}$ is shown. $\sigma_{p t}=4 \pi \alpha^{2} / 3 s$.
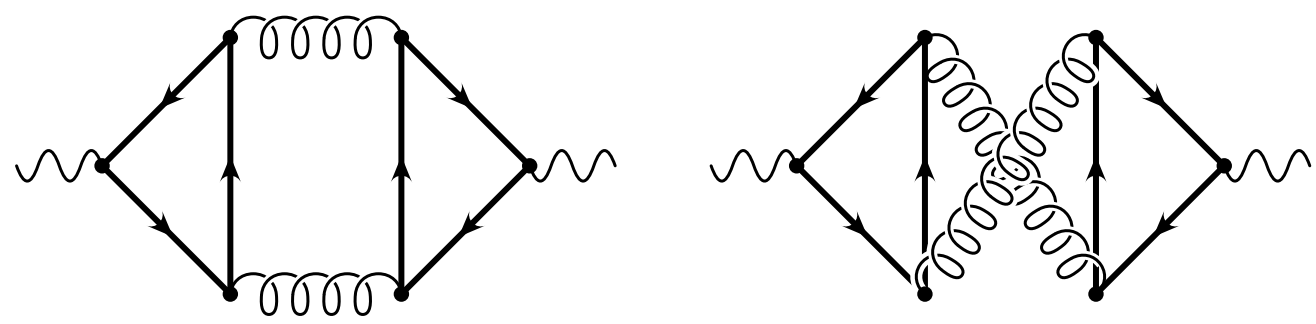

Figure 5.6: Singlet or double-triangle diagrams. In the fermion lines either the quark $\psi$ or its isospin partner $\chi$ may be present.

be considered, namely the one where in both quark lines the same heavy quark flavour is present.

As mentioned in Section 5.1 it is essential that the expansion for $q^{2} \rightarrow 0$ is analytical. However, in the case of the singlet diagrams this is not fulfilled as there exist massless cuts containing gluons and light fermions. Thus the method of Section 5.1 cannot directly be applied to $\Pi_{S}^{(2), \delta}\left(q^{2}\right)$. Rather it is applied to

$$
\begin{aligned}
& \Pi_{S, \text { mod }}^{(2), a}\left(q^{2}\right)=\Pi_{S}^{(2), a}\left(q^{2}\right)-\frac{1}{12 \pi^{2}} \int_{0}^{1} \mathrm{~d} r \frac{R_{S b}^{(2), a}(s)}{r-z}, \\
& \Pi_{S, \text { mod }}^{(2), \kappa}\left(q^{2}\right)=\Pi_{S}^{(2), \kappa}\left(q^{2}\right)-\frac{1}{8 \pi^{2}} \int_{0}^{1} \mathrm{~d} r \frac{R_{g g}^{(2), \kappa}(s)}{r-z}, \quad \kappa=s, p,
\end{aligned}
$$




\begin{tabular}{|c|c|c|c|c|c|c|c|}
\hline & $\left(M_{t}^{2}\right)^{0}$ & $\left(M_{t}^{2}\right)^{1}$ & $\left(M_{t}^{2}\right)^{2}$ & $\left(M_{t}^{2}\right)^{3}$ & $\left(M_{t}^{2}\right)^{4}$ & $\Sigma$ & exact \\
\hline & \multicolumn{5}{|c|}{ scalar, on-shell } & & \\
\hline$R^{(0), s} / 3$ & 1.000 & -0.907 & 0.137 & 0.014 & 0.003 & 0.247 & 0.248 \\
\hline$C_{F} R^{(1), s} / 3$ & -0.778 & 5.646 & -3.080 & 0.010 & 0.004 & 1.802 & 1.802 \\
\hline$R^{(2), s} / 3$ & -35.803 & 77.056 & -17.792 & -5.347 & -0.680 & 17.435 & - \\
\hline \multirow[t]{2}{*}{$\Sigma_{i}\left(\alpha_{s} / \pi\right)^{i}$} & 0.943 & -0.663 & 0.026 & 0.009 & 0.003 & 0.318 & \\
\hline & \multicolumn{5}{|c|}{ scalar, $\overline{\mathrm{MS}}$} & & \\
\hline$\frac{m_{t}^{2}}{M_{t}^{2}} \bar{R}^{(0), s} / 3$ & 0.784 & -0.558 & 0.066 & 0.005 & 0.001 & 0.298 & 0.299 \\
\hline$\frac{m_{t}^{2}}{M_{t}^{2}} C_{F} \bar{R}^{(1), s} / 3$ & 4.445 & -3.723 & -0.238 & 0.143 & 0.032 & 0.659 & 0.673 \\
\hline$\frac{m_{t}^{2}}{M_{t}^{2}} \bar{R}^{(2), s} / 3$ & 21.799 & -7.606 & -15.334 & 0.680 & 0.546 & 0.086 & - \\
\hline \multirow[t]{2}{*}{$\Sigma_{i}\left(\alpha_{s} / \pi\right)^{i}$} & 941 & -0.679 & 0.045 & 0.010 & 0.002 & 0.319 & \\
\hline & \multicolumn{5}{|c|}{ pseudo-scalar, on-shell } & & \\
\hline$R^{(0), p} / 3$ & 1.000 & -0.302 & -0.046 & -0.014 & -0.005 & 0.633 & 0.629 \\
\hline$C_{F} R^{(1), p} / 3$ & -0.778 & 3.495 & 0.417 & 0.047 & 0.027 & 3.208 & 3.238 \\
\hline$R^{(2), p} / 3$ & -35.803 & 25.024 & 12.173 & 3.780 & 1.293 & 6.467 & - \\
\hline \multirow[t]{2}{*}{$\Sigma_{i}\left(\alpha_{s} / \pi\right)^{i}$} & 0.943 & -0.172 & -0.022 & -0.009 & -0.003 & 0.737 & \\
\hline & \multicolumn{5}{|c|}{ pseudo-scalar, $\overline{\mathrm{MS}}$} & & \\
\hline$\frac{m_{t}^{2}}{M_{t}^{2}} \bar{R}^{(0), p} / 3$ & 0.784 & -0.186 & -0.022 & -0.005 & -0.002 & 0.569 & 0.569 \\
\hline$\frac{m_{t}^{2}}{M_{t}^{2}} C_{F} \bar{R}^{(1), p} / 3$ & 4.445 & -0.248 & -0.215 & -0.119 & -0.043 & 3.821 & 3.791 \\
\hline$\frac{m_{t}^{2}}{M_{t}^{2}} \bar{R}^{(2), p} / 3$ & 21.799 & 9.854 & 2.455 & -0.781 & -0.520 & 32.807 & - \\
\hline$\Sigma_{i}\left(\alpha_{s} / \pi\right)^{i}$ & 0.941 & -0.185 & -0.026 & -0.010 & -0.003 & 0.717 & \\
\hline
\end{tabular}

Table 5.1: Numerical results for $R^{s}$ and $R^{p}$ both in the on-shell and $\overline{\mathrm{MS}}$ schemes. The contributions from the mass terms $\left(M_{t}^{2}\right)^{i}$, their sum $(\Sigma)$ and, where available, the exact results are shown. $\Sigma_{i}\left(\alpha_{s} / \pi\right)^{i}$ is the sum of the 1-, 2- and 3-loop terms. The numbers correspond to $M_{t}=175 \mathrm{GeV}$ and $M_{H / A}=450 \mathrm{GeV}$. The renormalization scale $\mu^{2}$ is set to $s=M_{H / A}^{2}$.

where $R_{S b}^{(2), a}$ and $R_{g g}^{(2), \kappa}$ denote the contribution of these massless cuts to $R_{S}^{(2), a}$ and $R_{S}^{(2), \kappa}$, respectively. Thus by definition, $\Pi_{S, \text { mod }}^{(2), \delta}\left(q^{2}\right)$ has the same analytical properties as the non-singlet polarization functions. The notation already suggests that in the scalar and pseudo-scalar case there is only the cut through the two gluons. On the contrary, this cut is zero in the axial-vector case according to the Landau-Yang-Theorem 1141 and only cuts involving the massless quark contribute. The analytical expressions for $R_{S b}^{(2), a}$ and $R_{g g}^{(2), \kappa}$ can be found in [142] and [131], respectively. Expansions of the former are also listed in 131.

In 131] the asymptotic expansion has been applied to the singlet diagrams and terms 
up to $\left(M^{2} / q^{2}\right)^{6}$ in the high-energy region have been evaluated. For small external momentum moments up to order $z^{7}$ (axial-vector) and $z^{8}$ (scalar and pseudo-scalar) are available. This input was used to compute roughly 30 Padé approximants for each correlator. In Fig. 5.7 (a)-(c) the results for the imaginary part of $\Pi_{S}^{(2), a}, \Pi_{S}^{(2), s}$ and $\Pi_{S}^{(2), p}$ (solid lines), together with the first seven terms of the high energy expansion (dashed and dotted lines) are shown as functions of $x=2 m / \sqrt{s}$. Note, that in the displayed region, $0<x<1$, $\operatorname{Im} \Pi_{S}^{(2)}=\operatorname{Im} \Pi_{S, \text { mod }}^{(2)}$. Therefore, if one is interested, e.g., in (inclusive) production of the heavy quarks only, the corresponding massless cuts (depicted as dash-dotted lines) have to be subtracted. The resulting curves are shown in Fig. 5.7 (d).
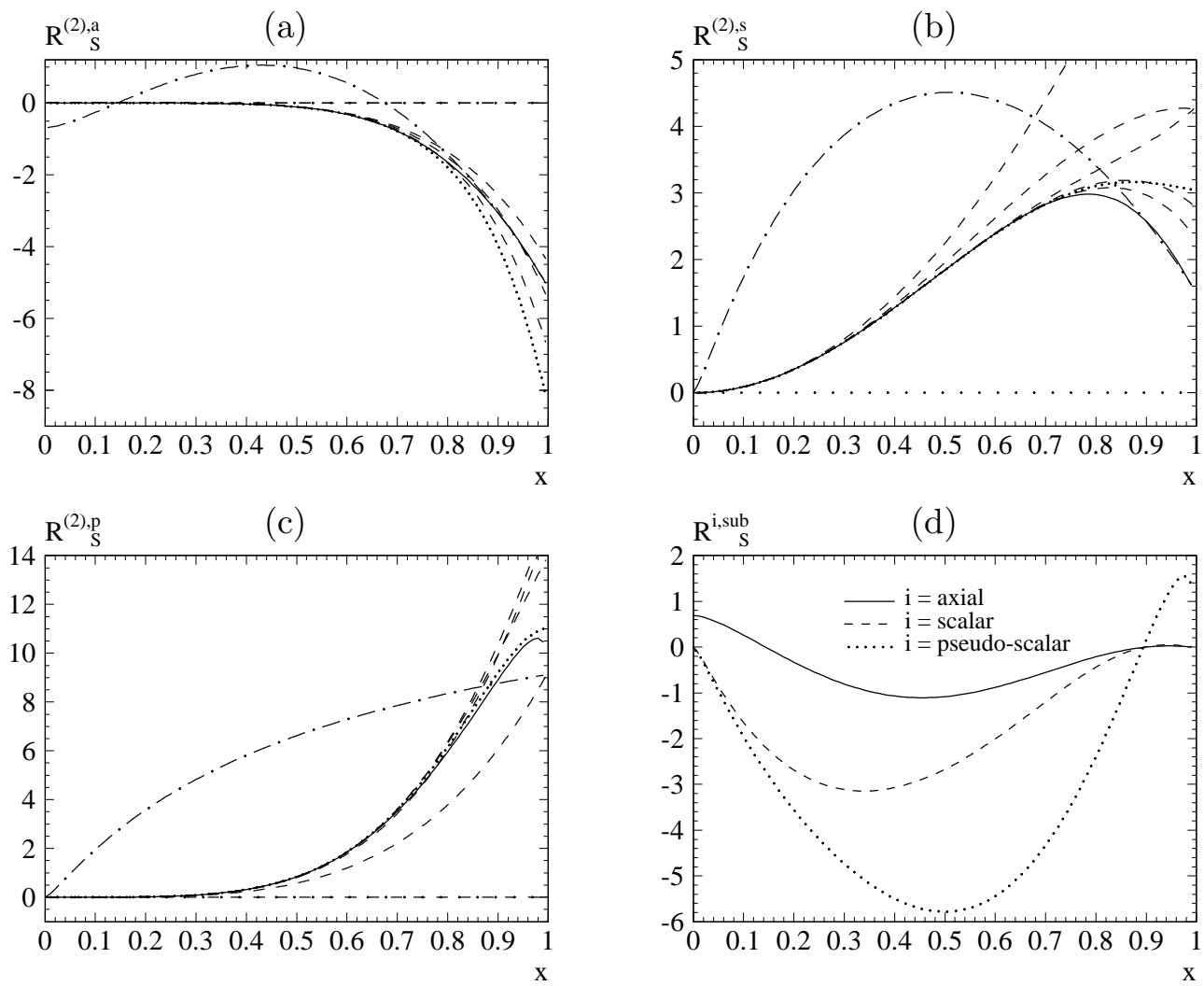

Figure 5.7: The imaginary parts $R_{S}^{(2), a}, R_{S}^{(2), s}$ and $R_{S}^{(2), p}$ of (a) the axial-vector, (b) scalar and (c) pseudo-scalar singlet diagrams, respectively. For the abscissa the variable $x=2 \mathrm{~m} / \sqrt{s}$ is chosen. Solid line: Padé result; wide dots, dashes and narrow dots: $\left(m^{2} / q^{2}\right)^{n}$-expansion for $n=0, n=1, \ldots, 5$ and $n=6$, respectively; dash-dotted line: purely massless cuts $R_{S b}^{(2), a}, R_{g g}^{(2), s}$ and $R_{g g}^{(2), p}$. Fig. (d) shows the difference between the solid and the dash-dotted line (i.e., the contribution of the massive quarks) of Figs. (a), (b) and (c) as solid, dashed and dotted line, respectively. The curves in (a) can also be found in the last row of Fig. 5.4.

The requirement that no logarithmic terms may appear in the expansion for $z \rightarrow 0$ 
(cf. Eq. (5.3) ) seems to be quite restrictive. However, the example of the singlet diagrams shows that also problems which at first sight do not match the definition of $f(z)$ in Eq. (5.3) can be treated.

From the systematic evaluation of the polarization function at low and high energies and the information about the leading threshold behaviour it is possible to construct - with the help of Padé approximation and conformal mapping - an approximation to $\Pi(z)$ taking into account the complete quark mass dependence. The imaginary part immediately leads to important physical quantities like $R(s)$, top quark pair production above threshold and the total decay rate of scalar and pseudo-scalar Higgs bosons.

\subsubsection{An application of $R(s): \Delta \alpha_{\text {had }}\left(M_{Z}\right)$}

An important application of the vector current correlator, as discussed in Sections 4.2 and 5.2.1, is the evaluation of the fine-structure constant at the scale of the $Z$ boson, $\alpha\left(M_{Z}^{2}\right)$. It plays a crucial role in the indirect determination of the Higgs boson mass which constitutes one of the most important goals of precision experiments. In particular, the error on $\alpha\left(M_{Z}^{2}\right)$ induces one of the largest uncertainties.

In Ref. [143] a conservative analysis has been performed which exclusively relies on data below a center-of-mass energy, $\sqrt{s}$, of $40 \mathrm{GeV}$. Consequently it suffers from sizable experimental errors. Only for $\sqrt{s} \geq 40 \mathrm{GeV}$ perturbative QCD has been used. Recently several suggestions have been made, which significantly reduce the uncertainties in $\alpha\left(M_{Z}^{2}\right)$. Most of them were actually triggered from the knowledge of the complete mass dependence at order $\alpha_{s}^{2}$ (see Section 5.2). There is a number of so-called theory-driven analyses [144, 145, 146, 147, 148, 149, 150 which replace to smaller and larger extent unprecise data by the results of perturbative QCD (pQCD). They will be discussed below.

Let us start with some basic definitions. Re-summation of the leading logarithms leads to

$$
\alpha(s)=\frac{\alpha(0)}{1-\Delta \alpha_{\text {lep }}(s)-\Delta \alpha_{\text {had }}^{(5)}(s)-\Delta \alpha_{\text {top }}(s)},
$$

with $\alpha=\alpha(0)=1 / 137.0359895$. $\Delta \alpha_{\text {lep }}$ denotes the leptonic contribution and is known up to the three-loop order [151]

$$
\Delta \alpha_{\text {lep }}\left(M_{Z}^{2}\right)=314.97686 \times 10^{-4} .
$$

Perturbation theory is also applicable to treat the contribution arising from the top quark. Including three-loop QCD corrections one gets [145]

$$
\Delta \alpha_{\text {top }}\left(M_{Z}^{2}\right)=(-0.70 \pm 0.05) \times 10^{-4} .
$$

The contribution from the remaining five quarks has to be taken into account using the dispersion integral

$$
\Delta \alpha_{\text {had }}^{(5)}\left(M_{Z}^{2}\right)=-\frac{\alpha M_{Z}^{2}}{3 \pi} \operatorname{Re} \int_{4 m_{\pi}^{2}}^{\infty} \mathrm{d} s \frac{R(s)}{s\left(s-M_{Z}^{2}-i \epsilon\right)},
$$




\begin{tabular}{|l|l|l|l|}
\hline \hline $\begin{array}{l}\Delta \alpha_{\text {had }}^{(5)}\left(M_{Z}^{2}\right) \\
\times 10^{4}\end{array}$ & $\alpha^{-1}\left(M_{Z}\right)$ & Reference & comment \\
\hline $273.2(4.2)$ & $128.985(58)$ & 152, Martin et al. '95 & (low-order) pQCD \\
$280(7)$ & $128.892(95)$ & 143, Eidelman et al. '95 & data \\
$280(7)$ & $128.892(95)$ & 153, Burkhardt et al. '95 & data \\
$275.2(4.6)$ & $128.958(63)$ & 154, Swartz '96 & - \\
$281.7(6.2)$ & $128.869(85)$ & 155, Alemany et al. '97 & $\tau$ data \\
$278.4(2.6)^{*}$ & $128.914(35)$ & 144, Davier et al. '97 & + pQCD \\
$277.5(1.7)$ & $128.927(23)$ & 145, Kühn et al. '98 & + CT \\
$277.6(4.1)$ & $128.925(56)$ & 146, Groote et al. '98 & SR (pQCD) \\
$277.3(2.0)^{* *}$ & $128.929(27)$ & 147, Erler '98 & $\tau$ data + UDR \\
$277.0(1.6)^{*}$ & $128.933(22)$ & 148, Davier et al. '98 & $\tau$ data + pQCD + SR \\
$277.8(2.5)$ & $128.922(34)$ & 149, Jegerlehner '99 & pQCD (ER) \\
$274.3(1.9)$ & $128.970(26)$ & 150, Martin et al. '00 & new data + pQCD (ER) \\
$276.1(3.6)$ & $128.946(49)$ & 156, Burkhardt et al. '01 & new data \\
$277.3(2.1)$ & $128.930(29)$ & 157, Jegerlehner '01 & pQCD (ER) + new data \\
\hline \hline
\end{tabular}

Table 5.2: Comparison of the different evaluations of $\Delta \alpha_{\mathrm{had}}^{(5)}\left(M_{Z}^{2}\right)$. The column "comment" reminds on the different methods used in the analysis as described in the text. $\left({ }^{*} \Delta \alpha_{\text {top }}\left(M_{Z}^{2}\right)\right.$ subtracted; ${ }^{* *}$ value corresponding to $\alpha_{s}\left(M_{Z}^{2}\right)=0.118$ adopted.)

with $R(s)=\sigma\left(e^{+} e^{-} \rightarrow\right.$ hadrons $) / \sigma\left(e^{+} e^{-} \rightarrow \mu^{+} \mu^{-}\right)$(cf. Eq. (5.19)). It is not possible to use perturbation theory for $R(s)$ in the whole energy region. Thus one has to rely to some extent on experimental results. The results of the recent evaluations can be found in Tab. 5.2 where also the resulting values for $\alpha^{-1}\left(M_{Z}^{2}\right)$ are listed. The latter has been obtained with the help of Eqs. (5.24), (5.25) and (5.26). In the fourth column of Tab. 5.2 some keywords are given which shall indicate the method used for the analysis. In the following we briefly describe the abbreviations. For more details we refer to the original papers.

$\tau$ data [155]. $\tau$ data from ALEPH have been used in order to get more information about $R(s)$ for energies below roughly $1.8 \mathrm{GeV}$. The hypothesis of conserved vector current (CVC) in combination with isospin invariance relates, e.g., the vector part of the two-pion $\tau$ spectral function to the corresponding part of the isovector $e^{+} e^{-}$cross section through the following relation

$$
\sigma^{I=1}\left(e^{+} e^{-} \rightarrow \pi^{+} \pi^{-}\right)=\frac{4 \pi \alpha^{2}(0)}{s} v_{J=1}\left(\tau \rightarrow \pi \pi^{0} \nu_{\tau}\right)
$$

A similar equation holds for the four-pion final state. Their incorporation into the analysis 
has been performed in [155] leading to a slight reduction of the error on $\Delta \alpha_{\text {had }}^{(5)}$.

Perturbative QCD (pQCD). The first attempt to replace unprecise data by pQCD can be found in 152. At that time, however, mass effects were barely known. Thus pQCD could only be applied far above the particle thresholds. Meanwhile $R(s)$ can be calculated in the framework of pQCD up to order $\alpha_{s}^{3}$ if quark masses are neglected [39, 38] and up to $\mathcal{O}\left(\alpha_{s}^{2}\right)$ with full quark mass dependence [112, 134, 135, 127]. In [144, 145] pQCD has been used down to an energy scale of $\sqrt{s}=1.8 \mathrm{GeV}$ and it has been shown that the non perturbative contributions are small. This leads to a further reduction of the error of about a factor two. For convenience we list in Tab. 5.3 the perturbative hadronic contributions for a variety of energy intervals. As our default values we adopt $\mu^{2}=s$, $\alpha_{s}^{(5)}\left(M_{Z}^{2}\right)=0.118$ [158], $M_{c}=1.6 \mathrm{GeV}$ and $M_{b}=4.7 \mathrm{GeV}$. In separate columns we list the variations with a change in the renormalization scale, the strong coupling constant and the quark masses:

$$
\delta \alpha_{s}= \pm 0.003, \quad \delta M_{c}= \pm 0.2 \mathrm{GeV}, \quad \delta M_{b}= \pm 0.3 \mathrm{GeV} .
$$

The typical contributions which have to be taken into account look as follows [145]. In the perturbative regions one receives contributions from light $(u, d$ and $s)$ quarks whose masses are neglected throughout, and from massive quarks which demand a more refined treatment. Below the charm threshold the light quark contributions are evaluated in order $\alpha_{s}^{3}$ plus terms of order $\alpha_{s}^{2} s /\left(4 M_{c}^{2}\right)$ from virtual massive quark loops. Above $5 \mathrm{GeV}$ the full $M_{c}$ dependence is taken into account up to order $\alpha_{s}^{2}$, and in addition the dominant cubic terms in the strong coupling are incorporated, as well as the corrections from virtual bottom quark loops of order $\alpha_{s}^{2} s /\left(4 M_{b}^{2}\right)$. Above $11.2 \mathrm{GeV}$ the same formalism is applied to the massive bottom quarks and charm quark mass effects are taken into account through their leading contributions in an $M_{c}^{2} / s$ expansion. All formulae are available for arbitrary renormalization scale $\mu$ which allows to test the scale dependence of the final answer. This was used to estimate the theoretical uncertainties from uncalculated higher orders. Matching of $\alpha_{s}$ between the treatment with $n_{f}=3,4$ and 5 flavours is performed at the respective threshold values (cf. Section 3). The influence of the small $\mathcal{O}\left(\alpha_{s}^{3}\right)$ singlet piece which prevents a clear separation of contributions from different quark species can safely be ignored for the present purpose.

Charm threshold region (CT) [145]. Perturbative QCD is clearly inapplicable in the charm threshold region between 3.7 and $5 \mathrm{GeV}$ where rapid variations of the cross section are observed. Data have been taken more than 15 years ago by the PLUTO 159, DASP [160], and MARK I collaborations [161]. The systematic errors of 10 to $20 \%$ exceed the statistical errors significantly and are reflected in a sizeable spread of the experimental results. In 145 the experimental data are normalized to match the predictions of perturbative QCD both below 3.7 and above $5.0 \mathrm{GeV}$. Two models have been constructed which describe the differences of the normalization factors below and above the considered energy interval.

\footnotetext{
${ }^{23}$ On the contrary, the inclusion of the $\tau$ data leads to a significant reduction of the error of the anomalous magnetic moment of the muon as it is more sensitive to the low-energy region.
} 


\begin{tabular}{|l|r|r|r|r|r|}
\hline \hline Energy range $(\mathrm{GeV})$ & central value & $\delta \mu$ & $\delta \alpha_{s}$ & $\delta M_{c}$ & $\delta M_{b}$ \\
\hline $1.800-2.125$ & 5.67 & 0.22 & 0.04 & 0.00 & 0.00 \\
$2.125-3.000$ & 11.66 & 0.21 & 0.06 & 0.01 & 0.00 \\
$3.000-3.700$ & 7.03 & 0.06 & 0.03 & 0.00 & 0.00 \\
$1.800-3.700$ & 24.36 & 0.48 & 0.13 & 0.01 & 0.01 \\
\hline $5.000-5.500$ & 5.44 & 0.03 & 0.03 & 0.06 & 0.00 \\
$5.500-6.000$ & 4.93 & 0.03 & 0.02 & 0.04 & 0.00 \\
$6.000-9.460$ & 25.45 & 0.11 & 0.08 & 0.10 & 0.00 \\
$9.460-10.520$ & 5.90 & 0.02 & 0.01 & 0.01 & 0.00 \\
$10.520-11.200$ & 3.48 & 0.01 & 0.01 & 0.00 & 0.00 \\
$5.000-11.200$ & 45.20 & 0.19 & 0.15 & 0.21 & 0.01 \\
$($ without $b \bar{b})$ & & & & & \\
\hline $11.200-11.500$ & 1.63 & 0.00 & 0.01 & 0.00 & 0.00 \\
$11.500-12.000$ & 2.62 & 0.00 & 0.01 & 0.00 & 0.00 \\
$12.000-13.000$ & 4.93 & 0.01 & 0.01 & 0.00 & 0.00 \\
$13.000-40.000$ & 72.92 & 0.08 & 0.12 & 0.02 & 0.02 \\
$12.000-40.000$ & 77.85 & 0.09 & 0.14 & 0.02 & 0.02 \\
$40.000-\infty$ & 42.67 & 0.03 & 0.06 & 0.00 & 0.00 \\
$11.200-\infty$ & 124.77 & 0.12 & 0.21 & 0.03 & 0.02 \\
\hline $1.8-\infty(\mathrm{pQCD})$ & 194.33 & 0.79 & 0.49 & 0.24 & 0.03 \\
\hline QED & 0.11 & - & - & - & - \\
\hline \hline
\end{tabular}

Table 5.3: Contributions to $\Delta \alpha_{\text {had }}^{(5)}\left(M_{Z}^{2}\right)$ (in units of $10^{-4}$ ) from the energy regions where pQCD is used (adopted from [127]). For the QED corrections the same intervals have been chosen. For the variation of $\alpha_{s}\left(M_{Z}^{2}\right), M_{c}$ and $M_{b}$ (cf. Eqs. (5.29)) have been used. $\mu$ has been varied between $\sqrt{s} / 2$ and $2 \sqrt{s}$.

Recently, the BES collaboration has measured $R(s)$ in the energy range between 2 and $5 \mathrm{GeV}$ with substantially improved precision [162]. We applied the method of [145] and obtained perfect agreement with the results of PLUTO, DASP and MARK I.

QCD sum rules (SR) [146, 148]. Global parton-hadron duality is used in order to reduce the influence of the data in the different intervals. This is achieved by choosing a proper polynomial, $Q_{N}(s)$, which is supposed to approximate the weight function $M_{Z}^{2} / s\left(s-M_{Z}^{2}\right)$ in Eq. (5.27) as good as possible. Adding and subtracting $Q_{N}(s)$ in Eq. (5.27) and exploiting the analycity of the subtracted term leads to

$$
\begin{aligned}
\int_{s_{0}}^{s_{1}} \mathrm{~d} s \frac{R(s) M_{Z}^{2}}{s\left(s-M_{Z}^{2}\right)}=\int_{s_{0}}^{s_{1}} \mathrm{~d} s R(s) & \left(\frac{M_{Z}^{2}}{s\left(s-M_{Z}^{2}\right)}-Q_{N}(s)\right) \\
& +6 \pi i \oint_{|s|=s_{1}} \mathrm{~d} s \Pi^{\mathrm{QCD}}(s) Q_{N}(s) .
\end{aligned}
$$

Thus the influence of the experimental data is significantly reduced in the first term of the right-hand side and pQCD only has to be used for $|s|=s_{1}$ which is indicated by the superscript QCD. 
Unsubtracted dispersion relations (UDR) [147]. They are used in order to evaluate the electromagnetic coupling in the $\overline{\mathrm{MS}}$ scheme. Four-loop running is accompanied by three-loop matching in order to arrive at $\bar{\alpha}\left(M_{Z}^{2}\right)$, which subsequently has to be transformed to the on-shell quantity $\alpha\left(M_{Z}^{2}\right)$. Via this method no complications in connection with the $J / \Psi$ or $\Upsilon$ resonances occur. However, one encounters a much stronger dependence on the quark masses.

Perturbative QCD in Euclidian region (ER) [149, 150, 157]. The authors of Ref. 1143 also re-evaluated $\Delta \alpha_{\text {had }}^{(5)}\left(M_{Z}\right)$ using pQCD. In a first step $\Delta \alpha_{\text {had }}^{(5)}$ was calculated at the large negative scale $s=-M_{Z}^{2}$ and then analytically continued to $s=M_{Z}^{2}$. Thus pQCD has only been applied in the Eulidian region where it is supposed to work best as one is far from resonances and thresholds. Furthermore pQCD has been used down to $-(2.5 \mathrm{GeV})^{2}$.

New data in the low-energy region. In the meantime new experimental data for $R(s)$ in the low-energy region have become available. Besides improvements in the energy interval below $1.4 \mathrm{GeV}$ by the CMD-2 detector at the VEPP-2M collider in Novosibirsk a measurement of $R(s)$ in the range $2 \mathrm{GeV}<\sqrt{s}<5 \mathrm{GeV}$ has been performed by the experiment BES II at Beijing. In Ref. [150] these data have been incorporated and accompanied with pQCD in the regions $3 \mathrm{GeV}<\sqrt{s}<3.74 \mathrm{GeV}$ and $\sqrt{s}>5 \mathrm{GeV}$ in order to evaluate $\Delta \alpha_{\text {had }}^{(5)}\left(M_{Z}\right)$. The result shown in Tab. 5.2 has been obtained using only inclusive measurements of $R(s)$ for $\sqrt{s} \lesssim 1.9 \mathrm{GeV}$. Based on the comparision of time-like and space-like (i.e. in the Euclidian region) evaluations of $\Delta \alpha_{\text {had }}^{(5)}\left(M_{Z}\right)$ it has been argued in 150 that this is preferred to the exclusive measurements of $R(s)$.

More recently the data-based analysis of [153 has been updated [156] using pQCD only above $\sqrt{s}=12 \mathrm{GeV}$. The main improvements are due to the new BES measurements.

Tab. 5.2 shows that the inclusion of pQCD leads to a significant reduction of the error in $\Delta \alpha_{\text {had }}^{(5)}\left(M_{Z}^{2}\right)$. The new analyses (with the exception of $\left[150 \|^{24}\right.$ ) agree well both in their central values and in their quoted errors. These promising developments suggest to use the new values in the interpretation of the electroweak measurements. Once more precise experimental results on $R$ are available it can replace the corresponding parts in the theory-motivated analyses. Certainly these measurements would be extremely valuable for a cross check of the theory-driven results.

\subsubsection{Heavy-light current correlators}

The method of Section 5.1 has also been applied to the non-diagonal correlators where one of the quark fields in Eq. (5.17) has mass $M$ and the other one is massless. In this limit the vector (scalar) and axial-vector (pseudo-scalar) correlators coincide. Furthermore it is convenient to work with the variables

$$
z=\frac{q^{2}}{M^{2}}, \quad x=\frac{M}{\sqrt{s}}, \quad v=\frac{1-x^{2}}{1+x^{2}} .
$$

${ }^{24}$ Actually, in 150 also a result based on exclusive data is given $\left(\Delta \alpha_{\text {had }}^{(5)}\left(M_{Z}^{2}\right)=(276.49 \pm 2.14) \times 10^{-4}\right)$ which is in better agreement with the other values. 
For the application of the Padé method the polarization function $\Pi^{\delta}(z)$ has to be considered in the different kinematical regions. In [163, 164] seven terms for small and eight terms for large external momentum have been computed both for the vector and scalar correlators of Eqs. (5.15) and (5.16), respectively. This means expansion coefficients up to order $z^{6}$, respectively, $1 / z^{7}$ are available for the Padé procedure.

The threshold behaviour constitutes the main difference as compared to the diagonal correlator discussed in the Section 5.2.1. The Born result for $R^{\delta}$ of the diagonal vector and pseudo-scalar correlators are proportional to $v$ for small velocities. At order $\alpha_{s}$ $R^{(1), \delta}$ approaches a constant and at order $\alpha_{s}^{2}$ one either has a $1 / v$ or a $\ln v$ behaviour depending on the colour structures [134, 135]. The axial-vetor and scalar correlators show the same pattern with a additional factor $v^{2}$ at each order.

On the contrary the imaginary part of the non-diagonal correlators are proportional to $v^{2}$ (possibly accompanied with $\ln v$ terms) independent of the order in $\alpha_{s}$ and of the colour structure. This is valid in every order in $\alpha_{s}$ as follows from Heavy Quark Effective Theory (HQET) [165]. Actually the latter can be used to obtain the leading threshold behaviour of $R^{v}(s)$ and $R^{s}(s)$ at $\mathcal{O}\left(\alpha_{s}^{2}\right)$ from the corresponding correlators in HQET. In particular, the renormalization group equation in the effective theory is used to get the leading logarithmic behaviour at order $\alpha_{s}^{2}$ [164]. Afterwards the decoupling relation between the currents in the full and effective theory [166, 167, 168] is exploited to get the information about $R^{v}(s)$ and $R^{s}(s)$ [163, 164]. It turns out that linear and quadratic logarithms occur. This translates into quadratic and cubic logarithms of the corresponding polarization function which are incorporated into the Padé method as descibed in step 3 of Section 5.1.

Before discussing the results in the full theory we want to spend time on the spectral function in HQET. In numerical form it reads 164]

$$
\begin{aligned}
\tilde{R}^{\prime}(\omega)= & N_{c} \omega^{2}\left[1+\frac{\alpha_{s}^{\left(n_{l}\right)}(\mu)}{\pi}\left(8.667+L_{\omega}\right)+\left(\frac{\alpha_{s}^{\left(n_{l}\right)}(\mu)}{\pi}\right)^{2}\left(\tilde{c}_{n_{l}}+35.54 L_{\omega}\right.\right. \\
& \left.\left.+1.875 L_{\omega}^{2}+n_{l}\left(-1.583 L_{\omega}-0.08333 L_{\omega}^{2}\right)\right)\right],
\end{aligned}
$$

where $\omega=\sqrt{s}-M$ is the only dimensionful quantity in the effective theory and $L_{\omega}=$ $\ln \left(\mu^{2} / \omega^{2}\right)$. The tilde and the prime remind that the quantity on the left-hand side of Eq. (5.32) is defined in the effective theory where the heavy quark is decoupled [167, 168]. In Eq. (5.32) the corrections of order $\alpha_{s}$ are known since quite some time 1169 whereas the constant $\tilde{c}_{n_{1}}$, which is not accessible using renormalization group techniques, has been determined in [164 with the help of the Padé results in the full theory. Its dependence on the number of massless quarks is given by

$$
\tilde{c}_{n_{l}}=46(15)-1.2(4) n_{l} .
$$

In the meantime the coefficient $\tilde{c}_{n_{l}}$ has been computed analytically [170] with the result $\tilde{c}_{n_{l}}=58-1.7 n_{l}$. Agreement with Eq. (5.33) can be observed for all physically reasonable values of $n_{l}$. 
We want to mention that $\tilde{R}^{\prime}$ enters as a building block into the sum rules which are used to determine the meson decay constants like, e.g., $f_{B}$ (see, e.g., Ref. [169). The typical scale where Eq. (5.32) has to be evaluated is $1 \mathrm{GeV}$. Thus the first order QCD corrections amount to about $100 \%$ and the terms of order $\alpha_{s}^{2}$ contribute with additional $60(20) \%$ where the sign is the same as for the LO correction.

In Figs. 5.8 and 5.9 the imaginary parts of the individual three-loop colour structures for the vector and scalar correlators are plotted as a function of the variable $x$. Each solid line contains of the order of 15 Padé approximants which show perfect agreement among each other. For comparison also the high-energy expansion terms including order $1 / z^{7}$ are shown as dashed curves. Excellent agreement with the semi-numerical Padé results is observed up to $x \approx 0.5$ which corresponds to $v \approx 0.60$. Some colour structures $\left(R_{l}^{(2), v}(s)\right.$, $\left.R_{A}^{(2), s}(s), R_{N A}^{(2), s}(s), R_{l}^{(2), s}(s)\right)$ show even an agreement up to $x \approx 0.7(v \approx 0.34)$ which is already fairly close to threshold. We want to remind that the functions which exhibit next to the cut at $\sqrt{s}=m$ also a cut at $\sqrt{s}=3 m$ are not expected to converge to the correct answer in the interval between $m$ and $3 m$. This explains the somewhat crazy behaviour of $R_{F}^{(2), s}(s)$ for larger values of $x$.

The non-diagonal correlators describe properties connected to the $W$ boson. In particular, a certain (gauge invariant) class of corrections to the Drell-Yan process, i.e. to the production of a quark pair through the decay of a virtual $W$ boson generated in $p \bar{p}$ collisions, are covered by the vector and axial-vector correlator. The absorptive part is directly related to the decay width of the (highly virtual) $W$ bosons into quark pairs and gluons. Of particular interest in this connection is the single-top-quark production via the process $q \bar{q} \rightarrow t \bar{b}$. The imaginary part of the transversal $W$ boson polarization function constitutes a finite and gauge invariant contribution of $\mathcal{O}\left(\alpha_{s}^{2}\right)$.

The corrections of order $\alpha_{s}$ to the (total) single-top-quark production rate are quite large. They amount to about 54\% and 50\% for Tevatron and LHC energies, respectively [171], where $18 \%$, respectively, $17 \%$ arise from the final state corrections. This calls for a complete $\mathcal{O}\left(\alpha_{s}^{2}\right)$ calculation.

If one considers the leading term of the large- $N_{c}$ limit it is possible to use the results for $R^{v}$ to perform a theoretical analysis of order $\alpha_{s}^{2}$ to the single-top-quark production. The production cross-section of the virtual $W^{*}$ boson is identical to that of the Drell-Yan process $q \bar{q} \rightarrow e \bar{\nu}_{e}$. The latter is known to $\mathcal{O}\left(\alpha_{s}^{2}\right)$ from Ref. [172. Thus we can take proper ratios to make predictions in the large- $N_{c}$ limit at NNLO free from any dependence on parton distribution functions. As an example, we consider

$$
\begin{aligned}
\frac{\frac{\mathrm{d} \sigma}{\mathrm{d} q^{2}}\left(p p \rightarrow W^{*} \rightarrow t b\right)}{\frac{\mathrm{d} \sigma}{\mathrm{d} q^{2}}\left(p p \rightarrow W^{*} \rightarrow e \nu_{e}\right)} & =\frac{\operatorname{Im}\left[\Pi_{t b}\left(q^{2}\right)\right]}{\operatorname{Im}\left[\Pi_{e \nu}\left(q^{2}\right)\right]} \\
& =N_{c}\left|V_{t b}\right|^{2} R^{v}(s) .
\end{aligned}
$$

The numerical significance of the order $\alpha_{s}^{2}$ corrections is shown in Fig. 5.10 where the LO, NLO and NNLO result of $R^{v}(s)$ is plotted in the range $\sqrt{s}=200 \ldots 400 \mathrm{GeV}$. For the numerical values $M_{t}=175 \mathrm{GeV}$ and $\alpha_{s}\left(M_{Z}\right)=0.118$ has been chosen. Whereas the $\mathcal{O}\left(\alpha_{s}\right)$ corrections are significant there is only a moderate contribution from the order $\alpha_{s}^{2}$ 

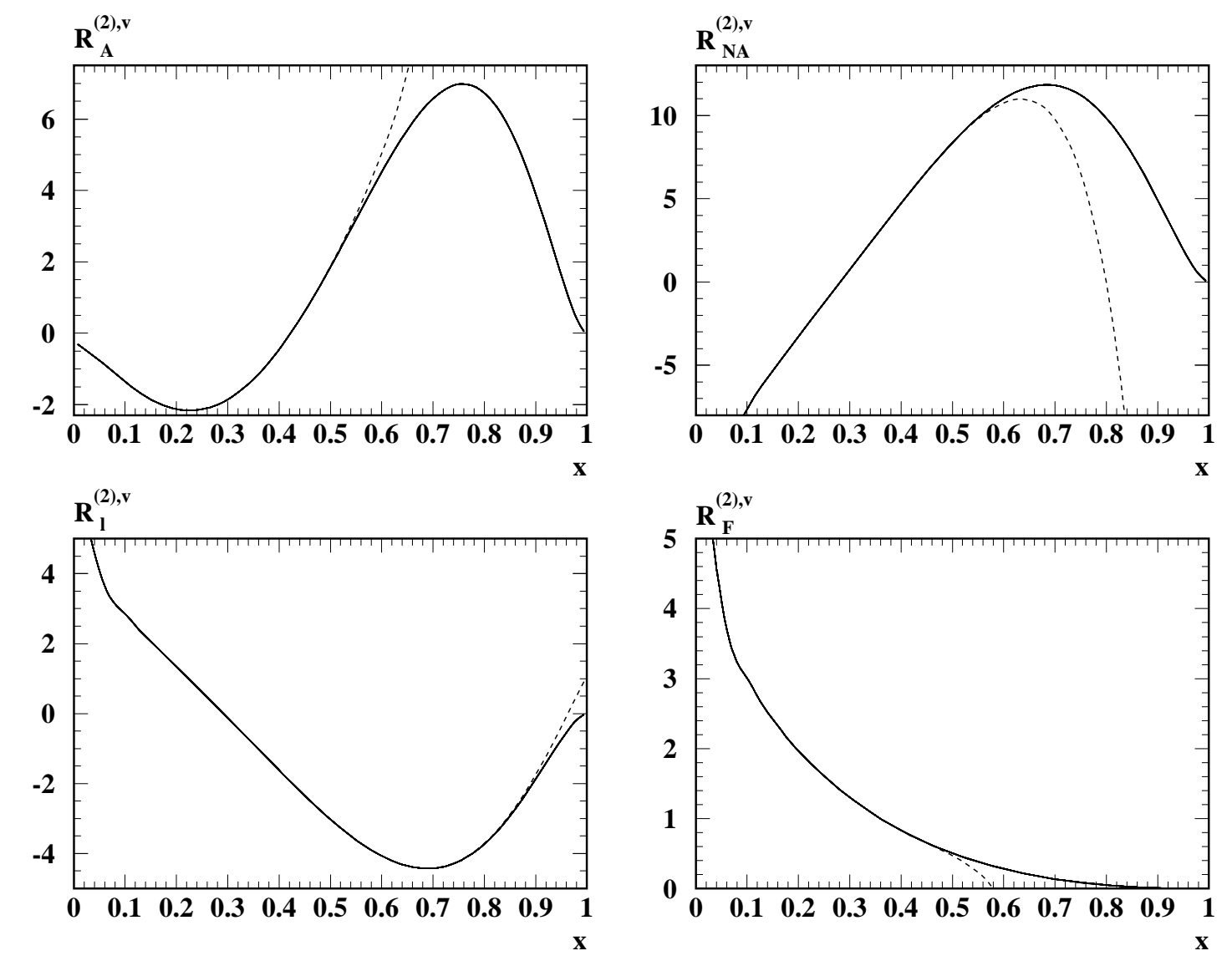

Figure 5.8: $R_{A}^{(2), v}(s), R_{N A}^{(2), v}(s), R_{l}^{(2), v}(s)$ and $R_{F}^{(2), v}(s)$ as a function of $x$. The dashed curves correspond to the analytical expressions obtained via asymptotic expansion containing the terms up to order $1 / z^{7}$.

terms. In the range in $q^{2}$ shown in Fig. 5.10 they are below $1 \%$ of the Born result. Note that (at least for $\mu^{2}=M_{Z}^{2}$ ) the NNLO corrections to the Drell-Yan process are also small and amount to at most a few percent (see e.g. [173]).

As an application of the scalar and pseudo-scalar current correlator we want to mention the decay of a charged Higgs boson which occurs in extensions of the Standard Model. The corrections to $R^{s}$ describe the total decay rate into a massive and a massless quark. To be more precise, the hadronic decay rate of the charged Higgs boson takes the form

$$
\Gamma\left(H^{+} \rightarrow U \bar{D}\right)=\frac{\sqrt{2} G_{F}}{8 \pi} M_{H^{+}}\left(a^{2}+b^{2}\right) R^{s}\left(M_{H^{+}}^{2}\right),
$$

where $a$ and $b$ parametrize the coupling of the Higgs boson to the massive quark $U$ and the massless quark $D$

$$
J_{H^{+}}=\frac{m_{U}}{\sqrt{2}} \bar{U}\left[a\left(1-\gamma_{5}\right)+b\left(1+\gamma_{5}\right)\right] D .
$$



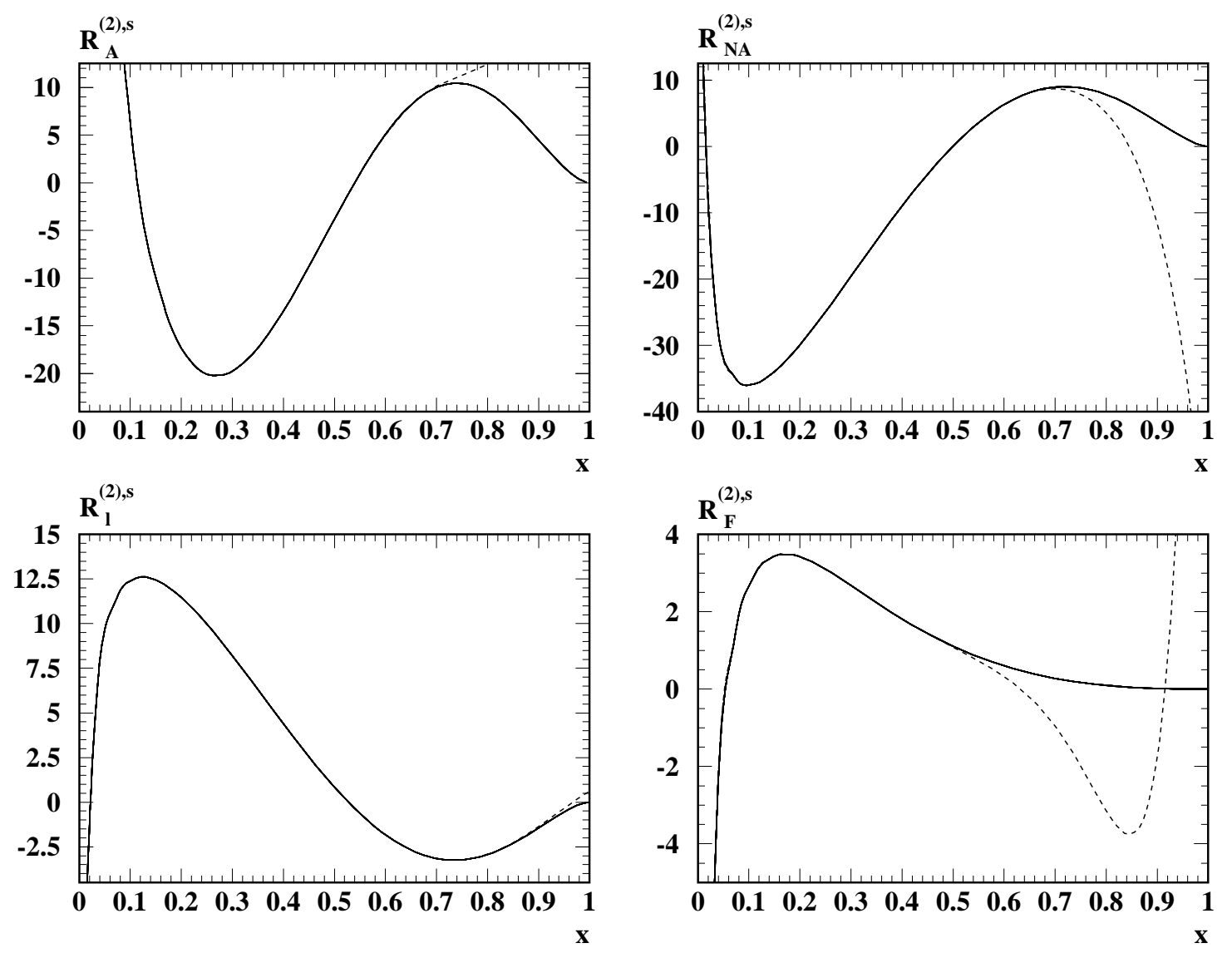

Figure 5.9: $R_{A}^{(2), s}(s), R_{N A}^{(2), s}(s), R_{l}^{(2), s}(s)$ and $R_{F}^{(2), s}(s)$ as a function of $x$. The dashed curves correspond to the analytical expressions obtained via asymptotic expansion containing the terms up to order $1 / z^{7}$.

In Fig. 5.11 $R^{s}(s)$ is plotted at LO, NLO and NNLO 163, 164. Again it turns out that the radiative corrections are well under control as order $\alpha_{s}^{2}$ terms contribute at most of the order of $1 \%$.

\subsection{QED corrections to muon decay}

The Fermi coupling constant, $G_{F}$, constitutes together with the electromagnetic coupling constant and the mass of the $Z$ boson the most precise input parameters of the SM of elementary particle physics. $G_{F}$ is defined through the muon lifetime which in turn is obtained from the decay rate. The one-loop corrections of order $\alpha$ have been evaluated more than 40 years ago in [17]. Only recently the two-loop corrections of order $\alpha^{2}$ have been computed by two independent groups [18, 19]. The inclusion of the new terms leads to the following value for the Fermi coupling constant [174]

$$
G_{F}=1.16639(1) \times 10^{-5} \mathrm{GeV}^{-2},
$$




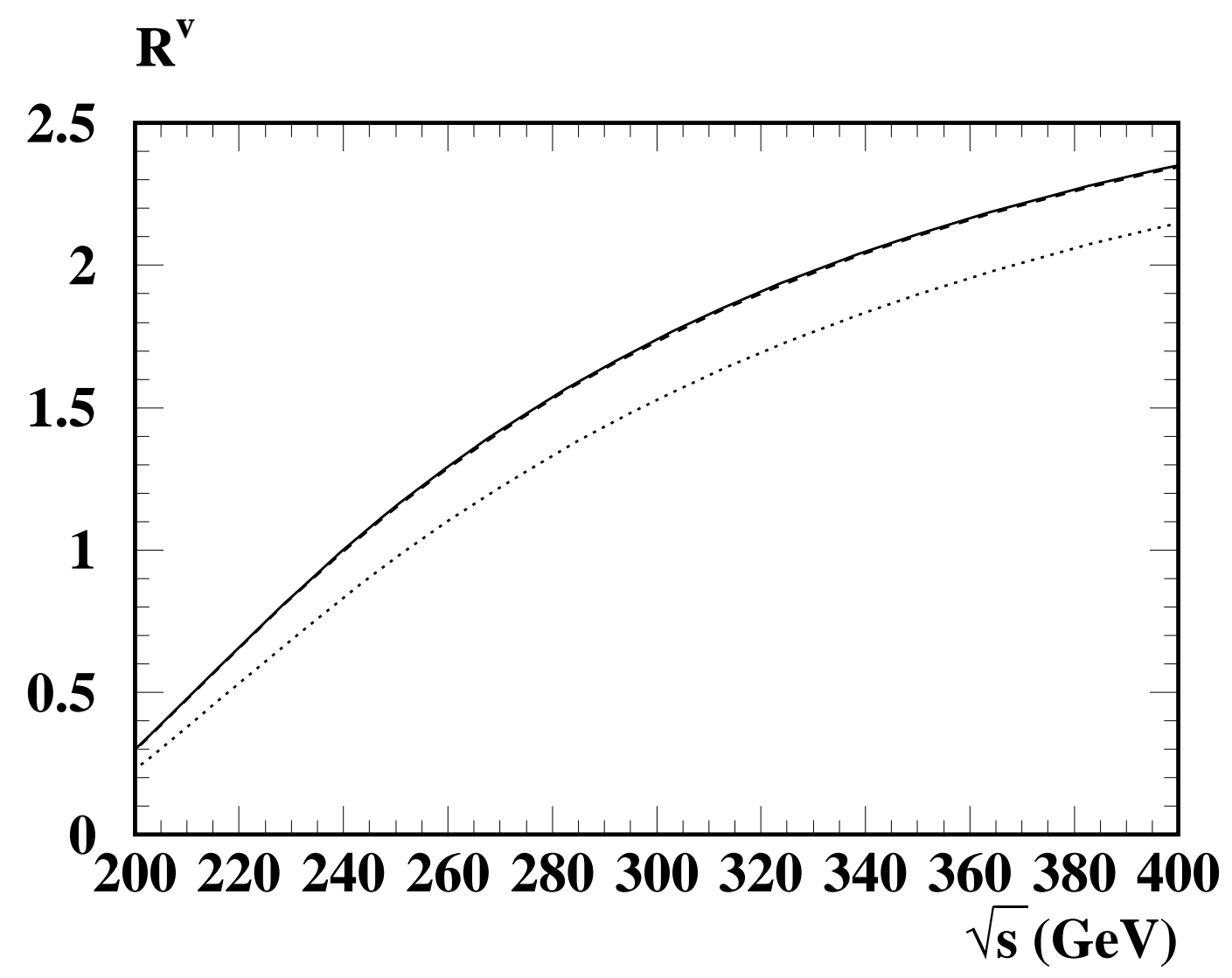

Figure 5.10: LO (dotted), NLO (dashed) and NNLO (solid) results of $R^{v}(s)$.

where the error is reduced by a factor two. It is now entirely experimental. In this Subsection we want to concentrate on the method used in [19] which is based on Padé approximation and conformal mapping. We also want to mention that in Ref. [175] optimization methods have been used in order to estimate the coefficient of order $\alpha^{3}$.

It is common to both calculations of the order $\alpha^{2}$ corrections that the muon propagator is considered in the framework of the effective theory where the $W$ boson is integrated out. The QED corrections to the resulting Fermi contact interaction were shown to be finite to all orders [176]. It is quite advantageous to perform a Fierz transformation which for a pure $V-A$ theory has the consequence that afterwards the two neutrino lines appear in the same fermion trace. Thus the QED corrections only affect the fermion trace involving the muon and the electron. This also provides some simplifications in the treatment of $\gamma_{5}$ since in the case of vanishing electron mass a fully anticommuting prescription can be used. 


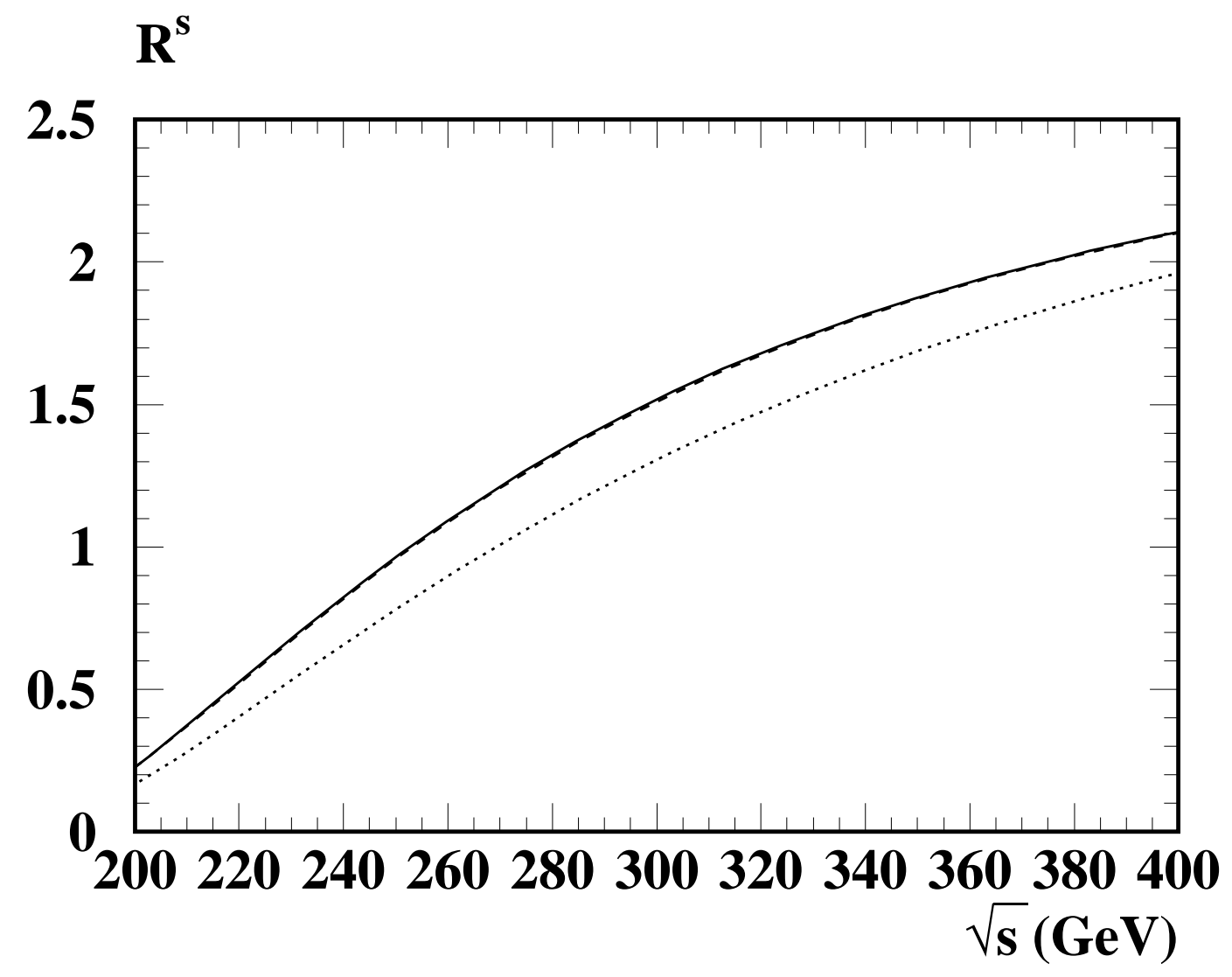

Figure 5.11: LO (dotted), NLO (dashed) and NNLO (solid) results of $R^{s}(s), M=M_{t}=$ $175 \mathrm{GeV}$.

The decay rate can be written in the form民2

$$
\Gamma=\left.2 M \operatorname{Im}\left[z S_{V}^{\mathrm{OS}}-S_{S}^{\mathrm{OS}}\right]\right|_{z=1},
$$

where

$$
S_{S}^{\mathrm{OS}}=Z_{2}^{\mathrm{OS}} Z_{m}^{\mathrm{OS}}\left(1-\Sigma_{S}^{0}\right), \quad S_{V}^{\mathrm{OS}}=Z_{2}^{\mathrm{OS}}\left(1+\Sigma_{V}^{0}\right),
$$

are functions of the variable

$$
z=\frac{q^{2}}{M_{\mu}^{2}} .
$$

$\Sigma_{S}^{0}$ and $\Sigma_{V}^{0}$ represent the scalar and vector part of the muon propagator. They are functions of the external momentum $q$ and the bare mass $m^{0}$. In our case they further depend on the bare electromagnetic coupling $\alpha^{0}$ and are proportional to the square of the Fermi coupling constant, $G_{F}^{2}$. Typical diagrams contributing to $\Sigma_{S}^{0}$ and $\Sigma_{V}^{0}$ are depicted

\footnotetext{
${ }^{25}$ Some discussion of Eq. (5.38) - in particular the additional factor $z$ in front of $S_{V}^{\mathrm{OS}}$ - can be found in 177 .
} 


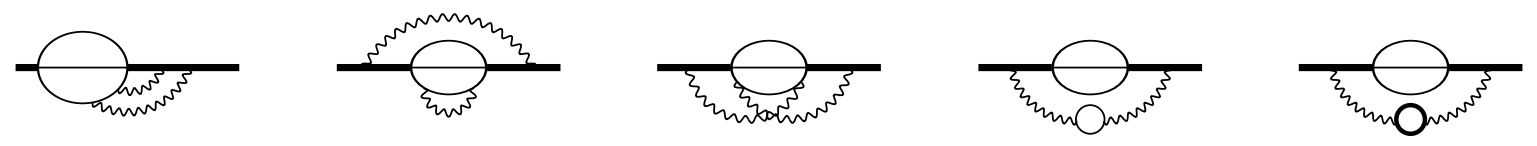

Figure 5.12: Sample diagrams for the muon (thick line) self energy. Two of the thin lines represent the electron and the corresponding neutrino and the third one the muon neutrino. All (one-particle-irreducible) diagrams involving the coupling of the photon (wavy lines) to the muon and electron have to be taken into account.

in Fig. 5.12. $Z_{2}^{\mathrm{OS}}$ and $Z_{m}^{\mathrm{OS}}$ represent the wave function and mass renormalization in the on-shell scheme.

It is convenient to parameterize the QED corrections for the muon decay in the following form

$$
\Gamma\left(\mu \rightarrow \nu_{\mu} e \bar{\nu}_{e}\right)=\Gamma_{\mu}^{0}\left[A_{\mu}^{(0)}+\frac{\bar{\alpha}}{\pi} A_{\mu}^{(1)}+\left(\frac{\bar{\alpha}}{\pi}\right)^{2}\left(A_{\mu}^{(2)}+A_{\mu, \tau}^{(2)}+A_{\mu, \mathrm{had}}^{(2)}\right)+\ldots\right],
$$

with $\Gamma_{\mu}^{0}=G_{F}^{2} M_{\mu}^{5} /\left(192 \pi^{3}\right) . \quad \bar{\alpha}=\bar{\alpha}(\mu)$ represents the electromagnetic coupling in the $\overline{\mathrm{MS}}$ scheme and $A_{\mu, \tau}^{(2)}$ corresponds to the contribution involving a virtual $\tau$ loop. It is suppressed by $M_{\mu}^{2} / M_{\tau}^{2}$ and almost four orders of magnitudes smaller than the other terms [178. The hadronic contribution is denoted by $A_{\mu, \mathrm{had}}^{(2)}[178]$.

To lowest order the result is known for finite electron mass, $M_{e}$

$$
A_{\mu}^{(0)}=1-8 \frac{M_{e}^{2}}{M_{\mu}^{2}}-12 \frac{M_{e}^{4}}{M_{\mu}^{4}} \ln \frac{M_{e}^{2}}{M_{\mu}^{2}}+8 \frac{M_{e}^{6}}{M_{\mu}^{6}}-\frac{M_{e}^{8}}{M_{\mu}^{8}},
$$

and in the limit $M_{e}=0$ we obtain for the one-loop corrections

$$
A_{\mu}^{(1)}=\frac{25}{8}-\frac{\pi^{2}}{2} \approx-1.810
$$

The approach chosen in [18] to evaluate $A_{\mu}^{(2)}$ has some similarity to the computation of the four-loop contribution to the $\beta$ function [14] (see also Section 2.3). In fact, since only the imaginary part has to be calculated one is only interested in the pole part like in the case of a $\overline{\mathrm{MS}}$ renormalization constant. However, only the pole part arising from the cuts through the electron and neutrino lines have to be taken into account and not the ones through the muon line. This is ensured by considering the on-shell muon propagator which requires the evaluation of (the imaginary part of) four-loop on-shell integrals with external momentum $q^{2}=M_{\mu}^{2}$. Integration-by-parts relations 477 are applied to reduce the number of occuring integrals to a small set of so-called master integrals. Only for the latter a hard calculation is necessary. Usually a large number of terms is generated in intermediate steps while using integration-by-parts relations. However, those four-loop integrals that have no imaginary part can immediately be discarded. This includes fourloop vacuum graphs and diagrams with a through-going on-shell line. A comprehensive discussion and lots of intermediate results can be found in Ref. [18]. 
The approach of 19 used for the computation of the QED corrections of order $\alpha^{2}$ is based on an expansion of the full fermion propagator in the limit $M_{\mu}^{2} \gg q^{2}$, where $q$ is the external momentum and $M_{\mu}$ is the on-shell propagator mass of the muon. The on-shell limit $q^{2} \rightarrow M^{2}$ will be performed afterwards with the help of Padé approximations. This, of course, only provides an approximation to the exact result. However, the integrals to be evaluated are simplified considerably. Furthermore, the accuracy obtained with this method is sufficient to check the result of [18 and enables the same reduction of the theoretical error on $G_{F}$. Good convergence to the exact result is also expected from the analysis performed in [179 where the hard-mass procedure has been applied to scalar two-loop integrals involving massless thresholds.

Due to the Fierz transformation the loop integration connected to the two neutrino lines can be performed immediately as it constitutes a massless two-point function. This is also the case after allowing for additional photonic corrections. As a result one encounters in the resulting diagram a propagator with one of the momenta raised to power $\varepsilon$ where $D=4-2 \varepsilon$ is the space-time dimension. This slightly increases the difficulty of the computation of the resulting diagrams. Especially for the order $\alpha^{2}$ corrections, where the original four-loop diagrams are reduced to three-loop ones with non-integer powers of denominators, it is a priori not clear that these integrals can be solved analytically. However, it turns out that for the topologies needed in our case this is indeed possible. For the computation of the massless two-point functions we have used the package MINCER [31]. Only slight modifications are necessary in order to use this package also for the computation of the new type of integrals.

In contrast to the current correlators considered in Section 5.2 the expansion terms for $z \rightarrow-\infty$ can not be used as they describe the unphysical process $\mu \rightarrow \mu+\gamma$. Another difference is the presence of massless cuts in the limit $q^{2} \rightarrow 0$. Thus a naive expansion is not possible and rather the asymptotic expansion has to be applied, which generates from the 44 contributing four-loop diagrams 72 sub- and cosub-diagrams that have to be evaluated. The analytical results are rather lengthly and cannot be listed. Instead the results are presented in numerical form [19].

In order to get reliable results it is necessary to compute as many terms as possible in the expansion parameter $z$. Subsequently a Padé approximation is applied as described in Section 5.1. We want to recall that before the Padé procedure a conformal mapping can be used which maps the complex $z$-plane into the interiour of the unit circle. Following Ref. [177] we denote those results by $\omega$-Padés and the ones obtained without conformal mapping by $z$-Padés.

The calculation is performed with the help of the package GEFICOM [41]. It uses QGRAF [57] for the generation of the diagrams and EXP [180] for the application of the asymptotic expansion procedures.

The method has been successfully tested at Born level and at order $\alpha$ where a large number of moments can be evaluated. This gives a hint on how of many terms are necessary at $\mathcal{O}\left(\alpha^{2}\right)$ in order to obtain a reliable answer. The results for $A_{\mu}^{(2)}$ of the 


\begin{tabular}{|l|l||c|c|}
\hline input & P.A. & $z$ & $\omega$ \\
\hline 6 & {$[3 / 2]$} & 5.836 & 7.249 \\
6 & {$[2 / 3]$} & 5.836 & 7.057 \\
\hline 7 & {$[4 / 2]$} & 5.935 & 7.040 \\
7 & {$[3 / 3]$} & 5.833 & 7.076 \\
7 & {$[2 / 4]$} & 5.938 & 7.080 \\
\hline 8 & {$[4 / 3]$} & 6.110 & 6.873 \\
8 & {$[3 / 4]$} & 6.113 & 7.060 \\
\hline \multicolumn{3}{|c||}{ exact: } & \multicolumn{2}{|c|}{6.743} \\
\hline
\end{tabular}

Table 5.4: Padé results for the corrections of $\mathcal{O}\left(\alpha^{2}\right)$ to the muon decay, $A_{\mu}^{(2)}$. The first row indicates the order in $z$ which has been used to construct the Padé approximations.

individual Padé approximations are shown in Tab. 5.4. It leads to the final answer [19]

$$
A_{\mu}^{(2)}=6.5(7)
$$

where the deviation of the central value from the exact result of 6.743 is less than $3 \%$ and well covered by the extracted error of roughly 10\%. Thus the sole knowledge of our results would also reduce the theoretical error on $G_{F}$. Finally, the total decay rate of the muon takes the form

$$
\Gamma\left(\mu \rightarrow \nu_{\mu} e \bar{\nu}_{e}\right)=\Gamma_{\mu}^{0}\left[0.9998-1.810 \frac{\bar{\alpha}\left(M_{\mu}\right)}{\pi}+6.700(2)\left(\frac{\bar{\alpha}\left(M_{\mu}\right)}{\pi}\right)^{2}+\ldots\right]
$$

where $A_{\mu, \text { had }}^{(2)}=-0.042(2)$ [178 and $A_{\mu, \tau}^{(2)}=-0.00058$ [178 has been used. Furthermore $\mu^{2}=M_{\mu}^{2}$ has been adopted.

As already noted in 175 the numerical coefficient in front of the second order corrections becomes very small ${ }^{20}$ if one uses the on-shell scheme for the definition of the coupling constant $\alpha$. Then the $\overline{\mathrm{MS}}$ coupling is given by $\bar{\alpha}\left(M_{\mu}\right)=\alpha\left(1+\alpha /(3 \pi) \ln \left(M_{\mu}^{2} / M_{e}^{2}\right)\right)$ and there is an accidental cancellation between the constant and the logarithm in the second order corrections.

A similar kinematical situation as in the $\mu$ decay is also given for the semileptonic decay of a bottom quark, $b \rightarrow u e \nu_{e}$. From the technical point of view the difference is only due to the non-abelian structure of QCD. The two methods described above have been applied to the total rate

$$
\Gamma\left(b \rightarrow u e \bar{\nu}_{e}\right)=\Gamma_{b}^{0}\left[1-2.413 \frac{\alpha_{s}\left(M_{b}\right)}{\pi}+A_{b}^{(2)}\left(\frac{\alpha_{s}\left(M_{b}\right)}{\pi}\right)^{2}+\ldots\right],
$$

where $\Gamma_{b}^{0}=G_{F}^{2} M_{b}^{5}\left|V_{u b}\right|^{2} /\left(192 \pi^{3}\right)$. The order $\alpha_{s}^{2}$ results read $A_{b}^{(2)}=-21.296$ 181 and $A_{b}^{(2)}=-21.1(6)$ 19. Again perfect agreement between the two methods is observed.

\footnotetext{
${ }^{26}$ Instead of " $6.7 "$ one has " $0.27 "$ in Eq. (5.45).
} 
At the end of this Subsection we want to mention that similar methods have been used to compute the decay rate of a top quark into a $W$ boson and a bottom quark 177. Contrary to the case of the muon the $W$ boson is not integrated out from the Lagrangian. Thus, at order $\alpha_{s}^{2}$ three-loop diagrams contributing to the top quark propagator have to be considered. In [177] a double expansion in $q^{2} / M_{t}^{2}$ and $M_{W}^{2} / M_{t}^{2}$ has been performed, where $q$ is the external momentum of the top quark propagator, which leads to a reliable prediction for $\Gamma(t \rightarrow W b)$ including finite $W$-mass effects.

\subsection{The relation between the $\overline{\mathrm{MS}}$ and on-shell quark mass}

In this Subsection we consider the relation between the on-shell and the $\overline{\mathrm{MS}}$ quark mass at three-loop order in QCD. The result has been obtained for the first time with the help of the Padé method [124] and has been confirmed half a year later by a completely independent calculation 126 .

Here, we want to describe the approach of [124] as it constitutes an other facet of applications of the method as described in Section 5.1. In contrast to the examples described before one is directly interested in the real part of the considered function. Furthermore, in the relation between the $\overline{\mathrm{MS}}$ and the on-shell value of the masses we want to know the function $f(z)$ as defined in (5.3) for $z=1$, whereas it is only available for small and large values of $z$. Nevertheless, the method is powerful enough to get the value for $f(1)$ with an error of $2-3 \%$ (see below).

The basic object entering the mass relation is the fermion propagator, $\Sigma(q)$. However, the Padé method cannot be applied directly to $\Sigma(q)$ as it contains (unknown) singularities at threshold. Thus, proper combinations have to be considered which are regular for $z=1$. They are obtained from the requirement that the inverse fermion propagator has a zero at the position of the on-shell mass.

In the following three different types of masses will appear: the bare mass, $m_{0}$, the $\overline{\mathrm{MS}}, m(\mu)$ and the on-shell mass $M$. The relation between them is given by

$$
m(\mu)=Z_{m} m^{0}=z_{m}(\mu) M,
$$

where $z_{m}$ is finite and has an explicit dependence on the renormalization scale $\mu$. It is the purpose of this Section to describe its calculation at order $\alpha_{s}^{3}$.

As already mention above, in order to obtain the mass relation we have to consider the fermion propagator as shown in Eq. (2.15). The renormalized version can be cast in the form

$$
\left(S_{F}(q)\right)^{-1}=i\left[(M-\not q) S_{V}(z)+M\left(z_{m}(\mu) S_{S}(z)-S_{V}(z)\right)\right],
$$

with 27

$$
\begin{aligned}
S_{V}(z) & =Z_{2}\left(1+\Sigma_{V}^{0}\right), \\
S_{S}(z) & =Z_{2} Z_{m}\left(1-\Sigma_{S}^{0}\right) .
\end{aligned}
$$

\footnotetext{
${ }^{27}$ Note that in contrast to the quantities defined in Eq. (5.39) (see also Ref. 177) the wave function renormalization for functions $S_{S / V}$ is still defined in the $\overline{\mathrm{MS}}$ scheme.
} 
$Z_{2}$ denotes the wave function renormalization in the $\overline{\mathrm{MS}}$ scheme which is sufficient for our considerations. Note that the functions $S_{S}$ and $S_{V}$ are $\overline{\mathrm{MS}}$ quantities which later on are expressed in terms of the on-shell mass. The two-loop relation between $m$ and $M$ is enough to do this at order $\alpha_{s}^{3}$. It is convenient to write the functions $S_{S / V}$ in the following way

$$
S_{S / V}=1+\sum_{n \geq 1} S_{S / V}^{(n)}\left(\frac{\alpha_{s}}{\pi}\right)^{n}
$$

where the quantities $S_{S / V}^{(n)}$ exhibit the following colour structures (the indices $S$ and $V$ are omitted in the following):

$$
\begin{aligned}
S^{(1)}= & C_{F} S_{F}, \\
S^{(2)}= & C_{F}^{2} S_{F F}+C_{F} C_{A} S_{F A}+C_{F} T n_{l} S_{F L}+C_{F} T S_{F H}, \\
S^{(3)}= & C_{F}^{3} S_{F F F}+C_{F}^{2} C_{A} S_{F F A}+C_{F} C_{A}^{2} S_{F A A}+C_{F}^{2} T n_{l} S_{F F L}+C_{F}^{2} T S_{F F H} \\
& +C_{F} C_{A} T n_{l} S_{F A L}+C_{F} C_{A} T S_{F A H}+C_{F} T^{2} n_{l}^{2} S_{F L L}+C_{F} T^{2} n_{l} S_{F L H} \\
& +C_{F} T^{2} S_{F H H} .
\end{aligned}
$$

The same decomposition also holds for the function $z_{m}$. In (5.51) $n_{l}$ represents the number of light (massless) quark flavours. $C_{F}$ and $C_{A}$ are the Casimir operators of the fundamental and adjoint representation. In the case of $S U\left(N_{c}\right)$ they are given by $C_{F}=\left(N_{c}^{2}-1\right) /\left(2 N_{c}\right)$ and $C_{A}=N_{c}$. The trace normalization of the fundamental representation is $T=1 / 2$. The subscripts $F, A$ and $L$ in Eq. (5.51) shall remind us on the colour factors $C_{F}, C_{A}$ and $T n_{l}$, respectively. $H$ simply stands for the colour factor $T$.

A formula which allows for the computation of the $\overline{\mathrm{MS}}$-on-shell relation for the quark mass is obtained from the requirement that the inverse fermion propagator has a zero at the position of the on-shell mass:

$$
\left.\left(S_{F}(q)\right)^{-1}\right|_{q^{2}=M^{2}}=0
$$

In the literature there are two different approaches to compute the occuring Feynman diagrams. In the first evaluation of the three-loop $\overline{\mathrm{MS}}-$ on-shell relation the method of Section 5.1 has been applied which we will discuss below. On the contrary, a subsequent analysis [126] has chosen $q^{2}=M^{2}$ from the very beginning which makes it necessary to solve three-loop on-shell integrals. This can effectively be done using the integration-byparts method within dimensional regularization [47]. It enables the derivation of recurrence relations which express complicated integrals in terms of simpler ones. At the end one arrives at a small set of integrals - so-called master integrals - which actually have to be evaluated. In [126, 182 the considerations of [183] have been extended and the missing master integrals have been evaluated. The technique used for the computation is based on the hard-mass procedure for large $M$ which represents the on-shell integrals in terms of a power series in $q^{2} / M^{2}$. The coefficients contain nested harmonic sums which in the on-shell limit, i.e. for $q^{2}=M^{2}$, can be reduced to known mathematical constants. For 
explicit examples we refer to [182]. At the end of this section we will list the analytical result for $z_{m}(\mu)$ obtained in 126 .

The starting point for the approach of [124, 125] is Eq. (5.52). Applying it to Eq. (5.48) leads to the condition

$$
h(z) \equiv z_{m}(\mu) S_{S}(z)-S_{V}(z)=0 \quad \text { for } \quad z=1 .
$$

At a given loop-order $L$, Eqs. (5.49) are inserted and the resulting equation is solved for $z_{m}^{(L)}$. Thus Eq. (5.53) can be cast in the form

$$
h(z)=f(z)+z_{m}^{(L)}\left(\frac{\alpha_{s}}{\pi}\right)^{L} .
$$

Our aim is the computation of $f(1)$. Note that the individual self energies $\Sigma_{S}$ and $\Sigma_{V}$ develop infra-red singularities when they are evaluated on-shell. The proper combination which leads to the relation between the $\overline{\mathrm{MS}}$ and on-shell mass is, however, free of infra-red problems.

The explained procedure has been applied to each colour structure occuring in $f(z)$ separately as outlined in [125]. E.g., collecting all terms proportional to $C_{F}^{3}$ in Eq. (5.54) leads to

$$
f_{F F F}(z)=S_{S, F F F}(z)-S_{V, F F F}(z)+z_{m}^{F} S_{S, F F}+z_{m}^{F F} S_{S, F},
$$

which has to be evaluated for $z=1$. The individual terms on the right-hand side develop a (unknown) singular behaviour which is encoded in the corresponding moments. In case the Padé method is (naively) perfomed with the individual pieces, the Padé approximants try to imitate the threshold singularity. However, due to the very construction of the method the analytical structure of the Padé result is polynomial for $z \rightarrow 1$ and the typical threshold logarithms can not be reproduced. Thus, the results show instabilities in the vicinity of $z=1$. On the contrary, the proper combination as given in Eq. (5.55) has to be regular for $z \rightarrow 1$, as the on-shell mass does not contain any infra-red singularity 184, 185]. The corresponding Padé results demonstrate great stability.

To summarize, although superficially only information about small and large momenta enter the Padé procedure, it is sensitive to the analytical structure at threshold as the information about the singularity is, to some extend, also contained in the moments of the analytical function $f(z)$.

At this place we will refrain from the discussion of the individual colour structures which can be found in [125] but only present the results for the sum where the numerical values for $C_{A}, C_{F}, T$ and $n_{l}$ have been inserted. Still care has to be taken because of the diagrams involving a closed heavy quark loop.

It was already realized in [134 that the Padé procedure shows less stability as soon as diagrams are involved which exhibit more than one particle threshold. In our case the interest is in the lowest particle cut which happens to be for $q^{2}=M^{2}$. The Padé method heavily relies on the combination of expansions in the small and large momentum region. The large momentum expansion, however, is essentially sensitive to the highest particle 


\begin{tabular}{|r|l||r|r|r|r|r|r|}
\hline$n$ & P.A. & $n_{l}=0$ & $n_{l}=1$ & $n_{l}=2$ & $n_{l}=3$ & $n_{l}=4$ & $n_{l}=5$ \\
\hline 5 & {$[4 / 5]$} & -200.2787 & -173.6663 & -148.3787 & -124.4156 & -101.7771 & -80.4628 \\
5 & {$[4 / 6]$} & -201.6419 & -174.8844 & -149.4553 & -125.3553 & -102.5862 & -81.1625 \\
5 & {$[5 / 4]$} & -203.9394 & -176.7290 & -150.8970 & -126.4411 & -103.3591 & -81.6482 \\
5 & {$[5 / 5]$} & -201.4721 & -174.7445 & -149.3422 & -125.2644 & -102.5104 & -81.0786 \\
5 & {$[5 / 6]$} & -198.7799 & -172.8884 & -148.2001 & - & -102.2739 & -81.0336 \\
5 & {$[6 / 4]$} & -202.8435 & -175.8651 & -150.2298 & -125.9387 & -102.9929 & -81.3939 \\
\hline 6 & {$[4 / 6]$} & -201.0906 & -174.3880 & -149.0165 & -124.9749 & -102.2619 & -80.8758 \\
6 & {$[5 / 5]$} & -200.9265 & -174.2458 & -148.8927 & -124.8668 & -102.1673 & -80.7929 \\
6 & {$[5 / 6]$} & -200.4927 & -173.9600 & -148.7433 & -124.8358 & -102.2290 & -80.9131 \\
6 & {$[5 / 7]$} & -200.3603 & -173.7018 & -148.3764 & -124.3940 & -101.7753 & -80.5533 \\
6 & {$[6 / 4]$} & -201.6970 & -174.9293 & -149.4861 & -125.3673 & -102.5725 & -81.1016 \\
6 & {$[6 / 6]$} & -200.3195 & -173.6857 & -148.3751 & -124.3879 & -101.7244 & -80.3848 \\
6 & {$[7 / 5]$} & -202.1300 & -175.2569 & -149.7173 & -125.5125 & -102.6443 & -81.1143 \\
\hline
\end{tabular}

Table 5.5: Padé results for the sum of those contributions which don't have a closed heavy fermion loop. $n_{l}$ has been varied from 0 to 5 .

threshold. Thus, if this threshold numerically dominates the lower-lying ones it cannot be expected that the Padé approximation leads to stable results. In such cases a promising alternative to the above method is the one where only the expansion terms for $q^{2} \rightarrow 0$ are taken into account in order to obtain a numerical value at $q^{2}=M^{2}$. This significantly reduces the calculational effort as the construction of the Padé approximation from lowenergy moments alone is much simpler. In practice this approach will be applied if the Padé results involving also the high-energy data look ill-behaved.

In the present analysis diagrams with other cuts than for $q^{2}=M^{2}$ are already present at the two-loop level (see Fig. 2.2) which allows us to test these ideas. Indeed, taking into account terms up to order $z^{5}$ and performing a Padé approximation there is an agreement of four digits with the exact result [125]. Also at three-loop order either $q^{2}=M^{2}$ or $q^{2}=9 M^{2}$ cuts appear. Cuts involving five or more fermion lines are first possible starting from four-loop order. Note that cuts involving an even number of fermions cannot occur.

Concerning the colour structures introduced in Eq. (5.51) we use for the sum of the structures FFF, FFA, FFL, FAA, FAL and FLL both the low- and high-energy moments whereas for the sum of the structures $F F H, F A H, F L H$ and $F H H$ only the expansion for $z \rightarrow 0$ is used. In Tabs. 5.5 and 5.6 the results for different Padé approximations are listed. $n$ indicates the number of low-energy moments involved in the analysis, i.e. $n=6$ implies the inclusion of terms of $\mathcal{O}\left(z^{6}\right)$. The number of high-energy terms can be obtained in combination with the order of the Padé approximant $([x / y])$ and is given by $x+y+1-n$.

The final result of [124, 125] for the mass relation can be found in Tab. 5.7 where a comparison with the results of [126] is performed. Note that there is perfect agreement for all values of $n_{l}$. At this point we want to mention that the result of 124, 125] is more general as the function $f(z)$ in Eq. (5.54) has been computed for all values of $z$ and not only for the special point $z=1$. This opens the possibility to obtain the fermion 


\begin{tabular}{|r|l||r|r|r|r|r|r|}
\hline$n$ & P.A. & $n_{l}=0$ & $n_{l}=1$ & $n_{l}=2$ & $n_{l}=3$ & $n_{l}=4$ & $n_{l}=5$ \\
\hline 4 & {$[1 / 3]$} & -0.9345 & -0.9572 & -0.9798 & -1.0024 & -1.0249 & -1.0475 \\
4 & {$[2 / 2]$} & -0.9321 & -0.9546 & -0.9770 & -0.9995 & -1.0218 & -1.0442 \\
4 & {$[3 / 1]$} & -0.9324 & -0.9551 & -0.9777 & -1.0003 & -1.0229 & -1.0455 \\
4 & {$[4 / 0]$} & -0.9604 & -0.9828 & -1.0053 & -1.0277 & -1.0501 & -1.0725 \\
\hline 5 & {$[1 / 4]$} & -0.9271 & -0.9495 & -0.9720 & -0.9944 & -1.0169 & -1.0393 \\
5 & {$[2 / 3]$} & -0.9219 & -0.9440 & -0.9661 & -0.9882 & -1.0103 & -1.0324 \\
5 & {$[3 / 2]$} & -0.9086 & -0.9347 & -0.9591 & -0.9827 & -1.0060 & -1.0290 \\
5 & {$[4 / 1]$} & -0.9254 & -0.9478 & -0.9703 & -0.9927 & -1.0151 & -1.0375 \\
5 & {$[5 / 0]$} & -0.9495 & -0.9719 & -0.9942 & -1.0166 & -1.0389 & -1.0613 \\
\hline 6 & {$[1 / 5]$} & -0.9217 & -0.9441 & -0.9665 & -0.9888 & -1.0112 & -1.0336 \\
6 & {$[2 / 4]$} & -0.9140 & -0.9364 & -0.9589 & -0.9813 & -1.0037 & -1.0261 \\
6 & {$[3 / 3]$} & -0.9125 & -0.9352 & -0.9578 & -0.9803 & -1.0028 & -1.0252 \\
6 & {$[4 / 2]$} & -0.9126 & -0.9352 & -0.9578 & -0.9803 & -1.0028 & -1.0253 \\
6 & {$[5 / 1]$} & -0.9202 & -0.9425 & -0.9649 & -0.9872 & -1.0096 & -1.0319 \\
6 & {$[6 / 0]$} & -0.9416 & -0.9639 & -0.9862 & -1.0085 & -1.0308 & -1.0532 \\
\hline
\end{tabular}

Table 5.6: Padé approximations performed in the variable $z$. No high-energy results have been used. Again $n_{l}$ has been varied from 0 to 5 , the dependence, however, is very weak.

\begin{tabular}{|c|c|c|c|c|c|c|c|c|c|}
\hline & \multicolumn{3}{|c|}{$z_{m}(M)=m(M) / M$} & \multicolumn{3}{|c|}{$z_{m}^{S T}(M)=4 m / M$} & \multicolumn{3}{|c|}{$z_{m}^{z n v}(m)=M / \mu_{m}$} \\
\hline$n_{l}$ & $\mathcal{O}\left(\alpha_{s}^{2}\right)$ & $\mathcal{O}\left(\alpha_{s}^{3}\right)|124|$ & $\mathcal{O}\left(\alpha_{s}^{3}\right) \mid 126$ & $\mathcal{\mathcal { O }}\left(\alpha_{s}^{2}\right)$ & $\mathcal{O}\left(\alpha_{s}^{3}\right)$ & $\mathcal{O}\left(\alpha_{s}^{3}\right) \| 126$ & $\mathcal{O}\left(\alpha_{s}^{2}\right)$ & $\mathcal{O \mathcal { O } ( \alpha _ { s } ^ { 3 } )}|124|$ & $\mathcal{O}\left(\alpha_{s}^{3}\right)[126]$ \\
\hline 0 & -14.33 & $-202(5)$ & -198.7 & -11.67 & $-170(5)$ & -166.3 & 13.44 & $194(5)$ & 190.6 \\
\hline 1 & -13.29 & $-176(4)$ & -172.4 & -10.62 & $-146(4)$ & -142.5 & 12.40 & $168(4)$ & 164.6 \\
\hline 2 & -12.25 & $-150(3)$ & -147.5 & -9.58 & $-123(3)$ & -120.0 & 11.36 & $143(3)$ & 139.9 \\
\hline 3 & -11.21 & $-126(3)$ & -123.8 & -8.54 & $-101(3)$ & -98.76 & 10.32 & $119(3)$ & 116.5 \\
\hline 4 & -10.17 & $-103(2)$ & -101.5 & -7.50 & $-81(2)$ & -78.86 & 9.28 & $96(2)$ & 94.42 \\
\hline 5 & -9.13 & $-82(2)$ & -80.40 & -6.46 & $-62(2)$ & -60.27 & 8.24 & $75(2)$ & 73.64 \\
\hline
\end{tabular}

Table 5.7: Two- and three-loop coefficients of the relation between on-shell and $\overline{\mathrm{MS}}$ mass. The choice $\mu^{2}=M^{2}$, respectively, $\mu^{2}=m^{2}$ has been adopted.

propagator in QCD at three-loop order for arbitrary external momentum extending the considerations of [186] by one more loop.

For the relation between the $\overline{\mathrm{MS}}$ and on-shell quark mass one finds up to three loops [32, 123, 124, 125, 126]

$$
\begin{aligned}
\frac{m(\mu)}{M}= & +\frac{\alpha_{s}^{\left(n_{f}\right)}(\mu)}{\pi}\left[-\frac{4}{3}-l_{\mu M}\right]+\left(\frac{\alpha_{s}^{\left(n_{f}\right)}(\mu)}{\pi}\right)^{2}\left[-\frac{3019}{288}-2 \zeta_{2}-\frac{2}{3} \zeta_{2} \ln 2+\frac{1}{6} \zeta_{3}\right. \\
& \left.-\frac{445}{72} l_{\mu M}-\frac{19}{24} l_{\mu M}^{2}+\left(\frac{71}{144}+\frac{1}{3} \zeta_{2}+\frac{13}{36} l_{\mu M}+\frac{1}{12} l_{\mu M}^{2}\right) n_{l}-\frac{4}{3} \sum_{1 \leq i \leq n_{l}} \Delta\left(\frac{M_{i}}{M}\right)\right] \\
& +\left(\frac{\alpha_{s}^{\left(n_{f}\right)}(\mu)}{\pi}\right)^{3}\left[z_{m}^{(3)}(M)+\left(-\frac{165635}{2592}-\frac{25}{3} \zeta_{2}-\frac{25}{9} \zeta_{2} \ln 2+\frac{55}{36} \zeta_{3}\right) l_{\mu M}\right. \\
& -\frac{11779}{864} l_{\mu M}^{2}-\frac{475}{432} l_{\mu M}^{3}+n_{l}\left(\left(\frac{10051}{1296}+\frac{37}{18} \zeta_{2}+\frac{2}{9} \zeta_{2} \ln 2+\frac{7}{9} \zeta_{3}\right) l_{\mu M}+\frac{911}{432} l_{\mu M}^{2}\right.
\end{aligned}
$$




$$
\left.\left.+\frac{11}{54} l_{\mu M}^{3}\right)+n_{l}^{2}\left(\left(-\frac{89}{648}-\frac{1}{9} \zeta_{2}\right) l_{\mu M}^{2}-\frac{13}{216} l_{\mu M}^{2}-\frac{1}{108} l_{\mu M}^{3}\right)\right],
$$

where $\zeta_{2}=\pi^{2} / 6$ and $l_{\mu M}=\ln \mu^{2} / M^{2}$. The constant $z_{m}^{(3)}(M)$ is given by [126]

$$
\begin{aligned}
z_{m}^{(3)}(M)= & -\frac{9478333}{93312}+\frac{55}{162} \ln ^{4} 2+\left(-\frac{644201}{6480}+\frac{587}{27} \ln 2+\frac{44}{27} \ln ^{2} 2\right) \zeta_{2}-\frac{61}{27} \zeta_{3} \\
& +\frac{3475}{432} \zeta_{4}+\frac{1439}{72} \zeta_{2} \zeta_{3}-\frac{1975}{216} \zeta_{5}+\frac{220}{27} a_{4}+n_{l}\left[\frac{246643}{23328}-\frac{1}{81} \ln ^{4} 2\right. \\
& \left.+\left(\frac{967}{108}+\frac{22}{27} \ln 2-\frac{4}{27} \ln ^{2} 2\right) \zeta_{2}+\frac{241}{72} \zeta_{3}-\frac{305}{108} \zeta_{4}-\frac{8}{27} a_{4}\right] \\
& +n_{l}^{2}\left[-\frac{2353}{23328}-\frac{13}{54} \zeta_{2}-\frac{7}{54} \zeta_{3}\right],
\end{aligned}
$$

where $a_{4}=\mathrm{Li}_{4}(1 / 2) \approx 0.517479$.

The function $\Delta(r)$ in Eq. (5.56) takes into account the effects of secondary light quarks. If $0 \leq r \leq 1$ then the function $\Delta(r)$ may be conveniently approximated as follows [123]

$$
\Delta(r)=\frac{\pi^{2}}{8} r-0.597 r^{2}+0.230 r^{3},
$$

which is accurate to $1 \%$. Up to now there is no calculation available taking into account the complete mass dependence of the light quarks at order $\alpha_{s}^{3}$. The subclass of diagrams containing a one-loop light quark vacuum polarization insertion has been considered in [187], where it was observed that the dominant contribution is provided by the linear mass corrections as at order $\alpha_{s}^{2}$ (cf. Eq. (5.58)).

At this point it is worthwhile to compare the results of [124, 125, 126] with estimations for the $\mathcal{O}\left(\alpha_{s}^{3}\right)$ terms obtained with the help of different optimization procedures. In [62] the fastest apparent convergence (FAC) 188 and the principle of minimal sensitivity (PMS) 189 have been used in order to predict the three-loop coefficient of $M / m(m)$. For $n_{l}=2$ one observes a discrepancy of $9 \%$. It even reduces to below $1 \%$ for $n_{l}=5$, i.e. in the case of the top quark. The results obtained in the large $\beta_{0}$-limit [190], where $\beta_{0}$ is the first coefficient of the QCD $\beta$ function, agree to $2 \%$ for $n_{l}=3$, roughly $8 \%$ for $n_{l}=4$ and $17 \%$ for $n_{l}=5$.

Among the various applications of the order $\alpha_{s}^{3}$ term in the $\overline{\mathrm{MS}}-$ on-shell relation we only want to mention the improvement in the determination of the top quark mass to be measured at a future $e^{+} e^{-}$linear collider. To be specific, let us consider the production of top quarks in $e^{+} e^{-}$collisions. The corresponding physical observables expressed in terms of $M_{t}$ show in general a bad convergence behaviour. In the case of the total cross section, e.g., the next-to-next-to-leading order corrections partly exceed the next-to-leading ones. Furthermore the peak position which is the most striking feature of the total cross section and from which finally the mass value can be extracted depends very much on the number of terms one includes into the analysis. The commonly accepted explanation for this is that the pole mass is sensitive to long-distance effects which result in intrinsic uncertainties of order $\Lambda_{Q C D}$ [191, 192]. In other words, it is not possible to determine the pole mass from the analysis of the cross section at threshold with an accuracy better than $\Lambda_{Q C D}$. 
Several strategies have been proposed to circumvent this problem [193, 194, 195]. They are based on the observation that the same kind of ambiguities also appear in the static quark potential, $V(r)$. In the combination $2 M_{t}+V(r)$, however, the infra-red sensitivity drops out. Thus a definition of a short-distance mass extracted from threshold quantities should be possible. The relation of the new mass parameter to the pole mass is used

in order to re-parameterize the threshold phenomena. On the other hand a relation of the new quark mass to the $\overline{\mathrm{MS}}$ mass must be established as it is commonly used for the parameterization of those quantities which are not related to the threshold. In order to do this consistently the three-loop relation between the $\overline{\mathrm{MS}}$ and the on-shell mass is needed.

In 193 the concept of the so-called potential mass, $m_{t, P S}$, has been introduced. Its relation to the $\overline{\mathrm{MS}}$ mass, $m_{t}\left(m_{t}\right)$, reads

$$
m_{t, P S}(20 \mathrm{GeV})=(165.0+6.7+1.2+0.28) \mathrm{GeV},
$$

where the different terms represent the contributions from order $\alpha_{s}^{0}$ to $\alpha_{s}^{3}$. For the numerical values $m_{t}\left(m_{t}\right)=165.0 \mathrm{GeV}$ and $\alpha_{s}^{(6)}\left(m_{t}\left(m_{t}\right)\right)=0.1085$ have been used. The comparison of Eq. (5.59) with the analogous expansion for $M_{t}$,

$$
M_{t}=(165.0+7.6+1.6+0.51) \mathrm{GeV},
$$

shows that the potential mass can be more accurately related to the $\overline{\mathrm{MS}}$ mass than $M_{t}$.

Further details and more examples can be found in [125].

\section{Acknowledgments}

I would like to thank S. Casalbuoni, K.G. Chetyrkin, R. Harlander and B.A. Kniehl for carefully reading the manuscript and for valuable suggestions and discussions. Furthermore I would like to thank J.H. Kühn and B.A. Kniehl for encouraging me to complete this work.

\section{A Technical remarks}

\section{A.1 Asymptotic expansion}

A promising approach to compute - at least in certain kinematical limits - multi-loop diagrams is based on asymptotic expansion. An asymptotic expansion can be considered as a generalization of a Taylor expansion. In both cases one obtains an expansion in powers of a small quantity. However, in case of the asympotitic expansion the corresponding coefficients are not constant but contain non-analytical functions of the small parameter. As a simple example let us consider the function $f(x)=\operatorname{Li}_{2}(1-x)$ for which the Taylor expansion for $x \rightarrow 0$ does not exist beyond the leading order. Nevertheless there is an asymptotic expansion which reads

$$
f(x)=\frac{\pi^{2}}{6}+x(\ln x-1)+\mathcal{O}\left(x^{2} \ln x\right),
$$


with the non-analytic (for $x \rightarrow 0$ ) function $\ln x$ in the coefficient of the linear term in $x$.

In the case of Feynman diagrams the situation is similar. Systematic procedures have been developed in the case of a large external mometum ("large-momentum procedure") and a large internal mass ("hard-mass procedure") [129]. Both procedures apply to problems which can be formulated in Euclidean space. This is the case for all calculations presented in this review. In contrast to that there are also phenomena which are tightly connected to the Minkowskian space-time. Also in such cases rules for an asymptotic expansion have been developed. In particular, two-loop on-shell two-point diagrams [196] and two-loop vertex diagrams in the Sudakov limit 197 have been considered. Also the threshold expansion 198 belongs to this class of phenomena.

One may treat the large-momentum and hard-mass procedures on the same footing. Thus, in what follows we only present the general formulae in the case of large external momenta - the transition to the hard-mass procedure is straightforward. The prescription for the large-momentum procedure is summarized by the following formulaz:

$$
\Gamma(Q, m, q) \stackrel{Q \rightarrow \infty}{\simeq} \sum_{\gamma} \Gamma / \gamma(q, m) \star T_{\left\{q_{\gamma}, m_{\gamma}\right\}} \gamma\left(Q, m_{\gamma}, q_{\gamma}\right)
$$

Here, $\Gamma$ is the Feynman diagram under consideration, $\{Q\}(\{m, q\})$ is the collection of the large (small) parameters, and the sum goes over all subgraphs $\gamma$ of $\Gamma$ with masses $m_{\gamma}$ and external momenta $q_{\gamma}$, subject to certain conditions to be described below. $T_{\{q, m\}}$ is an operator performing a Taylor expansion in $\{q, m\}$ before any integration is carried out. The notation $\Gamma / \gamma \star T_{\{q, m\}} \gamma$ indicates that the subgraph $\gamma$ of $\Gamma$ is replaced by its Taylor expansion which should be performed in all masses and external momenta of $\gamma$ that do not belong to the set $\{Q\}$. In particular, also those external momenta of $\gamma$ that appear to be integration momenta in $\Gamma$ have to be considered as small. Only after the Taylor expansions have been carried out, the loop integrations are performed. In the following we will refer to the set $\{\gamma\}$ as hard subgraphs or simply subgraphs and to $\{\Gamma / \gamma\}$ as co-subgraphs.

The conditions for the subgraphs $\gamma$ are different for the hard-mass and largemomentum procedures 2 . For the large-momentum procedure, $\gamma$ must

- contain all vertices where a large momentum enters or leaves the graph

- be one-particle irreducible after identifying these vertices.

From these requirements it is clear that the hard subgraphs become massless integrals where the scales are given by the large momenta. In the simplest case of one large momentum one ends up with propagator-type integrals. The co-subgraph, on the other hand, may still contain small external momenta and masses. However, the resulting integrals are typically much simpler than the original one.

In the case of hard-mass procedure, $\gamma$ has to

\footnotetext{
${ }^{28}$ In the case of the hard-mass procedure one essentially has to replace $Q$ by the large mass $M$.

${ }^{29}$ Actually they are very similar and it is certainly possible to merge them into one condition using a more abstract language. For our purpose, however, it is more convenient to distinguish the two procedures.
} 


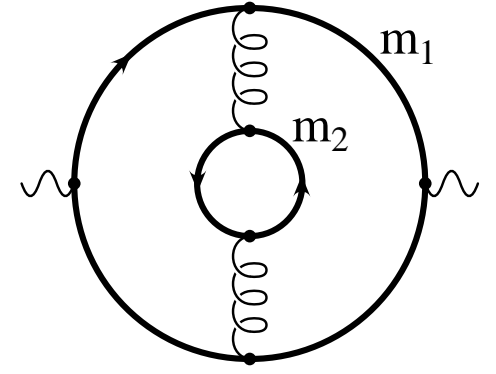

(a)

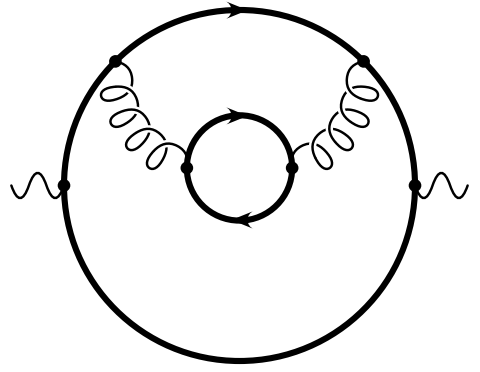

(b)

Figure A.1: Fermionic double-bubble diagrams with generic masses $m_{1}$ and $m_{2}$.

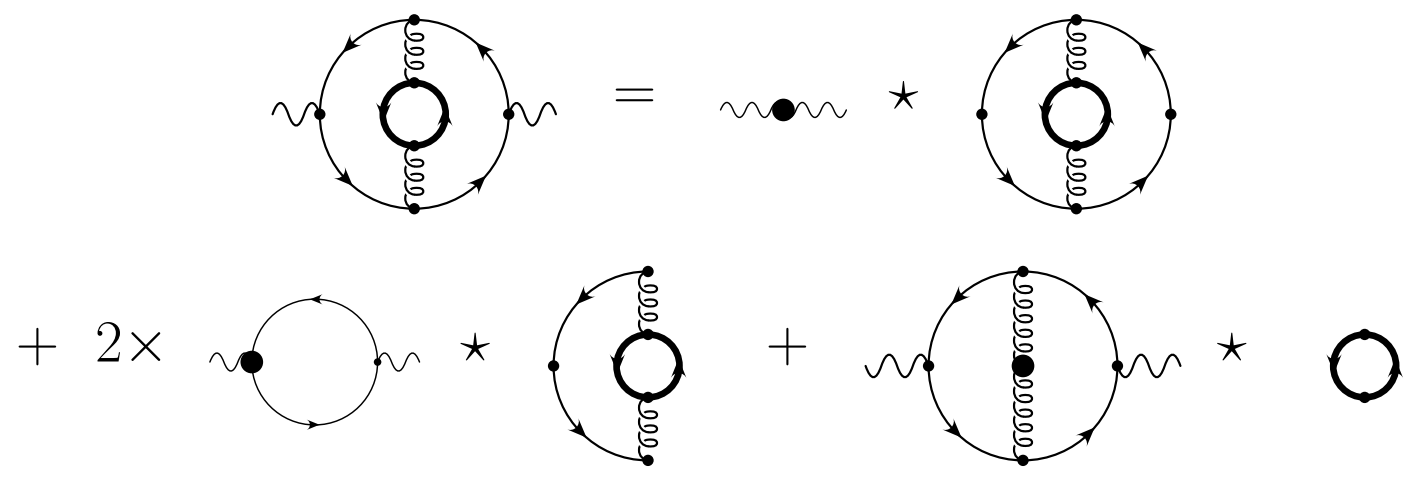

Figure A.2: Hard-mass procedure for the double-bubble diagram. The hierarchy $q^{2} \ll m^{2}$ is considered where $m$ is the mass of the inner line. The hard subdiagrams (right of " $\star$ ") are to be expanded in all external momenta including $q$ and reinserted into the fat vertex dots of the co-subgraphs (left of " $\star$ ").

- contain all the propagators carrying a large mass

- be one-particle irreducible in its connected parts after contracting the heavy lines.

Here, the hard subgraphs reduce to tadpole integrals with the large masses setting the scales. The co-subgraphs are again simpler to evaluate than the initial diagram.

An example demonstrating the practical application of the large-momentum expansion was already presented in Section 4 (cf. Fig. 4.8; see also [116]). As an application of the hard-mass procedure let us consider the double-bubble diagram of Fig. A.1 with the hierarchy $m_{1}^{2} \ll q^{2} \ll m_{2}^{2}$. The imaginary part leads to contributions for the total cross section $\sigma\left(e^{+} e^{-} \rightarrow\right.$ hadrons $)$. One may think of charm quark production $\left(m_{1}=M_{c}\right)$ in the presence of a virtual bottom quark $\left(m_{2}=M_{b}\right)$. It turns out that already the first term provides a very good approximation almost up to the threshold $\sqrt{s}=2 M_{b}$ [199, 200, 201]. For simplicity we set $m_{1}=0$ and $m_{2}=m$ in the following.

The corresponding diagrammatic representation is shown in Fig. A.2. There are three 
subdiagrams, where one again corresponds to the naive Taylor expansion of the integrand in the external momentum $q$. After Taylor expansion, the subdiagrams are reduced to tadpole integrals with mass scale $m$. The scale of the co-subgraphs is given by $q$, thus leading to massless propagator-type integrals. The result for the first three terms reads [202, 116]

$$
\begin{aligned}
\bar{\Pi}_{g s}\left(q^{2}\right) \stackrel{q^{2} \lll m^{2}}{=} & \frac{3}{16 \pi^{2}}\left(\frac{\alpha_{s}}{\pi}\right)^{2} C_{\mathrm{F}} T\left[\frac{295}{648}+\frac{11}{6} l_{q \mu}-\frac{1}{6} l_{q \mu}^{2}-\frac{11}{6} l_{q m}+\frac{1}{6} l_{q m}^{2}\right. \\
& -\frac{4}{3} \zeta_{3} l_{q \mu}+\frac{4}{3} \zeta_{3} l_{q m}+\frac{q^{2}}{m^{2}}\left(\frac{3503}{10125}-\frac{88}{675} l_{q m}+\frac{2}{135} l_{q m}^{2}\right) \\
& \left.+\left(\frac{q^{2}}{m^{2}}\right)^{2}\left(-\frac{2047}{514500}+\frac{1303}{529200} l_{q m}-\frac{1}{2520} l_{q m}^{2}\right)\right]+\cdots
\end{aligned}
$$

with $l_{q \mu}=\ln \left(-q^{2} / \mu^{2}\right)$ and $l_{q m}=\ln \left(-q^{2} / m^{2}\right)$.

We want to stress that the main simplification, which is common to all kinds of asymtotic expansions, comes from the fact that the expansions in the small parameters are done before any momentum integration is performed. The proof that this leads to correct results is based on the so-called strategy of regions [203]. There different regions of each loop momentum are selected and in each of them Taylor expansions with respect to the small parameters are performed. In the limits of the hard-mass and large-momentum procedures an interpretation of the different regions in terms subgraphs and cosub-graphs is possible (see above). This is different in the case of the threshold expansion 198 where a graphical representation becomes much less transparent. However, the application of the strategy of regions [203] leads to correct results.

\section{A.2 Single-scale Feynman diagrams up to three loops}

The problem of evaluating one-loop Feynman diagrams is - at least in principle - solved (see, e.g., Refs. [3], 7, 204, 9]). However, in case many legs and lots of different masses appear also one-loop computations can become very tedious, in particular if degenerate momentum configurations are involved.

At two-loop order the class of Feynman diagrams which have been studied in detail is much more restricted. There is a good understanding of two-point functions (see, e.g., [10]) which also has found important physical applications [205]. Concerning threeand four-point functions one is essentially restricted to the massless case. In this context we want to draw the attention to the recent activity in the computation of the two-loop box diagrams (for a brief overview see [11]). Within the last two years the basis has been established to compute two-loop virtual corrections to the four-point Feynman amplitudes where all internal lines are massless and at most one external leg is off-shell. This opens the door to investigate next-to-next-to-leading order processes like the two-jet production at hadron colliders or three-jet production in $e^{+} e^{-}$annihilation.

It is obvious that the complexity of the computation of a Feynman diagram strongly depends on the number of different scales involved. There is one class of diagrams which is studied in great detail up to three loops, namely diagrams which only depend on 
one dimensionful scale. Next to massless propagator-type diagrams with one external momentum, $q$, we have in mind vacuum integrals with one non-zero mass, $M$ and socalled on-shell integrals for which the condition $q^{2}=M^{2}$ is fulfilled.

The basic idea for the computation of the integrals is common to all three types: after the numerator is decomposed in terms of the denominator recurrence relations are applied which express the diagram as a linear combination of so-called master integrals. Only for the latter a hard computation is necessary. However, since in the case of single-scale diagrams the master integrals are essentially pure numbers it is also possible to use highprecision numerical methods in case an analytical calculation is not possible. We want to mention that for the massless propagator-type and the massive vacuum integrals two and nine master integrals are needed, respectively. In the case of the three-loop on-shell integrals a list of all master integrals can be found in the Appendix of Ref. [182]. Counting also those integrals which are composed of products of lower-order diagrams they amount to 18.

For convenience we want to provide the analytical results for the massless one-loop two-point functions, $P_{a b}(Q)$, the on-shell two-point functions, $O_{a b}(Q)$, and the one- $\left(V_{a}\right)$ and two-loop $\left(V_{a b c}\right)$ vacuum integrals in Euclidian space.

$$
\begin{aligned}
P_{a b}(Q) & =\int \frac{\mathrm{d}^{D} p}{(2 \pi)^{D}} \frac{1}{p^{2 a}(p+Q)^{2 b}} \\
& =\frac{\left(Q^{2}\right)^{D / 2-a-b}}{(4 \pi)^{D / 2}} \frac{\Gamma(a+b-D / 2) \Gamma(D / 2-a) \Gamma(D / 2-b)}{\Gamma(a) \Gamma(b) \Gamma(D-a-b)} \\
O_{a b}(Q) & =\int \frac{\mathrm{d}^{D} p}{(2 \pi)^{D}} \frac{1}{p^{2 a}\left(p^{2}+2 p \cdot Q\right)^{b}} \\
& =\frac{\left(Q^{2}\right)^{D / 2-a-b}}{(4 \pi)^{D / 2}} \frac{\Gamma(a+b-D / 2) \Gamma(D-2 a-b)}{\Gamma(b) \Gamma(D-a-b)}, \\
V_{a} & =\int \frac{\mathrm{d}^{D} p}{(2 \pi)^{D}} \frac{1}{\left(p^{2}+M^{2}\right)^{a}}=\frac{\left(M^{2}\right)^{D / 2-a}}{(4 \pi)^{D / 2}} \frac{\Gamma(a-D / 2)}{\Gamma(a)} \\
V_{a b c} & =\int \frac{\mathrm{d}^{D} p}{(2 \pi)^{D}} \frac{\mathrm{d}^{D} k}{(2 \pi)^{D}} \frac{1}{\left(p^{2}+M^{2}\right)^{a}\left(k^{2}+M^{2}\right)^{b}\left((p+k)^{2}\right)^{c}} \\
& =\frac{\left(M^{2}\right)^{D-a-b-c} \Gamma(a+b+c-D) \Gamma(a+c-D / 2) \Gamma(b+c-D / 2) \Gamma(D / 2-c)}{(4 \pi)^{D}} \frac{\Gamma(a) \Gamma(b) \Gamma(a+b+2 c-D) \Gamma(D / 2)}{}
\end{aligned}
$$

The results of a general three-loop diagram can not be expressed in terms of $\Gamma$ functions. Moreover, due to the large number of contributing diagrams and the complexity of intermediate expressions it is absolutely necessary to use computer algebra programs for the computation of multi-loop diagrams. It is thus also hardly possible to provide intermediate results which eventually could be used in other calculations. Therefore, on one side one is left with the description of the method used for the evaluation of the dia- 
grams. On the other hand it is possible to provide the program code which was used for the computation. Thus everybody can repeat the calculation or apply it to own problems.

Both for the massless propagator-type integrals and the massive vacuum integrals FORM [55 packages have been published: massless integrals up to three loops can be computed using MINCER [31]; the package for the massive integrals is called MATAD [35]. In the following we will present an example which demonstrates the use of MATAD. The use of MINCER is very similar. Actually, in the package GEFICOM [41] MATAD and MINCER are used in parallel using the same notation for the input.

Let us consider the triangle diagram as pictured in Fig. A.3. It is one of the 657 diagrams which contribute to the coefficient function $C_{1}^{0}$ appearing in the effective Lagrangian of Eq. (4.13). According to the Lorentz structure the result can be written as

$$
K\left(M_{t}\right)\left(q_{1}^{\nu} q_{2}^{\mu}-q_{1} q_{2} g^{\mu \nu}\right)
$$

where $q_{1}$ and $q_{2}$ are the momenta of the gluons with polarization vectors $\epsilon^{\mu}\left(q_{1}\right)$ and $\epsilon^{\nu}\left(q_{2}\right)$. Thus the vertex diagrams have to be expanded up to linear order both in $q_{1}$ and $q_{2}$, and an appropriate projector has to be applied in order to get $K\left(M_{t}\right)$.

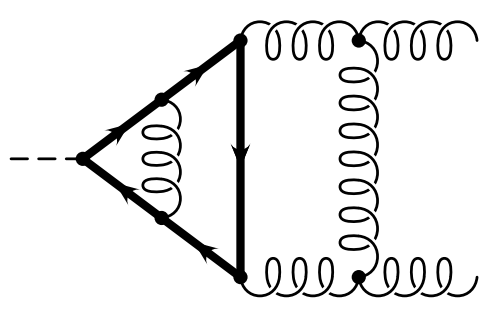

Figure A.3: Sample diagram contributing to the decay of the Higgs boson. Solid and looped lines represent quarks and gluons, respectively.

MATAD requires one file containing the diagrams and the projectors which have to be applied. In our case it could look as follows

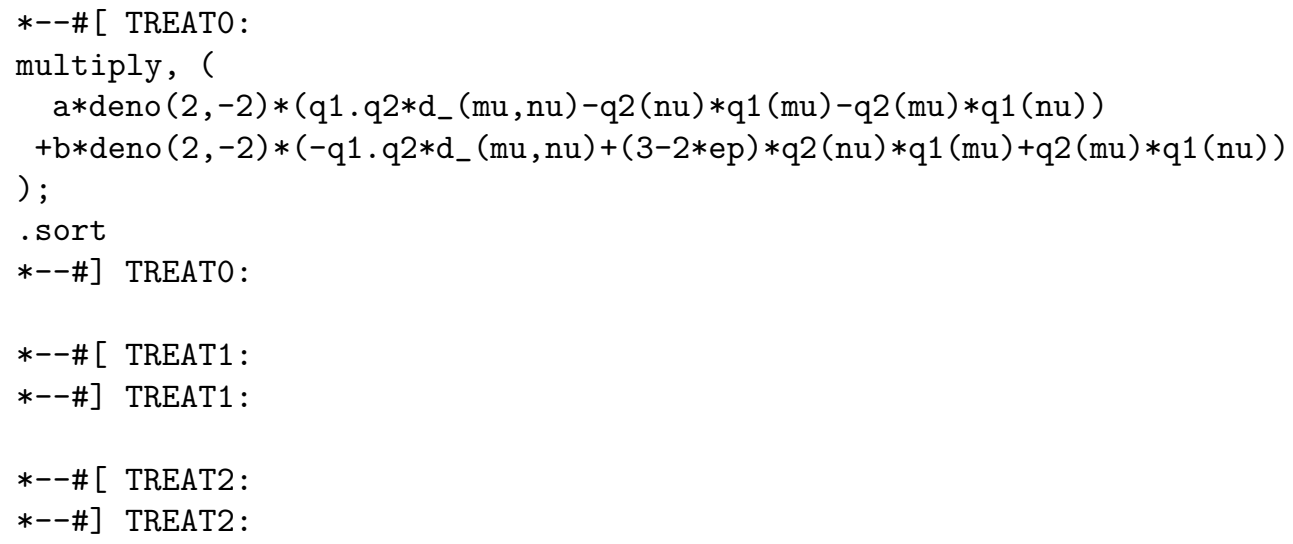




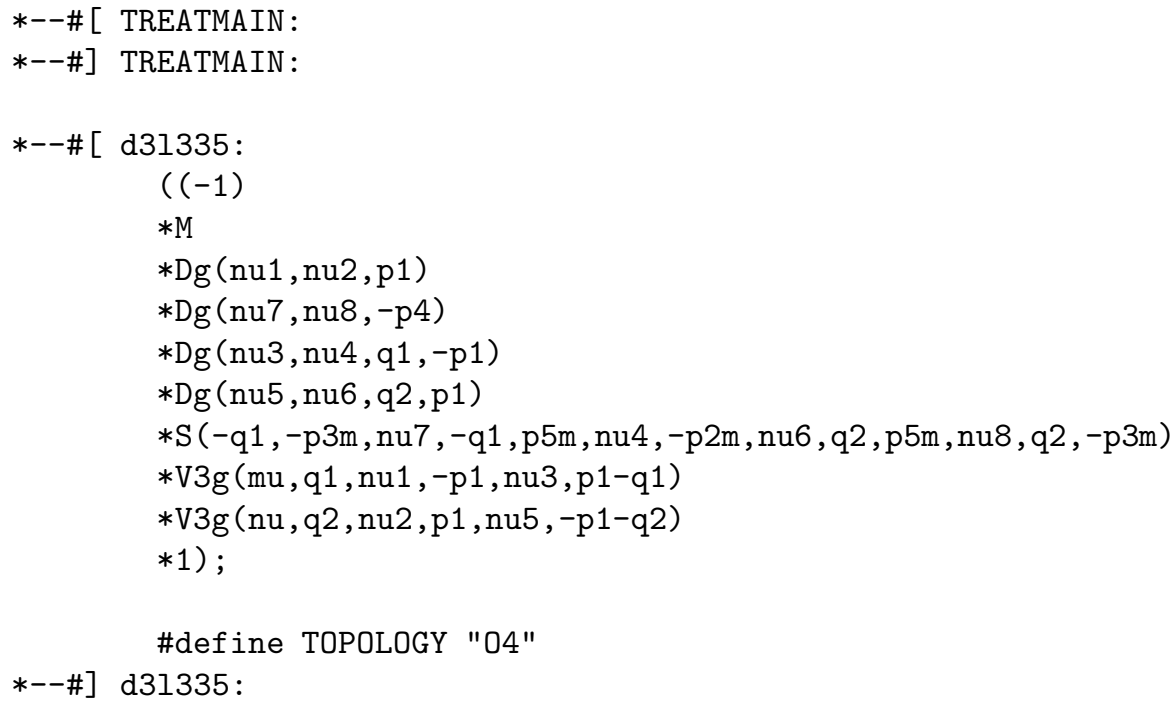

where the diagram d31335 corresponds to the one shown in Fig. A.3 (for details concerning the nomenclature of the momenta see [35]). The fold TREATO contains (up to an overall factor $\left.\left(q_{1} \cdot q_{2}\right)^{-2}\right)$ the projector on the coefficients in front of the structures $g^{\mu \nu}$ and $q_{1}^{\nu} q_{2}^{\mu}$ of Eq. (A.8). They are marked by the symbols a and b, respectively. Thus the transversality of Eq. (A.8) can be explicitly checked in the sum of all contributing diagrams (the result of a single diagram does in general not have a transverse structure).

A second file which is required, the so-called main-file, reads

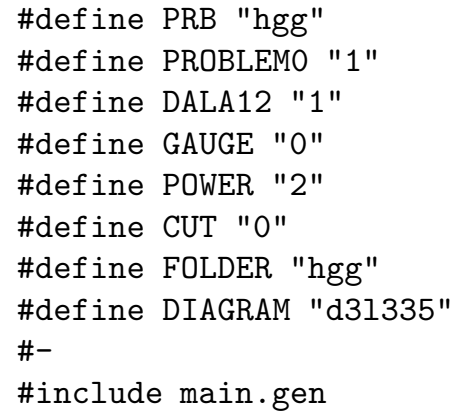

The fifth line ensures that an expansion of the integrand up to the second order in the external momenta is performed and the third line sets $q_{1}^{2}$ and $q_{2}^{2}$ to zero and factors out the scalar product $q_{1} \cdot q_{2}$. \#define CUT "0" sets $\varepsilon$ to zero in the final result. In this example we choose Feynman gauge which is achieved with \#define GAUGE "0".

After calling MATAD it takes of the order of a minute to obtain the result:

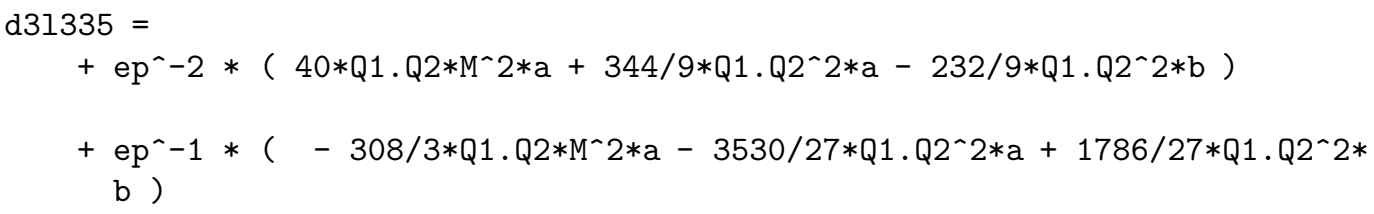




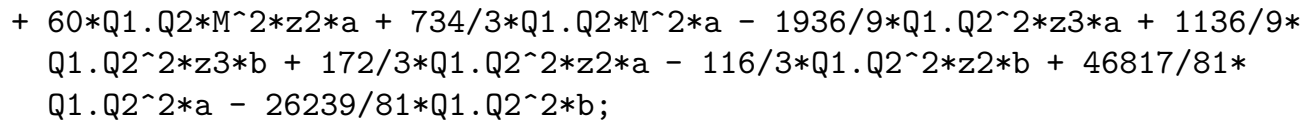

Note that the terms proportional to Q1.Q2* $\mathrm{M}^{\wedge} 2$ cancel after adding all contributing diagrams.

\section{A.3 Automation of Feynman diagram computation}

In this section we briefly want to mention the program packages which have been used to obtain most of the results discussed in this review. For a general overview concerning the automation of Feynman diagram computation we refer to [116].

The large number of diagrams which occurs in particular if one considers higher loop orders makes it necessary to generate the diagrams automatically. The Fortran program QGRAF [57] provides the possiblility to implement own models in a simple way. Furthermore it is quite fast and generates several thousand diagrams in a few seconds. One of the disadvantages of QGRAF is that the user has to put the Feynman rules himself. On the other hand, this provides quite some flexibility in the choices of the vertices. E.g., it is straightforward to implement the vertex involving the coupling of the operator $\mathcal{O}_{1}$ to four gluons (cf. Eq. (4.1)).

In general, the application of asymptotic expansions, in particular if serveral of them are applied successively, generates many subdiagram which have to be expanded in several small quantities. Even for a single multi-loop diagram this becomes very tedious if it has to be performed by hand. For this reason the programs LMP [74] and EXP [180] have been developed. LMP was especially developed in order to apply the large-momentum procedure to the diagonal current correlators 1136, 137, 95, 110. In some sense EXP can be considered as the successor of LMP. Next to the hard-mass procedure also the succesive application of large-mometum and/or hard-mass procedure is possible. This broadens the area of applications. Here we just want to mention as examples the correction of $\mathcal{O}\left(\alpha \alpha_{s}\right)$ to the $Z$ boson decay [206, $\mathcal{O}\left(\alpha_{s}^{2}\right)$ corrections to the top quark decay [177] or two-loop QED corrections to the muon decay [19] (cf. Section 5.3).

The very computation of the integrals is performed with the program packages MINCER [31] and MATAD [35] (see Appendix A.2]). The former deals with massless propagator-type integrals up to three loops and MATAD was written to deal with vacuum diagrams at one-, two- and three-loop order where several of the internal lines may have a common mass. The area of application for each of the individual packages seems to be quite restrictive. However, in particular the combined application offers a quite flexible use.

In order to handle problems where a large number of diagrams are involved and where eventually an asymptotic expansion has to be applied in a convenient way the program package GEFICOM has been written. A very limited number of small input files allows the user to rule the flow of the computation. Qgraf is called to generate the diagrams. A

Mathematica 42] script determines the toplogy of each individual diagram and provides input which either can be directly read from MINCER and/or MATAD or can be passed to EXP 
or LMP. At the end the results of the individual diagrams are summed and the bare result is stored. Moreover a convenient environment is provided which, e.g., makes sure that all result files are up-to-date. Thus, processes involving a large number of (sub-)diagrams can be treated without taking care of each individual result.

\section{B Decoupling constants and coefficient functions}

Transforming the decoupling constants of Eqs. (3.28) and (3.30) to the $\overline{\mathrm{MS}}$ scheme one obtains

$$
\begin{aligned}
\zeta_{m}^{\mathrm{MS}}= & +\left(\frac{\alpha_{s}^{\left(n_{f}\right)}(\mu)}{\pi}\right)^{2}\left(\frac{89}{432}-\frac{5}{36} \ln \frac{\mu^{2}}{m_{h}^{2}}+\frac{1}{12} \ln ^{2} \frac{\mu^{2}}{m_{h}^{2}}\right)+\left(\frac{\alpha_{s}^{\left(n_{f}\right)}(\mu)}{\pi}\right)^{3}\left[\frac{2951}{2916}\right. \\
& -\frac{407}{864} \zeta_{3}+\frac{5}{4} \zeta_{4}-\frac{1}{36} B_{4}+\left(-\frac{311}{2592}-\frac{5}{6} \zeta_{3}\right) \ln \frac{\mu^{2}}{m_{h}^{2}}+\frac{175}{432} \ln ^{2} \frac{\mu^{2}}{m_{h}^{2}} \\
& \left.+\frac{29}{216} \ln ^{3} \frac{\mu^{2}}{m_{h}^{2}}+n_{l}\left(\frac{1327}{11664}-\frac{2}{27} \zeta_{3}-\frac{53}{432} \ln \frac{\mu^{2}}{m_{h}^{2}}-\frac{1}{108} \ln ^{3} \frac{\mu^{2}}{m_{h}^{2}}\right)\right] \\
\approx & 1+0.2060\left(\frac{\alpha_{s}^{\left(n_{f}\right)}\left(\mu_{h}\right)}{\pi}\right)^{2}+\left(1.8476+0.0247 n_{l}\right)\left(\frac{\alpha_{s}^{\left(n_{f}\right)}\left(\mu_{h}\right)}{\pi}\right)^{3}, \\
\left(\zeta_{g}^{\mathrm{MS}}\right)^{2}= & 1+\frac{\alpha_{s}^{\left(n_{f}\right)}(\mu)}{\pi}\left(-\frac{1}{6} \ln \frac{\mu^{2}}{m_{h}^{2}}\right)+\left(\frac{\alpha_{s}^{\left(n_{f}\right)}(\mu)}{\pi}\right)^{2}\left(\frac{11}{72}-\frac{11}{24} \ln \frac{\mu^{2}}{m_{h}^{2}}+\frac{1}{36} \ln ^{2} \frac{\mu^{2}}{m_{h}^{2}}\right) \\
& +\left(\frac{\alpha_{s}^{\left(n_{f}\right)}(\mu)}{\pi}\right)^{3}\left[\frac{564731}{124416}-\frac{82043}{27648} \zeta_{3}-\frac{955}{576} \ln \frac{\mu^{2}}{m_{h}^{2}}+\frac{53}{576} \ln ^{2} \frac{\mu^{2}}{m_{h}^{2}}\right. \\
& \left.-\frac{1}{216} \ln ^{3} \frac{\mu^{2}}{m_{h}^{2}}+n_{l}\left(-\frac{2633}{31104}+\frac{67}{576} \ln \frac{\mu^{2}}{m_{h}^{2}}-\frac{1}{36} \ln ^{2} \frac{\mu^{2}}{m_{h}^{2}}\right)\right] \\
\approx & 1+0.1528\left(\frac{\alpha_{s}^{\left(n_{f}\right)}\left(\mu_{h}\right)}{\pi}\right)^{2}+\left(0.9721-0.0847 n_{l}\right)\left(\frac{\alpha_{s}^{\left(n_{f}\right)}\left(\mu_{h}\right)}{\pi}\right)^{3} .
\end{aligned}
$$

In the following, we list the decoupling constants $\zeta_{m}$ and $\zeta_{g}$ appropriate for the general gauge group $\mathrm{SU}\left(N_{c}\right)$. The results read

$$
\begin{aligned}
\zeta_{m}^{\mathrm{MS}}= & 1+\left(\frac{\alpha_{s}^{\left(n_{f}\right)}(\mu)}{\pi}\right)^{2}\left(\frac{1}{N_{c}}-N_{c}\right)\left(-\frac{89}{1152}+\frac{5}{96} \ln \frac{\mu^{2}}{m_{h}^{2}}-\frac{1}{32} \ln ^{2} \frac{\mu^{2}}{m_{h}^{2}}\right) \\
& +\left(\frac{\alpha_{s}^{\left(n_{f}\right)}(\mu)}{\pi}\right)^{3}\left\{\frac{1}{N_{c}^{2}}\left(-\frac{683}{4608}+\frac{57}{256} \zeta_{3}-\frac{9}{64} \zeta_{4}+\frac{1}{32} B_{4}\right)\right. \\
& +\frac{1}{N_{c}}\left(\frac{1685}{62208}-\frac{7}{144} \zeta_{3}\right)+\frac{907}{31104}-\frac{397}{2304} \zeta_{3}-\frac{1}{32} B_{4}
\end{aligned}
$$




$$
\begin{aligned}
& +N_{c}\left(-\frac{1685}{62208}+\frac{7}{144} \zeta_{3}\right)+N_{c}^{2}\left(\frac{14813}{124416}-\frac{29}{576} \zeta_{3}+\frac{9}{64} \zeta_{4}\right) \\
& +\left[\frac{1}{N_{c}^{2}}\left(-\frac{13}{512}+\frac{3}{32} \zeta_{3}\right)+\frac{31}{864 N_{c}}+\frac{1}{32}-\frac{31}{864} N_{c}-N_{c}^{2}\left(\frac{3}{512}\right.\right. \\
& \left.\left.+\frac{3}{32} \zeta_{3}\right)\right] \ln \frac{\mu^{2}}{m_{h}^{2}}+\left(-\frac{1}{32 N_{c}^{2}}-\frac{5}{576 N_{c}}-\frac{5}{384}+\frac{5}{576} N_{c}+\frac{17}{384} N_{c}^{2}\right) \ln ^{2} \frac{\mu^{2}}{m_{h}^{2}} \\
& +\left(\frac{1}{144 N_{c}}-\frac{11}{576}-\frac{1}{144} N_{c}+\frac{11}{576} N_{c}^{2}\right) \ln ^{3} \frac{\mu^{2}}{m_{h}^{2}} \\
& \left.+n_{l}\left(\frac{1}{N_{c}}-N_{c}\right)\left(-\frac{1327}{31104}+\frac{1}{36} \zeta_{3}+\frac{53}{1152} \ln \frac{\mu^{2}}{m_{h}^{2}}+\frac{1}{288} \ln ^{3} \frac{\mu^{2}}{m_{h}^{2}}\right)\right\}, \\
& \left(\zeta_{g}^{\mathrm{MS}}\right)^{2}=1+\frac{\alpha_{s}^{\left(n_{f}\right)}(\mu)}{\pi}\left(-\frac{1}{6} \ln \frac{\mu^{2}}{m_{h}^{2}}\right) \\
& +\left(\frac{\alpha_{s}^{\left(n_{f}\right)}(\mu)}{\pi}\right)^{2}\left[\frac{13}{192 N_{c}}+\frac{25}{576} N_{c}-\left(\frac{1}{16 N_{c}}+\frac{7}{48} N_{c}\right) \ln \frac{\mu^{2}}{m_{h}^{2}}+\frac{1}{36} \ln ^{2} \frac{\mu^{2}}{m_{h}^{2}}\right] \\
& +\left(\frac{\alpha_{s}^{\left(n_{f}\right)}(\mu)}{\pi}\right)^{3}\left\{\frac{1}{N_{c}^{2}}\left(-\frac{97}{2304}+\frac{95}{1536} \zeta_{3}\right)+\frac{1}{N_{c}}\left(-\frac{103}{10368}+\frac{7}{512} \zeta_{3}\right)\right. \\
& -\frac{1063}{5184}+\frac{893}{3072} \zeta_{3}+N_{c}\left(\frac{451}{20736}-\frac{7}{256} \zeta_{3}\right)+N_{c}^{2}\left(\frac{7199}{13824}-\frac{17}{48} \zeta_{3}\right) \\
& +\left(-\frac{9}{256 N_{c}^{2}}-\frac{5}{192 N_{c}}-\frac{119}{1152}-\frac{23}{3456} N_{c}-\frac{1169}{6912} N_{c}^{2}\right) \ln \frac{\mu^{2}}{m_{h}^{2}} \\
& +\left(\frac{5}{192 N_{c}}-\frac{11}{384}+\frac{35}{576} N_{c}-\frac{1}{128} N_{c}^{2}\right) \ln ^{2} \frac{\mu^{2}}{m_{h}^{2}}-\frac{1}{216} \ln ^{3} \frac{\mu^{2}}{m_{h}^{2}} \\
& +n_{l}\left[\frac{41}{1296 N_{c}}-\frac{329}{10368} N_{c}+\left(-\frac{5}{384 N_{c}}+\frac{139}{3456} N_{c}\right) \ln \frac{\mu^{2}}{m_{h}^{2}}\right. \\
& \left.\left.+\left(\frac{1}{96 N_{c}}-\frac{1}{96} N_{c}\right) \ln ^{2} \frac{\mu^{2}}{m_{h}^{2}}\right]\right\} \text {. }
\end{aligned}
$$

For $N_{c}=3$, we recover Eqs. (B.1) and (B.2).

The renormalized decoupling constants $\zeta_{2}$ and $\zeta_{3}$ for the quark and gluon fields, respectively, arise from Eqs. (3.15) and (3.10). Of course, $\zeta_{2}$ and $\zeta_{3}$ are both gauge dependent. Restricting ourselves to the case $N_{c}=3$, we find in the covariant gauge (3.2)

$$
\begin{aligned}
\zeta_{2}^{\mathrm{MS}}= & +\left(\frac{\alpha_{s}^{\left(n_{f}\right)}(\mu)}{\pi}\right)^{2}\left(\frac{5}{144}-\frac{1}{12} \ln \frac{\mu^{2}}{m_{h}^{2}}\right)+\left(\frac{\alpha_{s}^{\left(n_{f}\right)}(\mu)}{\pi}\right)^{3}\left[\frac{42811}{62208}+\frac{1}{18} \zeta_{3}\right. \\
& -\frac{155}{192} \ln \frac{\mu^{2}}{m_{h}^{2}}+\frac{49}{576} \ln ^{2} \frac{\mu^{2}}{m_{h}^{2}}-\frac{1}{96} \ln ^{3} \frac{\mu^{2}}{m_{h}^{2}}+n_{l}\left(\frac{35}{3888}+\frac{5}{432} \ln \frac{\mu^{2}}{m_{h}^{2}}\right)
\end{aligned}
$$




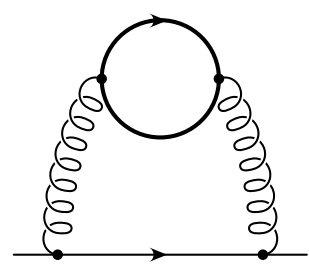

(a)

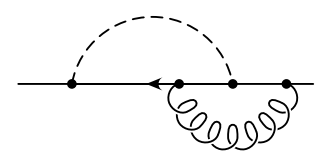

(e)

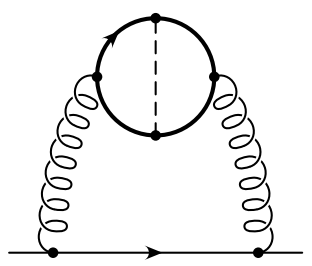

(b)

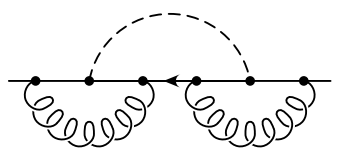

(f)

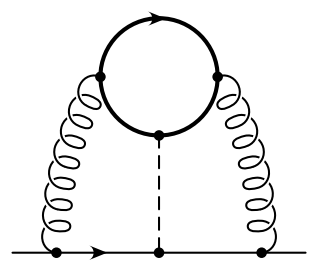

(c)

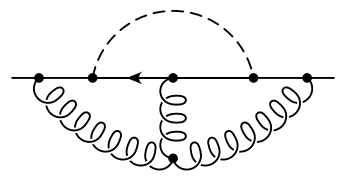

(g)

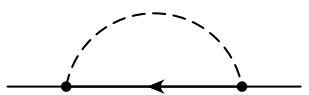

(d)

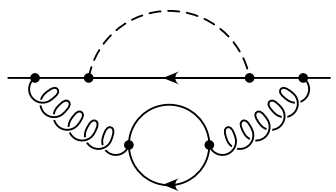

(h)

Figure B.1: Feynman diagrams contribution to $Z_{2}, Z_{m}$ and $\zeta_{m_{q}}^{0}$. The dashed line either represents the Higgs boson $(h)$ or the neutral $(\chi)$ or charged $\left(\phi^{ \pm}\right)$Goldstone boson.

$$
\begin{aligned}
& \left.+\xi\left(-\frac{2387}{6912}+\frac{1}{12} \zeta_{3}+\frac{121}{576} \ln \frac{\mu^{2}}{m_{h}^{2}}-\frac{13}{192} \ln ^{2} \frac{\mu^{2}}{m_{h}^{2}}+\frac{1}{96} \ln ^{3} \frac{\mu^{2}}{m_{h}^{2}}\right)\right] \\
\zeta_{3}^{\mathrm{MS}}= & +\frac{\alpha_{s}^{\left(n_{f}\right)}(\mu)}{\pi}\left(\frac{1}{6} \ln \frac{\mu^{2}}{m_{h}^{2}}\right)+\left(\frac{\alpha_{s}^{\left(n_{f}\right)}(\mu)}{\pi}\right)^{2}\left(\frac{91}{1152}+\frac{29}{96} \ln \frac{\mu^{2}}{m_{h}^{2}}+\frac{3}{32} \ln ^{2} \frac{\mu^{2}}{m_{h}^{2}}\right) \\
& +\left(\frac{\alpha_{s}^{\left(n_{f}\right)}(\mu)}{\pi}\right)^{3}\left[-\frac{284023}{62208}+\frac{86183}{27648} \zeta_{3}+\frac{99}{128} \zeta_{4}-\frac{1}{32} B_{4}\right. \\
& +\left(\frac{52433}{27648}-\frac{33}{64} \zeta_{3}\right) \ln \frac{\mu^{2}}{m_{h}^{2}}+\frac{383}{2304} \ln ^{2} \frac{\mu^{2}}{m_{h}^{2}}+\frac{119}{768} \ln ^{3} \frac{\mu^{2}}{m_{h}^{2}} \\
& +n_{l}\left(\frac{3307}{15552}-\frac{1}{12} \zeta_{3}-\frac{293}{1152} \ln \frac{\mu^{2}}{m_{h}^{2}}+\frac{1}{36} \ln ^{2} \frac{\mu^{2}}{m_{h}^{2}}-\frac{1}{96} \ln ^{3} \frac{\mu^{2}}{m_{h}^{2}}\right) \\
& \left.+\xi\left(-\frac{677}{1536}+\frac{3}{32} \zeta_{3}+\frac{233}{1024} \ln \frac{\mu^{2}}{m_{h}^{2}}-\frac{3}{32} \ln ^{2} \frac{\mu^{2}}{m_{h}^{2}}+\frac{3}{256} \ln ^{3} \frac{\mu^{2}}{m_{h}^{2}}\right)\right]
\end{aligned}
$$

In Ref. [59] the Yukawa corrections to $\zeta_{m}$ and $\zeta_{g}$ enhanced by the top quark mass have been evaluated. They are conveniently expressed in terms of the variable

$$
x_{t}=\frac{G_{F} m_{t}^{2}}{8 \pi^{2} \sqrt{2}},
$$

where $m_{t}$ is the top quark mass defined in the $\overline{\mathrm{MS}}$ scheme. Corrections proportional to $x_{t}$ arise if in addition to the pure QCD Lagrangian also the couplings of the Higgs boson $(h)$ and the neutral $(\chi)$ and charged $\left(\phi^{ \pm}\right)$Goldstone boson to the top quark are considered. Sample diagrams contributing to $\zeta_{m}$ and $\zeta_{g}$ are shown in Figs. B.1 and B.2, respectively. 


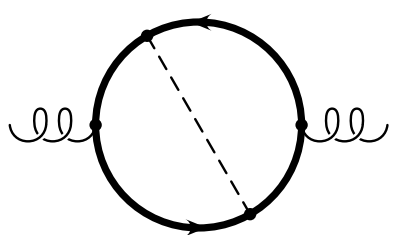

(a)

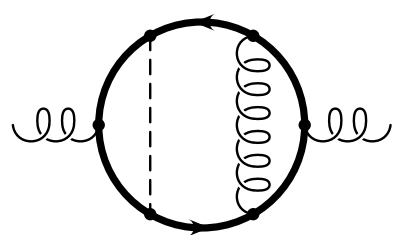

(b)

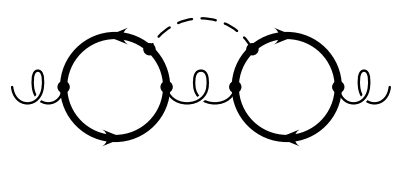

(c)

Figure B.2: Feynman diagrams contribution to $Z_{3}$ and $\zeta_{g}^{0}$. The dashed line either represents the Higgs boson $(h)$ or the neutral $(\chi)$ or charged $\left(\phi^{ \pm}\right)$Goldstone boson.

The decoupling constant for the $u, d, s$ and $c$ quark mass reads 30

$$
\begin{aligned}
\zeta_{m_{l}}^{\mathrm{MS}, x_{t}}= & +\left(\frac{\alpha_{s}^{(6)}(\mu)}{\pi}\right)^{2}\left\{\frac{89}{432}-\frac{5}{36} \ln \frac{\mu^{2}}{m_{t}^{2}}+\frac{1}{12} \ln ^{2} \frac{\mu^{2}}{m_{t}^{2}}\right. \\
& +x_{t}\left[\frac{101}{144}-\frac{5}{12} \zeta_{2}+\frac{73}{12} \zeta_{3}-9 \zeta_{4}-\frac{7}{6} \ln \frac{\mu^{2}}{m_{t}^{2}}+6 \zeta_{3} \ln \frac{\mu^{2}}{m_{t}^{2}}\right. \\
& \left.\left.+2 I_{3 l}\left(-\frac{37}{18}-\frac{19}{3} \zeta_{3}+9 \zeta_{4}-\ln \frac{\mu^{2}}{m_{t}^{2}}-6 \zeta_{3} \ln \frac{\mu^{2}}{m_{t}^{2}}\right)\right]\right\},
\end{aligned}
$$

where $I_{3 l}$ is the third component of the weak isospin, i.e. $I_{3 l}=+1 / 2$ for up-type quarks and $I_{3 l}=-1 / 2$ for down-type quark flavours. For the bottom quark one receives

$$
\begin{aligned}
\zeta_{m_{b}}^{\mathrm{MS}, x_{t}}= & \zeta_{m_{d}}+x_{t}\left\{\frac{5}{4}+\frac{3}{2} \ln \frac{\mu^{2}}{m_{t}^{2}}+\frac{\alpha_{s}^{(6)}(\mu)}{\pi}\left[\frac{16}{3}-4 \zeta_{2}+\frac{7}{2} \ln \frac{\mu^{2}}{m_{t}^{2}}+\frac{3}{2} \ln ^{2} \frac{\mu^{2}}{m_{t}^{2}}\right]\right. \\
& +\left(\frac{\alpha_{s}^{(6)}(\mu)}{\pi}\right)^{2}\left[\frac{472933}{12096}-\frac{6133}{168} \zeta_{2}+\frac{905}{72} \zeta_{3}+\frac{383}{18} \zeta_{4}+\frac{1251}{112} S_{2}+\frac{19}{72} D_{3}\right. \\
& -\frac{7}{9} B_{4}+\left(\frac{763}{18}-\frac{55}{3} \zeta_{2}-\frac{43}{4} \zeta_{3}\right) \ln \frac{\mu^{2}}{m_{t}^{2}}+\frac{529}{48} \ln ^{2} \frac{\mu^{2}}{m_{t}^{2}}+\frac{29}{12} \ln ^{3} \frac{\mu^{2}}{m_{t}^{2}} \\
& +n_{l}\left(-\frac{23}{24}+\frac{31}{36} \zeta_{2}-2 \zeta_{3}+\left(-\frac{241}{144}+\frac{2}{3} \zeta_{2}\right) \ln \frac{\mu^{2}}{m_{t}^{2}}-\frac{1}{2} \ln ^{2} \frac{\mu^{2}}{m_{t}^{2}}\right. \\
& \left.\left.\left.-\frac{1}{12} \ln ^{3} \frac{\mu^{2}}{m_{t}^{2}}\right)\right]\right\}
\end{aligned}
$$

where we have used $C_{F}=4 / 3, C_{A}=3$ and $T=1 / 2$. The constants

$$
\begin{aligned}
S_{2} & =\frac{4}{9 \sqrt{3}} \mathrm{Cl}_{2}\left(\frac{\pi}{3}\right) \approx 0.260434 \\
D_{3} & =6 \zeta_{3}-\frac{15}{4} \zeta_{4}-6\left(\mathrm{Cl}_{2}\left(\frac{\pi}{3}\right)\right)^{2} \approx-3.027009, \\
B_{4} & =16 \mathrm{Li}_{4}\left(\frac{1}{2}\right)-\frac{13}{2} \zeta_{4}-4 \zeta_{2} \ln ^{2} 2+\frac{2}{3} \ln ^{4} 2 \approx-1.762800,
\end{aligned}
$$

\footnotetext{
${ }^{30}$ For convenience, the corrections of order $\alpha_{s}^{2}$ are repeated from Eq. (B.1).
} 
where $\zeta_{4}=\pi^{4} / 90, \mathrm{Cl}_{2}$ is Clausen's function and $\mathrm{Li}_{4}$ is the quadrilogarithm, occur in the evaluation of the three-loop master diagrams [58, 207, 208, 209].

Finally, for $\zeta_{g}$ we obtain the following result:

$$
\begin{aligned}
\left(\zeta_{g}^{\mathrm{MS}, x_{t}}\right)^{2}= & +\frac{\alpha_{s}^{(6)}(\mu)}{\pi} T\left\{-\frac{1}{3} \ln \frac{\mu^{2}}{m_{t}^{2}}+\frac{\alpha_{s}^{(6)}(\mu)}{\pi}\left[C_{F}\left(-\frac{13}{48}+\frac{1}{4} \ln \frac{\mu^{2}}{m_{t}^{2}}\right)\right.\right. \\
& \left.+C_{A}\left(\frac{2}{9}-\frac{5}{12} \ln \frac{\mu^{2}}{m_{t}^{2}}\right)+T \frac{1}{9} \ln ^{2} \frac{\mu^{2}}{m_{t}^{2}}\right]+x_{t}\left\{-\frac{2}{3}+\ln \frac{\mu^{2}}{m_{t}^{2}}\right. \\
& +\frac{\alpha_{s}^{(6)}(\mu)}{\pi}\left[C_{F}\left(-\frac{17}{16}+\frac{5}{4} \zeta_{2}+\frac{25}{8} \zeta_{3}-3 \ln \frac{\mu^{2}}{m_{t}^{2}}+\frac{3}{4} \ln ^{2} \frac{\mu^{2}}{m_{t}^{2}}\right)\right. \\
& +C_{A}\left(-\frac{5}{4}+\frac{3}{8} \zeta_{2}-\frac{95}{64} \zeta_{3}+\frac{7}{4} \ln \frac{\mu^{2}}{m_{t}^{2}}\right)+T\left(\frac{5}{4}+\frac{7}{8} \zeta_{3}+\frac{4}{9} \ln \frac{\mu^{2}}{m_{t}^{2}}\right. \\
& \left.\left.\left.-\frac{2}{3} \ln ^{2} \frac{\mu^{2}}{m_{t}^{2}}\right)-\frac{7}{2} \zeta_{3} T\right\}\right\}
\end{aligned}
$$

where the contribution of the diagrams in Fig. B.2(c) corresponds to the last entry in the last line of Eq. (B.11). For convenience also the pure QCD result of $\mathcal{O}\left(\alpha_{s}^{2}\right)$ is listed. The corresponding three-loop terms can be found in [49].

In the remainder of this Appendix we want to provide the analytical results for the $x_{t}$-enhanced corrections of order $G_{F} m_{t}^{2}$ to the coefficient functions $C_{1}$ and $C_{2}$ [59]. For $C_{1}$ we have

$$
\begin{aligned}
C_{1}= & -\frac{1}{6} T \frac{\alpha_{s}^{(6)}(\mu)}{\pi}\left\{1-3 x_{t}+\frac{\alpha_{s}^{(6)}(\mu)}{\pi}\left[-C_{F} \frac{3}{4}+C_{A} \frac{5}{4}-T \frac{1}{3} \ln \frac{\mu^{2}}{m_{t}^{2}}\right.\right. \\
& \left.\left.+x_{t}\left(C_{F}\left(9-\frac{9}{2} \ln \frac{\mu^{2}}{m_{t}^{2}}\right)-C_{A} \frac{21}{4}+T\left(-\frac{2}{3}+2 \ln \frac{\mu^{2}}{m_{t}^{2}}\right)\right)\right]\right\},
\end{aligned}
$$

where $m_{t}$ is the $\overline{\mathrm{MS}}$ top quark. The $\mathcal{O}\left(\alpha_{s} x_{t}\right)$ terms can be found in 210, 75] and the $\mathcal{O}\left(\alpha_{s}^{2}\right)$ results were computed in [100, 101] The corrections of $\mathcal{O}\left(\alpha_{s} x_{t}^{2}\right)$ are taken from 59.

For the light quarks we get for $C_{2}$

$$
C_{2 l}=1+\left(\frac{\alpha_{s}^{(6)}(\mu)}{\pi}\right)^{2}\left[\frac{5}{18}-\frac{1}{3} \ln \frac{\mu^{2}}{m_{t}^{2}}+x_{t}\left(\frac{7}{3}-12 \zeta_{3}+2 I_{3 l}\left(2+12 \zeta_{3}\right)\right)\right],
$$

and in the case of the bottom quark the coefficient function reads:

$$
\begin{aligned}
C_{2 b}= & C_{2 d}+x_{t}\left\{-3+\frac{\alpha_{s}^{(6)}(\mu)}{\pi}\left[-7-6 \ln \frac{\mu^{2}}{m_{t}^{2}}\right]+\left(\frac{\alpha_{s}^{(6)}(\mu)}{\pi}\right)^{2}\left[-\frac{12169}{144}+\frac{110}{3} \zeta_{2}\right.\right. \\
& +\frac{43}{2} \zeta_{3}-\frac{89}{2} \ln \frac{\mu^{2}}{m_{t}^{2}}-\frac{55}{4} \ln ^{2} \frac{\mu^{2}}{m_{t}^{2}}+n_{l}\left(\frac{241}{72}-\frac{4}{3} \zeta_{2}+2 \ln \frac{\mu^{2}}{m_{t}^{2}}\right. \\
& \left.\left.\left.+\frac{1}{2} \ln ^{2} \frac{\mu^{2}}{m_{t}^{2}}\right)\right]\right\} .
\end{aligned}
$$

In [75] $C_{2 l}$ and $C_{2 b}$ are listed for general gauge group $S U\left(N_{c}\right)$. 


\section{Analytical results for $R(s)$}

As in the literature the quadratic and quartic correction terms to $R(s)$ are not yet available in analytic form we want to provide the corresponding results using the notation introduced in Eqs. (4.73) and (4.74). For completeness also the massless approximation is given. Of course, it is the same in the $\overline{\mathrm{MS}}$ and on-shell schemes

$$
\begin{aligned}
r_{0}= & +\frac{\alpha_{s}}{\pi}+\left(\frac{\alpha_{s}}{\pi}\right)^{2}\left[\frac{365}{24}-11 \zeta_{3}+n_{f}\left(-\frac{11}{12}+\frac{2}{3} \zeta_{3}\right)\right] \\
+ & \left(\frac{\alpha_{s}}{\pi}\right)^{3}\left[\frac{87029}{288}-\frac{121}{8} \zeta_{2}-\frac{1103}{4} \zeta_{3}+\frac{275}{6} \zeta_{5}+n_{f}\left(-\frac{7847}{216}+\frac{11}{6} \zeta_{2}\right.\right. \\
+ & \left.\left.\frac{262}{9} \zeta_{3}-\frac{25}{9} \zeta_{5}\right)+n_{f}^{2}\left(\frac{151}{162}-\frac{1}{18} \zeta_{2}-\frac{19}{27} \zeta_{3}\right)\right], \\
r_{Q, 2}^{\mathrm{OS}}= & \frac{M_{Q}^{2}}{s} \frac{\alpha_{s}}{\pi}\left[12+\frac{\alpha_{s}}{\pi}\left(\frac{189}{2}+24 L_{m s}-\frac{13}{3} n_{f}\right)+\left(\frac{\alpha_{s}}{\pi}\right)^{2}\left(\frac{22351}{12}-\frac{967}{2} \zeta_{2}\right.\right. \\
& -16 \zeta_{2} \ln 2+\frac{502}{3} \zeta_{3}-\frac{5225}{6} \zeta_{5}+378 L_{m s}-9 L_{m s}^{2}+n_{f}\left(-\frac{8429}{54}+42 \zeta_{2}\right. \\
& \left.\left.\left.-\frac{466}{27} \zeta_{3}+\frac{1045}{27} \zeta_{5}-\frac{52}{3} L_{m s}+2 L_{m s}^{2}\right)+n_{f}^{2}\left(\frac{125}{54}-\frac{2}{3} \zeta_{2}\right)\right)\right], \\
r_{q Q, 2}^{\mathrm{OS}}= & \frac{M_{Q}^{2}}{s}\left(\frac{\alpha_{s}}{\pi}\right)^{3}\left[-80+60 \zeta_{3}+n_{f}\left(\frac{32}{9}-\frac{8}{3} \zeta_{3}\right)\right],
\end{aligned}
$$

where $L_{m s}=\ln M_{Q}^{2} / s$.

$$
\begin{aligned}
r_{Q, 4}^{\mathrm{OS}}= & \left(\frac{M_{Q}^{2}}{s}\right)^{2}\left[-6+\frac{\alpha_{s}}{\pi}\left(10-24 L_{m s}\right)+\left(\frac{\alpha_{s}}{\pi}\right)^{2}\left(\frac{206}{3}+218 \zeta_{2}+16 \zeta_{2} \ln 2+104 \zeta_{3}\right.\right. \\
& \left.-\frac{311}{2} L_{m s}-15 L_{m s}^{2}+n_{f}\left(-\frac{35}{9}-12 \zeta_{2}-\frac{8}{3} \zeta_{3}+9 L_{m s}-2 L_{m s}^{2}\right)\right) \\
& +\left(\frac{\alpha_{s}}{\pi}\right)^{3}\left(\frac{91015}{108}-\frac{76}{9} \ln ^{4} 2+\frac{2564287}{540} \zeta_{2}-\frac{4568}{9} \zeta_{2} \ln 2-\frac{128}{3} \zeta_{2} \ln ^{2} 2\right. \\
& +\frac{56257}{18} \zeta_{3}-\frac{1439}{3} \zeta_{2} \zeta_{3}-\frac{1565}{6} \zeta_{4}-\frac{3770}{3} \zeta_{5}-\frac{608}{3} a_{4}+L_{m s}\left(-\frac{5536}{3}+564 \zeta_{2}\right. \\
& \left.-24 \zeta_{2} \ln 2+416 \zeta_{3}\right)-\frac{591}{4} L_{m s}^{2}+\frac{15}{2} L_{m s}^{3}+n_{f}\left(-\frac{21011}{216}+\frac{8}{27} \ln ^{4} 2-\frac{3544}{9} \zeta_{2}\right. \\
& -\frac{176}{9} \zeta_{2} \ln 2+\frac{32}{9} \zeta_{2} \ln ^{2} 2-\frac{2323}{9} \zeta_{3}+\frac{700}{9} \zeta_{4}+\frac{440}{9} \zeta_{5}+\frac{64}{9} a_{4}+L_{m s}\left(\frac{2419}{12}\right. \\
& \left.\left.+\frac{44}{3} \zeta_{2}+\frac{16}{3} \zeta_{2} \ln 2+\frac{28}{3} \zeta_{3}\right)-\frac{157}{6} L_{m s}^{2}-\frac{2}{3} L_{m s}^{3}\right)+n_{f}^{2}\left(\frac{35}{18}+\frac{25}{3} \zeta_{2}+\frac{112}{27} \zeta_{3}\right. \\
& \left.\left.\left.+L_{m s}\left(-\frac{94}{27}-\frac{8}{3} \zeta_{2}\right)+\frac{13}{9} L_{m s}^{2}-\frac{2}{9} L_{m s}^{3}\right)\right)\right], \\
r_{q Q, 4}^{\mathrm{OS}}= & \left(\frac{M_{Q}^{2}}{s}\right)^{2}\left(\frac{\alpha_{s}}{\pi}\right)^{2}\left[\frac{13}{3}-4 \zeta_{3}-L_{m s}+\frac{\alpha_{s}}{\pi}\left(-\frac{4217}{48}+15 \zeta_{2}+\frac{139}{3} \zeta_{3}+\frac{50}{3} \zeta_{5}\right.\right.
\end{aligned}
$$




$$
\begin{aligned}
& +L_{m s}\left(\frac{97}{4}-38 \zeta_{3}\right)-2 L_{m s}^{2}+n_{f}\left(\frac{457}{108}-\frac{2}{3} \zeta_{2}-\frac{22}{9} \zeta_{3}\right. \\
& \left.\left.\left.+L_{m s}\left(-\frac{13}{18}+\frac{4}{3} \zeta_{3}\right)\right)\right)\right]
\end{aligned}
$$

with $a_{4}=\operatorname{Li}_{4}(1 / 2) \approx 0.517479$.

\section{Note added:}

In the meantime a third independent evaluation of the order $\alpha^{2}$ QED corrections to the muon decay became available [211].

\section{References}

[1] G. 't Hooft and M. Veltman, Nucl. Phys. B 44 (1972) 189.

[2] G. 't Hooft and M. Veltman, Nucl. Phys. B 50 (1972) 318.

[3] G. 't Hooft and M. Veltman, Nucl. Phys. B 153 (1979) 365.

[4] C.G. Bollini and J.J. Giambiagi, Phys. Lett. B 40 (1972) 566; Nuovo Cim. B 12 (1972) 20.

[5] R. Gastmans and R. Meuldermans, Nucl. Phys. B 63 (1973) 277;

W. J. Marciano and A. Sirlin, Nucl. Phys. B 88, 86 (1975);

W. J. Marciano, Phys. Rev. D 12, 3861 (1975).

[6] A. Sirlin, Phys. Rev. D 22 (1980) 971.

[7] G. Passarino and M. Veltman, Nucl. Phys. B 160 (1979) 151.

[8] W. F. Hollik, Fortsch. Phys. 38 (1990) 165.

[9] A. Denner, Fortsch. Phys. 41 (1993) 307.

[10] G. Weiglein, R. Scharf, and M. Böhm, Nucl. Phys. B 416 (1994) 606;

S. Bauberger, F.A. Berends, M. Böhm and M. Buza, Nucl. Phys. B 434 (1995) 383.

[11] T. Gehrmann and E. Remiddi, Report No.: hep-ph/0101147; and references therein.

[12] G. 't Hooft, Nucl. Phys. B 61 (1973) 455.

[13] W.A. Bardeen, A.J. Buras, D.W. Duke, and T. Muta, Phys. Rev. D 18 (1978) 3998.

[14] T. van Ritbergen, J.A.M. Vermaseren, and S.A. Larin Phys. Lett. B 400 (1997) 379.

[15] K.G. Chetyrkin, Phys. Lett. B 404 (1997) 161. 
[16] S.A. Larin, T. van Ritbergen, and J.A.M. Vermaseren, Phys. Lett. B 405 (1997) 327.

[17] T. Kinoshita and A. Sirlin, Phys. Rev. 113 (1959) 1652;

S.M. Berman, Phys. Rev. 112 (1958) 267.

[18] T. van Ritbergen and R. Stuart, Phys. Rev. Lett. 82 (1999) 488; Nucl. Phys. B $564(2000) 343$.

[19] T. Seidensticker and M. Steinhauser, Phys. Lett. B 467 (1999) 271.

[20] N.N. Bogoliubov and O.S. Parasiuk, Acta. Math. 97 (1957) 227;

O.S. Parasiuk, Ukr. Math. Z. 12 (1960) 287.

[21] K. Hepp, Comm. Math. Phys. 2 (1966) 301.

[22] J.C. Collins, Renormalization (Cambridge University Press, 1995).

[23] T. Muta, Foundations of Quantum Chromodynamics (World Scientific, Singapore, 1987).

[24] K.G. Chetyrkin, Report No.: MPI-Ph/PTh 13/91.

[25] J.C. Collins, Nucl. Phys. B 92 (1975) 477.

[26] A.A. Vladimirov, Teor. Mat. Fiz. 43 (1980) 210.

[27] K.G. Chetyrkin, A.L. Kataev, and F.V. Tkachov, Nucl. Phys. B 174 (1980) 345.

[28] W.A. Caswell and A.D. Kennedy, Phys. Rev. D 25 (1982) 392.

[29] K.G. Chetyrkin and F.V. Tkachov, Phys. Lett. B 114 (82) 340.

[30] K.G. Chetyrkin and V.A. Smirnov, Phys. Lett. B 144 (84) 419.

[31] S.A. Larin, F.V. Tkachov, and J.A.M. Vermaseren, Rep. No. NIKHEF-H/91-18 (Amsterdam, 1991).

[32] R. Tarrach, Nucl. Phys. B 183 (1981) 384.

[33] O.V. Tarasov, Report No.: preprint JINR P2-82-900 (1982).

[34] S.A. Larin, Report Nos.: Preprint NIKHEF-H/92-18 (1992), hep-ph/9302240; In Proc. of the Int. Baksan School "Particles and Cosmology" (April 22-27, 1993, Kabardino-Balkaria, Russia), eds. E.N. Alexeev, V.A. Matveev, Kh.S. Nirov, V.A. Rubakov (World Scientific, Singapore, 1994).

[35] M. Steinhauser, Comp. Phys. Commun. 134 (2001) 335.

[36] K.G. Chetyrkin, Acta Phys. Polon. B 28 (1997) 725. 
[37] K.G. Chetyrkin, private communication and in preparation.

[38] K.G. Chetyrkin, Phys. Lett. B 391 (1997) 402.

[39] L.R. Surguladze and M.A. Samuel, Phys. Rev. Lett. 66 (1991) 560; (E) ibid., 2416;

S.G. Gorishny, A.L. Kataev, and S.A. Larin, Phys. Lett. B 259 (1991) 144.

[40] K.G. Chetyrkin, Phys. Lett. B 390 (1997) 309.

[41] K.G. Chetyrkin and M. Steinhauser, unpublished;

M. Steinhauser, published in the proceedings of $4^{\text {th }}$ International Symposium on Radiative Corrections (RADCOR 98): Applications of Quantum Field Theory to Phenomenology, Barcelona, Catalonia, Spain, 8-12 Sep 1998. Report No.: hepph/9811342.

[42] S. Wolfram, Mathematica - a system for doing mathematics by computer (AddisonWesley, New York, 1988).

[43] K.G. Chetyrkin, M. Misiak, and M. Münz, Nucl. Phys. B 518 (1998) 473.

[44] D.J. Gross and F. Wilczek, Phys. Rev. Lett. 30 (1973) 1343; Phys. Rev. D 8 (1973) 3633 ;

H.D. Politzer, Phys. Rev. Lett. 30 (1973) 1346.

[45] D.R.T. Jones, Nucl. Phys. B 75 (1974) 531;

W.E. Caswell, Phys. Rev. Lett. 33 (1974) 244;

É.Sh. Egoryan and O.V. Tarasov, Teor. Mat. Fiz. 41 (1979) 26; Theor. Math. Phys. 41 (1979) 863.

[46] O.V. Tarasov, A.A. Vladimirov, and A.Yu. Zharkov, Phys. Lett. B 93 (1980) 429;

S.A. Larin and J.A.M. Vermaseren, Phys. Lett. B 303 (1993) 334.

[47] F.V. Tkachov, Phys. Lett. B 100 (1981) 65;

K.G. Chetyrkin and F.V. Tkachov, Nucl. Phys. B 192 (1981) 159.

[48] T. Appelquist and J. Carazzone, Phys. Rev. D 11 (1975) 2856.

[49] K.G. Chetyrkin, B.A. Kniehl, and M. Steinhauser, Nucl. Phys. B 510 (1998) 61.

[50] S. Weinberg, Phys. Lett. B 91 (1980) 51;

B.A. Ovrut and H.J. Schnitzer, Phys. Lett. B 100 (1981) 403.

[51] W. Wetzel, Nucl. Phys. B 196 (1982) 259;

W. Bernreuther and W. Wetzel, Nucl. Phys. B 197 (1982) 228; (E) ibid. 513 (1998) 758 ;

W. Bernreuther, Ann. Phys. 151 (1983) 127; Z. Phys. C 20 (1983) 331. 
[52] S.A. Larin, T. van Ritbergen, and J.A.M. Vermaseren, Nucl. Phys. B 438 (1995) 278.

[53] W. Bernreuther, private communication; see also footnote 2 in Ref. [89].

[54] C. Becchi, A. Rouet, and R. Stora, Comm. Math. Phys. 42 (1975) 127; Ann. Phys. B 98 (1976) 287;

I.V. Tyutin, Lebeev Institute preprint N39 (1975).

[55] J.A.M. Vermaseren, Symbolic Manipulation with FORM, (Computer Algebra Netherlands, Amsterdam, 1991).

[56] S.G. Gorishny and S.A. Larin, Nucl. Phys. B 283 (1987) 452.

[57] P. Nogueira, J. Comp. Phys. 105 (1993) 279.

[58] D.J. Broadhurst, Z. Phys. C 54 (1992) 599.

[59] M. Steinhauser, Phys. Rev. D 49 (1999) 054005.

[60] K.G. Chetyrkin, A.L. Kataev, and F.V. Tkachov, Phys. Lett. B 85 (1979) 277;

M. Dine and J. Sapirstein, Phys. Rev. Lett. 43 (1979) 668;

W. Celmaster and R.J. Gonsalves, Phys. Rev. Lett. 44 (1980) 560.

[61] A.L. Kataev and V.V. Starshenko, Mod. Phys. Lett. A 10 (1995) 235; Phys. Rev. D 52 (1995) 402;

M.A. Samuel, J. Ellis, and M. Karliner, Phys. Rev. Lett. 74 (1995) 4380;

P.A. Rączka and A. Szymacha, Z. Phys. C 70 (1996) 125; Phys. Rev. D 54 (1996) 3073;

J. Ellis, E. Gardi, M. Karliner, and M.A. Samuel, Phys. Lett. B 366 (1996) 268; Phys. Rev. D 54 (1996) 6986;

S. Groote, J.G. Körner, A.A. Pivovarov, and K. Schilcher, Phys. Rev. Lett. 79 (1997) 2763;

S. Groote, J.G. Körner, and A.A. Pivovarov, Phys. Lett. B 407 (1997) 66.

[62] K.G. Chetyrkin, B.A. Kniehl, and A. Sirlin, Phys. Lett. B 402 (1997) 359.

[63] G. Rodrigo and A. Santamaria, Phys. Lett. B 313 (1993) 441.

[64] K.G. Chetyrkin, J.H. Kühn, and M. Steinhauser, Comp. Phys. Commun. 133 (2000) 43.

[65] G.T. Bodwin, E. Braaten, and G.P. Lepage, Phys. Rev. D 51 (1995) 1125; (E) ibid. 55 (1997) 5853.

[66] N. Brambilla, Report No.: hep-ph/0012026.

[67] A.A. Penin, Nucl. Phys. Proc. Suppl. 96 (2001) 418. 
[68] K.G. Wilson, Phys. Reports 179 (1969) 1499.

[69] H. Kluberg-Stern and J.B. Zuber, Phys. Rev. D 12 (1975) 467.

[70] N.K. Nielsen, Nucl. Phys. B 97 (1975) 527; Nucl. Phys. B 120 (1977) 212.

[71] V.P. Spiridonov, Rep. No. INR P-0378 (Moscow, 1984).

[72] K.G. Chetyrkin and V.P. Spiridonov, Yad. Fiz. 47 (1988) 818 (Sov. J. Nucl. Phys. 47 (1988) 522).

[73] K.G. Chetyrkin and J.H. Kühn, Nucl. Phys. B 432 (1994) 337.

[74] R. Harlander, Ph. D. thesis, University of Karlsruhe (Shaker Verlag, Aachen, 1998).

[75] K.G. Chetyrkin, B.A. Kniehl, and M. Steinhauser, Phys. Rev. Lett. 78 (1997) 594; Nucl. Phys. B 490 (1997) 19.

[76] B.A. Kniehl and A. Sirlin, Phys. Lett. B 318 (1993) 367;

B.A. Kniehl, Phys. Rev. D 50 (1994) 3314;

A. Djouadi and P. Gambino, Phys. Rev. D 51 (1995) 218.

[77] B.A. Kniehl and M. Steinhauser, Nucl. Phys. B 454 (1995) 485; Phys. Lett. B 365 (1996) 297.

[78] K.G. Chetyrkin, B.A. Kniehl, M. Steinhauser, and W.A. Bardeen, Nucl. Phys. B 535 (1998) 3.

[79] K.G. Chetyrkin, B.A. Kniehl, and M. Steinhauser, Phys. Rev. Lett. 79 (1997) 2184.

[80] R.V. Harlander, Phys. Lett. B 492 (2000) 74.

[81] R.V. Harlander and W.B. Kilgore, Phys. Rev. D 64 (2001) 013015.

[82] S. Catani, D. de Florian and M. Grazzini, JHEP 0105 (2001) 025.

[83] B.S. DeWitt, Phys. Rev. 162 (1967) 1195;

G. 't Hooft, in Karpacz 1975, Proceedings, Acta Universitatis Wratislaviensis No.368, Vol.1, Wroclaw 1976, 345-369;

H. Kluberg-Stern and J.B. Zuber, Phys. Rev. D 12 (1975) 482; Phys. Rev. D 12 (1975) 3159;

S. Ichinose and M. Omote, Nucl. Phys. B 203 (1982) 221;

D.M. Capper and A. MacLean, Nucl. Phys. B 203 (1982) 413;

D.G. Boulware, Phys. Rev. D 23 (1981) 389.

[84] L.F. Abbott, Nucl. Phys. B 185 (1981) 189.

[85] A. Denner, G. Weiglein, and S. Dittmaier, Phys. Lett. B 333 (1994) 420; Nucl. Phys. B 440 (1995) 95. 
[86] P.A. Grassi, Nucl. Phys. B 462 (1996) 524; Nucl. Phys. B 537 (1999) 527; Nucl. Phys. B 560 (1999) 499.

[87] B.A. Kniehl and M. Spira, Z. Phys. C 69 (1995) 77.

[88] B.A. Kniehl, Phys. Rept. 240 (1994) 211.

[89] M. Spira, Fortsch. Phys. 46 (1998) 203.

[90] E. Braaten and J.P. Leveille, Phys. Rev. D 22 (1980) 715.

[91] M. Drees and K. Hikasa, Phys. Lett. B 240 (1990) 455. (E) ibid. B 262 (1991) 497.

[92] S.G. Gorishny, A.L. Kataev, S.A. Larin, and L.R. Surguladze Mod. Phys. Lett. A 5 (1990) 2703; Phys. Rev. D 43 (1991) 1633.

[93] L.R. Surguladze, Phys. Lett. B 341 (1994) 60.

[94] K.G. Chetyrkin and A. Kwiatkowski, Nucl. Phys. B 461 (1996) 3.

[95] R. Harlander and M. Steinhauser, Phys. Rev. D 56 (1997) 3980.

[96] A.L. Kataev and V.T. Kim, Report No.: hep-ph/9304282.

[97] A.L. Kataev, JETP Lett. 66 (1997) 327.

[98] S.A. Larin, T. van Ritbergen, and J.A.M. Vermaseren, Phys. Lett. B 362 (1995) 134.

[99] K.G. Chetyrkin and M. Steinhauser, Phys. Lett. B 408 (1997) 320.

[100] T. Inami, T. Kubota, and Y. Okada, Z. Phys. C 18 (1983) 69.

[101] A. Djouadi, M. Spira, and P.M. Zerwas, Phys. Lett. B 264 (1991) 440;

M. Spira, A. Djouadi, D. Graudenz, and P.M. Zerwas, Nucl. Phys. B 453 (1995) 17.

[102] B.A. Kniehl, Phys. Lett. B 343 (1995) 299.

[103] A. Dabelstein and W. Hollik, Z. Phys. C 53 (1992) 507.

[104] B.A. Kniehl, Nucl. Phys. B 376 (1992) 3.

[105] A. Kwiatkowski and M. Steinhauser, Phys. Lett. B 338 (1994) 66; B 342 (1995) $455(\mathrm{E})$;

B.A. Kniehl and M. Spira, Nucl. Phys. B 432 (1994) 39.

[106] K.G. Chetyrkin, J.H. Kühn, and A. Kwiatkowski, Phys. Reports 277 (1996) 189.

[107] K.G. Chetyrkin, J.H. Kühn, and A. Kwiatkowski, Phys. Reports 277 (1997) 189. 
[108] K.G. Chetyrkin and J.H. Kühn, Phys. Lett. B 342 (1995) 356.

[109] K.G. Chetyrkin, J.H. Kühn, and T. Teubner, Phys. Rev. D 56 (1997) 3011.

[110] R. Harlander and M. Steinhauser, Eur. Phys. J. C 2 (1998) 151.

[111] G. Källen and A. Sabry, K. Dan. Videnk. Selsk. Mat.-Fys. Medd. 29 (1955) No. 17; see also: J. Schwinger, Particles, Sources and Fields, Vol. II, (Addison-Wesley, New York, 1973).

[112] A.H. Hoang, J.H. Kühn, and T. Teubner, Nucl. Phys. B 452 (1995) 173.

[113] K.G. Chetyrkin and J.H. Kühn, Phys. Lett. B 248 (1990) 359.

[114] S.G. Gorishny, A.L. Kataev, and S.A. Larin, Nuovo Cim. A 92 (1986) 119.

[115] R. Harlander, Acta Phys. Polon. B 30 (1999) 3443.

[116] R. Harlander and M. Steinhauser, Prog. Part. Nucl. Phys. 43 (1999) 167.

[117] S.G. Gorishny, S.A. Larin, and F.V. Tkachov, Phys. Lett. B 124 (1983) 217.

[118] L.R. Surguladze and F.V. Tkachov, Nucl. Phys. B 331 (1990) 35.

[119] D.J. Broadhurst, Phys. Lett. B 101 (1981) 423.

[120] E. Braaten, S. Narison, and A. Pich, Nucl. Phys. B 373 (1992) 581.

[121] K.G. Chetyrkin, R.V. Harlander, and J.H. Kühn, Nucl. Phys. B 586 (2000) 56, and private communication.

[122] K.G. Chetyrkin and J.H. Kühn, Phys. Lett. B 406 (1997) 102.

[123] N. Gray, D.J. Broadhurst, W. Grafe, and K. Schilcher, Z. Phys. C 48 (1990) 673.

[124] K.G. Chetyrkin and M. Steinhauser, Phys. Rev. Lett. 83 (1999) 4001.

[125] K.G. Chetyrkin and M. Steinhauser, Nucl. Phys. B 573 (2000) 617.

[126] K. Melnikov and T. van Ritbergen, Phys. Lett. B 482 (2000) 99.

[127] K.G. Chetyrkin, A.H. Hoang, J.H. Kühn, M. Steinhauser, and T. Teubner, Eur. Phys. J. C 2 (1998) 137.

[128] See, e.g.,

J. Ellis, I. Jack, D.R. Jones, M. Karliner, and M.A. Samuel, Phys. Rev. D 57 (1998) 2665 ;

V. Elias, F.A. Chishtie, and T.G. Steele, J. Phys. G 26 (2000) 1239. 
[129] V.A. Smirnov, Renormalization and Asymptotic Expansion (Birkhäuser, Basel, 1991);

V.A. Smirnov, Mod. Phys. Lett. A 10 (1995) 1485 ;

and references therein.

[130] J. Fleischer and O.V. Tarasov, Z. Phys. C 64 (1994) 413

[131] K.G. Chetyrkin, R. Harlander, and M. Steinhauser, Phys. Rev. D 58 (1998) 014012.

[132] P.A. Baikov and D.J. Broadhurst, 4th International Workshop on Software Engineering and Artificial Intelligence for High Energy and Nuclear Physics (AIHENP95), Pisa, Italy, 3-8 April 1995. Published in Pisa AIHENP (1995) 167.

[133] R.V. Harlander, Report No.: hep-ph/0102266.

[134] K.G. Chetyrkin, J.H. Kühn, and M. Steinhauser, Phys. Lett. B 371 (1996) 93; Nucl. Phys. B 482 (1996) 213.

[135] K.G. Chetyrkin, J.H. Kühn, and M. Steinhauser, Nucl. Phys. B 505 (1997) 40.

[136] K.G. Chetyrkin, R. Harlander, J.H. Kühn, and M. Steinhauser, Nucl. Inst. Meth. A 389 (1997) 354.

[137] K.G. Chetyrkin, R. Harlander, J.H. Kühn, and M. Steinhauser, Nucl. Phys. B 503 (1997) 339.

[138] K.G. Chetyrkin, A.H. Hoang, J.H. Kühn, M. Steinhauser, and T. Teubner, Phys. Lett. B 384 (1996) 233.

[139] J.H. Kühn, T. Hahn, and R. Harlander, Report Nos.: TTP99-50,KA-TP-26-1999, BNL-HET-99/42 and hep-ph/9912262.

[140] W.H. Furry, Phys. Rev. 51 (1937) 125.

[141] L.D. Landau, Docl. Akad. Nauk USSR 60 (1948) 207;

C.N. Yang, Phys. Rev. 77 (1950) 242.

[142] B.A. Kniehl and J.H. Kühn, Phys. Lett. B 224 (1989) 229; Nucl. Phys. B 329 (1990) 547.

[143] S. Eidelman and F. Jegerlehner, Z. Phys. C 67 (1995) 585.

[144] M. Davier and A. Höcker, Phys. Lett. B 419 (1998) 419.

[145] J.H. Kühn and M. Steinhauser, Phys. Lett. B 437 (1998) 425.

[146] S. Groote, J.G. Körner, K. Schilcher, and N.F. Nasrallah, Phys. Lett. B 440 (1998) 375. 
[147] J. Erler, Phys. Rev. D 59 (1999) 054008.

[148] M. Davier and A. Höcker, Phys. Lett. B 435 (1998) 427.

[149] F. Jegerlehner, Proceedings of the IVth International Symposium on Radiative Corrections (RADCOR 98): Applications of Quantum Field Theory to Phenomenology, Barcelona, Catalonia, Spain, Sept. 8-12, 1998, Report No.: hep-ph/9901386.

[150] A.D. Martin, J. Outhwaite, and M.G. Ryskin, Eur. Phys. J. C 19 (2001) 681.

[151] M. Steinhauser, Phys. Lett. B 429 (158) 1998.

[152] A.D. Martin and D. Zeppenfeld, Phys. Lett. B 345 (1995) 558.

[153] H. Burkhardt and B. Pietrzyk, Phys. Lett. B 356 (1995) 389.

[154] M.L. Swartz, Phys. Rev. D 53 (1996) 5268.

[155] R. Alemany, M. Davier, and A. Höcker, Eur. Phys. J. C 2 (1998) 123.

[156] H. Burkhardt and B. Pietrzyk, Report No.: LAPP-EXP 2001-03.

[157] F. Jegerlehner, Report No.: hep-ph/0105283.

[158] B.A. Kniehl, G. Kramer, and B. Pötter, Phys. Rev. Lett. 85 (2000) 5288.

[159] J. Burmester et al. (PLUTO Coll.), Phys. Lett. B 66 (1977) 395;

L. Criegee and G. Knies, Phys. Reports 83 (1982) 151.

[160] R. Brandelik et al. (DASP Coll.), Phys. Lett. B 76 (1978) 361;

A. Petersen, Ph.D. thesis, University of Hamburg, (1978).

[161] J.L. Siegrist et al. (MARK I Coll.), Phys. Rev. D 26 (1982) 969.

[162] J.Z. Bai et al. [BES Collaboration], Phys. Rev. Lett. 84 (2000) 594; hep-ex/0102003.

[163] K.G. Chetyrkin and M. Steinhauser, Phys. Lett. B 502 (2001) 104.

[164] K.G. Chetyrkin and M. Steinhauser, Eur. Phys. J. C 21 (2001) 319.

[165] See, e.g.,

A.G. Grozin, Lectures on perturbative HQET 1, Report No.: hep-ph/0008300;

A.V. Manohar and M.B. Wise, Heavy quark physics, Cambridge University Press (2000).

[166] X. Ji and M.J. Musolf, Phys. Lett. B 257 (1991) 409;

D.J. Broadhurst and A.G. Grozin, Phys. Lett. B 267 (1991) 105;

V. Giménez, Nucl. Phys. B 375 (1992) 582.

[167] D.J. Broadhurst and A.G. Grozin, Phys. Rev. D 52 (1995) 4082. 
[168] A.G. Grozin, Phys. Lett. B 445 (1998) 165.

[169] D.J. Broadhurst and A.G. Grozin, Phys. Lett. B 274 (1992) 421.

[170] A. Czarnecki and K. Melnikov, Report No.: hep-ph/0110028.

[171] M.C. Smith and S. Willenbrock, Phys. Rev. D D54 (1996) 6696.

[172] R. Hamberg, W. van Neerven, and T. Matsuura, Nucl. Phys. B 359 (1991) 343;

W. van Neerven and E. Zijlstra, Nucl. Phys. B 382 (1992) 11.

[173] A.D. Martin, R.G. Roberts, W.J. Stirling, and R.S. Thorne, Eur. Phys. J. C 18 (2000) 117.

[174] D.E. Groom et al., Eur. Phys. J. C 15 (2000) 1.

[175] A. Ferroglia, G. Ossola, and A. Sirlin, Nucl. Phys. B 560 (1999) 23.

[176] S.M. Berman and A. Sirlin, Ann. Phys. 20 (1962) 20.

[177] K.G. Chetyrkin, R. Harlander, T. Seidensticker, and M. Steinhauser, Phys. Rev. D 60 (1990) 114015.

[178] T. van Ritbergen and R. Stuart, Phys. Lett. B 437 (1998) 201.

[179] J. Fleischer, V.A. Smirnov, and O.V. Tarasov, Z. Phys. C 74 (1997) 379.

[180] T. Seidensticker, Diploma thesis (University of Karlsruhe, 1998), unpublished.

[181] T. van Ritbergen, Phys. Lett. B 454 (1999) 353.

[182] K. Melnikov and T. van Ritbergen, Nucl. Phys. B 591 (2000) 515.

[183] S. Laporta and E. Remiddi, Phys. Lett. B 379 (1996) 283.

[184] A.S. Kronfeld, Phys. Rev. D 58 (1998) 051501.

[185] P. Gambino and P.A. Grassi, Phys. Rev. D 62 (2000) 076002.

[186] J. Fleischer, F. Jegerlehner, O.V. Tarasov, and O.L. Veretin, Nucl. Phys. B 539 (1999) 671, (E) ibid. B 571 (2000) 511.

[187] A.H. Hoang, Report No.: hep-ph/0008102.

[188] G. Grunberg, Phys. Lett. B 95 (1980) 70, Phys. Lett. B 110 (1982) 501, Phys. Rev. D 29 (1984) 2315.

[189] P.M. Stevenson, Phys. Rev. D 23 (1981) 1916, Phys. Lett. B 100 (1981) 61, Nucl. Phys. B 203 (1982) 472, Phys. Lett. B 231 (1984) 65. 
[190] M. Beneke and V.M. Braun, Phys. Lett. B 348 (1995) 513.

[191] M. Beneke and V.M. Braun, Nucl. Phys. B 426 (1994) 301.

[192] I.I. Bigi, M.A. Shifman, N.G. Uraltsev, and A.I. Vainshtein, Phys. Rev. D 50 (1994) 2234

[193] M. Beneke, Phys. Lett. B 434 (1998) 115.

[194] A.H. Hoang, M.C. Smith, T. Stelzer, and S. Willenbrock, Phys. Rev. D 59 (1999) 114014.

[195] A.H. Hoang and T. Teubner, Phys. Rev. D 60 (1999) 114027.

[196] V.A. Smirnov, Phys. Lett. B 394 (1997) 205;

A. Czarnecki and V. A. Smirnov, Phys. Lett. B 394 (1997) 211.

[197] V.A. Smirnov, Phys. Lett. B 404 (1997) 101.

[198] M. Beneke and V.A. Smirnov, Nucl. Phys. B 522 (1998) 321.

[199] K.G. Chetyrkin, Phys. Lett. B 307 (1993) 169.

[200] A.H. Hoang, M. Jeżabek, J.H. Kühn, and T. Teubner, Phys. Lett. B 338 (1994) 330.

[201] T. Teubner, Ph. D. thesis, University of Karlsruhe (Shaker Verlag, Aachen, 1996).

[202] M. Steinhauser, Ph. D. thesis, University of Karlsruhe (Shaker Verlag, Aachen, 1996).

[203] V.A. Smirnov, Phys. Lett. B 465 (1999) 226.

[204] G.J. van Oldenborgh and J.A. Vermaseren, Z. Phys. C 46 (1990) 425.

[205] G. Weiglein, Report No.: hep-ph/0109237;

and references therein.

[206] R. Harlander, T. Seidensticker, and M. Steinhauser, Phys. Lett. B 426 (1998) 125.

[207] L. Avdeev, J. Fleischer, S. Mikhailov, and O. Tarasov, Phys. Lett. B 336 (1994) 560; (E) ibid. B 349 (1995) 597.

[208] K.G. Chetyrkin, J.H. Kühn, and M. Steinhauser, Phys. Lett. B 351 (1995) 331.

[209] D.J. Broadhurst, Eur. Phys. J. C 8 (1999) 311.

[210] A. Djouadi and P. Gambino, Phys. Rev. Lett. 73 (1994) 2528.

[211] A. Czarnecki and K. Melnikov, Report No.: hep-ph/0112264. 